\title{
Molecular Structure and
}

Functiond Activity of Nerve Cells

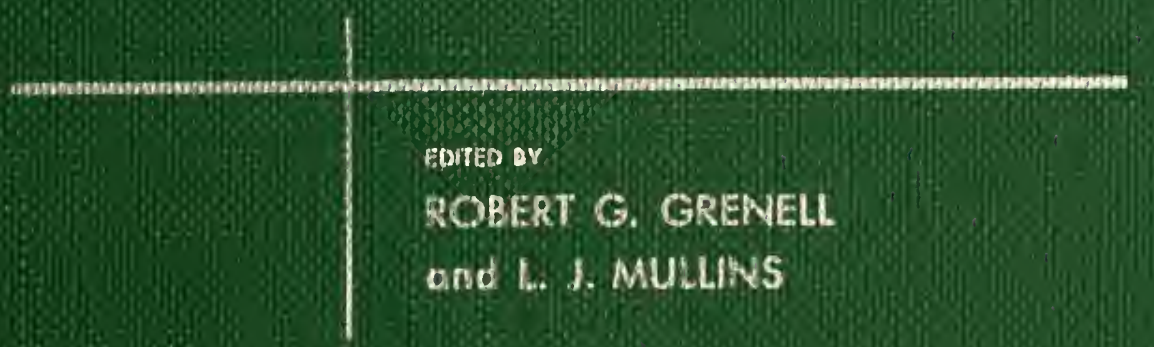

A.IB.S.

QH

611

A 62 



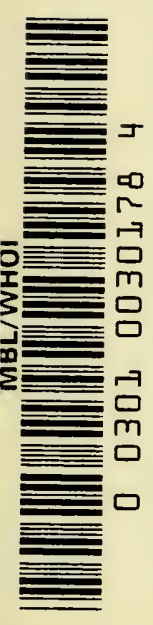



Molecular Structure and

Functional Activity of Nerve Cells 



\section{Molecular Structure and \\ Functional Activity of Nerve Cells}

A Symposium organized by the American

Institute of Biological Sciences and sponsored by the Office of Naval Research. Held in Washington, D.C., June 3-4, 1955.

EDITED BI

ROBERT G. GRENELL

AND

L. J. MULLINS

PUBLICATION

No. 1

1956

AMERICAN INSTITUTE OF BIOLOGICAL SCIENCES WASHINGTON, D. C. 
${ }^{(ㄷ)} 1956, \mathrm{BY}$

American Institute of Biological Sciences

Library of Congress Catalog Card

Number: 56-12866

PRINTED IN THE UNITED STATES OF AMERICA By Waverly Press, Inc., Baltimore 


\section{Preface}

T $N$ THE PAST SEVERAL YEARS considerable progress has been made in the clarification of the problems of neural activity and of a host of ancillary physical and chemical events in living cells. Investigators in many fields from biology through chemistry, physics, mathematics, philosophy and psychology, have contributed new observations and concepts. They have drawn upon one another's knowledge and experience in attempts to refine existing experimental techniques and arrive at more accurate estimates of the properties and interrelationships of particular structures, mechanisms and processes at levels from the grossest complexity of the organism in toto to the most microscopic and sub-microscopic complexities of molecular structure and orientation.

Consequently, it was felt that there was need for some discussion of stimulusresponse problems not only insofar as existing data are concerned, but more particularly in relation to whether or not present experimentation and concept are leading to better understanding of the crucial problems underlying neuronal behavior.

The existence of certain transient phenomena in nerve cells forms the basis for their physiology and confronts the investigator with certain sorts of problems which, although they are not unique to this field, are representative of the difficulties involved in relating biological function to molecular interaction. The reduction of experimental observations to a molecular level must involve, first of all, a recognition that a rather small number of molecular units are involved in the events leading to the initiation of excitation in an excitable system, and that, therefore, the possibility exists that ordinary statistical considerations may not apply.

In seeking both the proper questions and answers, one is confronted at the outset with the initial way station of reception-the input to the nervous system. The major questions asked are how, in general, physical stimuli are transduced into nervous excitation ; and is the principle reaction chemical or physical? In other words, how are stimuli transformed into meaningful, informationcarrying nervous messages? It should be pointed out that while there is no requirement that transduction imply some analogy with mechano-electrical devices, it is suggested that an important property which must be inherent in a receptor is some means of converting the energy incident upon it into nervous excitation. The second question, which appears to stress a somewhat unreal dichotomy, is less clear in that a distinction between biophysical and biochemical reactions is less and less obvious as the units under consideration approach molecular dimensions.

Impulses from the receptors are passed on to aggregates of neurons in the central nervous system. At this level one would like to know if any progress has 
been made in our ability to explain observed properties of neurons in the aggregate on the basis of the differential biochemistry of the cells involved? Can we discuss the relationships of chemical structures to integrated activity or must new experimental paths be initiated which might be more productive than those now being pursued? It has been apparent for some time that not all cerebral neuronal groups, for example, behave similarly. At least part of the differential sensitivity of different types of neurons to drugs, anoxia and other environmental factors is related to either quantitative or qualitative differences in the sequence of metabolic reactions that are going on in the various cells. Nonetheless, present evidence forces one to the conclusion that certain underlying mechanisms of response are common to neurons in a wide variety of circumstances. The questions remain as to the role of biochemical reactions not only in these general responses, but also in the response mechanisms by which groups of neurons communicate with one another and do permit the organisms to be aware and well informed.

There remains for this symposium one further area which is somewhat more specific-namely, consideration of the physiological membrane. Any understanding of the various processes involved in nervous excitation devolves ultimately upon a model of some sort that represents a membrane. What is the nature of the membrane alteration that permits excitation or depression? In other words, how is molecular orientation in the membrane related to alterations in activity? In some respects this question differs from the others in that it is unlikely that the actual events occurring in the membrane can be determined directly by experimental means; rather, our understanding of such events appears more likely to be deduced from a series of models of greater and greater refinement, incorporating more and more of the phenomena actually observed. Whether there is any possibility of developing a model at present is a question that surely deserves to be examined. In addition, can any statement be made concerning the interrelationships of membrane structure and properties to physical and chemical events within the neuron? These are the fundamental mechanical questions which must be answered before understanding can be reached of the special faculties of neural tissue to receive and store information which can be communicated to and used by the organism in the proper time.

R. G. Grenell

L. J. Mullins 


\section{Table of Contents}

\section{Receptors}

1. Chemoreceptor Mechanisms. Vincext G. Dethier........... 1

2. Visual Receptors as Biological Transducers. E. F. MACNichol, JR.. 34

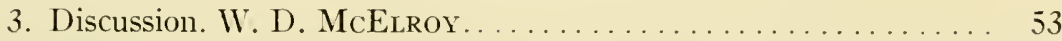

II. Aggregates of Neurons

1. Affinity for Wool as an Indicator of Neuropharmacological Activity. Roland Fischer. . . . . . . . . . . . . . . . . . . . . . . 63

2. Oxygen Utilization in Sympathetic Ganglia. I. Effects of Anesthetics. II. Substrates for Oxidation at Rest and in Activity. Martin G. Larrabee and Paul Horowicz. . . . . . . . . . . . . . . . . 84

3. Discussion. Robert G. Grenell. . . . . . . . . . . . . . . . . 108

III. Membranes of Neurons

1. The Structure of Nerve Cell Membranes. Lorin J. Muldins . . . . 123

2. Discussion. Philip W. Davies . . . . . . . . . . . . . . . . . 157

IV. Summary

Robert G. Grenell. . . . . . . . . . . . . . . . . . . 167 


\section{Participants}

\section{Chairman}

Ralph Gerard, Center for Advanced Study in the Behavior Sciences, University of Michigan, Ann Arbor, Mich.

Philip IV. Davies, The Thomas C. Jenkins Department of Biophysics, The Johns Hopkins University, Baltimore, Md.

Vincent G. Dethier, Department of Biology, The Johns Hopkins University, Baltimore, Md.

Roland Fischer, Department of Public Health, University Hospital, Saskatoon, Saskatchewan, Canada.

Robert G. Grexell, Psychiatric Institute, School of Medicine, University of Maryland, Baltimore, Md.

Martin G. Larrabee, The Thomas C. Jenkins Department of Biophysics, The Johns Hopkins University, Baltimore, Md.

Edward F. MACNichol, Jr., The Thomas C. Jenkins Department of Biophysics, The Johns Hopkins University, Baltimore, Md.

William D. McElroy, McCollum-Pratt Institute, The Johns Hopkins University, Baltimore, Md.

LORIn J. Mullins, Biophysics Laboratory, Purdue University, Lafayette, Ind. 


\section{Foreword}

T T $T^{\text {Ith }}$ this volume the American Institute of Biological Sciences begins a new symposium series. This venture has not been undertaken lightly. There are many - perhaps too many-such publications today. Almost every organization feels it must sponsor a program of publishing symposia. Why, then, should the AIBS add still another symposium series? Are there any compelling reasons why we should publish symposia?

First, through the generous provisions of a contract which the AIBS has with the Office of Naval Research, these published symposia can be made available to AIBS members at a considerably lower price than would be possible if they were published commercially. In effect, the Office of Naval Research is subsidizing the preparation and publication of these symposia to a considerable extent. Second, this series will allow the publication of biological symposia of significance, but which are, perhaps, of limited appeal. Third, a society which belongs to the AIBS can publish symposia through the Institute more economically and more efficiently than it could do itself.

A great deal of thought, preparation, and just plain work goes into the production of a series of this type. Many people have contributed. Due credit should be given the Office of Naval Research for its sponsorship of these symposia through its contract with AIBS. Mr. Irvin Mohler made all arrangements for the Symposium on behalf of AIBS and ONR. The officers of the AIBS have been most enthusiastic about this new venture. The members of the AIBS Editorial Board have given valuable advice in the plans as they have developed. Dr. Robert Grenell, one of the two Editors, because of his close proximity to Washington in nearby Baltimore, has been most helpful and generous with his time. The cooperation and assistance of Mr. Francis Harwood of the Waverly Press has been greatly appreciated. Finally, perhaps the majority of credit should go to Mrs. Ileen E. Stewart, Editor of AIBS publications, through whose efforts this series has finally seen the light.

Hiden T. Cox

Executive Director

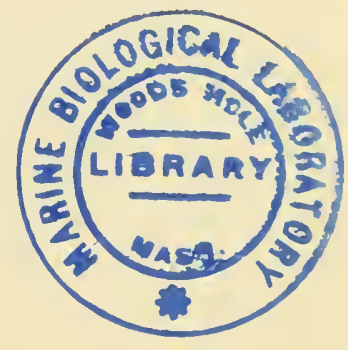




\title{
RECEP'TORS
}

\section{Chemoreceptor Mechanisms}

\author{
V. G. Dethier
}

Department of Biology, The Johns Hopkins University, Baltimore, Maryland

\section{Introduction}

7 he Following Two QUESTIONS, which were propounded by the originators of this symposium, represent the starting point and the ultimate goal of much of the research which involves stimulating sensitive tissue: How are physical stimuli transduced into nervous excitation? Is the ultimate reaction biochemical or biophysical? Insofar as stimulation by chemicals is concerned there is no tissue more appropriate for investigation than the tissue which is specialized by nature to receive chemical stimuli which play an indispensable role in the daily economy of living, in short, the chemoreceptors. In our ignorance it is still expedient to refer to the principal chemical senses as taste and olfaction. The following discussion will utilize this distinction; and, since greater attention has been bestowed by experimenters on the sense of taste, the major emphasis here will be placed on that sense. The discussion will, moreover, attempt to adduce from experiments conducted on the two most extensively studied groups of animals, mammals and insects, evidence for a general theory of action of chemical stimuli.

\section{Taste}

\section{Mammals}

Taste in mammals is subserved by cells within papillae situated principally on the tongue. Within the papillae the receptors are gathered together in groups, the taste buds. Within the buds lie a variable number of cells (Fig. 1). In man it is generally believed that there are four primary taste modalities, sour, salt, bitter, and sweet, and that most other taste sensations are compounded of these. The earliest suggestion that there might be specific receptors for the separate primary tastes derived from the observation that different areas of the tongue were differentially sensitive to the four qualities. Topical application of solutions 

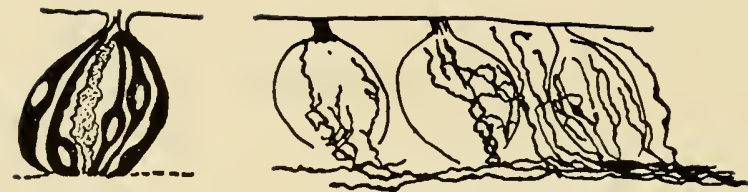

Sections through Taste-Buds of the Rabbit, Prepared by Golgi Method l.eft (after von Lenhossék), showing sensory cells and a "sustentive" element; right (after Retzius), showing terminal fibers.

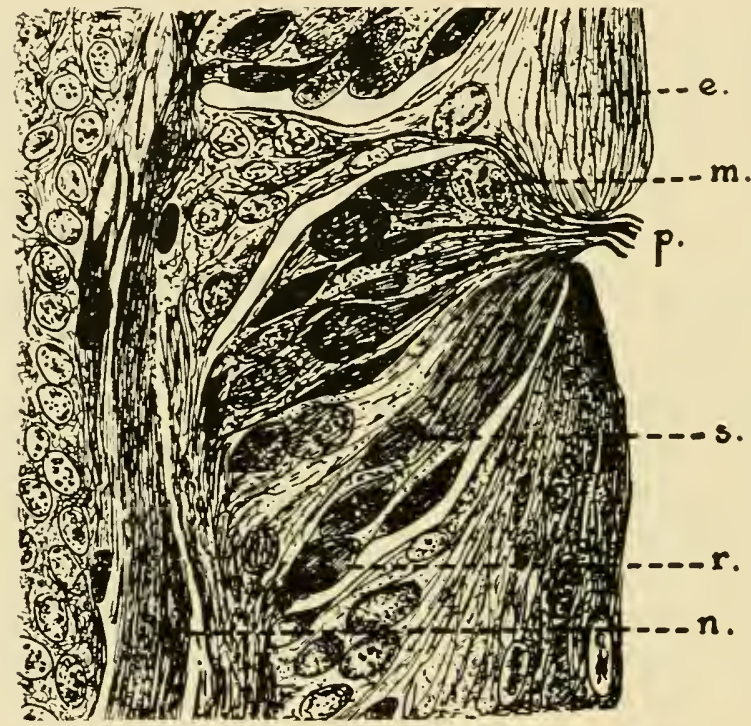

Section through One of the Taste-Buds of a Papilla Foliata of the Rabbit $e$, superficial epithelial cells; $m$, leucocyte containing granules; $p$, gustatory pore; $s$, gustatory cell; $r$, sustentacular cell; $n$, nerve fibers.

(From Quain, after Ranvier, copied in Howell.)

FIG. 1. Taste bud in the tongue of a rabbit (from Crozier, 1934)

then revealed that a single papilla might respond to one taste, all tastes, no taste, or a combination of tastes. It was not, however, until electrophysiological techniques were developed that it became at all possible to determine to what extent each single receptor was sensitive to the hundreds of compounds which are adequate stimuli for the tongue as a whole. And even now the situation is far from clear, because a single fiber may innervate more than one receptor cell. Where a fiber is seen to respond to either of two substances placed on the tongue, 
TABLE 1

Scheme illustrating possible relationship between afferent impulse pattern and evoked taste sensation in the cat (Cohen, Hagiwara, and Zotterman, 1955)

\begin{tabular}{c|c|c|c|c|c}
\hline \multicolumn{1}{c|}{ Stimulus } & $\begin{array}{c}\text { Water } \\
\text { Fiber }\end{array}$ & Salt Fiber & Acid Fiber & $\begin{array}{c}\text { Quinine } \\
\text { Fiber }\end{array}$ & Sensation Evoked \\
\cline { 1 - 2 } $\mathrm{H}_{2} \mathrm{O}(\mathrm{salt} 0.03 \mathrm{M}) \ldots \ldots \ldots$ & + & 0 & 0 & 0 & water \\
$\mathrm{NaCl}(0.05 \mathrm{M}) \ldots \ldots \ldots \ldots$ & 0 & + & 0 & 0 & salt \\
$\mathrm{HCI}(\mathrm{pH} 2.5) \ldots \ldots \ldots \ldots \ldots$ & + & + & + & 0 & sour \\
Quinine $\ldots \ldots \ldots \ldots \ldots \ldots$ & + & 0 & 0 & + & bitter \\
\hline
\end{tabular}

there remains a question of whether two specific receptors are involved or a single less specific one. Pfaffmann (1941) first demonstrated by recording from single fibers in the chorda tympani nerve of the cat that there were at least three physiologically different types of receptors (the term "receptor-unit" as applied by Beidler, Fishman, and Hardiman (1955) is preferable). One type responded only to acids, a second to acids and salts, and a third to acids and quinine. No fibers were then found which responded when sugars were applied to the tongue. Subsequently, "sweet" fibers were demonstrated (Beidler, 1952); and recently Cohen, Hagiwara, and Zotterman (1955) further characterized four physiologically different fibers. Their findings are summarized in Table 1.

In the frog, Zotterman $(1949,1950)$ and Andersson and Zotterman (1950) found some fibers which responded specifically to water and to dilute $\mathrm{NaCl}$ $(<0.025 \%)$, some which were specific for acids, and others which responded to hypertonic $\mathrm{NaCl}$ and $\mathrm{CaCl}_{2}$. In the chorda tympani of the dog, Andersson et al. (1950) detected fibers which were specific for sugars. In the rat, Beidler (1953) found fibers which responded specifically to salt, to salt and acid, and to sugar.

The foregoing results clearly indicate that there is a degree of true specificity in taste receptors of mammals and that there exists at the peripheral level a basis for the four modalities. There is evidence, furthermore, that the sensitivity of the several receptors represents a specialization of these cells and is not merely a consequence of exposure and opportunity. Beidler (1953) has shown, for example, that salt receptors in the rat have a lower threshold for $\mathrm{NaCl}$ $(0.002 \mathrm{M})$ than other receptors innervated by the lingual nerve $(+1.0 \mathrm{M})$ and than bare axon.

Granting, therefore, that there may be found in the mammal taste receptorunits which are specifically sensitive to acid, salt, acid and salt, acid and quinine, water, and sugar, what further information can be adduced concerning the characteristics of these units? Most of the experimental work which has been done has involved stimulation of large populations of receptors, i.e., areas of the tongue or individual papillae. Some of the electrical recording has been con- 


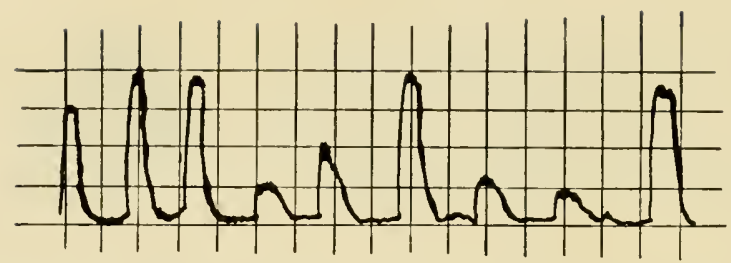

FIG. 2. Integrated electrical response of chorda tympani to various $0.1 \mathrm{M}$ salt solutions flowed over tongue of rat: $\mathrm{NH}_{4} \mathrm{Cl}, \mathrm{LiCl}, \mathrm{NaCl}, \mathrm{KCl}, \mathrm{CaCl}_{2}, \mathrm{NaCl}, \mathrm{RbCl}$, $\mathrm{CsCl}, \mathrm{NaCl}$. Test solutions are interspaced with water rinses. Time scale: 1 large division $=20 \mathrm{sec}$. (Redrawn from Beidler, 1953).

cerned with few-fiber preparations or with total-fiber integrated response (Fig. 2) (Beidler, 1953). Information derived from these approaches has revealed facts of the following sort.

There is general agreement insofar as the acid taste is concerned that an important constituent of the stimulus is the hydrogen ion. However, if this ion were the sole factor, all acids would be expected to taste equally sour at the same $\mathrm{pH}$, and this is not so. Some workers feel that the discrepancy is to be accounted for on the basis of interaction with saliva; others do not agree (Pfaffmann, 1951, Beidler, 1952). It is clear that there is no simple correlation with $\mathrm{pH}$, and, by analogy with the salts, it is not at all unlikely that the anion may have a contributory effect. In the case of insects, where there exist acid receptors unencumbered with saliva, there is again no simple correlation with $\mathrm{pH}$ (Chadwick and Dethier, 1947). Furthermore, as Beidler (1952) has pointed out, experiments with buffered solutions, designed to isolate the effect of the hydrogen ion, are confounded by the existence of receptors which are sensitive to salts as well as to acid.

Practically all salts are stimulating. Their stimulating efficiency in a variety of animals has been expressed as series of values based upon threshold measurements, saltiness of taste, etc. There are serial differences from one species of animal to the next and differences depending upon the criterion of response which is chosen. With regard to species differences, Beidler, Fishman, and Hardiman (1955) have reported the results of recording electrically the response of the chorda tympani to chemical stimulation of the tongue of rats, guinea pigs, rabbits, cats, dogs, hamsters, and racoons. Marked differences exist in the ability of the taste receptor-units ( $=$ a group of cells innervated by one single chorda typani fiber) to respond to a number of substances. The rodents respond well to $\mathrm{NaCl}$ compared to $\mathrm{KCl}$, whereas the opposite is true with the carnivores. Animals of both orders respond well to $\mathrm{NH}_{4} \mathrm{Cl}$. The hamster and guinea pig are easily stimulated by sucrose, whereas the cat is not.

For many species the cations can be arranged in increasing order of stimulat- 
ing effectiveness as follows: $\mathrm{Li}<\mathrm{Na}<\mathrm{Ca}<\mathrm{K}<\mathrm{NH}_{4}$. The anions are frequently arranged: $\mathrm{SO}_{4}>\mathrm{Cl}>\mathrm{Br}>\mathrm{I}>\mathrm{HCO}_{3}>\mathrm{NO}_{3}$. It is probable that both the cations and the anions play a role in stimulating the receptor. The order of efficiency of the cations as given above is also that of the ionic mobilities and the partition coefficients as well. The arrangement of the anions is less clear. The activity of the divalent cations is anomalous in that they do not fit into any of these series. The work of Beidler with the rat has shown that the divalent salts are different from the monovalent salts in several respects. Hodgson (1951) has also noticed this difference in tests with the water beetle, Laccophilus. In man many of the divalent salts are bitter. Moreover, each salt has a different and recognizable taste and so must stimulate different receptors or combinations of receptors.

Hardly any experiments have been undertaken to test the effect of simple organic compounds on the taste receptors of mammals. The lack may stem in part from complications introduced by the concomitant olfactory stimulation effected by most of the compounds in this category. However, data which exist for the homologous aliphatic alcohols and glycols (Figs. 3 and 4) indicate that the thresholds (molar) decrease logarithmically with increasing chain length (Renqvist, 1920; Dethier, 1951b; Gavaudan, 1948, 1951; Poussel, 1951). In the case of organic salts, Beidler (1954) found that there was a decrease in effect with chain length; however, he tested sodium salts only up to butyrate; and data of Dethier (Fig. 5) with longer series of sodium, lithium, and barium salts acting on insects show that beginning with the five carbon compound there is a logarithmic decrease similar in all respects to that observed with non-polar homologues.

The outstanding feature of all of the data accumulated from tests with sweet and bitter substances has been the remarkable nonconformity of the physiological effect with any single chemical or physical property of the sort recorded in whole or in part for the non-sweet compounds. On the contrary, there is to be noticed a much more precise dependence upon specific chemical configurations. This dependence is true of the bitter as well as the sweet substances. For example, ortho-tolylurea is tasteless, meta-tolylurea is bitter, and para-tolylurea is sweetish (Beidler, 1952). Other equally small changes in molecular structure are reflected as pronounced physiological changes. Stereo isomers also have different tastes. For example, D-asparagine is sweet; L-asparagine is tasteless. In the case of insects, D-arabinose is nearly five times more stimulating than L-arabinose (Hassett, Dethier, and Gans, 1950).

\section{Insects}

All of the information summarized in the foregoing paragraphs can be multiplied and elaborated a hundredfold. It is well to remember, however, that in 


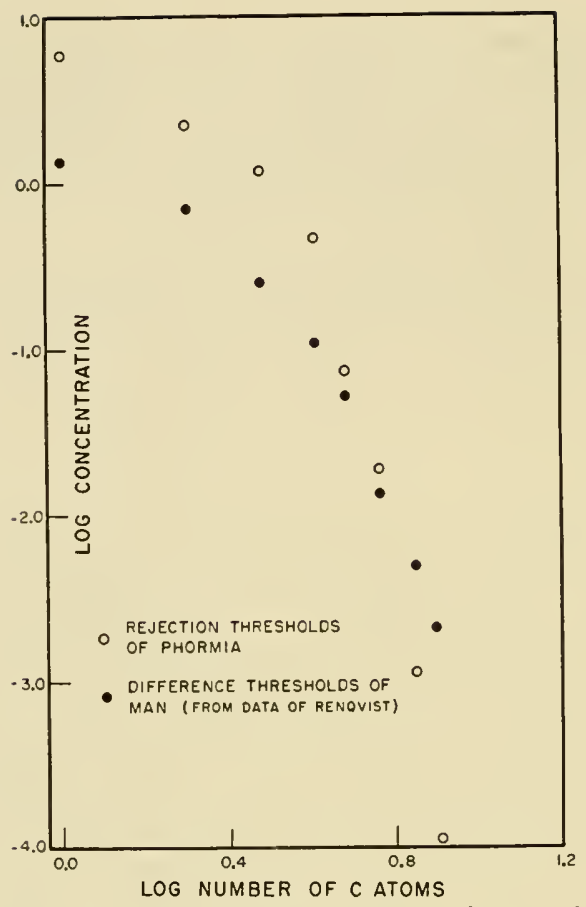

Fig. 3. Comparison of relative effectiveness of homologous alcohols in stimulating tongue of man and tarsal receptors of the blowfly (Dethier, 1951b).

all but a very few cases the information was derived from stimulation of populations of mixed receptors, and to what degree the information can be extrapolated to single receptors remains to be seen. Until very recently the situation in insects was the same. A great deal of information had been amassed as a result of stimulating receptor fields. Just as the criteria with mammals were subjective or behavioral, so in insects they were behavioral. In general the picture which emerged was one of two taste modalities, acceptable and unacceptable. (Some experiments in progress now suggest that this conclusion may be an over-simplification.) Stimulation of insect receptors by electrolytes (Frings, 1946, 1948; Frings and O'Neal, 1946; Hodgson, 1951) revealed that, with minor exceptions, the stimulating efficiencies of cations and anions follow the pattern already given. Furthermore, there was evidence that both ionic species are contributing to the event. The similarity of acid stimulation in insects to that in man has already been mentioned. In the case of sugars the high degree of molecular specificity already noted with man is found to exist in insects in as high or possibly in a higher degree (von Frisch, 1935; Hassett, Dethier, and Gans, 1950; Dethier, 1955). Data relative to the stimulating effects of organic compounds are far more complete for insects than for man (Dethier, 1953). Here also there is a 


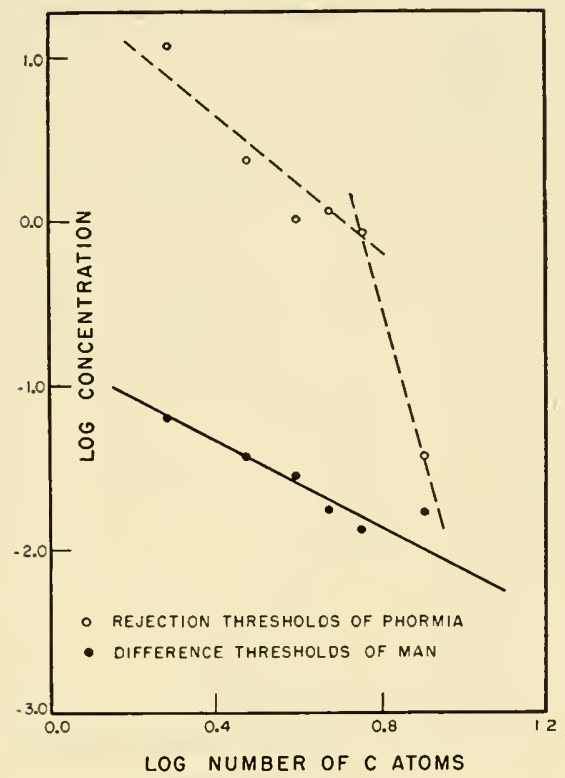

Fig. 4. Comparison of relative effectiveness of homologous glycols in stimulating tongue of man and tarsal receptors of the blowfly (Dethier, 1951b).

general similarity to the results obtained with man in that the threshold decreases logarithmically with increase in chain length. For insects this is not an even decrease. In the case of homologous alcohols the curve shows a break in the region of the four and five carbon compound (Fig. 6). In series of aldehydes and ketones the break is in the region of the three carbon homologue (Fig. 7 ). In the glycol series there is a break in the region of the six carbon compound. When large chain polymers are employed as stimuli, the break persists but in the opposite direction, i.e., the increase of stimulating effect with increasing chain length is less pronounced for the higher members than for the lower ones (Fig. 6). In short, polymer mixtures do not react the same way as pure compounds.

It is possible to relate threshold rather accurately to chain length and to make precise predictions on the basis of these relationships. But of all the colligative properties of homologous compounds the only one which permits bringing members of all series into one frame of reference is solubility. The threshold is directly proportional to the water solubility (Fig. 8) (Dethier and Chadwick, 1950). Additional evidence that solubility is of importance in this connection has been presented by the work of Dethier (1951a), which shows that the measured thresholds of alcohols are altered as the alcohol is presented as an aqueous solution, a glycol solution, a mineral oil solution, (Fig. 9). When threshold values 


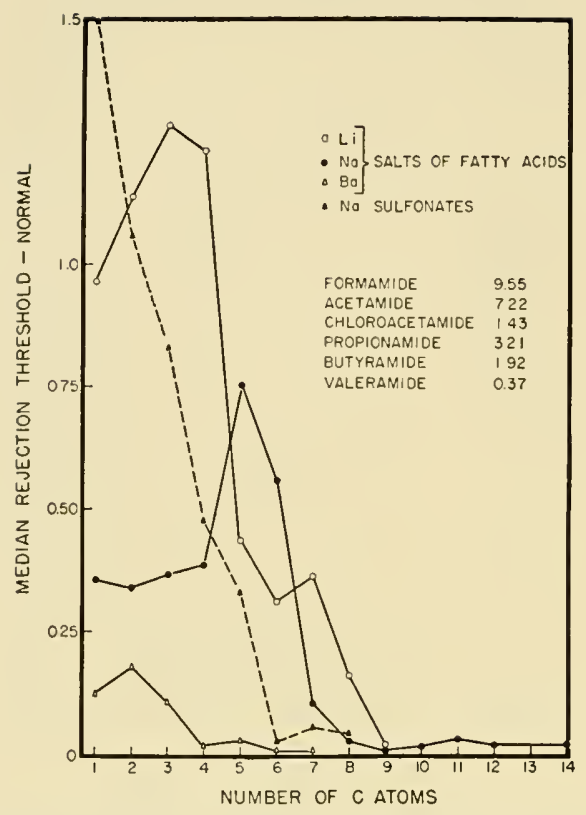

FIG. 5. Relation between the stimulating efficiency of organic salts and the chain length of the anion.

are expressed as thermodynamic activities rather than as moles, the differences between successive homologues of a series are not so marked, but a plot of the $\log$ of these values against the $\log$ of activity coefficients (Fig. 10) does not produce a straight line of the sort that one is accustomed to expect from parallel experiments on narcosis (for a complete discussion consult Ferguson, 1951; Brink and Posternak, 1948; Dethier, 1954b).

An attempt was made to obtain with human beings some data comparable to those just outlined above even though the statement is frequently made that man tastes only those substances which are in aqueous solution. Tests revealed that it was possible to measure taste thresholds for alcohols dissolved in mineral oil (Dethier, 1952). As was the case with insects, man's thresholds for the homologous alcohols in oil are nearly but not exactly identical for each homologue. A plot of the oil threshold/water threshold ratio against the activity coefficient (Fig. 11) indicates that the members seem to fall into two groups, the higher members being in one group and the lower in another. If the threshold ratios are plotted against the oil/water partition coefficients, it can be seen that the two sets of values are not the same (Fig. 12). This lack of complete agreement may be attributed either to the interference of saliva and to the fact that one is working with large populations of mixed receptors, or it may indicate that oil/water partitioning is not the limiting factor. 


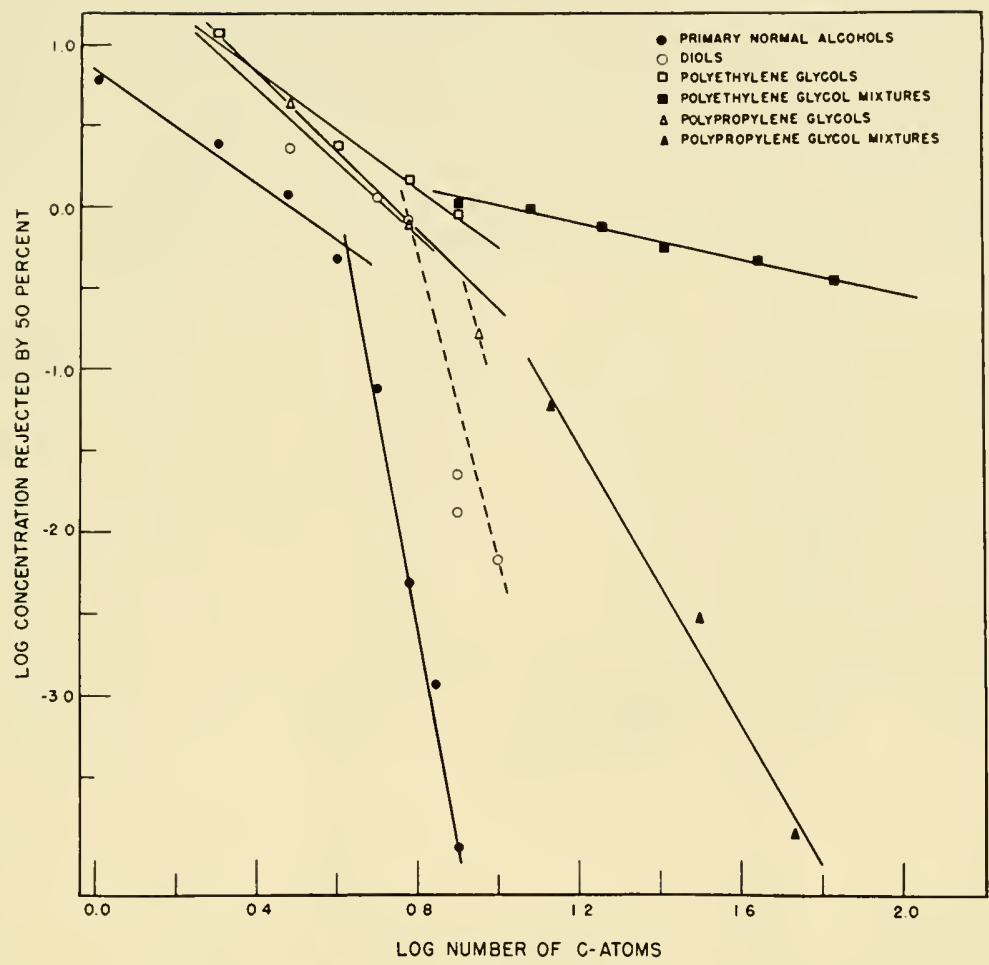

Fig. 6. The relation between the rejection thresholds of alcohols and glycols by the blowfly and the chain length (Dethier and Chadwick, 1948b).

With insects one tremendous advantage has recently been granted us. It is now possible in the blowfly, Phormia regina, to work behaviorally and electrophysiologically with a single-receptor preparation. The histology of the receptors has been studied in great detail (Dethier, 1955). It is possible to stimulate a single receptor and elicit a complete normal behavioral response (Dethier, 1955) and a clear electrical response (Hodgson, Lettvin, and Roeder, 1955). The receptor organ concerned is analogous to a single mammalian taste bud in that it contains more than one receptor. Two receptors have been demonstrated, but each is innervated by its own nerve fiber and each can be stimulated singly. The receptor organ is a hair (sensillum trichodeum) containing three bipolar neurons two of which send distal processes to the tips (Figs. 13 and 14) where they are clearly invested with a cuticular covering which possesses, however, permeability characteristics radically different from those of the cuticle on the rest of the hair. Furthermore, the cuticle at this point has a very low electrical resistance as compared to the rest of the hair which is nearly perfectly insulated. The process of the third neuron ends at the base of the hair and may not 


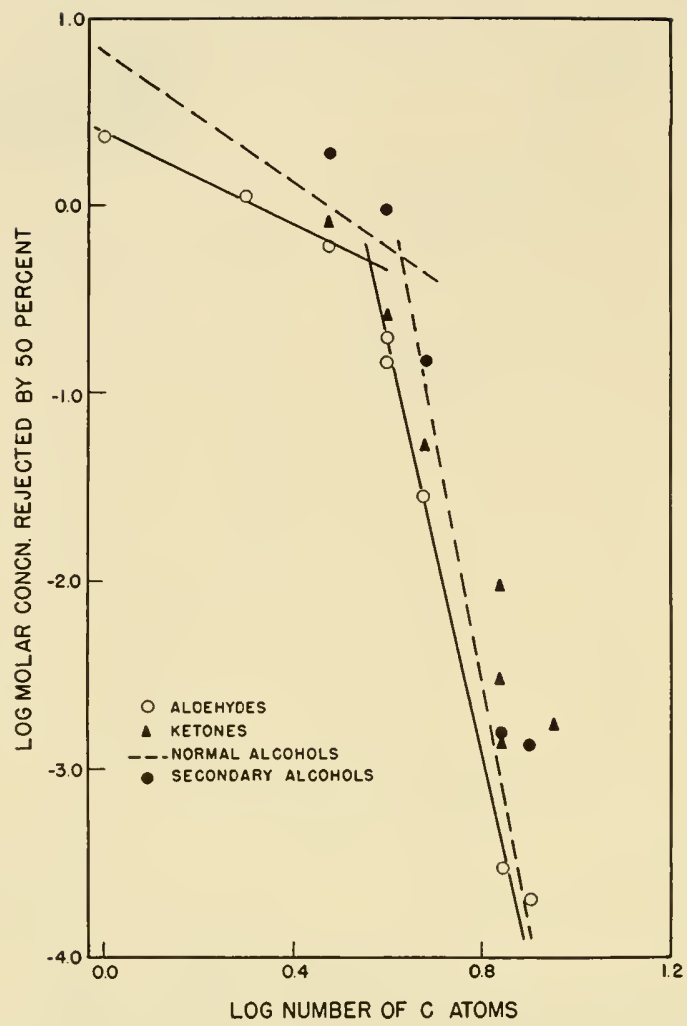

Fig. 7. The relation between the rejection thresholds of alcohols, ketones, and aldehydes by the blowfly and the chain length (Chadwick and Dethier, 1949).

be concerned directly with chemoreception, although this remains to be proven. The proximal processes of all three cells travel directly to the central nervous system without, as far as we know, synapsing. Here, then, is a primary neuron, one end of which is exposed to the stimulus and the other end of which makes its first connection deep within the central nervous system. This is indeed a convenient preparation to have at hand.

Only the tip of the hair, where the two fibers terminate in a minute papilla (Fig. 14), is sensitive to stimulation. A single hair is sensitive to every compound to which the animal as a whole is responsive provided that a threshold concentration can be applied. Stimulation by certain sugars elicits a behavioral response in the nature of a proboscis extension. Stimulation by non-sugar compounds elicits withdrawal of the proboscis; or it inhibits extension. A variety of tests, such as simultaneous stimulation of one hair with sugar and another with salt, show that the inhibiting compounds are truly stimulating. The elec- 
trical recordings of Hodgson et al. (1955) and similar ones obtained in our laboratory (Fig. 15) show clearly that compounds such as salts, alcohols, etc., fire one of the two fibers while sugars fire the other. Thus, by choice of stimulus one has available a single-receptor preparation. In contrast to the situation as it exists in mammalian tongues, there appear to be only two specific receptors in flies, one for sugar, the other for acids, salts, and various organic compounds. Of course, further extensive exploration is necessary, especially with reference to the role of the third neuron.

Studies have been started (Dethier, 1955) which show that most of the information regarding the nature and effects of stimuli as applied to receptor fields in the fly can be extrapolated to the single receptor. Thus, for example, the order of stimulating efficiency of cation series appears to be the same for the single receptor as for the animal as a whole, and the series resembles in many respects that found in certain mammals. The logarithmic decrease of threshold of homologous aliphatic compounds with the chain length, as recorded for the whole animal, applies also to single receptors. And, finally, the high degree of specificity for sugars is as characteristic of the single receptor as of populations of receptors.

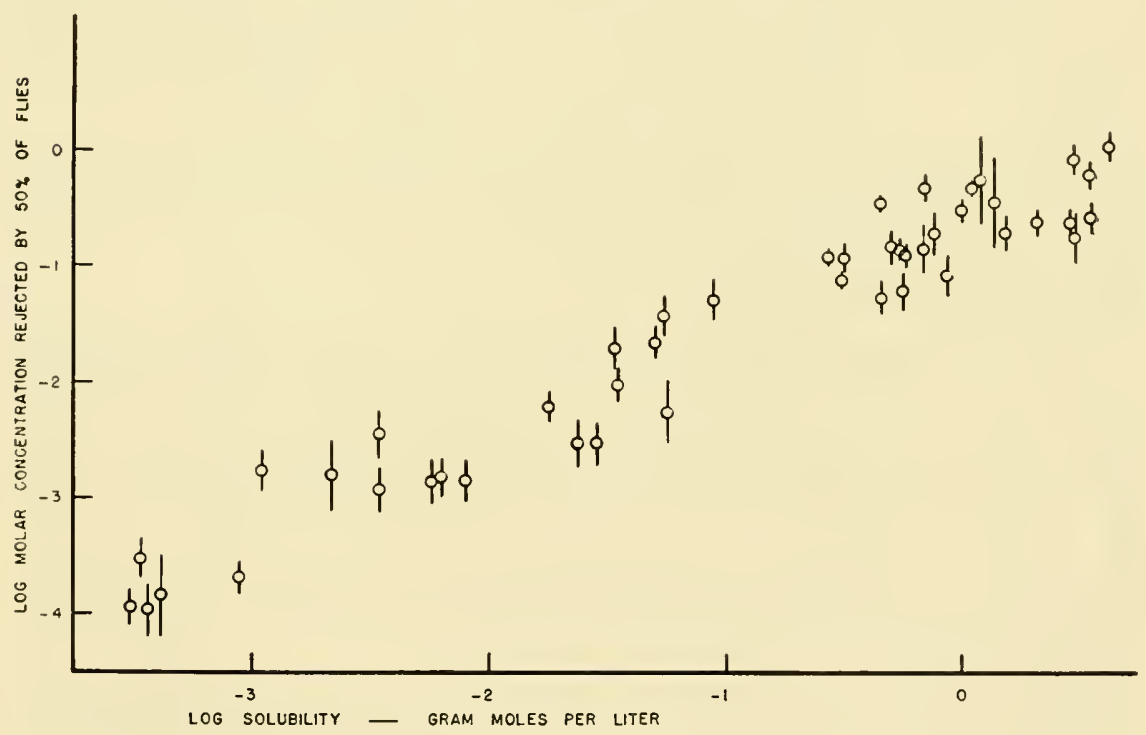

FIG. 8. Correlation between concentration required for stimulation and solubility of compounds at $25-27^{\circ} \mathrm{C}$. in water. Each of the 46 points represents a different aliphatic compound. The vertical lines represent the fiducial limits of the error of estimate of each determination at the 0.01 probability level (Dethier and Chadwick, 1950.) 


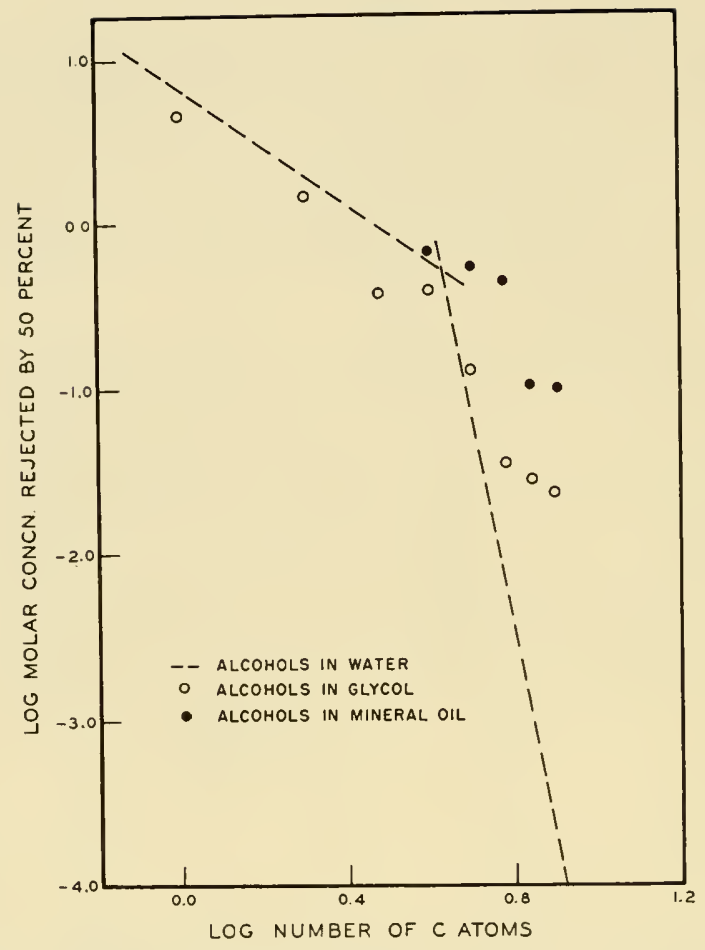

FIG. 9. Rejection thresholds of aqueous, glycol, and oil solutions of primary alcohols by the blowfly Phormia (Dethier, 1951a).

\section{Mechanism of Action of Sugars}

With the foregoing information derived from studies of various mammals and insects, and without losing sight of the fact that there are pronounced specific differences which eventually require explanation, we can attempt to draw some conclusions regarding the nature of the interaction between taste stimuli and chemoreceptors. First of all, since there is good evidence that there are specific types of receptors, it would seem intelligent to consider each apart. There is no reason to believe that all act similarly. It is certainly apparent that the sugar receptors of insects and the sugar/bitter receptors of mammals are basically different from non-sugar receptors. Some of the outstanding differences are these: (1) the sugar receptors are sensitive to highly specific chemical configurations; the other receptors react to an extremely wide variety of compounds; (2) the reaction of sugar receptors is slow compared to that of the other receptors; (3) the magnitude of electrical response of sugar receptors appears to be less than that of other receptors; (4) the amplitude of the potential in the sugar fibers is smaller than that of other fibers.

The really striking difference between the sugar receptor and all others is its 


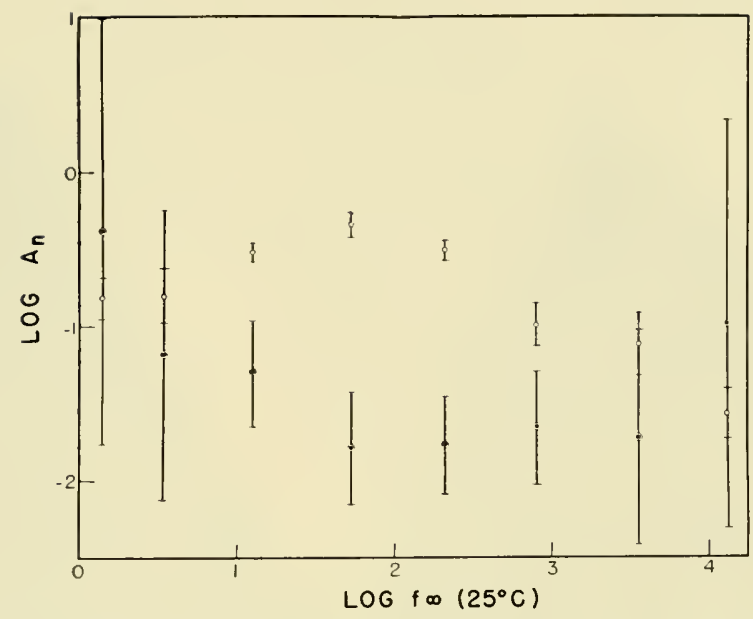

FIG. 10. Comparison in terms of thermodynamic activity of the stimulating effectiveness of the first eight normal alcohols acting in aqueous solution (open circles) on tarsal chemoreceptors and as gases (solid circles) on olfactory receptors. In each case the value represents a threshold of rejection. The vertical lines represent 2.575 standard errors for aqueous thresholds and 2 for vapors. (Dethier and Yost, 1952.)

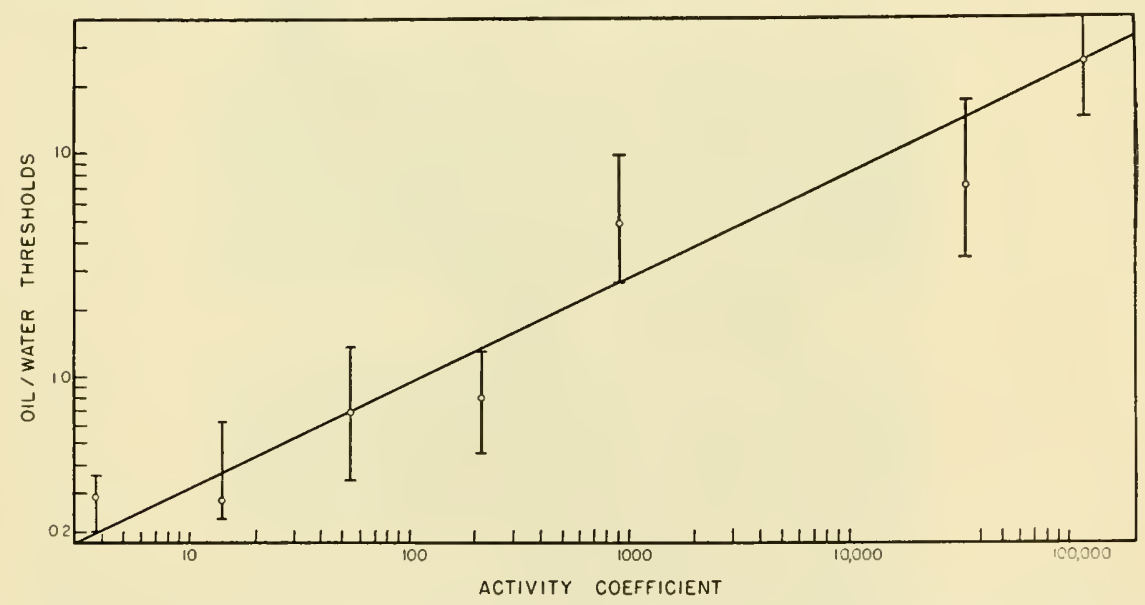

Fig. 11. Relation between human taste thresholds for alcohol, ethanol to octanol, and the activity coefficient. Threshold values are expressed as the ratio of the molar threshold in oil to the molar threshold in water.

specificity. We know little about the mammalian receptor as a unit, because only recently have potentials been recorded from it. From the stimulation of entire receptor fields we know that as a general rule sugars with an alpha glucoside linkage are sweeter than others; that alpha glucose is sweeter than beta glucose; that some D sugars are sweeter than the corresponding L sugars. For 


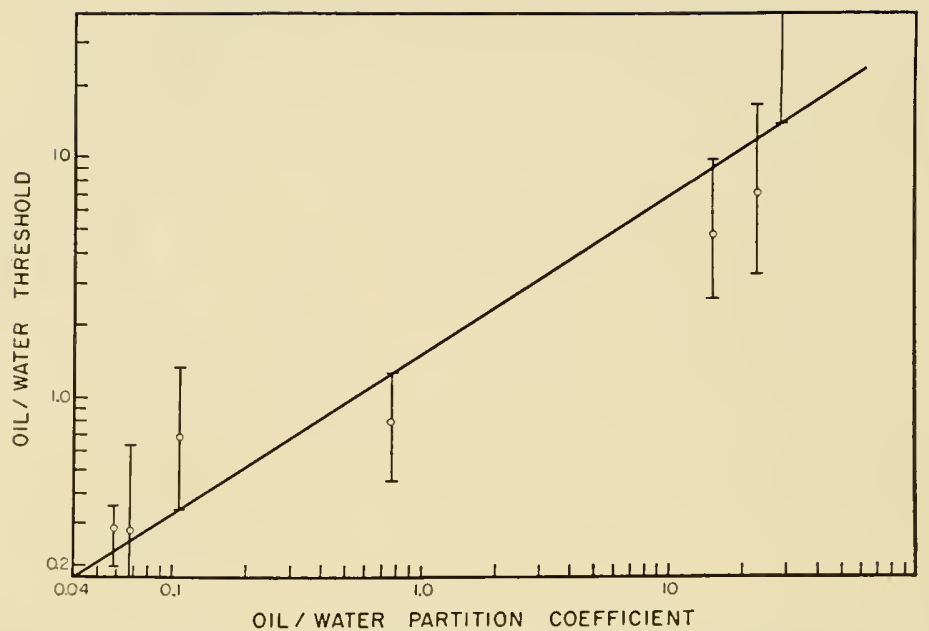

FIG. 12. Relation between human taste thresholds for alcohols, ethanol to octanol, and the oil/water partition coefficient. Threshold values are expressed as the ratio of the molar threshold in oil to the molar threshold in water.

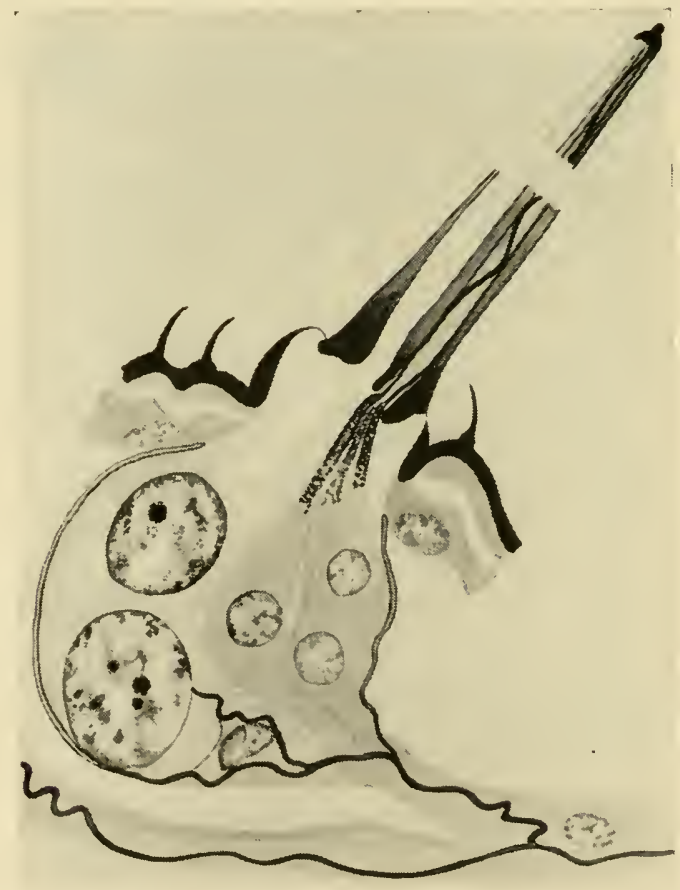

FIg. 13. Typical chemosensory hair of the blowfly, Phormia regina Meigen, showing the three bipolar neurons two of which send distal fibers to the terminal papilla by way of the thick-walled lumen of the hair (Dethier, 1955). 

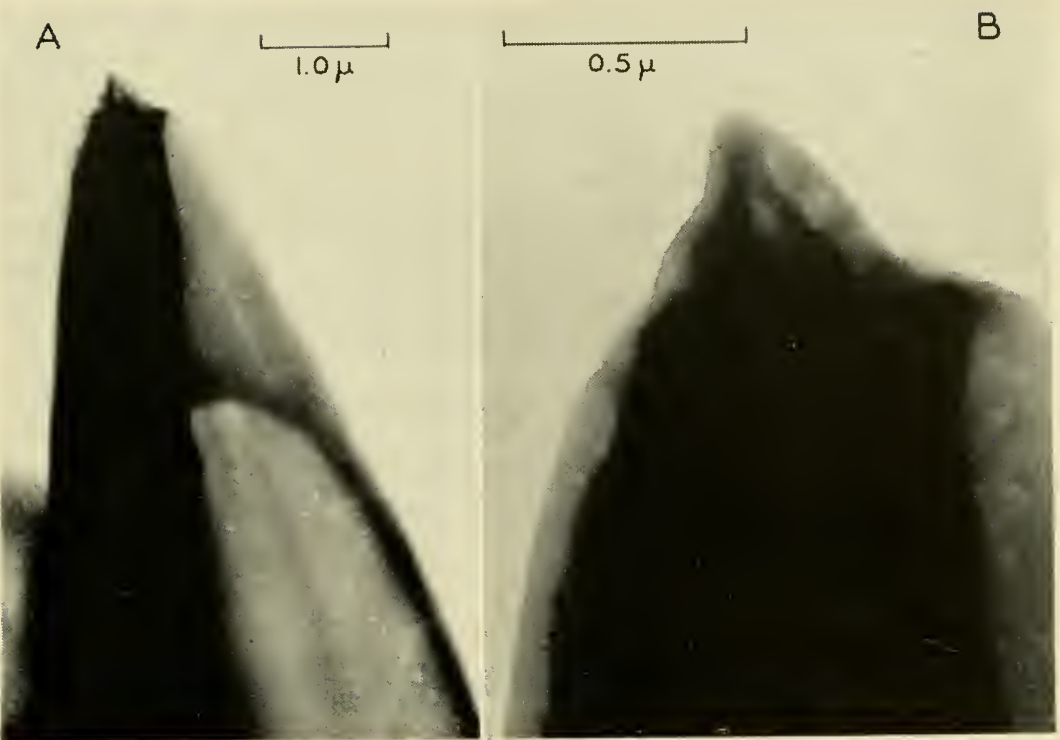

Frg. 14. Electron micrograph of labellar chemoreceptor of the blowfly, Phormia regina (through the courtesy of E. Kafig, Naval Medical Research Institute, and M. Wolbarsht, The Johns Hopkins University).
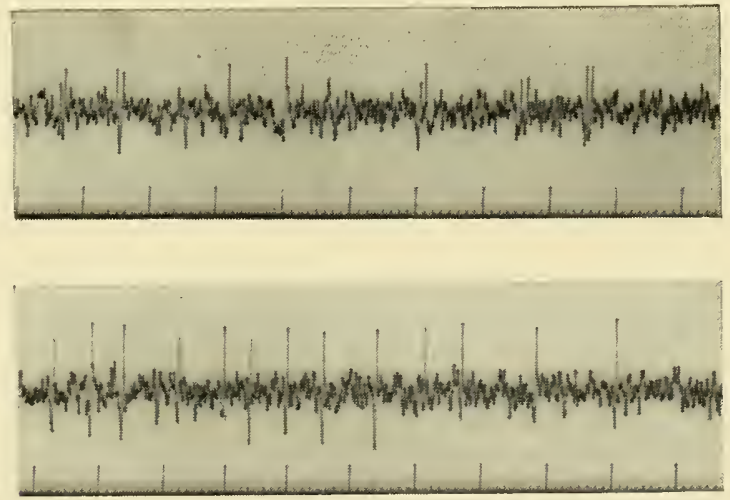

FIG. 15. Activity recorded from tip of a single labellar hair during chemical stimulation. Large spikes, $0.5 \mathrm{mV}$. Time marks, $0.01 \mathrm{sec}$. Upper record: stimulation with a mixture of $0.12 \mathrm{M}$ sucrose and $0.15+\mathrm{M} \mathrm{NaCl}$. Note paired spikes. Lower record: stimulation with $0.15+\mathrm{M} \mathrm{NaCl}$.

insects we know that very similar, if not identical, relationships can be demonstrated. These relationships strongly suggest that there is a highly selective receptor substance or site with which the sugar must react. The fact that response ceases almost immediately upon removal of the applied stimulating solution suggests that the sugar is rapidly removed from the receptor site. It can 
be assumed that four steps may be involved in the process of stimulation: (1) combination of sugar $(S)$ with receptor substance $(R),(2)$ initiation of polarization, (3) discharge of impulses in the sensory nerve, (4) removal of sugar. Four alternative hypotheses may be advanced by way of checking the mechanisms enumerated in the basic assumptions.

The simplest hypothesis is that sugar combines reversibly with receptor substance to form a complex in or as part of the receptor membrane. Formation of the complex results in depolarization of the membrane. Removal of the external source of stimulation causes removal of combined sugar:

$$
S+R \rightleftharpoons R S \rightarrow \text { depolarization }
$$

A variant of this process would involve combination of sugar with a receptor substance which is thereupon activated to elaborate a product capable of depolarizing the membrane:

$$
S+R X \rightleftharpoons R S X \rightarrow X \rightarrow \text { depolarization }
$$

Two alternatives of these hypotheses envision the removal of sugar by a side reaction involving chemical degradation of sugar.

$$
\begin{aligned}
S+R \rightarrow R S & \rightarrow \text { depolarization } \\
& + \\
D & \\
& \downarrow \\
& R+S_{1} \\
S+R X \rightleftharpoons R S X & \rightarrow X \rightarrow \text { depolarization } \\
& + \\
& D \\
& \downarrow \\
& R+S_{1}
\end{aligned}
$$

An outstanding feature of stimulation by sugars is that no change in threshold can be demonstrated to take place as the temperature of the stimulus is changed. Frings and Cox (1954) have observed significant threshold changes when the temperature of the entire organism (the fly, Sarcophaga bullata) is altered, but even under these conditions the changes are small. The values are: $0.14 \mathrm{M}$ at $19-21^{\circ} \mathrm{C} ., 0.06 \mathrm{M}$ at $27-29^{\circ} \mathrm{C}, 0.17 \mathrm{M}$ at $37-39^{\circ} \mathrm{C}$. Dethier (Figs. 16 and 17 ) was able to demonstrate in the case of the chemosensory areas of the ovipositors of crickets and certain parasitic Hymenoptera that there was very little change in reaction time with temperature. Hodgson and Roeder (in press) have shown that the frequency of impulses in the afferent nerve fiber increases rapidly with 


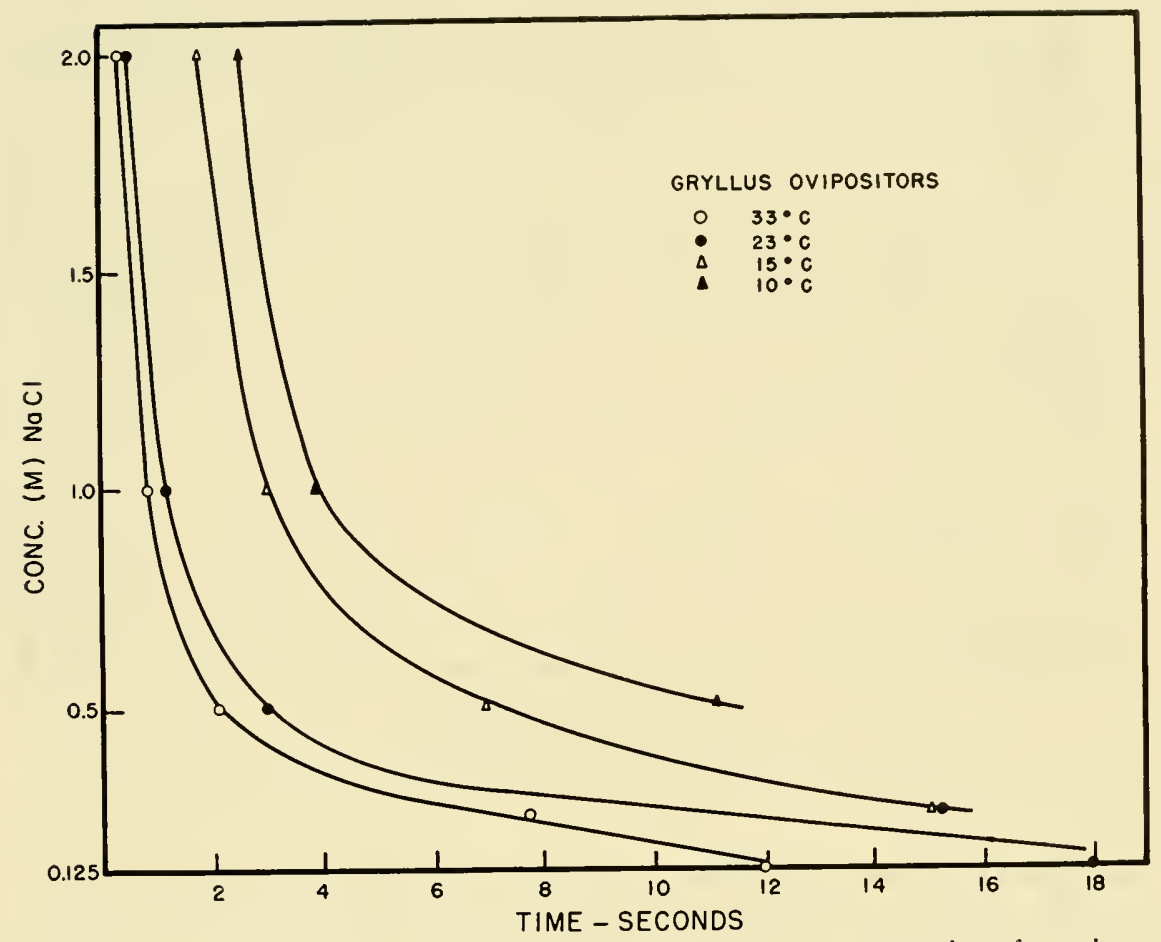

FIG. 16. Change in reaction time with temperature and concentration after stimulation of the ovipositor of the cricket Gryllus with $\mathrm{NaCl}$.

increase in temperature of the entire mouthpart. In this last experiment the cell body of the sensory neuron was subjected to the change in temperature. In all of the experiments conducted in this laboratory by Dethier and by Arab, in which the temperature of the stimulus, and presumably the receptor surface at the tip of the hair, was changed while the cell body and the afferent nerve fibers remained at room temperature, no significant alteration of threshold was detectable. Absence of a change of threshold under these conditions means that at all temperatures a given concentration of stimulus results in a given frequency of impulses in the afferent nerve. Furthermore, since the effect of the temperature change is at the receptor site some 300-400 microns distant from the cell body where the impulses are presumed to be generated, it seems reasonable to assume that the temperature effect, if any, would be acting on either or all of three processes and no others, namely: combination of sugar with receptor substance, removal of sugar, and depolarization. The absence of a demonstrable temperature effect suggests either (1) that the limiting process is temperature independent (or has a temperature coefficient approximating 1) or (2) that the net temperature effect of a reaction with a positive temperature coefficient and 


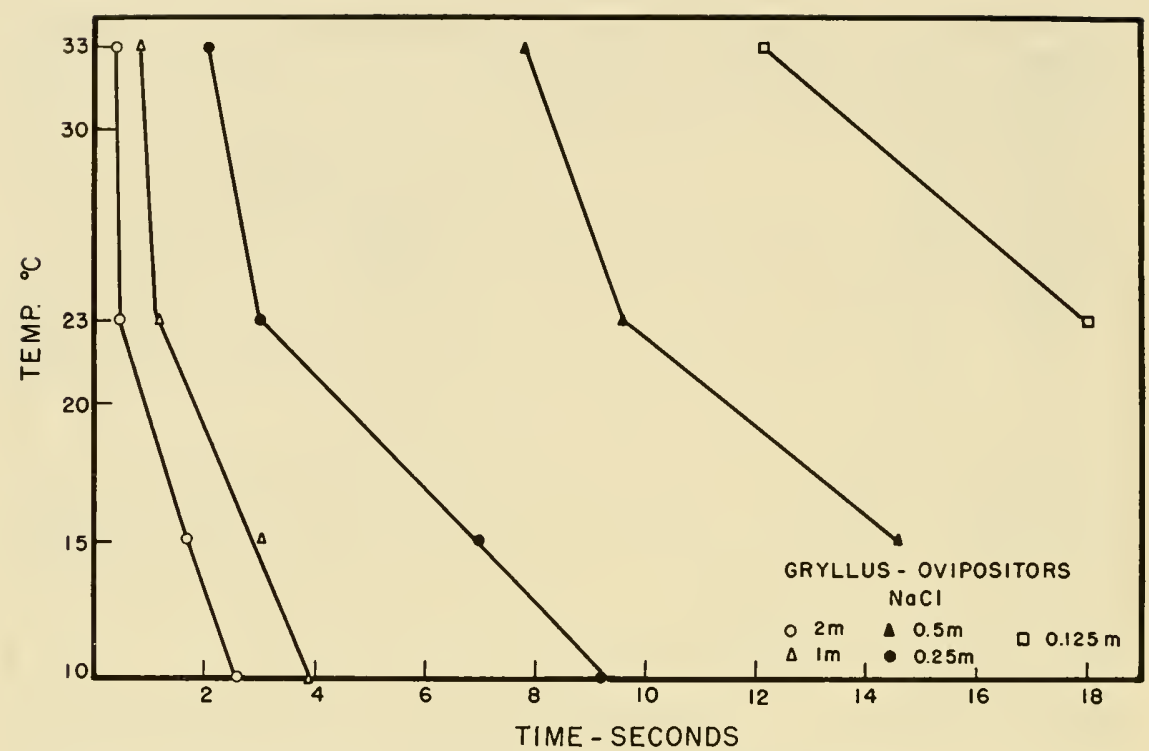

Fig. 17. Change in reaction time with temperature and concentration after stimulation of the ovipositor of the cricket Gryllus with $\mathrm{NaCl}$.

one with a negative temperature coefficient is negligible. Of the two alternatives the former seems the more likely. Acceptance of the former would rule out many enzymatic reactions as the limiting reaction. It would argue in favor of the first step $(S+R \rightleftharpoons R S)$ being one which involved binding forces of the order of van der Waals' forces, which fall off as the seventh power of the distance. The critical importance of molecular configuration is consistent with this idea in that attraction would depend upon close association between $S$ and $R$ with conformity of more than one point. The reaction would not be expected to be an all-or-none one but would be expected to exhibit gradations correlated with the goodness of fit. This expectation is realized in the finding that some sugars are excellent stimuli (low threshold), others good, others poor.

Another feature of stimulation by sugars is that it is not inhibited by any of the following inhibitors: phlorizin, fluoride, azide, iodacetate, cyanide. This finding indicates that the first step in stimulation probably does not involve any steps in the glycolytic cycle below those blocked by the agents listed.

Now, if the receptor sites (or substance) become filled and no sugar is removed, stimulation might be expected to continue in the absence of externally applied solutions until adaptation sets in. Since this occurrence has not been observed, it must be presumed that the sites are rapidly emptied. The most likely means of removal of a sugar such as glucose would be by a chemical reaction such as phosphorylation. It might be expected that application of a specific inhibitor to block this reaction would not prevent initial stimulation but 
might, by blocking emptying of the sites, inhibit repeated stimulation. Furthermore, the reaction might reasonably be expected to be temperature dependent, and subjection of the receptor to low temperature would block continued stimulation.

It is known, however, that the pentose fucose, which has been shown by Evans in this laboratory to be non-metabolizable by the fly, stimulates just as efficiently as glucose, and, upon removal of the applied solution, stimulation apparently ceases just as abruptly as in the case of glucose. Thus, a sugar like fucose apparently can be removed passively, and there is no need to postulate a separate mechanism for the removal of glucose. This conclusion would favor equations (1) and/or (2) as correct representations of the state of affairs. Although we are not at this time able to choose between equations (1) and (2), the evidence presently at hand certainly can be satisfactorily explained by the simpler hypothesis (1). Tentatively, then, it is proposed that stimulation by sugars, at least in insects, involves combination of the sugar molecule with a specific receptor substance or site by weak forces, such as van der Wraals', to form a complex which depolarizes the membrane, after which (or simultaneously) sugar is removed passively by a shift in concentration gradient.

\section{Mechanism of Iction by Salts}

The case for the salt receptors of vertebrates is different in several respects. Beidler (1954) has pointed out: (1) the reactions involved are in a time-independent state and stimulation is probably in thermodynamic equilibrium, (2) it is very rapid (approximately 50 msec.), (3) it is completely reversible, (4) cations and anions both enter the reaction although the magnitude of the response is determined chiefly by the cations, (5) a saturation level is reached, (6) receptors respond to many diverse substances. The first statement is based upon the observations that the response to $0.1 \mathrm{M} \mathrm{NaCl}$ remained constant during 10 minutes of continued stimulation and the magnitude of the response remained the same regardless of preceding stimulation by either higher or lower concentrations of salt. The second statement reveals a latent period shorter than that measured in behavioral studies, but this is to be expected. In the fly, for example, the time for a complete behavioral response is approximately 0.1 sec.; this value is not out of line. New data reported by Hodgson and Roeder (in press) show values of $\mathbf{5}-\mathbf{1 0} \mathrm{msec}$. Evidence for action by both cations and anions is seen in practically all studies made regardless of the species of animal. There is indirect evidence that attainment of a saturation level as described for the rat receptor occurs with the insect receptor as well (Dethier, 1955). Again, new data of Hodgson and Roeder show a stimulus concentration at saturation to be of the same order of magnitude (e.g. ca. $0.5 \mathrm{M} \mathrm{NaCl}$ ) for both insects and mammals. Attainment of saturation is well illustrated by the observation that in balancing salt stimulation against sugar stimulation there is a concentration 
of salt above which no increase of sugar will effect a balance. This "saturation level" is different for different compounds.

On the basis of some of the facts given in the preceding paragraphs and by making certain basic assumptions Beidler (1954) has proposed a theory of taste applicable to salt receptors. He has assumed that the stimulus reacts with some receptor substance and that the reaction obeys the mass action law. His second assumption is that the magnitude of response is directly related to the number of ions or molecules that have reacted with the receptors. An equation can be derived which relates magnitude of response to the concentration of the applied chemical stimulus. This is $c / R=\left(c / R_{m}\right)+\left(1 / K_{m}\right)$ where $c=$ concentration, $R=$ magnitude of response, $R_{m}=$ magnitude of maximum response, and $K=$ the equilibrium constant. The validity of the equation can be tested by measuring $c$ and $R$. Beidler found the agreement to be excellent. Now, knowing the equilibrium constant, he calculated the free energies of reaction from the expression $\Delta F=R T \ln K$ where $\Delta F=$ change of free energy, $R=$ the gas constant, and $T$ the absolute temperature. The low value which he found for $\Delta F$ (from -1.22 to -1.37 Cals./mole) for a series of sodium salts is taken to indicate that physical rather than chemical forces are involved in the interaction between the chemical stimulus and the receptors. This conclusion raises the question of what the receptor substance may be.

Beidler found that the magnitude of response in receptors of the rat varied little with change of temperature. Earlier studies of the effects of temperature on human taste thresholds had shown little consistency from one worker to the next or for any two substances. In this connection Pfaffmann (1951) had concluded that the temperature effects were complex and not indicative of a simple chemical reaction. The findings with respect to insects generally failed to show any convincing temperature effect on threshold (Dethier and Chadwick, 1948a). Beidler has argued that the small temperature dependence and the low values for $\Delta F$ suggest a reaction similar to those which occur with ion binding by proteins and natural polyelectrolytes. Some properties of the reacting groups of the ieceptor substance can be determined from a study of the effect of changing $\mathrm{pH}$. Since no such change could be demonstrated over the range 3.0 to 11.0 , one might conclude that the molecules of the receptor substance are strong acidic radicals. The relatively weak carboxyl radical of a protein, for example, cannot be considered as the reacting group. The phosphate and sulfate radicals of such natural polyelectrolytes as nucleic acids and certain polysaccharides are able to bind cations in a manner consistent with the properties of the receptors described (Beidler, 1954). According to Beidler the ability of taste receptors to respond to a variety of cations would vary with the nature of the reacting radical and with the proximity of neighboring reacting groups as well. Differences in responses of taste substances as already demonstrated by Beidler, Fishman, 
and Hardiman (1955) are thus explainable in terms of difference in the detailed configuration of reacting receptor molecules in different species.

\section{Olfaction}

The olfactory receptors in insects are only superficially different from the taste receptors (Fig. 18). In vertebrates the difference is of a more fundamental nature (Fig. 19). In mammals olfactory receptors occur more or less evenly distributed in a small patch $\left(2.5 \mathrm{~cm} .^{2}\right)$ of epithelium covering the medial wall of the superior concha and the lateral wall of the nasal septum. In the rabbit there is a moderate density gradient diminishing centrifugally (Allison and Warwick, 1949). Proximal processes of the olfactory receptors synapse in the glomeruli of the bulb (Fig. 20). In the rabbit approximately 26,000 receptors synapse with each glomerulus. There are approximately 1,900 glomeruli which synapse with about 24 mitral and 68 tufted cells. Thus, each glomerulus, a]though connected with other glomerulus systems by short-axon granular cells and collaterals from mitral and tufted cells, receives impulses from a segregated and independent group of olfactory fibers and projects them through a limited number of mitral and tufted cells to the secondary olfactory centers at the base of the telencephalon.

The question of whether there are a few basic types of receptors analogous to the specific taste receptors, or a multitude of receptors or receptor units each sensitive to different chemicals or whether all receptors are sensitive to all chem-

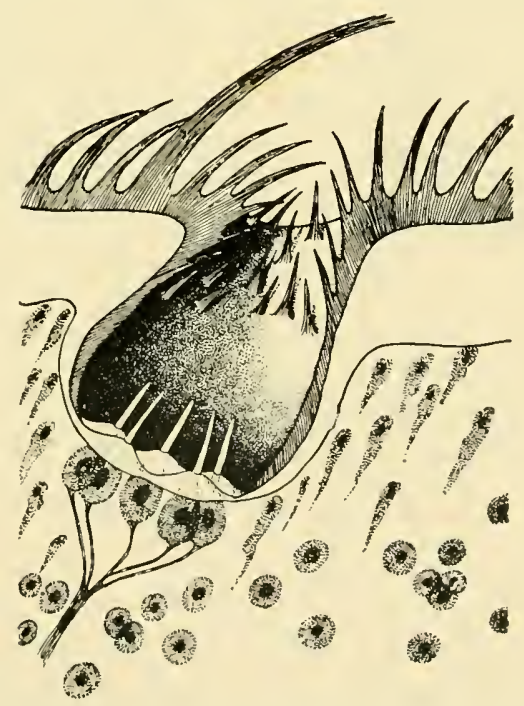

FIG. 18. Olfactory pit on antenna of dipterous insect (Liebermann, 1926) 
icals has not yet been satisfactorily answered. The problem was first approached by studying cross adaptation, odor mixtures, and cases of differential anosmia. Le Gros Clark and Warwick (1946) found that small lesions in the olfactory bulb lead to retrograde atrophy of receptors scattered diffusely over the entire septum. As Allison and Warwick (1949) pointed out, this observation suggests an absence of point-to-point localization of the projection of the olfactory epithelium on the olfactory bulb. The projection is so diffuse that a small area of the epithelium may be represented over a considerable area of the bulb. Each glomerulus probably receives fibers from receptors scattered widey throughout the olfactory epithelium. A glomerular system is at least automatically set up to mediate responses to specific odors.

Cross adaptation and its absence suggests different receptor groups each of which is sensitive to a different group of chemicals, i.e., a receptor mosaic. But, as long as the vapor pressure and hence the concentration of the stimulus is not controlled, the various results obtained could equally well be a result of concentration effects. The subjective sensations elicited by various mixtures tend to bolster the mosaic theory in that trained experts can analyze the constituents of mixtures. In this respect olfaction resembles an auditory analogue. It should be pointed out here, however, that the auditory stimulus is of the same nature throughout the auditory spectrum. By analogy, olfactory stimuli which elicit different odor sensations need not necessarily operate through different mechanisms.

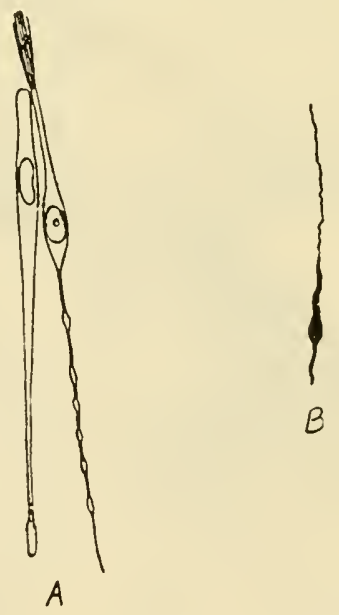

A. Isolated olfactory cell and sustentacular cell from a frog. (After Schultze 1862, Plate 1, Figure 4.)

$B$. A Golgi preparation of an olfactory cell from a pike (Esox), in which not only the cell body and the basal nerve fiber process are well shown but also the long peripheral olfactory flagellum. (After Jagodowski, 1901, Figure 8c.)

FIG. 19. Olfactory receptors from the frog (from Crozier, 1934) 


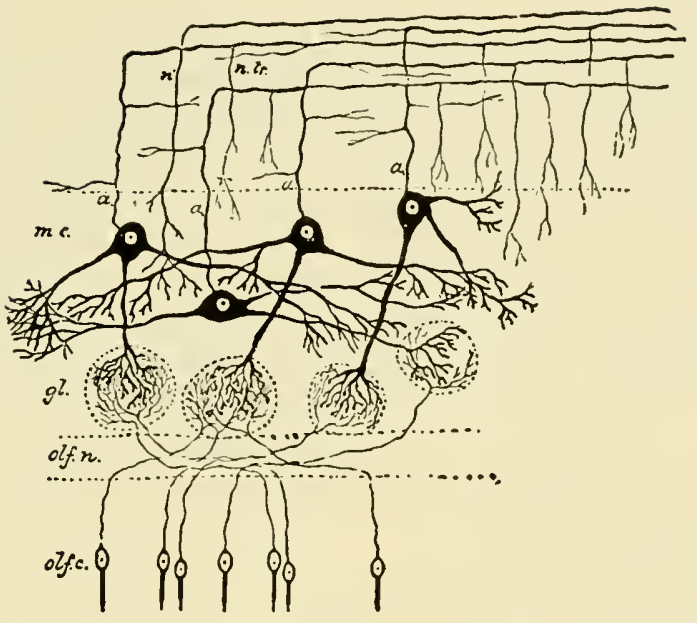

Diagram illustrating the relations of the olfactory nerve fibers to the neurons of the second order in the olfactory bulb. olf.c., olfactory nerve cells in the olfactory epithelium; olf.n., olfactory nerve; mc., mitral cells; gl. olfactory glomeruli; n.tr., olfactory tract. (Schäfer, "Organs of the Senses," Longmans, Green \& Co., Inc.)

Fig. 20. The relation between the olfactory receptors and other neural cells of the olfactory bulb (from McCord and Witheridge, 1949.)

What would appear most helpful at this time would be an accurate identification of the adequate olfactory stimulus; or, to restate the desideratum as a question, is every receptor sensitive to all odors provided that a critical concentration can be applied? As in the case of the sense of taste, an electrical approach to the problem appears to be necessary. However, the only successful recordings which have been reported in the literature thus far were obtained with electrodes located in the olfactory tract or bulb several synapses removed from the receptors. ${ }^{1}$ Upon the interpretation of the pattern of discharges obtained depends the answer to the question of receptor specificity.

${ }^{1}$ Since this symposium was presented three papers have been published, two of which report successful efforts to place electrodes directly on the olfactory receptor fibers. Beidler and Tucker (1955) have succeeded in recording electrically from nerve bundles in isolated opossum nasal epithelium exposed to pure air and odors. Results indicate that one fiber may not respond to a given odor as well as another fiber, although this relationship may be reversed if a different test odor is chosen. Walsh (1955) studied unitary activity in the bulb of the rabbit by means of micropipette electrodes. Cells of one type detected by this method responded markedly to stimulation by butyl acetate but failed to respond to high concentrations of acetophenone or tertiary amyl alcohol. Schneider (1955) has recorded impulses from single fibers of olfactory receptors in the antennae of male moths being stimulated with the odorous material produced by the sex attractant glands of the female. These results are preliminary, and no information relative to the specificity of the different receptors was given. 
Adrian (1950), recording from olfactory tract fibers in the rabbit, was able to demonstrate that there are large scale differences in the level and evolution of excitation in different parts of the olfactory epithelium. However, odors of any class could stimulate any area provided the concentration was sufficiently high. He thought it was probable that olfactory analysis is brought about by the effect of the general arrangement of the olfactory organ on stimulus accessibility and strength rather than by differential sensitivity of receptors in different parts of the organ. Nevertheless, owing to regional analysis, whether resulting from differential receptor sensitivity or differences imposed upon the stimulus by the structure of the organ, different smells set up different patterns of excitation. No indication was found that the olfactory epithelium is a uniform mosaic of receptors each with specific affinities for certain kinds of smells. Yet the entire olfactory organ seems to act as an analyzer for molecules of different physical and chemical properties.

The evidence given above is the best to date that there may be a number of functionally different types of olfactory receptors. It should be mentioned before leaving this subject that the highly specific reactions of certain organisms to

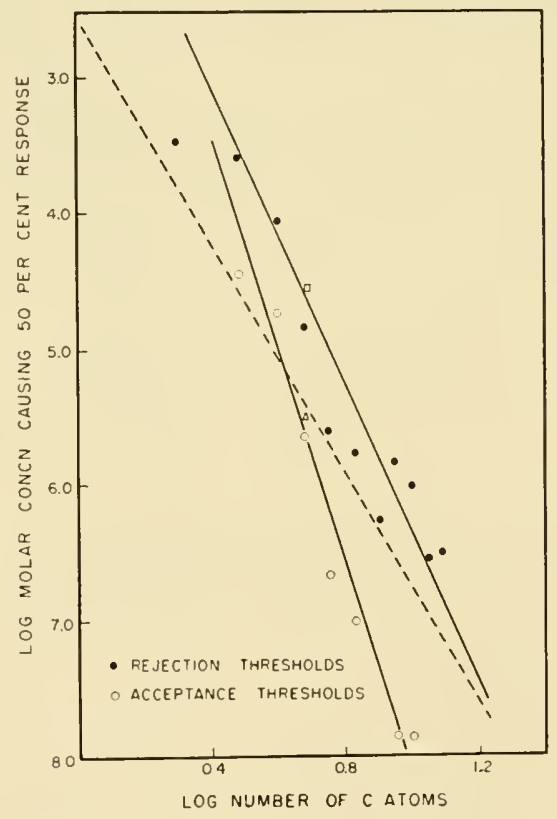

Fig. 21. Relation of olfactory thresholds (acceptance and rejection) for aldehydes to chain length. The broken line represents the relation for alcohols based upon the data of Dethier and Yost (1952). The square and triangle represent rejection and acceptance thresholds, respectively, for iso-valderaldehyde. Acceptance thresholds are based upon maximum response. (Dethier, 1954a). 
restricted olfactory stimuli by no means indicates a high receptor specificity. While it is tempting to think that different species may be highly specialized at the receptor level in this respect, possibly, for example, in a manner analogous to protein and antigen/antibody specificity, it is equally likely that the specificity is at a much higher level corresponding to Tinbergen's (1951) "innate releasing mechanisms."

At the moment there is no evidence from insects either that there are different receptor types or that the receptors are restricted in their ranges of sensitivity. Thus, for example, caterpillars (with no more than six to ten receptors) can respond to a wide variety of odors (Dethier, 1941). A similar situation exists with respect to the water beetle, Laccophilus (Hodgson, 1951). Furthermore, extirpation of as many as $90 \%$ of the olfactory receptors as in the case of ablation of antennal segments of honeybees (von Frisch, 1919) and dung bettles (Dethier, 1947) does not seem to hamper these insects in their ability to operate normally in their highly olfactory world other than by raising threshold.

It appears to be quite pointless at this time to theorize on the nature of the transducing mechanism on the basis of correlations of subjective modalities of odors with physico-chemical properties of the stimulating compounds. At any rate the approach has not been profitable thus far. On the other hand, a study of the relation between stimulating efficiency (threshold) and molecular characteristics does indeed seem to hold some promise. Ideally, as with taste, one

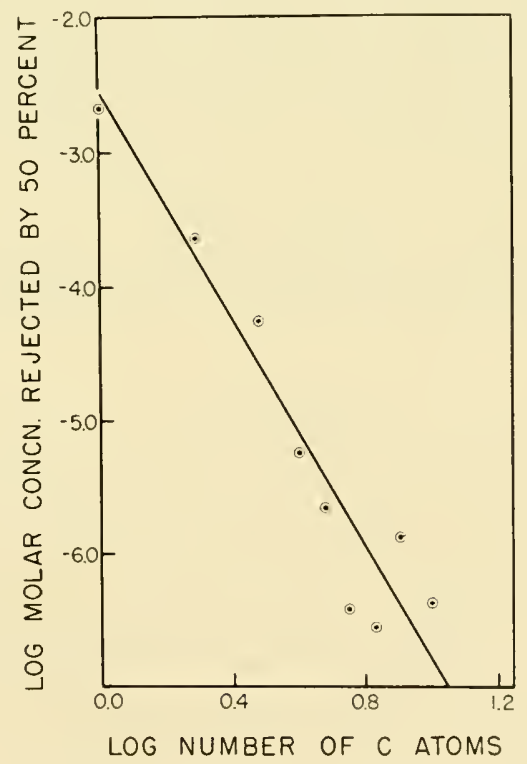

FIG. 22. Relation of olfactory thresholds (rejection) of alcohols to chain length (Dethier and Yost, 1952). 


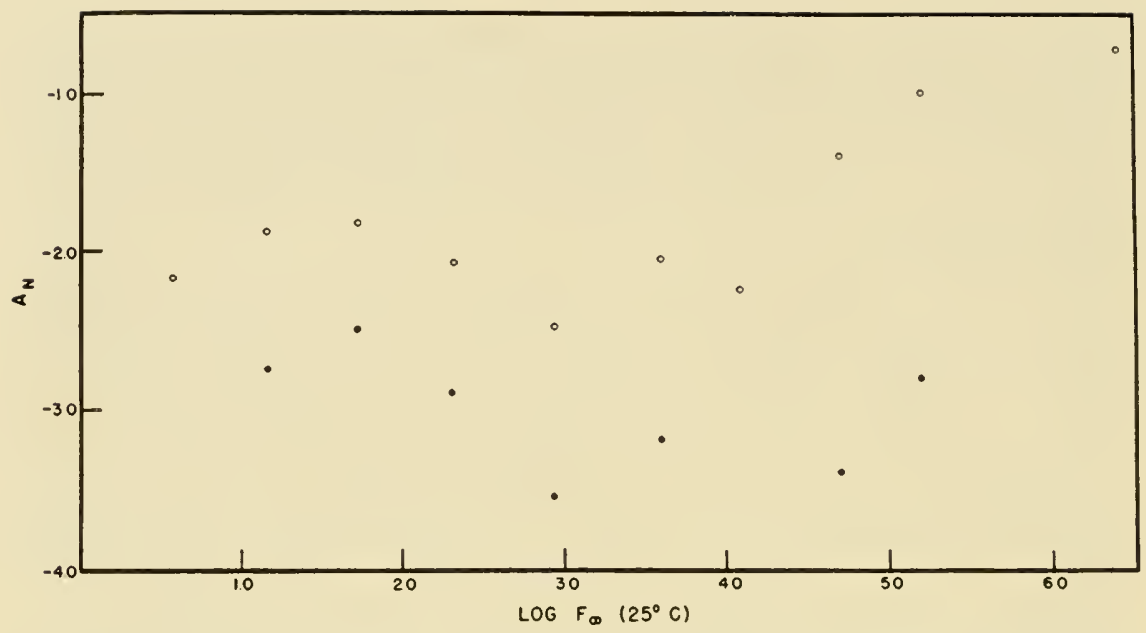

FIG. 23. Comparison in terms of thermodynamic activity of the stimulating effectiveness of homologous aliphatic aldehydes on the olfactory receptors of the blowfly. The open circles represent rejection thresholds and the solid circles, acceptance thresholds.

should be able to work with single receptor units. But, as with taste, preliminary studies of populations of receptors are of introductory value. On the basis of measurements made with insects by Dethier and Yost (1952) and Dethier (1954a) and with man by Mullins (1955a) it is clear that the olfactory thresholds stated as thermodynamic activities $(p t / p s)$ for members of certain homologous series, notably alcohols and aldehydes, are nearly constant over the middle range of chain length (Figs. 10, 21, 22, 23). On the other hand, recent measurements of fatty and olfactory thresholds in the dog (Neuhaus, 1953) and in the honeybee (Schwarz, 1955) do not exhibit such a clean-cut relationship when expressed in activity units. When expressed as numbers of molecules per cc, of air at threshold both series show an increase in sensitivity for $\mathrm{C}_{2}$ to $\mathrm{C}_{4}$ (although the difference is very small in the bee) and a decrease from $C_{4}$ to $C_{8}$. Dethier (1954b) pointed out that the relationship observed with the alcohols suggests that the phenomenon under investigation may involve the establishment of an equilibrium and as such represent a physical rather than a chemical process involving specific receptor substances. Mullins (1954) has analyzed the data of Dethier and Yost (1952) by plotting the product of the thermodynamic activity at threshold and the molal volume of the compounds against chain length and assigning a calculated membrane solubility parameter of 11.5 . The analysis suggests that the vapor of the first three alcohols may not be in equilibrium with the organism. ${ }^{2}$

${ }^{2}$ In a recent paper Mullins (1955b) has analyzed existing data and presented additional data which indicate that olfaction is not at all similar to an equilibrium 
Mullins (1955a), on the basis of threshold measurements in man of $n$-paraffins, $n$-alcohols, and $n$-alkyl chlorides, in which at any given number of carbon atoms the thresholds are about the same for any series, has shown that thresholds $(p t, p s)$ are lowest for the $4-5$ carbon members and rise for longer and shorter membered chains. On the basis of these and other more extensive data he has proposed that two molecular parameters are of importance in olfactory stimulation: (1) molecular shape and (2) cohesive energy density (the energy of vaporization of a liquid per unit volume). This second factor is roughly related to the oil 'water partition coefficient (for full discussion consult Mullins, 1954).

Obviously only a beginning has been made in analyzing the process of olfaction by critical experiment, but even at this early stage indications are emerging which suggest that the process is less likely to be a chemical than a physical one.

\section{Conclusion}

The attempts to analyze the mechanism of chemoreception at the molecular level at which the receptor is conceived of as a transducer are of relatively recent origin. Obviously they have not progressed far enough to supply us with satisfactory answers to the questions which opened this discussion. Yet they have supplied us with some broad and very suggestive hints. In the light of our present knowledge it is highly probable that the process of olfaction and the process of taste are more nearly biophysical than biochemical reactions. The case of sugars may be a special one which involves a more specific type of reaction. That the sugars might be exceptional is not unexpected in view of their special and important role in carbohydrate metabolism in many tissues of the body.

\section{Acknowledgment}

The records in Figure 15 were obtained by M. Wolbarsht on equipment made available through the courtesy of the United States Naval Medical Research Institute.

\section{References}

Adrian, E. D. 1950. Sensory discrimination with some recent evidence from the olfactory organ. Brit. Med. Bull. 6: 330-333.

Allison, A. C. and R. T. T. Warwick. 1949. Quantitative observations on the olfactory system of the rabbit. Brain 72: 186-197.

Andersson, B., S. Landgren, L. Olsson and Y. Zotterman. 1950. The sweet taste fibres of the dog. Acta physiol. scand. 21: 105-119.

process. Adaptation after olfactory stimulation may be considered to be an equilibrium effect. Evidence is presented to support the suggestion that the excitatory action of molecules is by virtue of their ability to produce a local disorder in the oriented molecular structure of the cell membrane. 
Andersson, B. and Y. Zotterman. 1950. The water taste in the frog. Acta physiol. scand. 20: 95-100.

Beidler, L. M. 1952. Our taste receptors. Sci. Monthly 75: 343-349.

Beidler, L. M. 1953. Properties of chemoreceptors of tongue of rat. J. Neurophysiol. 16: $595-607$.

Beidler, L. M. 1954. A theory of taste stimulation. J. Gen. Physiol. 38: 133-139.

Beidler, L. M., I. Y. Fishman and C. W. Hardiman. 1955. Species differences in taste responses. Am. J. Physiol. 181: 235-239.

Beidler, L. M. and D. Tucker. 1955. Response of nasal epithelium to odor stimulation. Science 122: 76.

Brink, F. and J. M. Posternak. 1948. Thermodynamic analysis of the relative effectiveness of narcotics. J. Cell. \& Comp. Physiol. 32: 211-233.

Chadwick, L. E. and V. G. Dethier. 1947. The relationship between chemical structure and the response of blowflies to tarsal stimulation by aliphatic acids. J. Gen. Physiol. 30: 255-262.

Chadwick, L. E. and V. G. Dethier. 1949. Stimulation of tarsal receptors of the blowfly by aliphatic aldehydes and ketones. J. Gen. Physiol. 32: 445-452.

Cohen, M. J., S. Hagiwara and Y. Zotterman. 1955. The response spectrum of taste fibres in the cat: a single fibre analysis. Acta Physiol. Scand. 33: 316-332.

Crozier, W. J. 1934. Chemoreception. In "A Handb. of Genl. Expt. Psychol", ed. C. Murchison. 987-1036. Worcester, Mass. Clark Univ. Press.

Dethier, V. G. 1941. The function of the antennal receptors in lepidopterous larvae. Biol. Bull. $80: 403-414$.

Dethier, V. G. 1947. The role of the antennae in the orientation of carrion beetles to odor. J. New York Ent. Soc. 55: 285-293.

Dethier, V. G. 1951a. The limiting mechanism in tarsal chemoreception. J. Gen. Physiol. 35: 55-65.

Dethier, V. G. 1951b. Taste sensitivity to compounds of homologous series. Am. J. Physiol. 165: 247-250.

Dethier, V. G. 1952. Taste sensitivity to homologous alcohols in oil. Fed. Proc. 11: 34.

Dethier, V. G. 1953. Chemoreception. In "Insect Physiology", ed. K. D. Roeder. pp. 544-576. New York. John Wiley \& Sons.

Dethier, V. G. 195ta. Olfactory responses of blowflies to aliphatic aldehydes. J. Gen. Physiol. 37: 743-751.

Dethier, V. G. 1954b. The physiology of olfaction in insects. Ann. N. Y. Acad. Sci. 58: 139-157.

Dethier, V. G. 1955. The physiology and histology of the contact chemoreceptors of the blowtly. Quart. Rev. Biol. 30: 348-371.

Dethier, V. G. and L. E. Chadwick. 1948a. Chemoreception in insects. Physiol. Rev. 28: 220-254.

Dethier, V. G. and L. E. Chadwick. 1948b. The stimulating effect of glycols and their polymers on the tarsal receptors of blowflies. J. Gen. Physiol. 32: 139-151.

I)thier, V. G. and I. E. Chadwick. 1950. An analysis of the relationship between solubility and stimulating effect in tarsal chemoreception. J. Gen. Physiol. 33: 589-599.

Dethier, Y. G. and M. T. Yost. 1952. Olfactory stimulation of blowflies by homologous alcohols. J. Gen. Physiol. 35: 823-839.

Ferguson, J. 1951. Relations between thermodynamic indices of narcotic potency and the molecular structure of narcotics. In "Mecanisme de la Narcose". Coll. Internat. Centre Nat. Rech. Sci. 26: 25-39. 
Frings, H. 1946. Gustatory thresholds for sucrose and electrolytes for the cockroach, Periplancta americana (Linn.). J. Exp. Zool. 102: 23-50.

Frings, H. 1948. A contribution to the comparative physiology of contact chemoreception. J. Comp. \& Physiol. Psychol. +1: 25-3t.

Frings, H. and B. L. Cox. 1954. The effects of temperature on the sucrose thresholds of the tarsal chemoreceptors of the flesh fly, Sarcophaga bullata. Biol. Bull. 107: $360-36.3$.

Frings, H. and B. R. O'Neal. 1946. The loci and thresholds of contact chemoreceptors in females of the horsefly, Tabamus sulcifrons Macq. J. Exp. Zool. 103: 61-79.

von Frisch, K. 1919. Über den Geruchsinn der Biene und seine blutenbiologische Bedeutung. Zool. Jahrb., Abt. f. Allg. Zool. u. Physiol. 37: 1-238.

von Frisch, K. 1935. Über den Geschmackssinn der Biene. Zeit. f. Vergl. Phỹsiol. 21: $1-156$.

Gavaudan, P. 1951. La pharmacodynamie compáree des toxiques physiques. In "Mecanisme de la Narcose", Coll. Internat. Centre Nat. Rech. Sci. 26: 53-75.

Hassett, C. C., I. G. Dethier and J. Gans. 1950. A comparison of nutritive values and taste thresholds of carbohydrates for the blowfy. Biol. Bull. 99: 446-453.

Hodgson, E. S. 1951. Reaction thresholds of an aquatic beetle, Laccophilus maculosus Germ. to salts and alcohols. Physiol. Zool. 27: 131-140.

Hodgson, E. S., J. Y. Lettvin and K. D. Roeler. 1955. The physiology of a primary chemoreceptor unit. Science 122: $\$ 17-418$.

Horlgson, E. S. and K. D. Roeder. 1956. Electrophysiological studies of arthropod chemoreception. I. General properties of the labellar chemoreceptors of Iiptera. J. Cell. Comp. Physiol. (in press)

Le Gros Clark, IT. E. and R. T. Warwick. 1946. The pattern of olfactory innervation. J. Neurol. Neurosur. Psychiat. 9: 101-111.

Liebermann, A. 1926. Correlation zwischen den antennalen Geruchorganen und der Biologie der Musciden. Z. Morphol. Ökol. Tiere 5: 1-97.

McCord, C. P. and IV. N. Witheridge. 1949. Odors. Physiology and Control. New York. McGraw Hill. 405p.

Mullins, L. J. 1954. Some physical mechanisms in narcosis. Chem. Revs. 5t: 289-323.

Mullins, L. J. 1955a. Olfactory thresholds for some homologous series of compounds. Fed. Proc. 1t: 105-106.

Mullins L. J. 1955b. Olfaction. Ann. N. Y. Acad. Sci. 62: 247-276.

Neuhaus, IV. 1953. Über die Riechscärfe des Hundes für Fettsäuren. Zeit. Vergl. Physiol. 35: 527-552.

Pfaffmann, C. 1941. Gustatory afferent impulses. J. Cell. Comp. Physiol. 17: 243-258.

Pfaffmann, C. 1951. Taste and smell. In "Handb. Exp. Psychol.". S. S. Stevens, ed. pp. 1143-1171. New York. John Wiley \& Sons. 1436p.

Poussel, H. 1951. L'excitation indifférente et les conditions énergétiques de son déclenchement. In "Mécanisme de la Narcose". Coll. Internat. Centre Nat. Rech. Sci. 26: 157-162.

Renqvist, Y. 1920. Der Schwellenwert des Geschmackreizes bei einigen homologen und isomeren Verbindungen. Skand. Archiv. f. Physiol. 40: 117-124.

Schneider, D. 1955. Mikro-elektroden Registrieren die elektrischen Impulse einzelner Sinnesnervenzellen der Schmetterlingsantenne. Industrie-Elektronik 3/4: 3-7.

Schwarz, R. 1955. Über die Riechschärfe der Honigbiene. Zeit. f. Vergl. Phỵsiol. 37: $18-21$.

Tinbergen, N. 1951. The Study of Instinct. Oxford. Clarendon Press. 228p.

Walsh, R. R. 1955. Unitary activity in the olfactory bulb of the rabbit. Fed. Proc. 14: 157 . 
Zotterman, Y. 1949. The response of the frog's taste fibers to the application of pure water. Acta Physiol. Scand. 18: 181-189.

Zotterman, Y. 1950. The water taste of the frog. Exper. 6: 57-58.

Chatruan GERARD: We are very grateful to you for giving us this good survey of taste sense and smell sense and not too much nonsense.

Dr. Wade H. Marshall (National Institutes of Health): I would like to plead guilty to being a dunce; I missed the third neuron on that fly system.

Dr. DetIIER: You have nothing to plead guilty about because nobody knows what this one does. We have been unable to trace its fiber beyond the base of the hair. This would make it a very fine candidate for mechanical reception as found in other receptors but, unfortunately, it does not conform to this concept and this hair does not respond very well to mechanical stimulation.

There are those of my colleagues who would be happy if this were some sort of a ganglion cell. All I can say is heaven forbid.

Dr. Roscoe Brady (National Institute of Mental Health): On the slide with the increasing chain in fatty acids on the taste rejection, where you get into the four, five and six carbon fatty acids, you immediately come into the stench range and, of course, must rule out the smell with these taste studies.

Dr. DetiIIER: The data which you saw were for insects and it is possible, with these very handy beasts, to work either with taste or with smell without the other interfering because one can remove all of the olfactory receptors and render the animal completely anosmic.

Dr. Manuel Morales (Naval Medical Research Institute): I have two related questions. At two points in your talk you indicated the possibility of some chemical reaction, and I believe you were thinking of the stimulant as being a substrate in this reaction, something that is used up and perhaps, if one washed it away one wouldn't get all of the stuff back. Of course, the second possibility is that the stimulant could be acting as an activator or an inhibitor on the reaction that was using up something else. I wondered if you would, perhaps, say if the other mechanism has been considered.

The second question was: In those cases where you have two substances that are very similar and yet different in their effect-I think you mentioned the $\mathrm{D}$ and $\mathrm{L}$ formula, would it be possible in those cases, if you had a tracer, to decide whether the substance is actually incorporated as a receptor and cannot be washed away or whether it can be washed away? This, of course, bears on deciding between the two possibilities in my first question.

Dr. Dethier: In answer to your first question, we have considered both possibilities that you mention. We have not carried this to the point where we have experimentally any more evidence for one than for the other. I might add that this is very recent work and you are hearing the early stages of it. 
With regard to the use of the tracers, we have also considered the possibility that we are somewhat pessimistic in our outlook, maybe unjustifiably so, because frankly, I do not know very much about the technique involved. I have wondered in my own mind whether it would be possible to get the answer from tracers as to whether it washes away or not. Maybe you can tell us whether in small preparations of this sort, where we are working in micron dimensions, this method would help us in telling whether the stimulating substance is part of the receptor or can be washed away.

Dr. Morales: I do not know anything about tracer technique, either. I would propose this as a logical point because, you see, if you start from the premise that you want a very small amount of something to do a lot then the idea of having this be an activator or inhibitor is much more attractive than to have a substrate. If the thing is incorporated and cannot be washed away, then the chances are that this is really participating and being used up in the reaction.

Dr. Detiner: I agree fully and it is just a question of whether it is technically possible or not. If it is, then, we shall sometime get going on it.

Dr. Alex Rich (National Institute of Mental Health): I was a little puzzled by your statement about the fact that salts could reach a threshold concentration whereby sugars would not elicit a response. Can you do vice versa, or not? Apparently one of these fibers is the fiber which is responding to the salt and one to the sugar; isn't it rather bizarre that the two are in such unbalance?

The second question has to do with the fact that there is no competitive inhibition with sugars. Now, this puts the sugars, as you mentioned, in a class quite by themselves. Does this literally mean that if you have a large amount of sugar which has no response at all and put it into a solution with a small amount of sugar which has a response that you will still get a response? Is it that type of experiment?

Dr. Detiner: If I may answer your second question first; when we put a small amount of the stimulating sugar with a large amount of the non-stimulating sugar, the only effect we get is one which can be satisfactorily accounted for on a dilution basis.

DR. RicH: You mean the response is the same as the non-responding sugar, perhaps?

Dr. Dethier: Yes, unless the small amount of stimulating sugar which is incorporated is present in threshold quantities. We have been unable to demonstrate any competitive inhibitions.

Now in answer to your first question, one can prevent the salt response by adding sugar and the sugar response by adding salt. However, a concentration of salt can be reached beyond which no amount of sugar can stop it. In other words, when one is working with maxima of the two available compounds the salt takes over. Now, this is not as strange as it may seem, because we are 
dealing with two receptors and, to my way of thinking, the addition is taking place in the central nervous system. In those vertebrates which have been studied by electrical methods it has been shown that salt and sugar do not interfere with each other at the peripheral level but at the central level. The business of sweetening a sour drink by adding a lot of sugar to it seems undoubtedly to be a central nervous system phenomenon.

Cilairmax GeraRd: Let me toss in something. These two receptors, the two hairs, are they specific, one for salt and one for sugar? Or can each one taste either one? How does the central nervous system know which is being exposed to what?

DR. DethiER: There are two fibers in each hair-one fiber for sugar and one for the other.

Chairman Gerard: This is clear.

Dr. Sidney A. Bernhard (Naval Medical Research Institute): I would like to question your enzymatic or activator or inhibitor hypothesis from your data on the sugars. If first you examine the data on the salts alone that could equally well be explained just on the basis of binding in itself. That is, all the data indicate that you have an electrostatic binder phenomenon with the salt. Let us assume that with the sugars all one had was a sort of V'an der IVaals binding, then what one would expect would be that sugars with nearly complementary structures to the site would produce, or would give a positive sensation; whereas, sugars without the complementary site would not. Therefore, you could never find an inhibitor under such a model. Everything which we call a competitive inhibitor would, in fact, taste sweet.

Dr. Detiner: Yes, I see your point. I think that the only answer would be more experiments.

DR. Rich: Apropos of tests and apropos of these experiments, supposing you took a very strongly labeled sugar, both an active one and an inactive one, and put it on the taste bud, then washed it away, and then tested how much of it was bound. It may be that the amount of sugar bound to the receptor sites will be below the threshold of what you can detect with the tracers, but, if you could detect it and you got a difference, then you could account for this particular phenomenon.

DR. DEthiER: I think that the crucial thing is whether there would be enough to detect. I would simply like to say, with regard to the sugar problem, that aside from preparations in mammals where it may be possible to have a single sugar receptor to play with, and I emphasize the "may"-in all other preparations we have to deal simultaneously with a receptor which is opposing this in the central nervous system. If we put in, or attempt to put in inhibitors, even if we did get an inhibited reaction we would never know whether the inhibition was peripheral or whether it was in the central nervous system. So that the most informative studies have been those which relate structure and 
characteristics of the carbohydrates with their stimulating effect because, in this case, you are not touching the other receptor at all.

We have studied 72 sugars and substituted sugars altogether and the degree to which the molecule can be tampered with is incredibly small. So that, just for the carbohydrates, there seems to be a very high degree of specificity which can be correlated with such things as to whether it has an alpha glucoside linkage or a beta glucoside linkage and things of that sort present. All of the compounds which we know act on other receptors and there is no inclination of any limitations imposed by molecular size or shape or disorder of magnitude. 


\title{
Visual Receptors as Biological Transducers
}

\author{
E. F. MacNichol, Jr.
}

The Thomas C. Jenkins Department of Biophysics, The Johns Hopkins Universily, Ballimore, Maryland

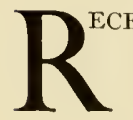

CEPTOR ORGANS may be considered to be transducers whereby particular forms of energy coming from the environment are, to use conamplified, compressed, and encoded in a form suitable for transmission to the central nervous system.

The function of filtering seems to be largely determined by accessory structures such as the mechanical and hydraulic linkages in the ear and the dioptric system of the eye. Additional filtering is undoubtedly done in the receptor cells themselves as, for example, by the specific absorption of a narrow region of electromagnetic spectrum by the visual pigments. The photoreceptors are literally tuned to a narrow band of wavelength occupying about an octave of electromagnetic spectrum.

The biochemistry of the pigments responsible for the highly selective absorption of energy has been worked out in detail, especially by Wald and his colleagues.

How the energy thus absorbed is amplified and encoded into the nerve message is much less well understood. Since the pioneering work of Adrian (1935) and Bronk many receptor physiologists have been engaged in a form of wire tapping which has, fortunately, not yet brought us to the attention of congressional committees. It has been possible to intercept the messages in single fibers of sensory nerves by electrical means so that they can be listened to and recorded oscillographically. Thus the codes produced by many kinds of receptors have largely been broken.

Much of the work that has been done in this field has been admirably reviewed by Granit in his recent book (1955). Study of this material leads to the conclusion that in coded messages are basically the same for all receptors so far studied. This is not surprising since the information must in all cases be in a form suitable for transmission by nerve fibers. The well known "all or nothing" properties of nerve fibers require that the messages be transmitted in a form known in the communications field as pulse time or pulse frequency modulation. The only variations that are allowable are time relationships between impulses or groups of impulses, the frequencies of trains of impulses, or 
the number of impulses in a group. Amplitude variations are effectively removed by the "all or nothing" mechanism.

One of the basic problems in receptor physiology is to determine how, in a given receptor, the energy released by the initial mechanical, chemical or thermal event is amplified and converted into the kind of information carried by nerves.

In the case of the photoreceptors, beyond the initial photochemical process, the mechanism of transduction is largely unknown; whereas, the mechanism by which nerve impulses are propagated is getting to be understood. The biochemists working forward from the initial chemical events in the receptor and the electrophysiologists working backwards from the propagated impulses in the attached nerve fiber are trying hard to close the gap. Being an electrophysiologist, I am going to tell you how far backward Hartline', Wagner2, and myself have gotten on one particular receptor ${ }^{3}$. Let us start with the nerve impulse.

Fig. 1 is a copy of one of Hartline's old records which has been reproduced in the literature many times. It shows the coded message from a single optic nerve fiber of the eye of the horseshoe crab, Limulus. The black band at the lower part of each trace indicates the duration of the stimulating light and the time marks at the bottom of each trace indicate fifths of a second. Each record was taken with a light intensity a factor of ten greater than the one immediately underneath it. It is evident that there is a latent period during which no response occurs, an initial burst of impulses signaling a change in illumination, a maintained discharge which is a function of the intensity of illumination and a cessation of the discharge a short time after the stimulus is removed.

Analysis of similar records shows an approximately logarithmic relationship between the intensity of the light and the number of impulses discharged in a given time interval. This is just an expression of the familiar Weber-Fechner law of psychology. The important point is that the logarithmic relation is determined by the receptor itself and not further along in the central nervous system. An engineer would refer to this logarithmic response as volume compression which is often used to prevent overloading of a communication channel, and, indeed, probably serves that purpose here.

Hartline and his associates have studied many of the properties of the Limulus photoreceptor by measuring the output under a wide variety of input conditions and though the response is too complicated and too variable to permit the deriving of an exact mathematical transfer function for the system, as

${ }^{1}$ H. K. Hartline, Rockefeller Institute for Medical Research, New York.

${ }^{2}$ H. G. Wagner, Commander M. C., USN.

${ }^{3}$ The author assumes full responsibility for the views herein expressed since, due to the pressure of time, his colleagues have not had the opportunity of editing his remarks. 


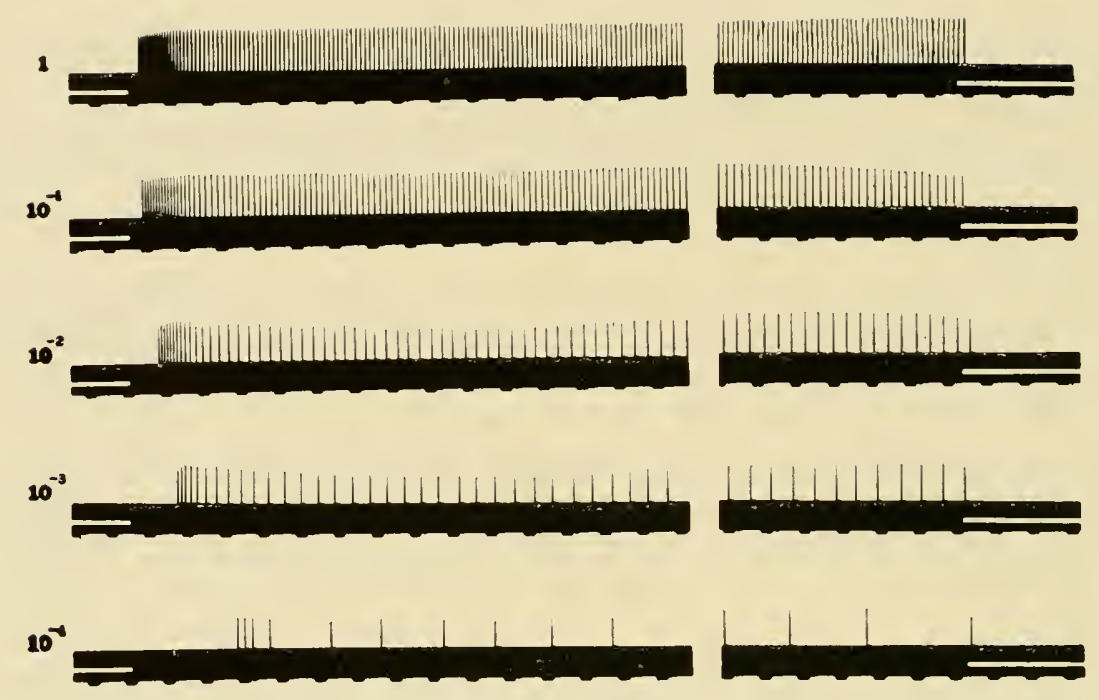

FIG. 1. Response of single Limulus photoreceptor unit to prolonged illumination. The black band above the bottom line of each record indicates the duration of illumination. Time marks are fifths of a second. The intensity of illumination for each record is 10 times that for the one immediately underneath. (Figure courtesy of H. K. Hartline.)

an engineer would do with a photo-cell circuit or a microphone, many qualitative relationships have been established (Hartline et al. 1952). These show what the receptor must do but give no indication of how it does it.

More recently we have tried to get inside the "black box" and learn something about the mechanism. By penetrating the sensory structures with micropipette electrodes we have been able to follow changes in the electrical polarization of the receptor cells themselves. ${ }^{4}$

Starting with the work of Graham and Gerard (1946) and Gerard's associates, many workers have successfully used the micropipette technique to study cell potentials. Wagner and I merely followed their lead and concocted an amplifier which combines a rapid response with extremely low grid current (MacNichol and Wagner, 1954). Then, using saline filled pipettes of less than half micron external tip diameter, we were able to lead off potentials from the interior of the receptor cells with little apparent damage either to the cells or to the potentials. Fig. 2 is a simultaneous recording of potentials obtained from a micropipette electrode and from a pair of electrodes placed on the nerve fibers coming from the sensory structure. Except for the time delay required for the

${ }^{4}$ This work was supported in its initial phases by contract Nonr-248(11) between the Johns Hopkins University and the Office of Naval Research. It is currently being supported by Grant G-922 to the Johns Hopkins University from the National Science Foundation. 


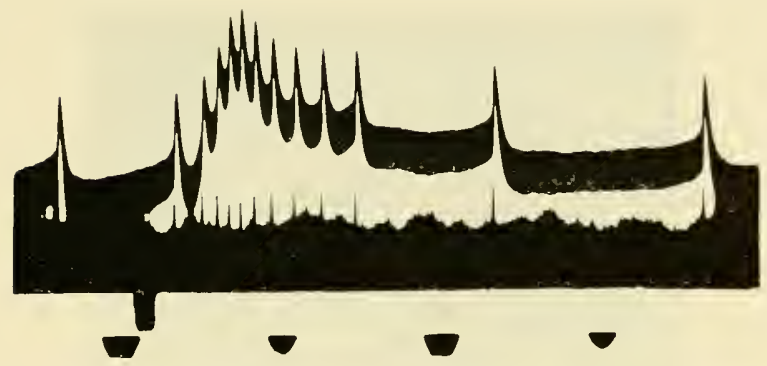

Fig. 2. Simultaneous recording of potentials recorded from a micropipette electrode inserted into a Limulus photoreceptor cell (upper trace) and from the attached nerve bundle (lower trace). The black mark below the lower trace indicates the duration of the stimulating illumination $(.02 \mathrm{sec}$.). Time marks indicate fifths of a second. Upward deflection of the top trace indicates positivity of the micropipette. (From Hartline et al, 1952).

impulses to pass from the receptor along the nerve to the recording electrodes, it is evident that the responses are synchronous. Before discussing the electrical properties of the eye further, let us take a brief look at the histology since it is important in interpreting the results.

The lateral eyes of Limulus are of the compound variety like those of flies but many times larger. They contain less than 1000 sensory elements or ommatidia which is far less than the number in the fly's eye. The sensory elements are also very much larger. Fig. 3 is a photograph of a stained section through one of the eyes. ${ }^{5}$ The original was colored with Mallory's stain. The light colored carrot shaped objects are transparent in life and are made of a crystalline material which acts as the optical system of the eye and conducts the light into the bundles of cells at the base which are the retinulae, or receptor units. Coming from these are nerve fibers which converge to form the optic nerve.

A schematic section of an ommatidium is shown in Fig. 4 .

There are a number of large elongated cells known as the retinula cells, shaped and grouped like the segments of an orange. Through the center of this structure runs a central canal containing the distal process of a large spherical cell known as the eccentric cell. The eccentric cell has a large axon but the retinula cells also give rise to smaller axons. Fig. 5 is a drawing of a transverse section through an ommatidium showing the grouping of the retinula cells about the central canal and the distal process of the eccentric cell passing through it.

In our preparation the excised eye was mounted in a stainless steel clip and sectioned perpendicular to the surface of the cornea with a razor blade. The upper portion of the eye was discarded and the lower portion held in the clip transferred to a chamber filled with physiological solution. The appearance of the cut surface of the eye, as viewed through a dissecting microscope, is very

${ }^{5}$ Histological photographs courtesy of William Miller, Rockefeller Institute. 


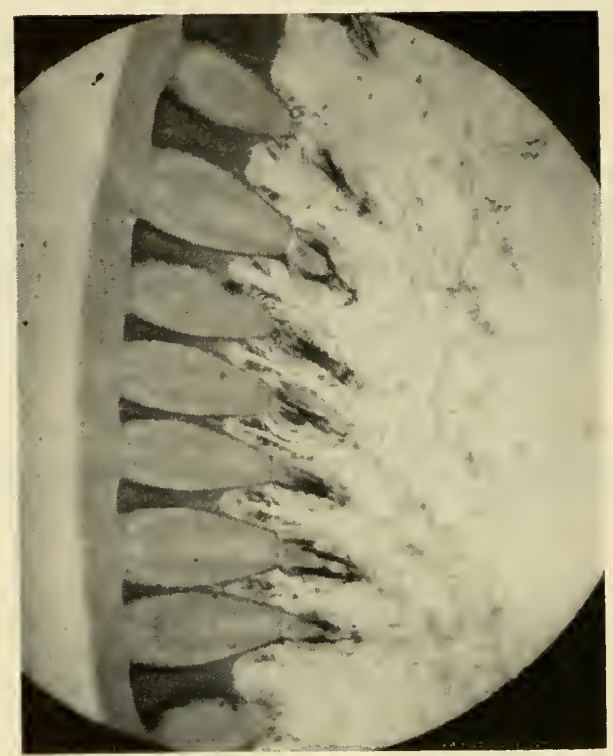

FIG. 3. Stained section through Limulus eve showing seven ommatidia. Sections moderately bleached and stained with Lee Brown modification of Mallory's aniline blue stain. (Courtesy of W. H. Miller).

much as shown in Fig. 6 except that the nerve bundles do not show up as well as in the slide which was silver stained. The black clumps at the left are the ommatidia, the nerve fibers come out toward the right and disappear where the section passes through a part of the nerve fibers. They then reappear at the extreme right of the figure where they converge to form the optic nerve. Lateral connections between the nerve fibers are also apparent. They appear to subserve an inhibitory effect which will be discussed later.

Due to the pigment in their capsules the interior of the ommatidia cannot be seen, though some of the details of the structure can be seen in an occasional unit which has been sliced open by the cut.

A chlorided silver reference electrode was placed in the solution and a micropipette held in a saline filled chlorided silver tube lowered over the ommatidia. The electrodes were connected through our special input circuit and a D.C. amplifier to an oscillograph and loud speaker. Usually the puncture of an ommatidium caused a large steady deflection of the oscilloscope trace, about 50 millivolts, the pipette being negative, but there was no response to illumination. As a pipette was thrust further inward in small increments the potential would abruptly go back to zero, then negative again and then back to zero again, several times. The negative deflections were taken to be the resting potentials of the retinula cells which occupy most of the volume of the ommatidium. As 


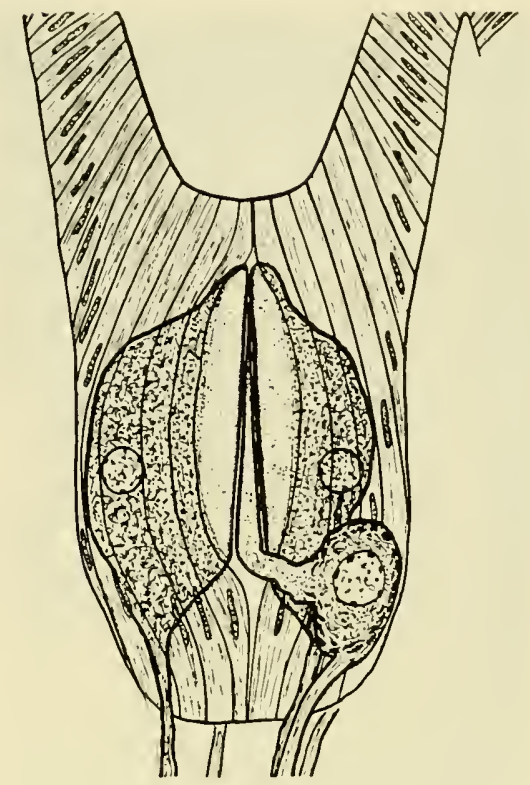

FIG. 4. Schematic drawing of sagital section through Limulus ommatidium. (From Demoll, R., 1917, Die Sinnesorgane der Arthropoden ihr Bau und ihre Functionen. Friederick Vieweg \& Sohn. Braunschweig).

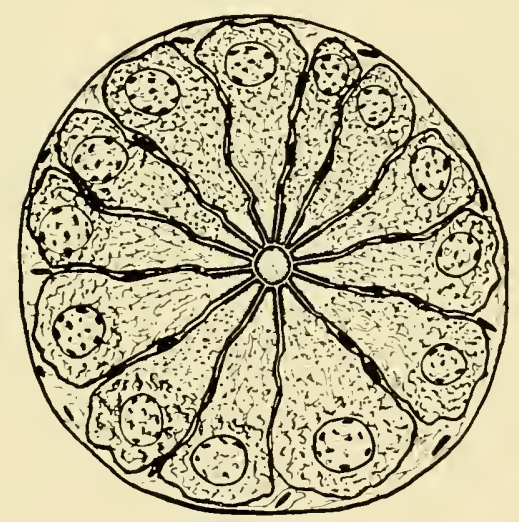

Fig. 5. Schematic drawing of transverse section through Limulus eye. (Same source as Fig. 4.)

the pipette was advanced the response to light was tested after each thrust. After persistent probing a response to light was usually found unless the ommatidium had been damaged, but always in a highly localized region of the ommatidium. If the pipette was moved just a little bit, the response was lost. 


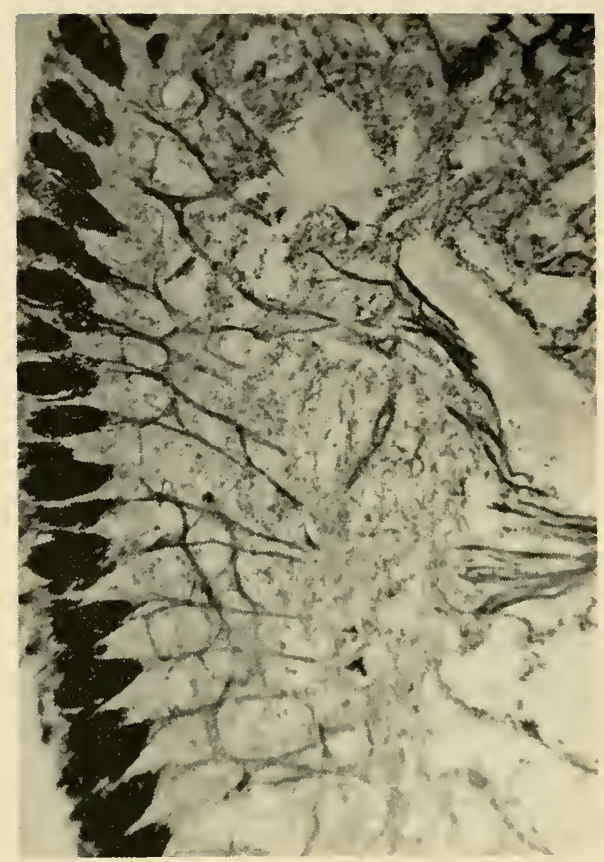

Fig. 6. Section through Limulus eye showing cross connections between the nerve fibers (silver stained). (Courtesy of II. H. Miller.)

Since the retinula cells occupy most of the space within an ommatidium the electrode should have passed through at least one every time the pipette was thrust into an ommatidium so we do not think they give rise to any electrical activity in response to light. The eccentric cell, on the other hand, occupies a very small portion of the total volume and is the only other histologically identifiable structure within the ommatidium.

We think that it is necessary to obtain some contact with an eccentric cell to obtain activity. This view has been confirmed by direct visual observation. On several occasions we have been able to see the cell bodies of intact eccentric cells in ommatidia that have been torn open when the eyes were sliced. It was possible to penetrate these exposed cells under direct visual control and we obtained large spikes in response to light when the electrode was thrust into them. We have never obtained any sort of electrical activity, other than a resting potential, from the exposed retinula cells. Therefore, we are tempted to indict the eccentric cell as the structure in which the spikes originate. However, the situation is more complicated.

Three types of activity in response to light have regularly been found in ommatidia as shown in Fig. 7 . 

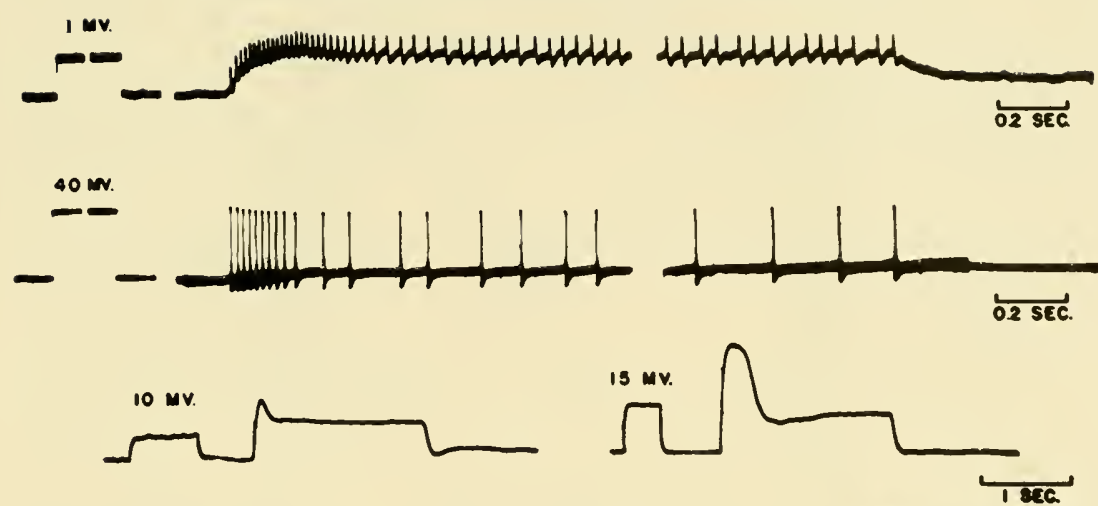

Fig. 7. Typical responses from micropipettes inserted into Limulus ommatidia. (Upward deflection indicates that the pipette became more positive.)

Sometimes we obtained very large spikes like those from visible eccentric cells as shown in the middle trace. On other occasions large slow potentials like those shown in the bottom two traces were obtainerl. Very frequently small spikes superimposed on small slow potentials were observed as shown on the top line. Which type of response was obtained seems to have depended upon the location of the electrode tip. We have never seen large spikes and large slow potentials together. ${ }^{6}$ There have as yet been no positive identifications of the origin of the slow potentials, unless we assume by elimination of other structures that they arise in the distal process of the eccentric cell.

When we obtained both slow potentials and spike activity, we believed that we were recording from a location in which the electrode intercepted currents leaking from the separate sites of origin of the slow and spike potentials.

We have investigated the quantitative relationships between light intensity, the slow potentials and the spike frequency in preparations in which we were able to get both potentials simultaneously (MacNichol et al., 1953). The top record of Fig. 7 is one of the series used in this investigation. We were able to measure the amplitude of the slow potential on the record and to measure the frequency of discharge. The slow potentials were positive, indicating a depolarization of the receptor cells and the frequency of the spikes appears to be a function of both the amplitude of the depolarization and of its rate of change. Notice that the spikes were much faster in the beginning as the slow potential rose. For simplicity we have thus far studied only the steady state conditions during the third second of illumination when transient effects had subsided.

Fig. 8 shows the relationship between the slow potential and the logarithm

${ }^{6}$ An alternative hypothesis is that the large slow potential is an injury effect in which a spike is initiated but the cell cannot recorer its resting potential until the light has been turned off. 


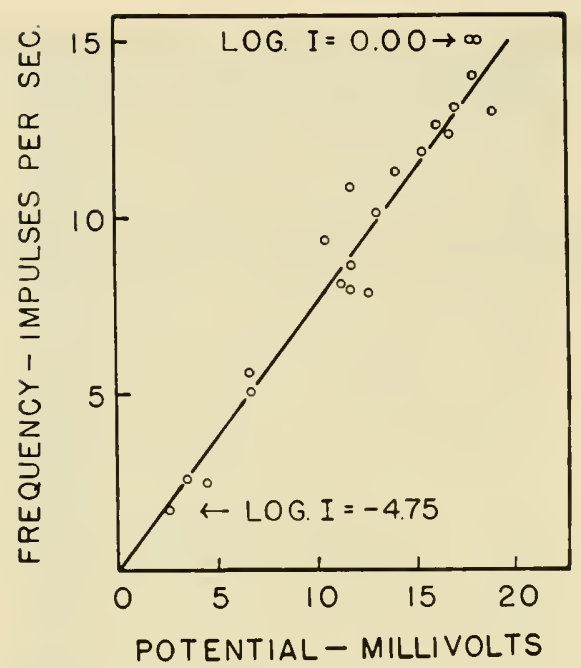

FIG. 8. The relationship between the frequency of discharge and the potential change within a Limulus photoreceptor cell in response to prolonged illumination. After dark adaptation records were taken with progressively increasing illumination (0.25 log. unit steps). Sufficient time was allowed between records to prevent light adaptation. The number of impulses during the third second of illumination was plotted as a function of the average increase in potential over its resting value during that time. (The top line of Fig. 7 is one of the records from which the data were obtained.)

of the light intensity during the third second of a prolonged flash. As you can see the relation is approximately linear over a range of about $4.75 \log$ units, a change in illumination of about 50,000 times. $^{7}$

By counting the number of impulses during the third second of discharge the steady state frequency was obtained. Fig. 9 shows the approximately linear relationship between the slow potential and the frequency of discharge measured from the records used to prepare Fig. 8.

Thus we have a logarithmic relationship between light intensity and the slow depolarization of the receptor cell and a linear relationship between depolarization and spike frequency. From the well known properties of electric currents in stimulating nervous tissue it is tempting to postulate that the action of light amplified by some electrochemical process that we do not yet understand causes a graded electrical depolarization of the receptor cell. The currents thus produced would stimulate the pacemaker mechanism. That the slow potential is the precursor of the spikes is supported by several lines of evidence.

${ }^{7}$ A similar linear relationship between depolarization and spike frequency has been found in frog muscle spindles (Katz, 1950). 


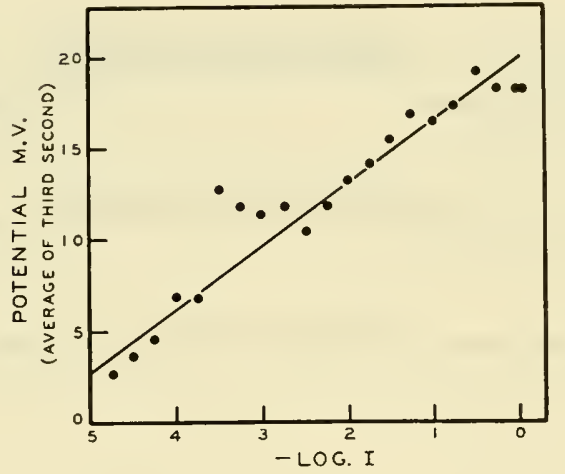

FIG. 9. Elevation in potential above the resting level in a Limulus photoreceptor cell as a function of the logarithm of illumination. (From the same records as Fig. 8.)

First, a large electrode would sometimes injure a receptor cell so that a very rapid spike discharge was produced after which spike activity could no longer be elicited. The slow potential still appeared in response to light. ${ }^{8}$

Secondly, slow potentials have been found associated with the spike discharge in many other receptors and have been interpreted as generator potentials which remain after abolition of spike activity by means of local anesthetics. Examples are the experiments on the muscle spindles of frogs (Katz, 1950) and on the stretch receptor in the abdominal segments of the crayfish (Eyzaguirre and Kuffler, 1955). In both of these cases it was possible to abolish spike activity with local anesthetics and still obtain the slow potentials.

Thirdly, we have made some experiments in which polarizing current was passed through the recording microelectrode (MacNichol et al., 1953). A ten thousand megohm resistor was attached at one end to the micropipette electrode and the other end to a source of potential that could be varied with respect to the reference electrode which was connected to the return lead of the amplifier. Thus we could record and stimulate through the same pipette simultaneously.

When the electrode was placed intracellularly a positive potential applied to the resistor caused a current to flow in such a direction as to increase the polarization of the cell membrane. Potentials applied in the other direction caused current to flow in such a direction as to depolarize the membrane. Fig. 10 shows oscillograms obtained with such a preparation.

Initially, the cell was discharging spontaneously due to injury by the electrode. Positive potentials speeded the discharge and negative potentials slowed it down. Fig. 11 shows the nearly linear relationship between the change in frequency and the applied current.

${ }^{8}$ This phenomenon was observed much earlier by Hartline using external electrodes (Hartline et al, 1952). 


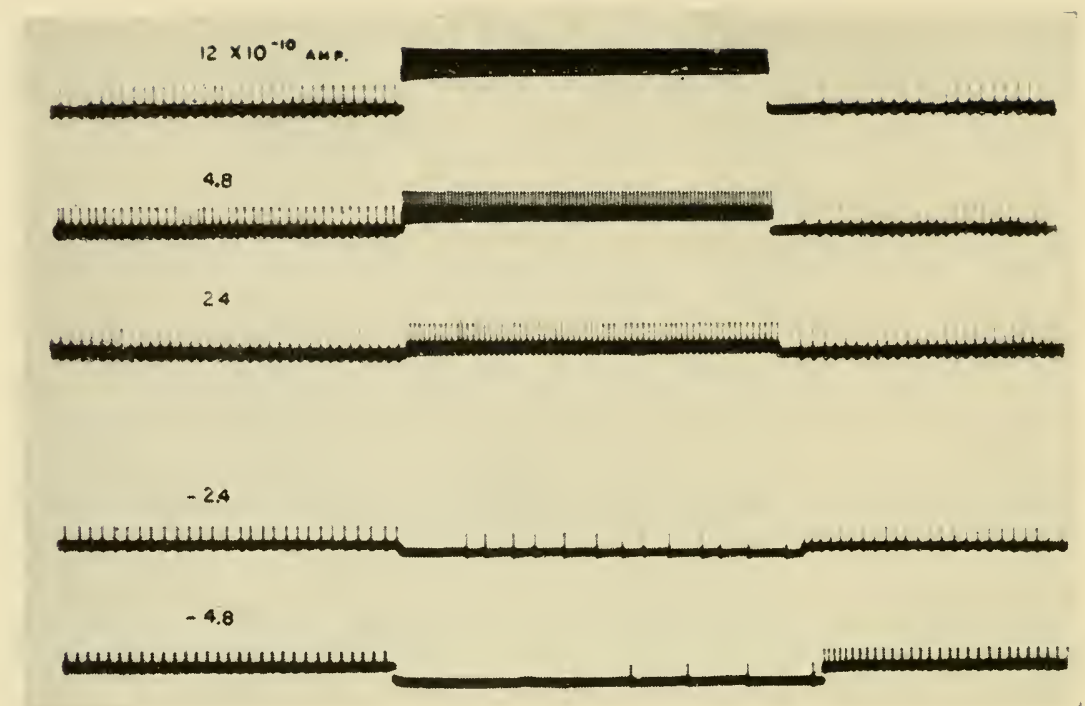

Fig. 10. Effects of electrical polarization of a Limulus photoreceptor through the same micropipette electrode used to record the discharge. The cell was firing spontaneously when the current was applied. Upward deflection indicates that the pipette became more positive. D.C. amplification; impulses approximately $40 \mathrm{MV}$. in height; electrode resistance approximately 20 megohms. Resistor through which the current was applied $10^{10}$ ohms.

Only about $10^{-10}$ ampere was required to produce a very noticeable effect. Here I should like to insert a word of warning to electrophysiologists since many physiological amplifiers have grid currents of this magnitude. This experiment indicates that the grid current may alter the properties of some excitable cells. Besides illumination and electric current several other factors have been shown to influence the spike discharge frequency in the Limulus photoreceptors. These are: The state of light or dark adaptation, changes in calcium and potassium ion concentration, and activity of adjacent units.

Fig. 12 shows the effects of addition of calcium and potassium ions to the fluid in which the receptors were bathed (MacNichol, 1952). The recording here was taken back in the nerve so that we were not able to follow the slow potential; just the spike activity. We have not yet repeated these experiments using micropipettes. The eye was placed in a flowing control solution and allowed to dark adapt. It was then subjected to hundredth second flashes of light at regular intervals. The intensity of the flashes was increased in small steps and the number of responses to each test flash recorded. After the most intense flash a new bathing solution was applied and the eye allowed to equilibrate in the dark for about a half hour after which an identical test run was made. This 


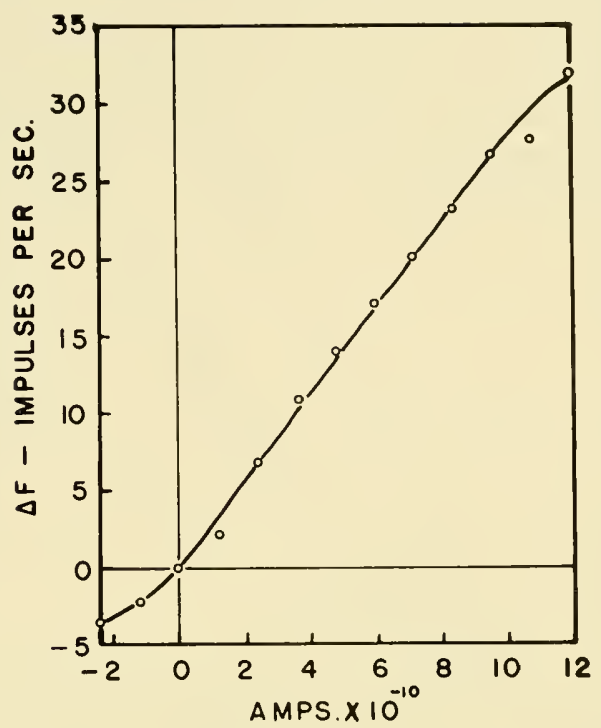

Fig. 11. Relation between electrical polarization and change in frequency of discharge of a spontaneously firing photoreceptor. The records of Fig. 10 are some of those used in preparing this figure.

process was repeated with various test and control solutions until failure of conduction in the nerve fiber terminated the experiment.

The figure was obtained from a sequence of four test runs. The ordinate represents the number of impulses discharged in response to a flash and the abcissa the logarithm of the light intensity. The first run represented by the filled circles was a control. In the second, represented by the open circles, more than the physiological concentration of potassium was used in the bathing Huid. In the third, represented by the solid triangles, excess calcium was used, and the fourth run, indicated by the open triangles, was taken after the eye was returned to the control solution.

The effect of potassium was to decrease the threshold so that impulses were discharged in response to weaker light, and to increase the number of impulses added by a given change in light intensity. The effects of calcium were just the reverse which is not surprising in view of the well known antagonism between potassium and calcium in other tissues. Potassium-free solutions increased the threshold and decreased the slope of the curve; whereas, in calcium-free solutions the receptor behaved very much as it did in increased potassium. Both increase of potassium and withdrawal of calcium caused spontaneous activity in the lark.

An unexpected result of these experiments was a specific effect of potassium 


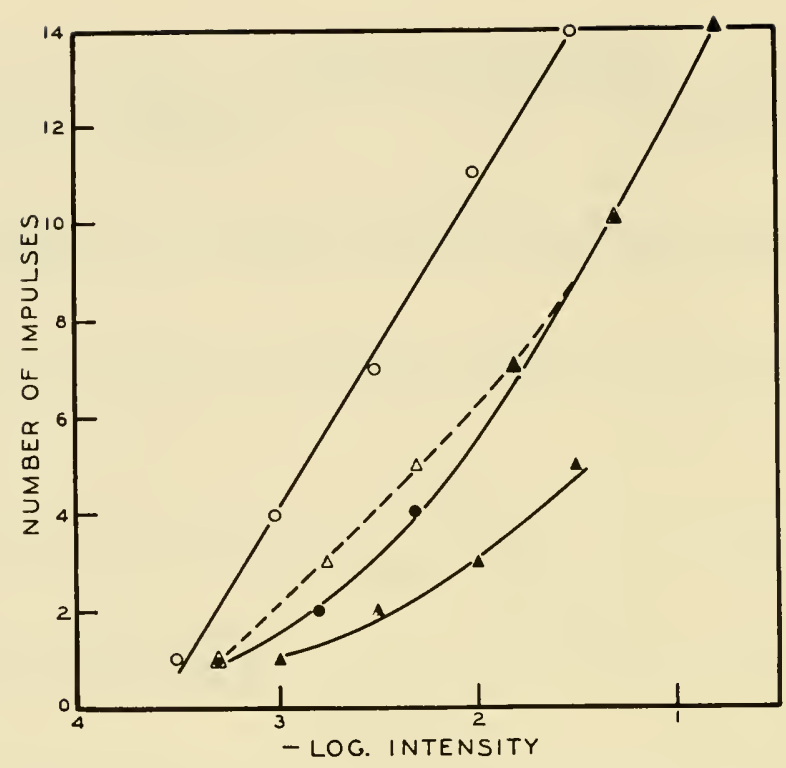

FIG. 12. Family of curves representing the effects of excess potassium and calcium in the external medium on the number of impulses discharged by a single unit in the Limulus optic nerve in response to flashes of light of variable intensity and 0.02 sec. duration. (๑) Normal salt concentration. (O) $10 \mathrm{mM} \mathrm{KCl}$ added; other salts normal.

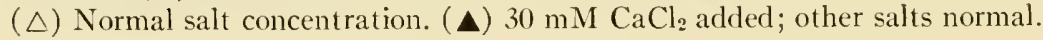

on the rate of dark adaptation. Fig. 13 shows the rate of recovery after ten minutes exposure to a very bright light. Brief test flashes were repeated regularly at progressively decreasing intensities and the time of appearance of the first impulses at each light level noted.

The logarithm of the threshold intensity was plotted against time in the dark. The first run shown by the open circles was a control, then excess potassium was used as shown by the right halfmoons. Then potassium-free solution was used (open triangles). Then excess potassium was given again (left halfmoons). Then calcium was withdrawn from the solution (open squares). The potassium was withdrawn again (closed triangles) and finally, a control was made (closed circles).

Clearly excess potassium speeded dark adaptation and its withdrawal slowed it down.

Indeed, in some experiments when potassium was withdrawn there was a prolonged discharge after the adapting light was turned off. The afterdischarge finally stopped and subsequently very little dark adaptation took place. It may be that during light adaptation potassium leaks out of the receptors which cannot dark adapt until it is restored from the external medium. That the effect is not due just to a change in threshold of the receptors is shown by the 


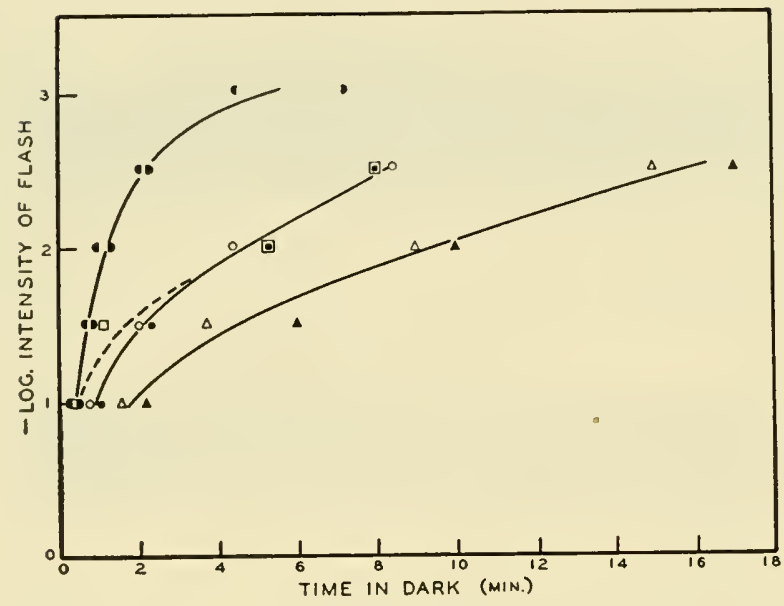

FIG. 13. Effects of changes in external potassium and calcium concentration upon the rate of dark adaptation of a Limulus photoreceptor unit. After equilibration in each test solution the receptor was subjected to a bright light ( 0 log units on the curve) for 10 minutes after which test flashes of $0.02 \mathrm{sec}$. duration were presented at progressively decreasing intensities and the time of occurrence of the first impulse at each intensity noted. (O) (O) Normal salt concentration. (D) (1) $20 \mathrm{mM}$ potassium added, other salts normal. $(\triangle)(\boldsymbol{\Delta})$ No potassium in solution, other salts normal. ( $\square$ ) No calcium in solution, other salts normal.

open squares and the dotted curve relating to the run in which calcium was decreased. Although before light adaptation the preparation was even more sensitive than when potassium was added, in fact it was firing spontaneously, it dark adapted less rapidly. Except in the initial stages the threshold followed along the control curves rather than along the curve representing the effects of increased potassium. Therefore it appears that potassium exerts a specific effect on the rate of dark adaptation rather than just the general increase in sensitivity that you would expect to get with either increased potassium or decreased calcium. It has been suggested that potassium exerts its effect on cells by partially depolarizing the membrane, while calcium affects its permeability. How such changes might account for differences in the rate of adaptation we do not know. We at first thought that potassium might have a specific effect upon the bleaching and regeneration of the visual pigment. However, from other work, it now seems that the bulk of the evidence indicates that light and dark adaptation are exerted primarily by events subsequent to the bleaching of the photopigment and not by the bleaching itself as was formerly supposed.

Hartline, Milne and Wagman (1947) have done some experiments that indicate that the number of quanta required to stimulate Limulus receptors increases with light adaptation. If adaptation was produced by bleaching, a con- 
stant number of quanta would be required. More direct evidence has been provided by some experiments of Rushton $(1952,1954)$ and of Weale (1953). They measured the bleaching of the photopigments in intact mammalian eyes spectrophotometrically and at the same time measured the changes in the visual threshold. These experiments showed that enormous changes in thresholds were produced when only a small fraction of the pigments was bleached.

Finally, Wald (1954) has made artificial eyes which duplicated as nearly as possible the conditions in the human eye. They had a layer of photopigment at the back which was estimated to have the same concentration as in the human eye. He exposed these eyes to light and measured the rate of bleaching spectrophotometrically. He then placed the eyes of human observers in front of his light source and measured their rate of adaptation. He found that light sufficient to change the observers' thresholds more than a thousand fold bleached less than one percent of the photopigment in the artificial eyes.

Wald has postulated a structural arrangement in the outer segments of the receptors that could produce large changes in threshold by bleaching only a small fraction of the molecules of photopigment. There is little evidence for this theory at the present time but it presents an attractive possibility.

At the present time we do not know where the change in concentration of the potassium ion exerts its effect. We must investigate it further. It appears that very little of light and dark adaptation can take place in the trigger mechanism which generates the impulses since some rather crude preliminary experiments indicate that the slow potentials recorded from the microelectrodes appear to follow a course of light and dark adaptation parallel to changes in the frequency of the spike discharge. It must be concluded that dark adaptation takes place in the electrochemical amplifying mechanism activated by the splitting of the photopigment and resulting in the production of the graded slow potential.

This mechanism must be complex since it requires some time to act, as shown by Hartline's (1934) experiments on the critical duration. He found that the response to a short flash of light takes place wholly in the dark as shown in Fig. 14. If a short flash of light is given there is a latent period before any impulses are generated or any slow potential appears. In the dark adapted animal this time can be as long as a second and usually is at least some tens of milliseconds. Furthermore the duration of the latent period is determined only by events taking place near its beginning as shown in the following experiment. Two flashes of light separated by a variable time are presented to the eye. If the second flash appears within an interval known as the critical duration it will shorten the latent period of the first response. If it appears after the critical interval it will have no effect on the first response but can affect the time of occurrence of subsequent impulses. The critical duration is from $\frac{1}{3}$ to $1 / 2$ the duration of the latent period. Thus there is this considerable period of time in 

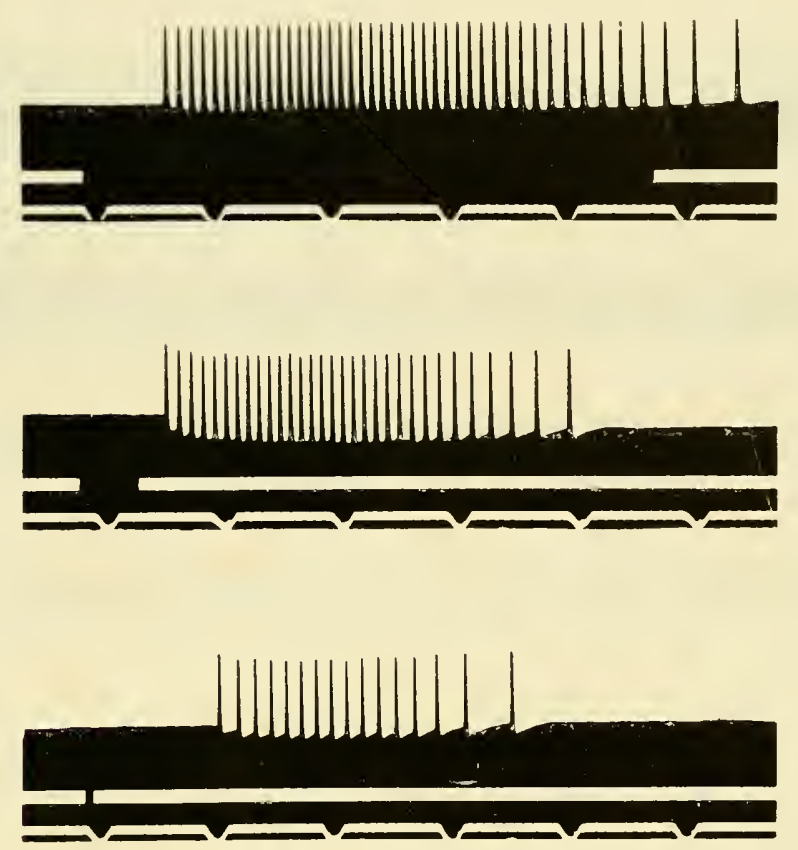

FIG. 14. Discharge of impulses in a single optic nerve fiber of Limulus, in response to exposures of the eye to light of the same intensity for clurations of $0.97 \mathrm{sec}, 0.096$ sec and $0.010 \mathrm{sec}$. (top to bottom). Signal marking period of exposure blackens out white line above time marks. Time in $15 \mathrm{sec}$. The times from the onset of illumination to the successive occurrences of corresponding impulses in the upper and middle records were the same for the first 10 impulses. From the 11 th impulse on, the occurrences were significantly earlier in the upper record than in the middle. Therefore, $0.096 \mathrm{sec}$ was the "critical duration" for the 10th impulse, for which the time of occurrence $=0.32 \mathrm{sec}$. For the 1 st impulse (time of occurrence $=0.1+\mathrm{sec}$ ) the critical duration at this intensity was $0.04 \mathrm{sec}$. (Reproduced from Hartline et al. 1952.)

which nothing observable happens but during which the response is already determined and we suppose that this must be occupied by intermediate processes, the nature of which is yet unknown.

Finally, I should like to mention the inhibitory effect investigated by Hartline (1949) and by Hartline and Ratlif (1955). Illumination of adjacent elements causes a decrease in frequency of a unit responding to illumination. This is shown in Fig. 15. The ommatidium from which the recording was made was illuminated by a small spot of light which was turned on several seconds earlier than the portion of the record shown in the slide. The black band indicates the time of illumination of a neighboring area of the eye by a separate spot of light. You can see that there is a decrease in the frequency of response during the illumination of the neighboring elements. When that illumination is 


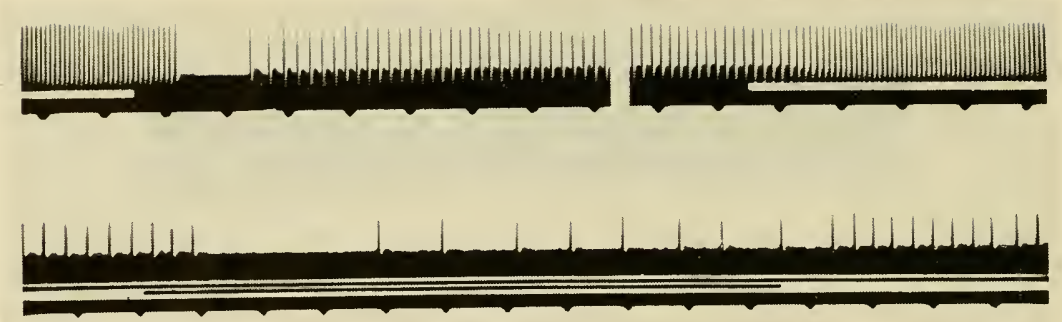

Fig. 15. Inhibition of impulses discharged in single units in the Limulus eye by illumination of neighboring units. The black band above the time trace indicates the duration of the inhibitory illumination. Tine marks indicate fifths of a second. (Figure courtesy of H. K. Hartline.)

turned off then the frequency speeds up to nearly its initial value. The two records were made at different intensities of illumination. That the inhibitory effect is produced by activity of the neural elements rather than by the action of some photochemically liberated substance was shown by Tomita (to be published) who found that antidromic stimulation of the nerve fibers arising from adjacent units would also produce an inhibitory effect. In other words, no light was necessary to produce inhibition; only the activity of adjacent units was required as shown in Fig. 16.

Histology shows that in the Limulus eye there are no ganglion cells and that there are axonal cross connections. These were shown in Fig. 6.

The receptor cell is somehow affected by these comnections between the axons, since we have found that the inhibitory effect can be picked up just as well as a frequency change by a micropipette inserted into an ommatidium as it can back in the optic nerve.

It is probable that the inhibitory effect is exerted directly upon the impulse generating mechanism since inhibition is produced upon illumination of nearby elements irrespective of the method of stimulating the active element. We have found that trains of impulses produced by injury, changes in chemical environment, electrical stimulation, or by after-discharge subsequent to intense illumination can be inhibited as easily as those produced during direct illumination.

A tentative hypothesis is that the inhibitory connections exert their effects by altering the state of polarization of the region at which impulses originate. Unfortunately we do not yet know where this region is. There is some evidence that it is in the axon near its junction with the cell body. In the first place the spike potentials recorded from micropipettes are never as great as the resting potential of the receptor cell (resting potential $50 \mathrm{mv}$; spikes $25-40 \mathrm{mr}$.) even when there is no injury discharge and the cell appears to be functioning normally in every way. This would indicate that the impulses never invade the 


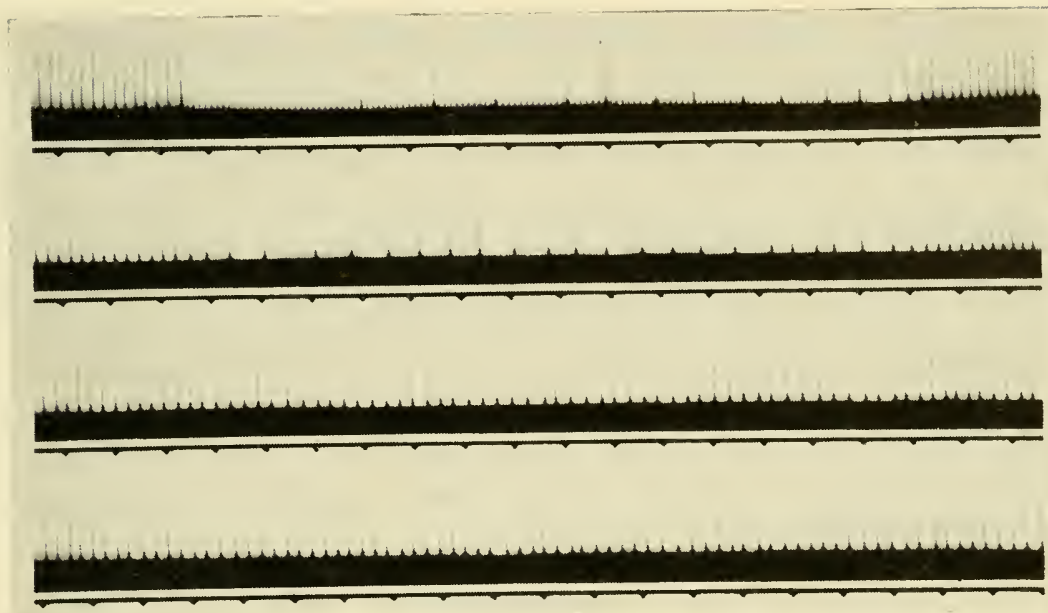

FIG. 16. Inhibition of discharge of a Limulus photoreceptor unit by antidromic stimulation of the nerve fibers leading from neighboring units. The electrical stimuli were applied at constant frequency. The records are arranged in order of increasing strength of the antidromic stimulus from bottom to top. The stimulus artifacts can be seen as small spikes in the top record. (Figure courtesy of T. Tomita.)

cell body in this preparation. Secondly, when the pipette is just outside the receptor cell the small spikes that are recorded are positive as are the large spikes recorded from within the cell. If the spikes actively depolarized the cell we should expect the spikes recorded from just outside to be negative as they are when electrodes are just outside spinal motor neurons. Thirdly, Hartline (1952) has recorded potentials with an electrode placed in various positions on the surface of an isolated but unopened ommatidium. At a critical location on the neck of the ommatidium the spikes reversed in sign becoming positive when the electrode was moved toward the cornea and negative as it was moved toward the nerve. Unfortunately we have not been able to repeat this experiment with an opened ommatidium in order to see where this reversal occurred with reference to the internal structures. We have thus far been able to obtain only positive spikes from electrodes inserted into ommatidia.

We have also looked for slow potentials that might be associated with the inhibitory effect. There is some indication that they exist but are usually masked by larger potentials generated by stray illumination reaching the active ommatidium. They would be expected to be small if they resulted from an electrotonic spread into the cell body from the region of the axon under the cross connections.

This is as far as we have gotten at present. We badly need some way of determining the location of the tip of the microelectrode within an ommatidium with respect to the histologically identifiable structures. It may be that micro- 
dissection of the husk of the ommatidium is possible or that a digestion with enzymes will facilitate its removal. This still remains to be tried.

We may also get clues from the work on other receptors having analogous properties. The beautiful work of Kuffer (1954) and Eyzaguirre and Kuffler (1955) on the abdominal stretch receptors of crustaceans in many ways parallels our own but is far more complete. Here they have shown that a single cell is capable of giving a slow potential in response to a mechanical stimulus, that the slow potential appears to cause the generation of spikes, and that there are direct inhibitory connections to the cell. Since the tissue is transparent and the cell is large, they are able to place their microelectrodes in axon, soma and dendrites at will. We hope that the type of activity that they are obtaining in different portions of the cell will give us clues as to where our electrodes are placed even though we cannot see their tips.

\section{References}

Adrian, E. D. 1935. The Mechanism of Nervous Action. Philadelphia. The University of Pennsylvania Press. p. 103.

Eyzaguirre, C. and S. W. Kuffler. 1955. Processes of excitation in the dendrites and in the soma of single isolated sensory nerve cells of the lobster and crayfish. J. Gen. Physiol. 39: 87-18t.

Graham, J. and R. W. Gerard. 1946. Membrane potential and excitation of impaled single muscle fibers. J. Cell. Comp. Physiol. 28: 99-117.

Granit, R. 1955. Receptors and Sensory Perception. New Haven. Yale University Press. 369p.

Hartline, H. K. 1934. Intensity and duration in the excitation of single photoreceptor units. J. Cell. Comp. Physiol. 5: 229-247.

Hartline, H. K. 1949. Inhibition of activity of visual receptors by illuminating nearby retinal areas in the Limulus eye. Fed. Proc. 8: 69.

Hartline, H. K., L. J. Milne and I. H. Wagman. 1947. Fluctuation of the response of single visual sense cells. Fed. Proc. 6: 124 .

Hartline, H. K., H. G. Wagner and E. F. MacNichol, Jr. 1952. The peripheral origin of nervous activity in the visual system. Cold Spring Harbor Symp. Quant. Biol. 17: 125-141.

Hartline, H. K. and F. Ratlif. 1955. The organization of neural activity in the eye. Symposium on Physiological Psychology. ONR Symposium Report ACR-1: $92-101$.

Katz, B. 1950. Depolarization of sensory terminals and the initiation of impulses in the muscle spindle. J. Physiol. 111: 261-282.

Kuffer, S. W. 1954. Mechanisms of activation and motor control of stretch receptors of lobster and crayfish. J. Neurophysiol. 17: 558-57t.

MacNichol, E. F. Jr. 1952. The effects of cations upon photoreceptors. Ph.D. Dissertation. Unpublished. The Johns Hopkins University.

MacNichol, E. F. Jr., H. G. Wagner and H. K. Hartline. 1953. Electrical activity recorded within single ommatidia of the eve of Limulus. Abs. XIX International Physiological Congress. Montreal, Canada.

MacNichol, E. F. Jr. and H. G. Wagner. 1954. A high impedance input circuit suitable for electrophysiological recording from micropipette electrodes. Research Report. 
Project NM 000 019.03.01. Vol. 12: 97-118. National Naval Medical Research Institute. Bethesrla, Md.

Rushton, W. A. H. 1952. Apparatus for analyzing the light reflected from the eye of the cat. J. Physiol. 117: $47 \mathrm{P}$.

Rushton, W. A. H. and R. D. Cohen. 1954. Visual purple level and the course of dark adaptation. Nature 173: 301-302.

Wald, G. 1954. On the mechanism of the visual threshold and visuat adaptation. Science 119: 887-892.

Weate, R. A. 1953. Photochemical reactions in the living cat's retina. J. Physiol. 122: $322-323$.

\section{Discussion}

Led by II. D. McElroY

When I was asked to follow as discussant on this morning's program, I was asked to talk about the biochemical aspects of this phenomenon. Dr. Gerard said we should comment on points which have been presented by the two previous speakers. That makes it a little difficult for me to put into biochemical terms, what has already been covered this morning. I think one of the points Dr. Grenell wanted me to talk about, and I will do this in about three or four minutes, is the fact that you have a charged system here which under appropriate stimulation can give a rapid response and return to normal. The question is: Do we have any biochemical information which is analogous to this process? Now, as you know, I am interested in the problem of conversion of chemical energy into light energy and reverse. In working with the firefly, trying to explain the flash of the firefly, you practically get into the same problems which you are talking about this morning. You stimulate a nerve leading to the light organ in the firefly and you get a flash of light and this returns to the normal state in about two seconds.

Now, the question is, can we isolate and identify all the chemical components of this system and can we duplicate and explain the flash mechanism and its control in an isolated system? We think we can and I will just briefly give you the information we have at the present time, how we maintain the light at a low steady state level in the test tube and with appropriate stimulation get a flash which is super-imposable upon the flash of the firefly from the intact organism.

The light intensity, which is really the rate of the reaction in this case, is proportional to the concentration of a single protein component (luciferase), a substrate molecule which is called luciferin, ATP, magnesium and oxygen. If one puts all of these compounds at saturation with respect to the enzyme, one obtains in less than a second a light which stays at a reasonably steady state level. If one now adds additional protein to the system, and inorganic pyro- 
phosphatase is very potent, the light intensity is depressed proportionally to the amount of protein that is added. One can add enough protein to the system to bring the intensity down to the order of one or two per cent of the initial light intensity. This system will continue to luminesce for hours. If one adds inorganic pyrophosphate to the system, one obtains a flash of light the duration of which depends upon the rate of hydrolysis of pyrophosphate. If a very small amount of pyrophosphate is added with a high concentration of pyrophosphatase, one gets a spark of light and if this is recorded on a cathode ray oscillograph you get a typical flash which is super-imposable on the normal firefly's flash.

\section{Chiatruan Gerard: Of what duration?}

Dr. McElrov: On the order of two seconds, in this case. This is an example of how one can depress enzymatic activity, i.e., hold it at a low, steady state level with all the elements there that are necessary for light emission. If one puts in a stimulant, if you want to call adding pyrophosphate a stimulus, a response is observed which is identical to that of the firefly. There is only one other way you can obtain the flash in this system and that is by placing the system under anaerobic conditions and then letting in oxygen. Under these conditions one obtains a flash but it is about ten times too long in duration. We have been unable at present to obtain a very rapid flash by this procedure. We have one hypothesis which fits an awful lot of data collected from this system. It is now clear that the ATP reacts with luciferin to form an active luciferin and pyrophosphate. The active luciferin in the presence of oxygen is oxidized and is converted eventually into an excited molecule. This excited molecule then decomposes into oxyluciferin, adenylic acid and light. Oxyluciferin turns out to be a potent inhibitor of luciferase as far as light emission is concerned. However, oxyluciferin, itself, can react with ATP to form pyrophosphate and presumably adenyl-oxyluciferin, the latter being the competitor with active luciferin for the luciferase. Pyrophosphatase depresses the light intensity by favoring the formation of the inhibitor while the latter pyrophosphate stimulates luminescence by removal of the inhibitor.

I could go into more details and supporting evidence for this but I think it is enough to show how one can have in a biological system all of the reactants that are necessary for carrying out a process without the event actually occurring. It is a charged but depressed system requiring appropriate stimuli to bring it back temporarily to a very active state.

The addition of inhibitors like calcium which has a very interesting effect on this system, it prolongs the light simply because it inhibits the inorganic pyrophosphatase action. It might have some analogies with the in riro systems discussed this morning.

Dr. Robert Grenell (Psychiatric Institute, University of Maryland): 
Would you have any suggestions about the relationship of such a system to an in rivo system as far as the time factor is concerned?

1)R. McElroy: As I say, with the appropriate pyrophosphatase concentration and by our adding pyrophosphate we can get the same time relationship as observed in the intact firetly.

We have to trace the question back a little further now as to how we get inorganic pyrophosphate formed. One can pick any number of ways of getting it from known biochemical reactions. It is also well known that protein bound pyrophosphate occurs in several different organs and cells. 'The fact that you get a temperature independence in a lot of these processes may mean merely in this case a liberation of pyrophosphate by some other process. I might add that the rate of decline of luminescence is essentially temperature independent. Now what this has to do with nerve conduction and the type of trigger mechanism we are talking about this morning is anybody's guess. But I am convinced that this is the way the firefly controls its flash and since this is under nervous control, I guess that is the reason I can discuss it.

I should have made one point; namely, we haven't done the experiments, they would be interesting ones to do, to add smaller amounts of pyrophosphate. One could lower this two seconds if necessary. We were interested in duplicating the system that occurs in the firefly.

Dr. Leo Abood (University of Illinois): What Dr. McElroy said in connection with pyrophosphate and pyrophosphatase is extremely interesting in light of some findings we made on frog sciatic nerves. We isolated and characterized inorganic pyrophosphate as well as inorganic pyrophosphatase in frog sciatic nerves and during stimulation of these nerves for periods up to 15 minutes to half an hour, we could observe a considerable increase in the inorganic pyrophosphate of nerve and by the use of tracers we have been able to find a correlation between this increase - that is, the entire turnover of pyrophosphate and the turnover of ATP's, suggesting that there is definitely a relationship between them. Also DPN does seem to increase in turnover during this time and the possibility is that the pyrophosphate may come from DPN and ATP. Now what the significance of that is, we do not know. It certainly is a fact.

Dr. Morales: As Dr. McElroy knows, I am sure, essentially the same story insofar as pyrophosphate is concerned is repeatable in muscle in thiamine and tetra-acetate. I think that it is a very interesting contribution to the control system, but it leaves open the matter of the energy source. I wonder, in his case, what is the substance that is consumed here? Is it presumably the L.H-2?

Dr. McElroy: The LH-2 is consumed, this you can show, but one must use low concentrations of this substrate. If one makes the LH-2 limiting then you get an ordinary enzyme reaction in which the light eventually goes out due to 
luciferin consumption. You can also show the same thing with ATP now, an observation we couldn't make before. But it means you have to go to very low ATP levels to show this quantitatively. Actually, it takes very little of this material to give a bright light. Energetically, this is a very sensitive system because you do not use very much material to get the energy for light production.

Dr. Morales: You mean that both are used up?

Dr. McElroy: Yes.

Dr. Morales: Then how do you have any ideas as to which is which?

Dr. McElroy: You mean in terms of the energy source?

Dr. Morales: Yes.

Dr. McElroy: Well, we need 70 kilo calories. This is getting into the mechanism of luminescence and I am sure that that has nothing to contribute to receptors. But I would just make one comment.

Chatrman Gerard: This is a typical transducer problem.

Dr. McElroy: Yes. We need 70 kilo calories for the peak light. The reaction between ATP and LH-2 leads to the formation of an active molecule which can be oxidized. We know this molecule is oxidized. It is not oxidized in the absence of ATP. ATP in some way brings about an activation for this oxidation. It is very doubtful that a straight oxidation of this type of molecule could give enough energy. So we feel that an organic peroxide is formed with one such active molecule and this, in turn, leads to a peroxidation of a second molecule. From such a peroxidation one can get roughly 70 kilo calories. We know that luciferin is the emitting molecule because it has a fluorescent spectrum which is identical to the chemiluminescent spectrum and that this changes with $\mathrm{pH}$. So this particular molecule is the one that becomes excited and the energy in this case, you might say comes not from ATP but primarily from an oxidation. One might say ATP contributes activation energy and the main energy for subsequent excitation comes from the peroxidation of the luciferin molecule.

Dr. MacNichol: I am very much struck by what Dr. McElroy has to say. I mean, here is apparently just the inverse of the photochemical reaction. IVald has shown that the condensation of protein, in the case of the eye, is an energy yielding reaction, so much energy is required and light energy gives a sufficient amount to split these apart. Now here you have these two active compounds, which combine in an energy yielding reaction and produce light. Is there anything in your system which would show where we go from there?

Dr. McElroy: I look at that as exactly the same as this. One has to, I think, in energy reaction distinguish between that and activation energy. If you have two molecules bound together, A and B, and you want them to go over to A plus $B$ with the energies given out - if I understand the situation 
correctly-what your light energy is doing is merely supplying activation energy. So you can look at this in the same way as pyrophosphate splitting, freeing a complex. What you are doing is freeing a complex which is separated from its products by activation energy.

Dr. MACNichoL: No, in this case the products are, I think, in a higher energy state.

Dr. McElrox: It is shown from retinene, from vitamin A for example, the equilibrium is way over toward the vitamin $A$ and to get the vitamin $A$ directly, you have to remove the retinene by a combination with protein which, again, is an energy yielding reaction.

DR. MACNICHOL: I am wondering whether any of that system has anything to do with the propagation of the impulse, getting back down to nerve impulses, whether it isn't really that in splitting off the retinene from the protein when light is absorbed that reactive sites on the protein are uncovered. These could then combine with something that belonged to the neural part of the system and cause stimulation. In fact, Wald has shown that in the bleaching of rhodopsin, sulfhydryl groups are liberated. These are of course highly reactive. The question is what do they react with and what happens then?

Dr. Lawrexce Stark (School of Medicine, Yale University): I have a comment to make about the analogy between the trapped muscle physiology and trapped nerve physiology and that is, associating some chemical reaction with the process just because of the sequential change. We are all aware of the pitfalls of the lactic acid story about 25 years ago, and now the ATP ideas which have been gathered into plausible hypotheses seem to be failing in some recent work which has shown that ATP does not seem to change during the actual muscle twitch; it may not be responsible for the supplying of energy.

Dr. McElroy: I would like to challenge that statement, that latter one particularly. For 25 years, I have thought that ATP reacts with muscle protein to form a phosphorylated muscle protein and that if you could isolate this and get away from ATP one could show a contraction for a short period of duration of the liberation of phosphate without a break of ATP. I really do not see anything from the experiments of Momaerts and Hill, wasn't it? and Krebs, that negates the idea that ATP is the essential reacting agent in muscle contraction.

DR. STARK: But it may not be used up in supplying the energy.

Dr. McElroy: I don't think a lot of people have seriously said that. I think a lot of people have read that into early statements, that ATP was broken down at the time of contraction. A lot of people never had that notion who are working in muscle physiology.

Dr. Stark: A lot of people did.

Dr. McElroy: I think this is just a misstatement. I think this is a misinterpretation of the statements, really. 
Dr. StARk: We are in agreement then?

Dr. McElroy: Well, I still think that it fits in with ATP as being an immediate energy source.

Dr. Mulliss: I would like to ask Drs. McElroy and MacNichol about two of the points that arose in the discussion. Dr. McElroy says that you have to release this pyrophosphate in order to stimulate the reaction and presumably you have to have this thing taken up in order to get the thing back to normal again. That just pushes the problem of where the excitation is off to what releases pyrophosphate into the system, and so we should start, then, at that level, rather than at this advanced one.

Dr. McElroy: Dr. Mullins has brought up the point that perhaps retinene, when it is liberated, is the thing which is responsible for activation. I think that Dr. Wald feels that it is more likely the protein, such as the sulfhydry groups that are uncovered and which are then reactive and combined with something, perhaps, in the membrane which triggers off the photoelectrochemical mechanism. The only reason for postulating this is because of the delay in response. If one is at threshold conditions and ultimate sensitivity the reaction is linited by the diffusion time from wherever it happens-wherever you happen to break off such a single molecule-it may be deep in the receptor or it may be near the surface and so you should find some variability in that time; it shouldn't always be just one second.

DR. Fuortes: In your data you found that there were small spikes, or large spikes and small slow potentials. I thought the slow potential was perhaps large enough even when you had the small spikes. Is there a way to explain this? Are the two things generating on a different basis? Why are the spikes small where the slow potential is large? Is that, by any chance, damage due to a particular structure? Concerning the effect of direct current stimulation, are direct currents active in changing the figures of the charge wherever the direct current is located? Or, are they active when it is repeated in structures that produce the slow potential? My third question concerns the cross connections that give inhibition in the one receptor. Is anything known about where these terminations go on the receptacles except for the physiologicals?

Chairman Gerard: May I ask another question? Have you any idea what the retinula cells are doing there?

Dr. MAcNichol: I wish I knew. The axons, as far as we see, do not conduct anything and they may be purely some kind of an evolutionary accident. They may have been functional in some ancestor of the Limulus.

Chatructin Gerard: I am sorry, I didn't mean to divert you.

Dr. MACNicnol: Let me see if I can remember these three questions, now. When we get in some regions of the cell we get these enormous slow potentials; they are tens of millivolts and where we have the large spike activity we do not see more than a millivolt or two of what might be a generator potential below 
these 30 or 40 millivolt spikes. I think this is probably just a difference in the location of the two things.

The distal process of the eccentric cell, whatever it is, is quite long and thin. If the slow potential occurs here and if this is where our electrode is we get a large slow potential. But if we have an electrode in this large cell body, due to current flow through the membrane, the attenuation of this slow potential is likely to be very great.

DR. Mulliss: There are two interesting implications. One is the spikes are not conducted backward. We can see spikes up there.

Dr. MAcNichol: Apparently they do not invade the distal process.

Dr. Mulliss: Secondly, the electronic plane is very sharp.

DR. MACNichoL: I have been studying this recently, putting electrodes on the nerve back a ways, back several millimeters and we find that we can get slow potentials with the spikes superimposed upon them. And the records obtained back here look exactly like those we get with the micropipette except that the slow potentials are small. The spikes will go way off scale if we have sufficient amplifier gain to see the slow potentials. But the slow potentials seem to have the same quantitative relationship to the light intensity and to the frequency of discharge that they do further back in the system. We have also been able to pick up sub-liminal slow potentials. That is, if we put a short tlash of light in, we will get this rise in slow potential below threshold for the firing off of spikes. The slow potential starts up and then decays, or if it is a long flash the slow potential stays up. If you increase the light intensity, the slow potentials will get bigger and bigger and bigger and pretty soon we get spike. That looks as if there is evidence of a sub-liminal process.

In reply to, I think it was your first question about the electrical polarization, we did do a couple of experiments last summer in which we tried to relate the change in spike frequency to light and to current. That is, we had a calibrated amplifier and measured the resistance of our electrode, so that we could calculate for a given deflection in the base line produced by applying electrical polarization to the electrode how much the applied potential inside the cell had changed. We then applied different amounts of direct current and measured the spike frequency corresponding to various potentials between the inside and outside of the cell. We measured the change in potential across the cell produced by varying light intensity. What we found was, that with the particular location of our electrode that we had in this experiment, that about five times as much potential change was required to produce a given change in spike frequency in response to applied electric current as was required to get the same spike frequency by stimulating with light.

I think all this says is that we were just not stimulating at the most favorable place.

Dr. Miller has done a good bit of histology on the cross connections. He is 
going to work with Dr. Hartline during the next year and I hope he will get some more answers. It looks as if the cross fibers fan out into kind of a brush over the region where they surround the bundle of axons. There do not seem to be any connections between the ommatidia directly. They appear to occur entirely along the axons. It may be that these slow potentials, this electronic spread that $I$ have been measuring in the nerve is conducted through the cross connections over to the axons of other eccentric cells producing the inhibitory effect, but we do not have enough evidence yet to state this positively.

Dr. STARK: It seems to me that your explanation of the relationship between the spikes and slow potential is very similar to the local response of a nerve but different from what Dr. Fuortes was suggesting with the electrotonic slow change in the current in a different region from the spikes. Now, one point which might settle this is: Do your thresholds for light stimulation go up when you get the slow potential changes?

Dr. MAcNichoL: We always get the slow potential changes when we stimulate.

DR. STARK: You always do?

Dr. MacNichol: Yes.

DR. STARK: In other words, you would assume that your are injuring your membrane and therefore you would be getting a smaller safety factor and a larger slow potential before you got spike potentials; is that your reason?

Dr. MACNichol: No, I don't think so. Where we have gotten the large spikes, the preparation is quite stable. Sometimes we get spontaneous activity, but this is usually due to injury and often subsides after awhile.

Dr. BLUM: It seems we have a system in which you have a sort of a total maximum discharge, that is, some of the potential, the slow potential, in the spike is a sort of constant potential-you have a small D.C. potential and you get a large spike. You also, I believe, showed on the slide that the frequency of discharge was proportional to the slow potential and likewise the slow potential was proportional to the intensity. So you have two constants, $K_{1}$ and $K_{2}$. If you study these two constants at, say, different KCl's, or you produce calcium and you get spontaneous activity, you will get different constants. It seems to me that the relation between these constants may be used to obtain some clue as to the actual mechanism of the transduction that was going on. Because, assuming that the total change in potential, total change of slow potential, is a constant, then measuring the changes in $\mathrm{K}_{1}$ and $\mathrm{K}_{2}$, as a function of potassium or calcium, would give you some detailed clues as to what was happening in the inter-relation between the two.

Dr. MACNICHOL: I abandoned the work on the use of ions temporarily, I hope, when it looked as if the microelectrode technique was going to be useful and I have not gotten back to using that technique with the changes in ionic 
concentration. I want very much to do this and to try to study these very relationships that you have posed.

Dr. Jonx Hughes (National Institutes of Health): Regarding the relationship between the slow potential as recorded from ommatidia and the spike discharge from the fiber, I was wondering if you have been able to demonstrate an I.x'T. constant as was demonstrated by Dr. Hartline's experiments some years ago?

DR. MAcNichol: We haven't studied this specifically, but I am quite sure that it exists.

DR. DAN BRADLEY (National Institute of Mental Health): If I understood your talk, potassium ions seemed to be implicated in dark adaptation; is that right?

Dr. MacNichol: Yes.

DR. BRADLEY: Well, if this is so, we would expect that, if the light intensity in the light interval preceding the dark adaptation period were reduced sufficiently, the diffusion reaction would proceed faster than the light reaction, which would obviate the necessity for dark adaptation. Has such a threshold been observed?

DR. MACNichol: I haven't done any experiments, but I do not think that would bear on that. In our experiments I showed that we light adapted with as much light as we had available for ten minutes. But I have not done any work over longer periods at lower intensity.

Dr. GILBERT Ling (University of Illinois): I would like to make some comments. My ideas spring from our work on muscles. I wonder if Dr. Dethier would discuss with us whether such icleas are applicable. In this particular experimental material the idea was put forth several years ago that the selective ionic accumulation in the cells, as well as ionic permeability of the cell surface, could be explained by the hypotheses of a fixed charge system and the difference in interaction energy between such fixed charges and the definitely hydrated ions. The thesis was rather lengthy, but, in general, you may say that the sequence of ionic effectiveness or the sequence of ionic permeability and accumulation agree exactly with the sequence that you have seen as far as the cation threshold is concerned. Therefore, it turns, firstly, to why are these different ions-why do they have different effects in the strength of stimulation? The second is, why do they cause the excitation?

It is possible to think that because of the difference in the energy between the fixed charges, which themsetves are fixed, and the differently hydrated ions, which vary in polar energy according to their size, therefore a larger hydrated ion like sodium would have less effect than that of a smaller one, namely, potassium.

Now, the next question is, how would they initiate the electrical response? 
Enough has been said today about the activation of the potential, the generated potential. The slow potential in a certain part of the cells could at times initiate a chain of response. But we also think that at the end of the receptor, the cells are also sensitive to the action of, or combine differently with, anions as well as cations. The idea that we had formerly was that the reagents which produced such a change at a local point did so by inducing a permeability effect. Now, however, if you look back into the idea, the change of permeability would not immediately cause a change in the potential because it does not change the range of ionic distribution.

Recently we have shown that the potential generated at the cell surface seems to depend only upon microscopic depth. That is, that only the potassium ion at the cell surface will be responsible for the potential in the muscle. Consequently, would it be possible to think that your ions also affect this microscopic area, thereby producing a local depolarization, the generating potential, which in turn would bring about the activation?

Dr. Dethier: This could initiate a long discussion. In view of the time consideration I will not go on lengthily on this sulbject. Most of what I say would be sheer speculation.

Just let me say at this time, and I am willing to discuss it later at greater length, that acetylcholine does nothing at all in our system. And also, we can demonstrate no potassium, calcium, no ionic antagonism of any sort. Nobody working with taste has been able to. With respect to your last question, all I can say is that it is possible, but we have not shown it one way or the other. 


\title{
AGGREGATES OF NEURONS
}

\section{Affinity for Wool as an Indicator of Neuro- pharmacological Activity}

\author{
Roland Fischer ${ }^{1}$
}

Department of Public Ilealth of the Prozince of Saskatchewan, Universily IIospital, Saskatoon, Canada

I

$\mathrm{T}$

HE REASONS FOR CHOOSING wOol protein as a model of the structural surface of nervous receptors, possibly involved in some of the biochemical mechanisms of human behavior, are manifold:

(1) Hair or wool and nervous tissue are both of ectodermal origin. The former consists of $\alpha$-keratin, whereas neurokeratin is one of the most abundant of the proteins of nervous tissue. Both types of keratins have in common, among other things, their insolubility in acids as well as their resistance toward tryptic and peptic digestion (Fischer, 1953).

(2) It is believed (Block, 1951) that neurokeratin is possibly the protein in the neurofibrils, the filaments in the nerve cells and in the axon cylinder (Schmitt, et al, 1950).

(3) Certain basic (cationic) compounds display a strong affinity towards keratins of low sulphur content as shown by the gram-positiveness of epidermal and nervous tissue (Fischer, 1953; Bailey, 1950).

(4) More and more evidence is accumulating to support the theory that there is a similarity in behavior between wool and the protein component of certain cell membranes (Fischer, 1951; Fischer and Larose, 1952a; Fischer and Larose, 1952b; Larose and Fischer, 1952; Idem, 1953a; Idem, 1953b).

(5) Certain experiments confirmed the role of wool protein as a model simulating some of those receptors for which certain drugs appear to compete (Fischer, 1954). For instance, it was well known that methylene blue, which competes with acetylcholine in causing contraction of an isolated frog heart, can be seen easily by its blue color which is absorbed in to the tissue and which

\footnotetext{
${ }^{1}$ Research Biochemist, Psychiatric Research Unit; Lecturer, Department of Bio-
} chemistry, Medical School, University of Saskatchewan. 
even prolonged washing does not dislodge. This example of competitive absorption (displacement) could be simulated in a model experiment showing the high affinity of methylene blue chloride to wool, $\left(3.3 \times 10^{-2} \mathrm{mMoles} / 1 \mathrm{gm}\right.$. of wool $)$ whereas acetylcholine bromide could be washed out completely (desorbed) from the wool under the same experimental conditions.

\section{II}

Based on these premises, the affinity for wool $^{2}$ (Fischer, Hoelle and Seidenberg, 1951) of the following four basic compounds was determined at $\mathrm{pH}$ 5.2: mescaline hydrochloride, methedrine hydrochloride, lysergic acid monoethylamide (LAE) and lysergic acid diethylamide (LSD), the latter two in form of their methanoltartrates. These drugs, if administered to humans in the approximate range of dosages, $500 \mathrm{mg}$., $100 \mathrm{mg}$., $1 \mathrm{mg}$. and $100 \mathrm{mg}$. respectively, cause a model psychosis characterized by hallucinations and related phenomena of similar intensity and duration (Behringer, 1927; Mayer-(iross et al., 1951 and 1953; Stoll, 1947; Liddell and Weil-Malherbe, 1953; Solms, 1953; Fischer, 1946; Fischer, (ieorgi and Weber, 1951). The method of determining the affinity for wool used in this study gives only relative values but these were found to be proportional to the absolute values obtained by another method (Larose and Fischer, 1953b).

The results are presented in Fig. 1. The logarithms of dosage of each of the four compounds which causes a model psychosis of comparable intensity and duration are plotted against the amount of these compounds sorbed by wool. The results indicate that the higher the affinity of a drug for wool, the lower the amount of that drug required to cause hallucinations.

We a re inclined to attribute the increasing affinity of these drug for wool ${ }^{3}$ to their different degrees of specificity simulating a reversible inhibition of an equilibrium involved in the production of hallucinations.

We included only these four drugs in Fig. 1 because it is not easy to find other drugs which precipitate a reversible model psychosis with hallucinatory experience of comparable duration and intensity after administration of a single dose. Atabrine (mepacrine), for example, which is known to produce hallucinations and catatonic excitement ((ireiber, 1947; Szatmari) requires prolonged daily administration. ${ }^{4}$ Another antimalarial, of similar structure and action,

${ }^{2}$ The term wool is identical with that of intact wool used in some previous papers; (Fischer and Larose, 1952b).

${ }^{3}$ High affinity for wool does not mean the same for us as "selective absorption." An example: Bradley, 1951, has shown the selective absorption of one of the optical antipodes by wool from an aqueous or alcoholic solution containing both of the optical antipodes of resolvable acids.

${ }^{4}$ E.g. 3 gms. per day for 8 rlays (Halpern, et al, 1953). We found the affinity of wool for atabrine to be low, i.e. $0.9 \times 10^{-2} \mathrm{mMoles} / \mathrm{gm}$. This example suggest that compounds of low affinity for wool seem to display their biological activity only in high dosage. 


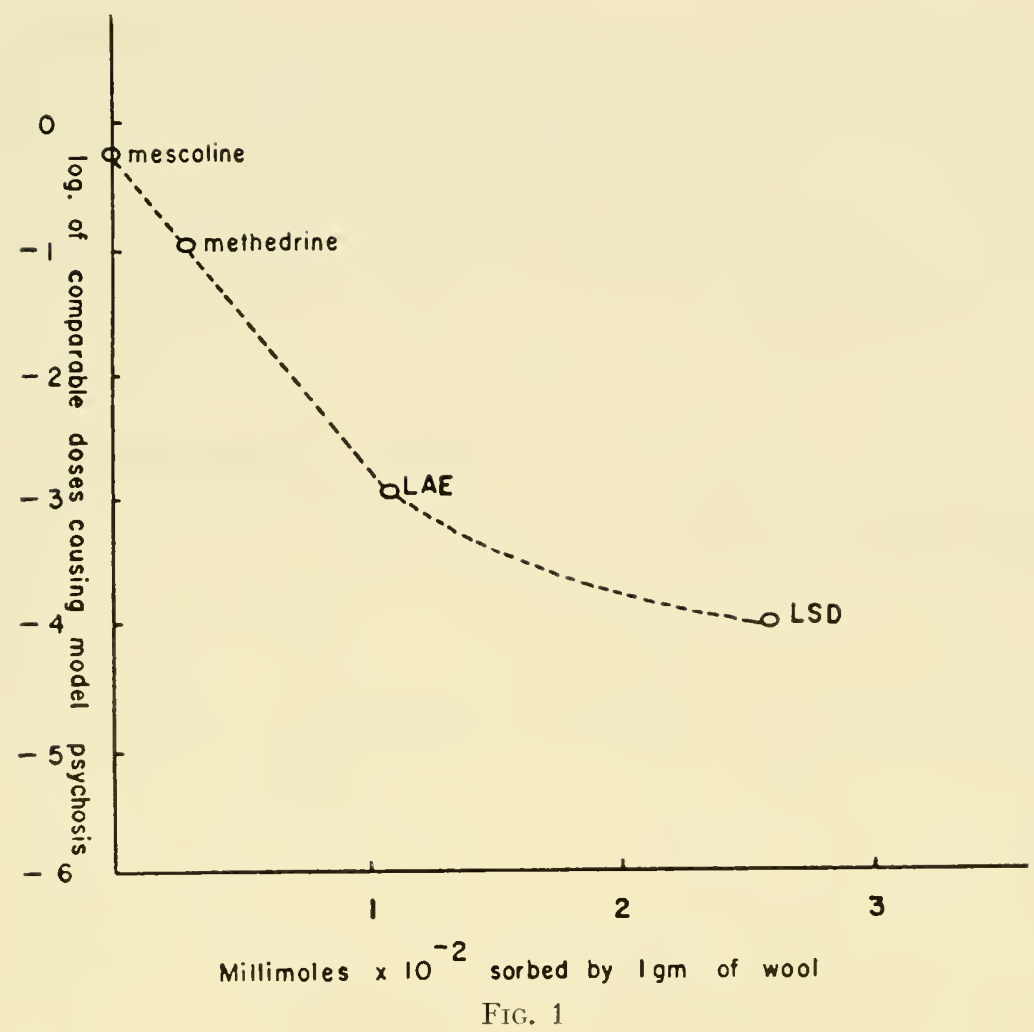

Affinity

i.e, average millimoles $\times 10^{-2}$ of drug sorbed by $1 \mathrm{gm}$. of wool

$\begin{array}{llllc}\text { Mescaline } & \ldots & \ldots & \ldots & 0-0.3 \\ \text { Metherlrine } & \ldots & \ldots & \ldots & 0.6 \\ \text { L.AE } & \ldots & \ldots & \ldots & 1.1 \\ \text { L.SD } & \ldots & \ldots & \ldots & 2.6\end{array}$

Single dose of drug

log. of single dose

$$
\begin{array}{rr}
0.5 & \mathrm{gm} . \\
0.1 & \mathrm{gm} . \\
1 & \mathrm{mg} . \\
100 & \mathrm{mg} .
\end{array}
$$

$-0.3$

$-1.0$

$-3.0$

Pentaquine, displays an affinity of $3.6 \times 10^{-2} \mathrm{mMoles}$ per $1 \mathrm{gm}$. of wool. If Pentaquine is administered daily at a dosage four times higher than usual $(0.12-$ $0.24 \mathrm{gm}$. day), it acts as an adrenergic blocking agent (Hoobler and Dontas, 1953). This feature seems to be, among others, a common characteristic of drugs capable of precipitating a model psychosis.

Liddell and Weil-Malherbe (1953) have shown that methedrine, as well as I.SI), blocks adrenergic activity by lowering the blood-epinephrine level, after a short initial increase, during the model psychosis. So might LAE, since it belongs to the family of ergot alkaloids, which are presumed to act centrally to inhibit sympathetic vasomotor tone (Hoobler and Dontas, 1953). However, it should be noted that the peripheral adrenolytic effects of LAE and LSD are about 300-2,000 times weaker than that of dihydroergotamine (Rothlin). 
Surgical sympathectomy (Harrer and Urban, 1953) or chemical (Dibenamine) (Walther-Büel, 1949) adrenergic blockage also produce psychotic experiences in certain subjects, possessing high epinephrine and nor-epinephrine (Nickerson et a]., 1953) levels ${ }^{5}$ to which they apparently are not adapted.

Hence, it appears that drugs which exhibit their central and or peripheral adrenergic blocking activity in smaller doses than e.g. Dibenamine, and simultaneously display a high affinity for (wool) protein, are able to cause hallucinations in very small doses.

\section{III}

\section{Speculations Related to Chemical Structure and Biological Activity}

In examining the chemical structure of some of the compounds displaying increasing affinity to wool, let us consider one aspect.

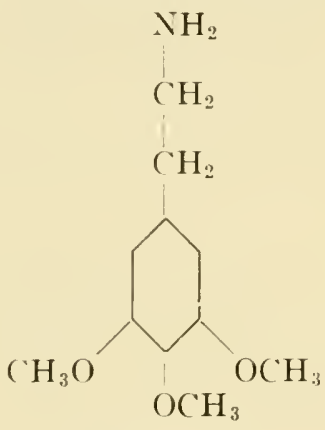

Mescaline Mol. w.: $211 \cdot 6$

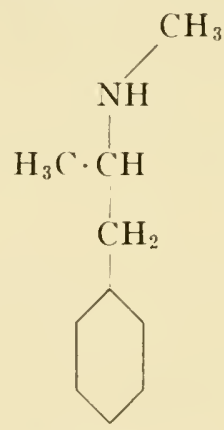

Methedrine Mol. w.: $149 \cdot 1$

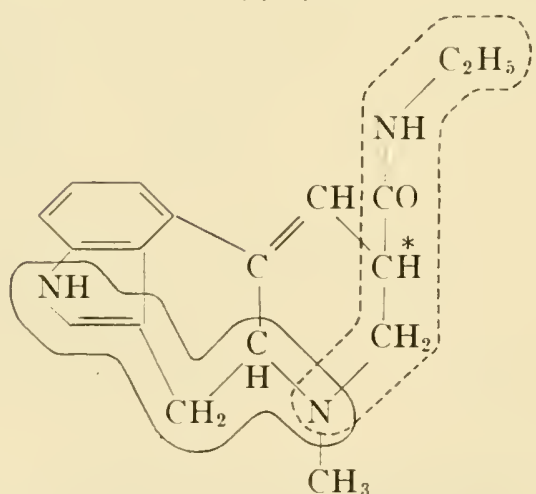

LAE

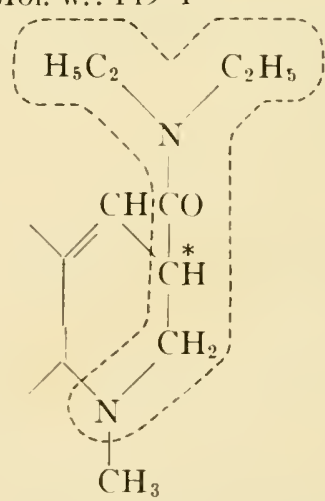

LSD

Mol. w.: 295

${ }^{5}$ Only about 20 per cent of hypertensive patients react to $0.5 \mathrm{gm}$. Dibenamine with a model psychosis (Walther-Büel, 1949). 
Bovet et al. (1953) have shown that the part mainly responsible for the specific pharmacodynamic activity of dehydrogenated ergot alkaloids is the molecular configuration centered at the primary $\mathrm{NH}_{2}$-group and its neighbourhood. We can extend this observation to almost all of our compounds involved in this report and compare in a deliberately oversimplified way the aforementioned groups on these compounds as one factor supposedly influencing their increasing absorption (affinity) for wool. From mescaline to methedrine: there is an additional monomethyl group on the primary amino $\mathrm{N}$. The third drug, LAE, also has an alkylated primary amino group (monoethyl) and then a three C-chain counting up to its additional $\mathrm{N}$ in the nucleus of the formula; the latter initiates a second $\mathrm{X}-\mathrm{C}-\mathrm{C}-\mathrm{C}-\mathrm{N}$-grouping; LSI), the fourth drug, differs from LAE only by a higher grade of alkylation on the primary amino group (di-ethyl). Hence we note that (1) a gradually higher grade of alkylation and (2) the presence of two "LSD-tails" ( $\mathrm{N}-\mathrm{C}-\mathrm{C}-\mathrm{C}-\mathrm{N}$ ) are among the structural concomitants of our basic drugs displaying an increasing selectivity for wool.

The next formula, methylene blue, shows us a substance of even higher affinity (i.e. absorption) for wool than LSD, namely $3.3 \times 10^{-2} \mathrm{mMoles} / \mathrm{gm}$. wool.

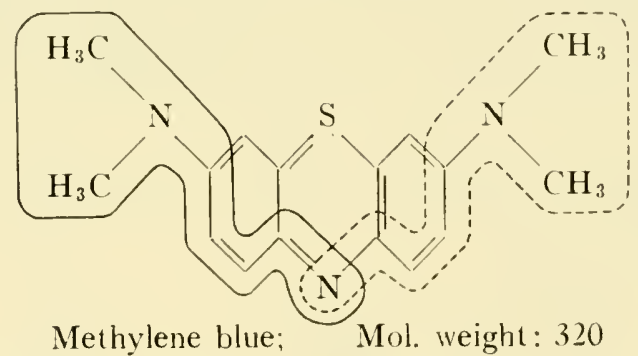

Here we observe two dialkylated "LSW-tails" each of which has 4 (' atoms (instead of 3, as in LAE and L.SD) between the primary amino group and the $\mathrm{N}$-atom of the ring.

Before considering some of the structural characteristics of SKF-501, which displayed the highest affinity for wool among all the compounds tested up to now in this study $\left(3.8 \times 10^{-2} \mathrm{mMoles} / \mathrm{gm}\right.$. wool $)$ let us draw attention to the findings of Harvey and Nickerson (1953), which show that compounds of the Dibenamine type, including SKF-501, might occur in the body in their ethylenimonium form. These authors also have shown that such a form exerts the same adrenergic blocking activity as the original configuration.

Increasing absorption (higher degree of specificity for wool protein) of basic (cation-active) drugs is accompanied (for further factors see Fischer and Larose, 1952b) by the following structural features: (a) increased grade of alkylation on the primary amino group, the latter which may be present as quaternary $N$ in viro, (b) increasing number of such amino-groups, (c) increasing number of 


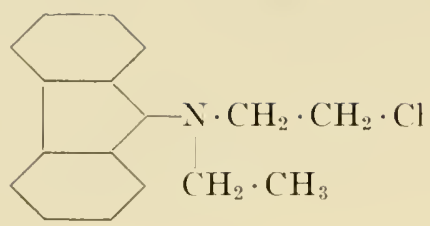

SKF-501

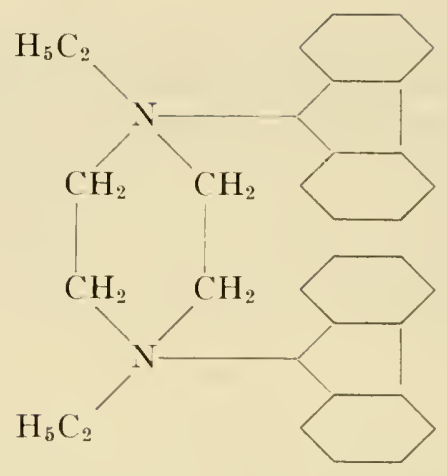

Ethylenimonium form of SKF-501

Mol. weight: 314

$\mathrm{C}$ atoms (up to 4) between the primary amino group(s) and the neighboring $\mathrm{N}$ of the nucleus, (d) the presence of two hidden "LSD-tails" and (e) a molecular weight around 320 appears to be characteristic of basic drugs displaying a high affinity for wool. Some of the aforementioned common structural features of LAE, LSD, methylene blue and SKF-501 remind us that among the phenazathionium compounds substitution in the amino group (alkylation) is accompanied by an increase in basicity; the latter characteristic, however, might be regarded only as one of the factors intluencing the affinity of our basic compounds for wool (Fischer and Larose, 1952b).

Since the following data, kindly supplied by the courtesy of Professor E. Rothlin (Basel, Switzerland), indicate that there is no increase in basicity if LAE is compared with LSD:

$$
\begin{aligned}
& \mathrm{pK}_{1} \text {, in } \mathrm{H}_{2} \mathrm{O} \text { of Mescaline...... } 4.25 \\
& \text { LAE. . . . . . . } 7.17 \\
& \text { LSD . . . . . . . } 7.12
\end{aligned}
$$

the higher affinity for wool of the latter appears to be due to other factors.

Numerous problems as well as questions arise in connection with the above speculations; for example, is the optically active ( -atom and its adjacent structural part, the

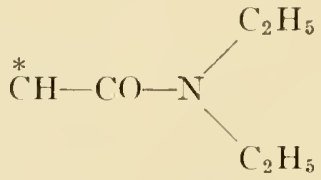

grouping involved in the highly specific action of LSD? Such questions remind us of the limitations of wool in this study, since (a) wool protein might be regarded only as a model of the protein component of the target organ(s) playing 
a part in the production of model psychoses by drugs, (b) affinity (sorption) of a drug for protein is only one of the factors considered up to now in this report.

The affinity for wool of Ergobasine(D-lysergic acid 1-propanol-amide-(2) and of Methylergobasine (D-lysergic acid $(+)$ butanolamide- $(2)$ ) was also measured and found to be $\approx 1.3 \mathrm{mMoles} \times 10^{-2} \mathrm{gm}$. wool. Conclusion: Among the D-lysergic acid derivatives LAE, LSD, Ergobasine and Methylergobasine, it is LSI), the biologically most potent compound, which displays the highest affinity for wool.

Finally let me digress in the direction of a very general speculation on biological activity. It might be that different levels of affinity for (wool) protein correspond to apparently different biological activities (as viewed by different specialists in biology). It seems that derivatives of a compound displaying high affinity for (e.g. wool) protein, exert various biological activities according to the degree of specificity they display toward the available receptors, some of them apparently containing a protein component structurally related to wool.

High affinity for wool of basic compounds seems to be associated with very potent biological activity. This activity is evidenced by the small dose of compounds required to exert their activity; e.g. the affinity of the biologically highly active chlorpromazin (Largactil) for wool is high, i.e. $5.3 \mathrm{mMoles} \times 10^{-2}$ per gm. wool. The low affinity of Atabrine for wool $\left(0.9 \mathrm{mMles} \times 10^{-2}\right.$ per $\mathrm{gm}$.) on the other hand may explain why it has to be administered in high dosage and for a prolonged period.
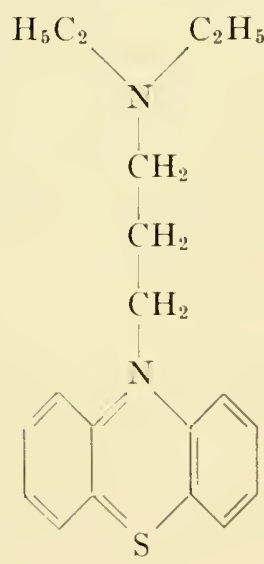

Chlorpromazine (Largactil)

Mol. weight : 318.5
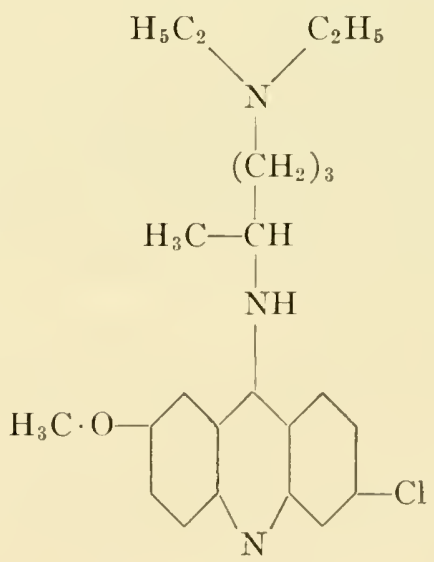

Atabrine (Mepacrine)

Mol. weight: 435.9

(1) Affinity to wool of a compound is influenced by certain structural as well as environmental factors. Some of the structural factors are: the size and the molecular weight of the compound; of course, it is also of impor- 
tance whether the compound is cationic (basic) or anionic (acidic) in nature (non-ionic compounds have no affinity for wool). Some of the environmental factors are: temperature, the presence and concentration of salts, $\mathrm{pH}$, the degree of dissociation (the higher the degree of dissociation, the lower the affinity of the compound for wool), the number and kind of reactive groups, such as () $\mathrm{H}, \mathrm{NH}_{2}$, elc.

(2) In addition, there is a finer structural configuration which will determine specific pharmacological action.

The affinity for wool of a substance appears to be a prerequisite for a certain type of biological activity; on the other hand, affinity for wool in itself does not imply a specific pharmacological activity.

\section{IV}

\section{Relation between Affinity for Wool of a Compound and its Biological Activity}

\section{ITool as a General Model for Cholinesteruse}

We have pointed out that there is a retation between the affinity of a chug for wool and the ability of the same drug to produce hallucinations.

In the following discussion we will consider only mescaline, LAE and LSI). It can be argued that mescaline in itself is not the active compound when administered to human volunteers but forms compounds similar to the LSD) or LAE structure. Such a hypothesis was recently postulated (Fischer, 1955) that the biosynthesis may be brought about through condensation of minute amounts of mescaline with nor-adrenalin or 5-hydroxytriptamine, both recently identified in the brain. Several data appear to support the above hypothesis that mescaline is transformed in rivo to a compound resembling LSD in its main structural features and having an affinity for wool, and that the observed physiopathological and psychopathological properties of mescaline are due to this LSD-like compound.

The prevention of a $80 \gamma$-LSD-caused psychosis, by the previous administration of a structurally related substance, a potential competitive inhibitor, was tried. Suitable compounds were selected from the phenothiazine series.

It is well known that methylene blue, a phenothiazine derivative, is capable of staining the ends of living nerves; that it is an analgesic, an antimalarial and an antiseptic. Phenothiazine, on the other hand, is an anthelmintic and the base substance of drugs with antihistaminic, fungicidal, adrenolytic and antiemetic activity. In addition these compounds cause a fall in body temperature, display a ganglionic blocking as well as a curarizing action and even some quinidine-like action. We found that methylene blue, $\mathrm{N}$-(2-diethylamino-n-propyl)phenothiazine (Parsitan), 3-chloro-10-(3-dimethylaminopropyl) phenothiazine (Largactil) and $\beta$-diethylaminoethyl-N-phenothiazine (Diparcol) display a 
gradually increasing affinity for wool $\left(3.3,4.8,5.5 . \mathrm{mMoles} \times 10^{-2}\right.$ per gm. wool respectively).

If, therefore, wool protein is a model of the neuro-receptors involved in the drug-induced experimental psychoses, then we might expect that this group of compounds will modify the LSD-caused psychosis perhaps by competitive inhibition. Preliminary experiments suggest that a gradual increase in affinity for wool of a compound might be associated with a more complete inhibition of the experimental psychosis. (For other factors, see Fischer, 1954).

A curvilinear relation was found to exist between the gradually increasing affinity for wool protein of the seven compounds (from mescaline to $\beta$-diethyl aminoethyl- $\mathrm{N}$-phenothiazine) and the logarithm of their relative toxicity $(0$, $1.26,2.54,2.9,3.35,3.43,3.43)$ towards 14 day old tadpoles of Yenopus levis (Fig. 2 and 3 ). Such a relation seems to indicate that apparently a similar type of receptor is involved in the production and inhibition of experimental psychoses. The increasing adrenergic blocking activity of LAE and LSD, as well as the gradually increasing adrenolytic action of the inhibitors mentioned can be evaluated as indirect evidence supporting our contention (Fischer, 1954: Fischer and Agnew, 1954).

Interpretation of our data can also be based on the hypothesis that "many

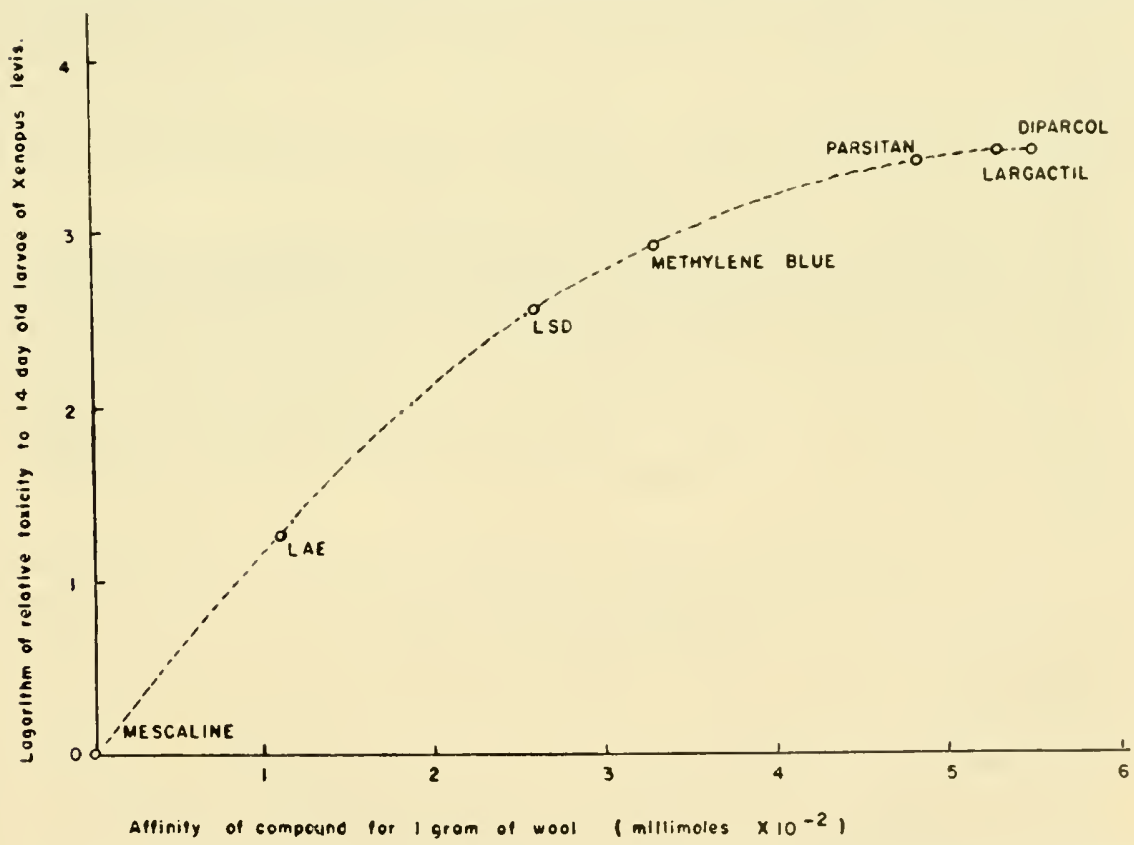

Fig. 2. Relation between the affinity for wool protein of seven compounds and the log. of their relative toxicity towards 14 day old tadpoles of Tenopus levis Daudin. 


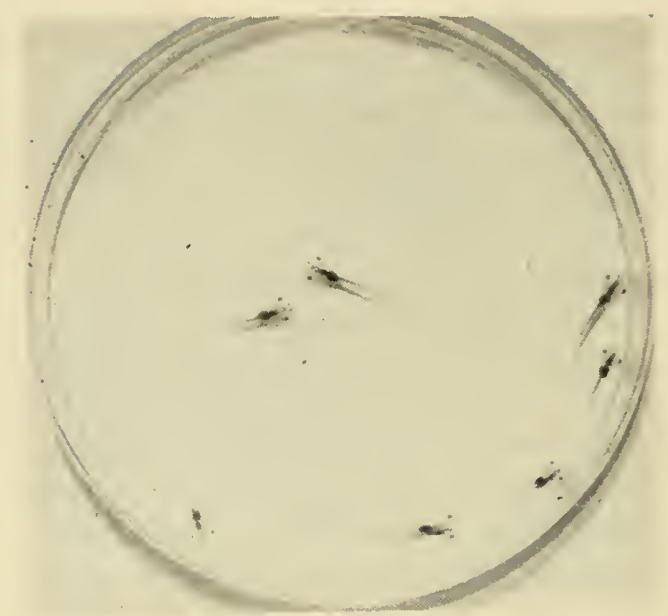

Fig. 3. Tadpoles of Xenopus lezis Daudin

biologically active substances may produce their specific effects by acting on the enzyme which inactivates them." (Zupancic, 1953). We might assume then that adsorption of our compounds on wool simulates the combination of the "cationic head"'s of active compounds with the anionic sides of the cholinesterase receptor. Some further data seem to support such an assumption. Janus green $\mathrm{B}$, a very potent inhibitor of cholinesterase, as well as certain quaternary ammonium compounds, exhibit an affinity for wool (especially the quaternaries display, the highest affinity, $7.0 \mathrm{mMol} . \times 10^{-2} \mathrm{gm}$. wool).

Since Zupancic (1953) and Smith et al, (1952) among others, claim that certain quaternaries, especially those antagonistic to tubocurarine, act by inhibiting cholinesterase, we suggest that wool protein in this study might be regarded as a model for the latter enzyme.

According to Bergmann and Shimoni (1953), cholinesterase behaves towards cations like a cation exchanger. Therefore, when the enzyme is built into the nerve membrane, the latter will acquire properties of a "permselective" membrane.

It is clear, state Bergmann and Shimoni (1953), that in the nerve membrane the resting potential is re-established long before the ion-exchange processes are finished. Therefore, the recovery process must be dependent on a chemical change in the membrane itself. A special mechanism must be available to recover the resting conditions through readjustment of the original concentration and activity of $\mathrm{H}^{+}$in the hydrated membrane phase. This could be done by an appropriate buffer, which would not prevent the original "acidification" required by the breakdown of selective permeability, but would react within a measurable, though very short time. The usual buffers would not serve this purpose. It is necessary to look for a fast chemical "buffering" reaction, cata- 
lyzed by an enzyme. In the nerve membrane a suitable system with a sufficiently high reaction velocity is represented by the acetylcholine-cholinesterase system. This is, so far, the fastest enzymic reaction known in animal cells, since one mole of enzyme can split $2.10^{7}$ moles of substrate per minute. According to Hestrin (1950), the equilibrium between the ester and its hydrolytic products

$$
\text { acetylcholine }+\mathrm{H}_{2} \mathrm{O} \rightleftarrows \text { acetic acid }+ \text { choline }
$$

is shifted to the left with decreasing $\mathrm{pH}$. Thus under more acid conditions more ester will be formed by the enzyme, whereas in a more alkaline environment hydrolysis will be favored. Taking into account the dissociation constant of acetic acid, $\mathrm{pK}_{\mathrm{a}}=4.7$, it is clear that the assumed buffer system will work best around $\mathrm{pH} 5$. We would like to mention in this context that our affinity for wool measurements have been carried out at $\mathrm{pH} 5-5.2$, i.e. very close to the iso-electric point of wool ( $\mathrm{pH} 4.9-5)$.

Since quaternary ammonium compounds display the highest affinity for wool protein and, on the other hand, we may regard wool protein as a model for cholinesterase, there are two points to be considered; first, the adsorption of the quaternary appears to interfere with the normal enzyme function either by neutralizing the anionic groups which may be necessary for enzyme action (this is the more likely explanation) or, secondly, by a steric interference preventing the action of the enzyme by covering too much of its surface.

\section{$\mathrm{V}$}

\section{Limitations when Using Affinity for Wool as an Indicator of Biological Activity}

In a series of adrenergic blocking agents not available for commercial use, and which were obtained by the McNeill Laboratories, Philadelphia, through the courtesy of Dr. Ansel P. Swain, we measured the affinity of the following six compounds for wool.

Affinity for $1 \mathrm{gm}$. of wool in mMoles $\times 10^{-2}$

\section{Compounds}

Mol. Weight

\section{3}

N, N-Diethyl 1-(3-indolylmethyl) isonipecotamide hydrochloride

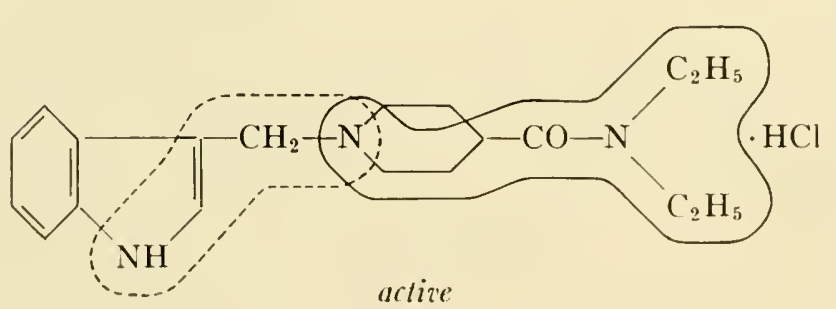

active
$\mathrm{C}_{19} \mathrm{H}_{27} \mathrm{~N}_{3} \mathrm{O}$.

$\mathrm{HCl}$

Mol. Wt.

349.9 
Affinity for $1 \mathrm{gm}$. of wool in mMoles $\times 10^{-2}$

\section{Compounds}

Mol. Weight

N, N-Diethyl 1-(2-phenylethyl) isoni recotamide hydrochloride

$\mathrm{C}_{18} \mathrm{H}_{29} \mathrm{ClN}_{2} \mathrm{O}$

Mol. Wt.

$32+.9$

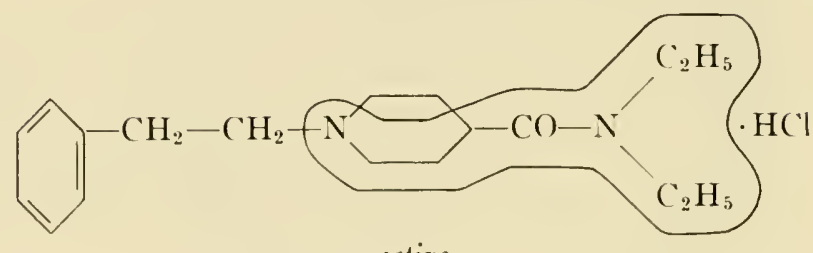

active

3.3 1-(3-Indolylmethyl) isonipecotamide hydro- $\mathrm{C}_{15} \mathrm{H}_{20} \mathrm{Cl} \mathrm{N}_{3} \mathrm{O}$ chloride

Mol. W't. 293.8

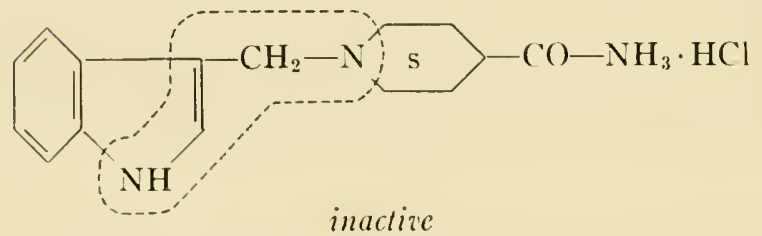

3.1 Methyl 1-(3-indolylmethyl) isonipecotate hydrochloride

$\mathrm{C}_{16} \mathrm{H}_{21} \mathrm{ClN}_{2} \mathrm{O}_{2}$

Mol. Wt. 308.8

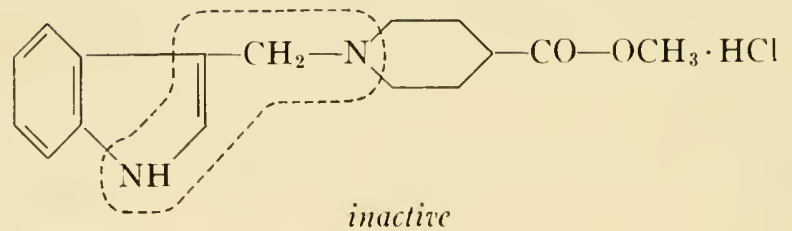

6.0 N-Benzyl-N-ethyl 1-(3-indolylmethyl) iso- $\mathrm{C}_{24} \mathrm{H}_{29} \mathrm{~N}_{3} \mathrm{O} \cdot \mathrm{HCl}$ nipecotamide hydrochíoride

Mol. Wt. +12.0

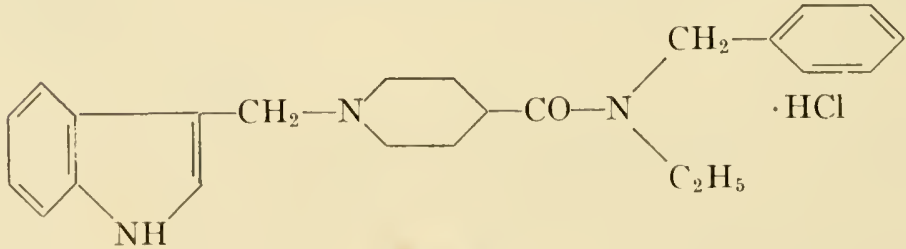

inaclive 
Affinity for $1 \mathrm{gm}$.

$\mathrm{x} 10^{-2}$

Compounds

Mol. Weight
N, N-Diisopropyl 1-(3-indolylmethyl) isonipecotamide hydrochloride
$\mathrm{C}_{21} \mathrm{H}_{32} \mathrm{ClN}_{3} \mathrm{O}$

Mol. Wt. 377.9

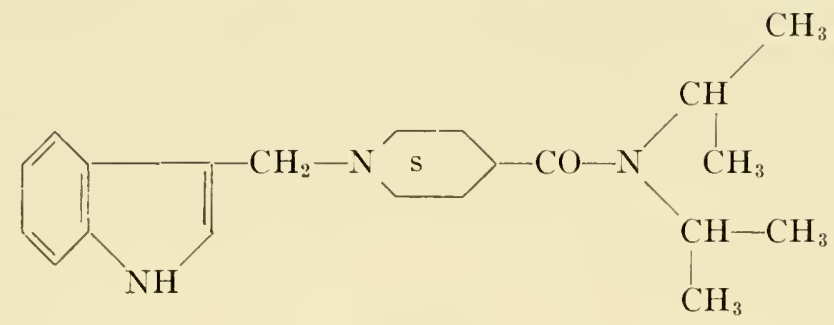

active

It is interesting to note that the first compound, which resembles most the LSD structure, displays a high affinity, namely $5.3 \mathrm{mMoles} \times 10^{-2} / \mathrm{gm}$. of wool and is a potent adrenergic blocking agent. The second compound is also an active one but with single - instead of two- "LSD-tails" due to the absence of the indole ring; its affinity for wool is, therefore, lower than that of the first compound. The third compound is inactive apparently because of its lack of the dialkylated "LSD-tail", causing, among others, a difference in basicity. The fourth compound seems to be inactive for the same reason as the third one. The fifth compound has the highest affinity for wool because of its large molecular weight (the largest in this series); the benzyl-group on the primary amino nitrogen, however, does not conform with that finer structure apparently needed for such compounds to display biological activity. The meaning of the "finer structure" is similar to that of Ehrlich's "key-hole" concept. The sixth compound has a high affinity for wool and is pharmacologically active; the dialkylated "LSD-tail," the indole nucleus with its $\mathrm{N}-\mathrm{C}-\mathrm{C}-\mathrm{CN}$-grouping are features fitting the requirements set up previously.

These results again illustrate our contention that affinity for wool of a compound does not imply a specific neuropharmacological activity if the finer structural configuration necessary for that activity is absent.

\section{VI}

\section{Interlude: Affinity for $\alpha$-keratin, a Clarification of Terms}

There are two types of bonds which might build cross-linkages between protein chains: (1) covalent bonds like disulfide, peptide, ester and ether bonds, or (2) the most common secondary bonds, e.g. hydrogen-van der Waals or saltbonds. Affinity of a substance to protein results in a binding by the aid of one or more bonds. A substance bound to protein by secondary bonds will display 
a low and sometimes reversible affinity, while covalent bonds bind the compound more firmly to protein (which means high affinity or sorption values). Of course, binding to wool occurs when factors affecting solubility, permeability and attraction of the compound are already operative.

In the last few years new data have been contributed to the discussion of the $\alpha$-helix configuration of polypeptide chains from proteins of the $\alpha$-keratin types. The suggestion of seven-strand protein cables in parallel orientation offered by Pauling and Corey (1953) resolves the difficulties involved in the previously surmised simple structure of the $\alpha$-keratin protein. A repeating unit of seven amino acid residues is believed to give rise to a seven-strand $\alpha$-cable about $30 \AA$ in diameter. The $x$-ray pattern of hair and other $\alpha$-keratin proteins can be accounted for by such a unit.

\section{VII}

\section{Coda}

For Serotonin the affinity value is low $=0.9 \mathrm{mMoles} \times 10^{-2} / \mathrm{gm}$. wool. This is in agreement with the results of Boyd, Rothlin, et al., (1955), who found LSD to be a potent antagonist of Serotonin when tested on the isolated uterus of castrated or estrogen pre-treated rats. Serotonin is also an inhibitor of both types of cholinesterase (Langemann, 1954).

Let us recall in this context that the affinity value of LSD is three times higher than that of Serotonin.

$\mathrm{HO}$
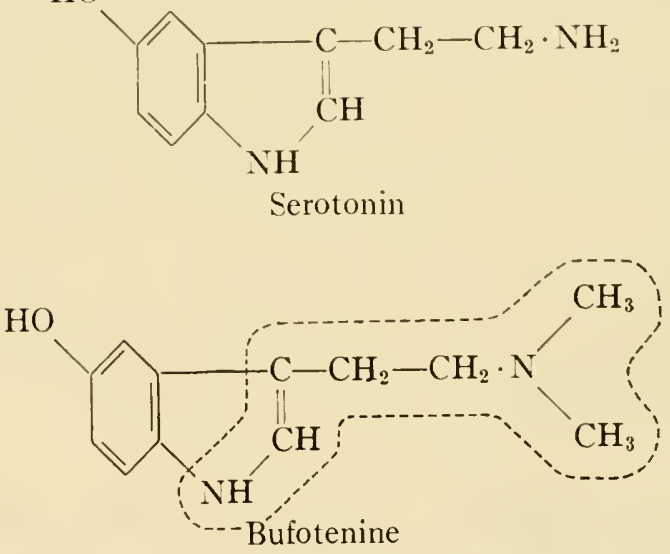

The affinity of Bufotenine, on the other hand, was found to be higher than that of LSD, i. e. 4 moles $\times 10^{-2} / \mathrm{gm}$. wool. This is indicative that Bufotenine is a more potent adrenergic blocking agent than $\mathrm{LSD}^{6}$.

${ }^{6}$ Since affinity of a compound for wool does not reveal anything about the rate of reaction of the compound, any prophecy referring to biological activity based on affinity of a compound to wool is a very general one; among other things it does not contain any information about the duration of that biological activity. 
We mentioned earlier that different levels of affinity for (wool) protein might correspond to apparently different biological activities. Therefore, we studied the affinity for wool of a series of compounds: antihistaminics, cholinesterase inhibitors, adrenergic blocking agents and quaternary ammonium compounds with sympathetic ganglionic blocking activity. Without going into too much detail, it can be stated that there is a gradually increasing affinity for wool of all these compounds displayed in the above-mentioned order. The lowest values are shown by Antistin, an antihistaminic of weak potency, and Panparnit, an agent used to relieve symptoms of Parkinson's disease. Then there is a slight increase when arriving at Pyribenzamine, as well as Prostigmine and Janus green (both cholinesterase inhibitors).

Benadryl $\left(3.5 \mathrm{mMoles} \times 10^{-2} / \mathrm{gm}\right.$. wool $)$ and Dibenamine show again an increase in their affinity for wool, followed by Priscol (2-benzylimidazolin) as well as by Regitin, 2-(N-p-tolyl-N-m-oxyphenyl-aminomethyl)-imidazolin, both adrenergic blocking agents. The latter is the more potent one and also has a higher affinity for wool than Priscol; the values are 1.7 and 4.3 mMoles $\times 10^{-2}$ / gm. wool respectively. Chlorpromazin (Largactil), Diparcol and Parsitan show affinity values around $5.3 \mathrm{~m}$ Moles $\times 10^{-2} / \mathrm{gm}$. wool and the two quaternaries, Desogen and Bradosol, phenoxy-ethyl-dimethyl-dodecyl-ammonium bromide. have the highest values recorded, namely $7 \mathrm{mMoles} \times 10^{-2} / \mathrm{gm}$. wool.

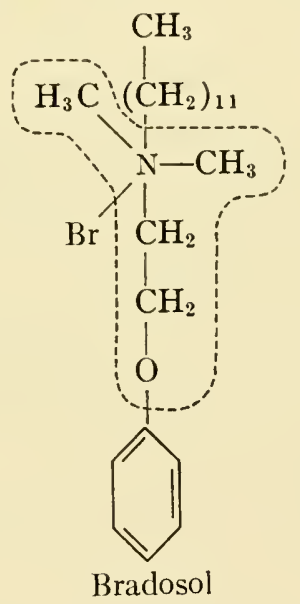

Thus wool is not only a model for a single enzyme but may also be regarded as a model in a wider sense for all enzymes.

The different levels of affinity for wool of certain compounds might imply that many compounds with high affinity display biological properties generally including also those associated with compounds of low affinity for wool. The implication may be drawn that there is a hierarchy in terms of biological activity which is associated with the degree of affinity for wool. In general, those compounds of a homologous series which show high affinity have also a wider range 
of biological activity than those with low affinity for wool. This would be in agreement with the observation that there are many substances each of which acts on more than one enzyme. Ergotamine, e.g. inhibits monoaminoxydase, diaminoxydase and cholinesterase.

Let me conclude with a quotation from Zupancic (1953): "The overlapping sensitivity of a few enzymes toward the same substance would be analogous to the retinal cone pigments with their overlapping but quantitatively different, absorption of the same monochromatic light."

\section{Summary}

Wool is used as an in vitro model of the proteinous surface of nervous receptors (enzymes) in zivo.

Affinity for wool appears to be among the characteristics of compounds acting on enzymes which are essential to maintain membrane polarization at rest and recovery after transmission.

Certain compounds, apparently those acting by competitive inhibition, are more firmly bound to wool (i.e. they display high affinity) than the compounds they displace.

The limitations of the wool-model are discussed and speculations forwarded to account for the overlapping sensitivity of a few enzymes toward the same substance.

\section{Acknowledgments}

I am indebted to Dr. Paul Larose, Head, Fibre Laboratory, Division of Pure Chemistry, National Research Laboratories, Ottawa, and to Dr. A. Hoffer, Chairman, Saskatchewan Committee on Schizophrenia Research, for their stimulating discussions and valuable advice.

The compounds used in these studies were generously supplied by the following pharmaceutical companies: Abbott Laboratories, Ciba, Geigy, HoffmanLaRoche, McNeill Laboratories, Parke Davis, Poulenc, Sandoz, Smith, Kline and French, Squibb, Upjohn, and Winthrop-Stearns. Figures and photographs are the work of Mr. Harry Wood, Regina.

These studies were supported by the Department of National Health and Welfare, Ottawa, Canada.

\section{References}

Bailey, P. 1950. Considérations sur l'organisation et les fonctions du cortex cérébral. Rev. Neurol. 82: 3-20.

Behringer, K. 1927. Der Mezkalinrausch. Springer. Berlin.

Bergmann, F. and A. Shimoni. 1953. The changes in the nerve membrane and the role of cholinesterase in the conductive process. Biochim. et Biophys. Acta. 10: $49-54$. 
Block, R. J. 1951. A comparative study on two samples of neurokeratin. Arch. Biochem. and Biophysics 31: 266-272.

Bovet, D., F. Bovet-Nitt, and V. G. Longo. 1952. Sympathomimetic properties of the ergotamine series-the pharmacological action of $\mathrm{N}$-(tetrahydro-2-naphthyl)$\mathrm{N}$-methyl- $\mathrm{N}^{\prime}$-ethyl-beta alaninamide (916 I.S.) and of amino-amido derivatives of 2 aminotetrahydronaphthalene. Rendiconti Inst. sup. Sanita 15: 925-952.

Bovet, D., F. Bovet-Nitti, and G. B. Marini-Bettolo. 1953. Abstr. Communications. 19th Intern. Physiological Congress, Montreal.

Boyd, E. S., E. Rothlin, J. F. Bonner, 1. H. Slater, and H. C. Hodge. 1955. Preliminary studies on the metabolism of lysergic acid diethylamide. J. Pharmacol. Exper. Therap. 113: 6-7.

Bradley, W. and G. C. Easty, 1951. Selective absorption of optical antipodes by proteins. J. Chem. Soc. 1951: 499-503.

Fischer, R. 1946. Selbstbeobachtungen im Mezkalin-Rausch. Schweiz. Zeitschr. f. Psychologie. 5: 308-313.

Fischer, R. 1953. The selectivity of the gram stain for keratins. Exper. 9: 20-21.

Fischer, R. 1954. Factors involved in drug produced model psychoses. J. Ment. Sci. 100: 623-631.

Fischer, R. 1955. Possible biosynthesis of D-lysergic acid diethylamide-like compounds from mescaline. Exper. 11: 162-163.

Fischer, R. and N. Agnew. 1954. On drug-produced experimental psychoses. Naturwissenschaften. $41: 431-432$.

Fischer, R., F. Georgi, and R. Weber. 1951. Psychophysische Korrelationen; Modellversuche zum Schizophrenieproblem; Lysergsäurediäthylamid und Mezcalin. Schweiz. med. Wchschr. 81: 817-819.

Fischer, R., A. Hoelle, and S. Seidenberg. 1951. Voraussage der bakteriziclen Wirkung von Substanzen durch Bestimmung ihrer Affinität zu Wolle. Helv. Chim. Acta 37: $210-222$.

Fischer, R. and P. Larose. 1952a. Contribution on the behavior and structure of the cytoplasmic membrane of bacteria. Can. J. Med. Sci. 30: 86-105.

Fischer, R. and P. Larose. 1952b. Mechanism of Gram stain reversal. J. Bact. 6t: $435-441$.

Fischer, R. and Seidenberg, S. 1951. Homologous mechanism of bactericidal action and Gram-staining. Science 11: 265-266.

Greiber, M. F. 1947. Psychoses associated with administration of atabrine. Am. J. Psychiat. 10\%: 306-314.

Halpern, L., M. Streifler, and L. Laszlo. 1953. Electrical activity of brain in case of atabrine psychosis. Am. J. Psychiat. 110: 366-369.

Harrer, G. and H. J. Urban. 1953. Psychosen als Komplikation bei Eingriffen am Sympathicus. Nervenarzt. 24: 63-64.

Harvey, S. C. and M. Nickerson. 1953. Chemical transformations of dibenamine and dibenzyline and biological activity. J. Pharmacol. Exper. Therap. 109: 328-339.

Hestrin, S. 1950. Acylation reactions mediated by purified acetylcholine esterase II. Biochim. et Biophys. Acta 4: 310-321.

Hoobler, S. W. and A. S. Dontas. 1953. Drug treatment of hypertension. Pharmacol. Rev. 5: $135-174$.

langemann, II. 1954. 5-Hydroxytryptamine as an anticholinesterase. Hely. Physiol. et Pharmacol. Acta 12: C28.

Larose, P. and R. Fischer. 1952. A test for bactericidal activity in which bacteria are replaced by wool. Research (London) 5: 419-423. 
Larose, P. and R. Fischer. 1953a. Reversal of gram-staining behavior. Science 117: $449-451$.

Larose, P. and R. Fischer. 1953b. Über die Affinitat quaternarer Ammoniumbasen zu Wolle und zu Bakterien. Schweiz. Ztschr. allg. Path. 16: 97-105.

Liddell, D. W. and H. Weil-Malherbe. 1953. Effects of methedrine and of lysergic acid diethylamide on mental processes and on blood adrenaline level. J. Neurol. Neurosurg. Psychiat. 16: 7-13.

Mayer-Gross, W., W. McAdam, and J. W. Walker. 1951. Psychological and biochemical effects of lysergic acid diethylamide. Nature 168: 827-828.

Mayer-Gross, W., W. McAdam, and J. W. Walker. 1953. Further observations on effects of lysergic acid diethylamide. J. Mental Sci. 99: 804-808.

Nickerson, M., J. W. Henry, and G. M. Nomaguchi. 1953. Blockade of responses to epinephrine and norepinephrine by dibenamine congeners. J. Pharmacol. Exper. Therap. 107: 300-309.

Pauling, L. and R. B. Corey. 1953. Compound helical configurations of polypeptide chains; structure of proteins of the alpha-keratin type. Nature 171: 59-61.

Rothlin, E. Private communication.

Schmitt, F. O., R. S. Bear, C. E. Hall, and M. A. Jakus. 1947. Electron microscope and $\mathrm{x}$-ray diffraction studies of muscle structure. Ann. N. Y. Acad. Sc. 47: 799-813.

Smith, C. M. et al. 1952. Mode of action of antagonists to curare. J. Pharmacol. Exper. Therap. 105: 391-399.

Solms, H. 1953. Lysergsäure-äthylamid (lae), ein neues, stark sedative wirkendes Psychoticum aus dem Mutterkorn; vorläufige Mitteilung. Schweiz. Med Wchnschr. 83: 356-360.

Stoll, W. A. 1947. Lysergsäure-diäthylamid, ein Phantastikum aus der Mutterkorngruppe. Schweiz. Arch. f. Neurol. u. Psychiat. 60: 279-323.

Szatmari, A. Private communication.

Walther-Büel, H. 1949. Die Dibenaminpsychose. Monatsschr. Psychiatr. a. Neurol. 118: 129-160.

Zupancic, A. O. 1953. Mode of action of acetylcholine. Theory extended to hypothesis on mode of action of other biologically active substances. Acta Physiol. Scand. 29: $63-71$.

ChAIRMAN GERARD: Thank you very much Dr. Fischer. I am sure there will be much discussion of your woolly experiments and woolly suggestions.

DR. BLum: Have you investigated any other proteins which would be of, say, non-epidermal origin?

Dr. Fischer: We measured the affinity of compounds to epidermal keratin as well as to proteins of non-epidermal origin, such as egg albumin, casein and insulin; the results are different from and bear no relation to those obtained when using wool protein.

Dr. Robert Resnick (National Institute of Mental Health): It seemed to me that it might be fortuitous to conduct experiments with one and try to extrapolate to other products when they might be similar in composition.

Dr. Fiscier: As to the similarity in composition of different types of kera- 
tins, allow me to refer to the following tabulation:

\begin{tabular}{|c|c|c|c|}
\hline \multicolumn{2}{|l|}{ Molecular Ratio } & \multicolumn{2}{|c|}{ Type of Keratin } \\
\hline Histidine:Ly sine: Irginine & $1: 2: 2$ & Neurokeratin & \\
\hline Histidine: Lysine: Arginine & $1: 4: 4$ & epidermal keratin $\int$ & \\
\hline Histidine: Lysine: Irginine & $1: 4: 12$ & $\alpha$-keratin & hard \\
\hline
\end{tabular}

Dr. Jordi Folch-Pi (McLean Hospital, Waverly, Mass.): Dr. Fischer, why did you not do that with neurokeratin which can be prepared quite easily?

Dr. Fischer: I have no access to it. I am very sorry.

Dr. Folch-PI: Prepare it.

Dr. Fischer: Wool was chosen for other reasons. In 1946-47 I began to use wool as a model for bacteria. I have found since that there is a relationship between the affinity of water soluble substances for wool and their bactericidal activity against vegetative cells of certain bacteria. A further relationship between bactericidal activity and Gram-staining behavior was then expressed quantitatively and indicated, among other things, that the similarity in behavior between gram-negative, gram-variable, and gram-positive bacteria on the one hand and intact wool, medium-alkali-degraded wool and highly-alkalidegraded wool on the other, apparently is caused by some structural feature common to the wool and the bacterial cytoplasmic membrane.

Dr. Folch-PI: Neurokeratin is very easy to prepare and the way to prepare it is very similar to the way in which wool is normally treated. It is defatted and treated with alkali and then you do not have to spend hours justifying why you think that the neurons are covered by a sweater.

Dr. Fischer: If one treats wool with alkali then one obtains alkali-clegraded wool with broken disulfide linkages and free aldehyde groups, etc.; we have found the affinity of compounds towards such wools quite different from that which they display to intact (undamaged) wool. In our experiments we use defatted wool which has been equilibrated with diluted acetic acid at $\mathrm{pH} 4.9$, the isoelectric point of the wool.

Dr. Folcu-Pr: What we call neurokeratin, the composition which you are quoting, has been obtained by that method. Therefore, any similarity that you claim between your undenatured wool and the neurokeratin would not be supported by the very thesis you are putting forth. You could prepare it by milder methods.

Dr. Fischer: Would it be the same?

Dr. Folcr-Pr: You could skip the alkali because neurokeratin is not considered a keratin chemically. In a recent paper it was pointed out that while both might have similar, if not identical, amino acid composition there are certain differences such as the location of groups.

Dr. Fischer: There is an unusually high amount of sulphur present in wool 
keratin, around $80 \%$ or more of which is in the form of cystine. We were interested therefore in determining the possible influence of this sulphur on the affinity of compounds for wool.

I performed some experiments with what we call the spectrophotometric method; that is, we measured the absolute affinity of a compound to wool and compared it then with the relative affinity obtained by the weight increase method just mentioned. Using crystal violet as test substance, the determination of the absolute affinity to intact wool showed us that the wool does not take up any dyestuff at all: experimental conditions: $24^{\circ} \mathrm{C}$., $\mathrm{pH}=6.3,0.2 . \mathrm{gm}$. dyestuff, $50 \mathrm{cc}$. of water, $1 \mathrm{gm}$. of wool, $10 \mathrm{~min}$. However, $1 \mathrm{gm}$. of wool which has been pretreated with $0.15 \mathrm{~N} \mathrm{Na}_{2} \mathrm{CO}_{3}$ at $80^{\circ} \mathrm{C}$. for 3 hours, (H.D. $=$ highly degraded wool) took up $24 \gamma$ of crystal violet, that is $0.15 \gamma$ per mg. H.D. woolnitrogen.

If we block the SH-groups of H.D.-wool by a "Salyrgan"-treatment, this wool will take up only $0.08 \gamma$ crystal violet per mg. H.D. wool-nitrogen under otherwise similar experimental conditions. However, we found afterwards that any treatment of H.D.-wool which diminishes its base-attracting groups will diminish its affinity for cationic compounds.

Dr. Morales: I should think that if binding is unaffected by blocking the disulphide linkages then you are obligated to try this on another protein since that certainly was one thing that was characteristic. I would think that myosin or something of the same fibrous series would be it.

The things that I started to ask you were these. Experimentally, how do you measure the binding and, secondly, your explanation of how these things bind escaped me-I mean, what kind of forces you thought they were.

DR. Fischer: The affinity in those experiments which give relative values is measured on a weight increase basis. One gm. of wool is brought to constant weight-accuracy up to the fourth decimal point-over calcium chloride. A "Grammatic" balance and weighing times of 20 seconds are used for each wool sample under standardized conditions. There are always 2 "air"-controls and one "water"-control in a series of 5 wool samples. At $90^{\circ} \mathrm{C}$. and in 10 minutes the affinity to wool of $0.2 \mathrm{gm}$. of substance is measured in $50 \mathrm{cc}$. of distilled water at $\mathrm{pH} 5.2$, which is very near to the $\mathrm{pH}$ of the wool. Then the wools are rinsed three times with water at room temperature, pre-dried in an oven at $50-60^{\circ} \mathrm{C}$. for about 20 minutes and then again placed in the desiccator. After $3-4$ days, when they reach constant weight, the affinity is calculated from the weight increase.

If we measure the absolute affinity of a compound, then we submerge one gram of wool into a solution of $0.2 \mathrm{gm}$. of substance in $50 \mathrm{cc}$. of water and soak the wool there for 10 minutes. There is no rinsing with water but direct spectrophotometric determination of the difference in concentration of the "dyebath" prior and after the experiment. 
As to the second question about which bonds are involved in the binding of substances to wool, I cannot tell you which type of bond is actually involverl in each particular case. This is a very complex problem.

Dr. Morales: The reason I ask you is that it sounds like awfully tight binding. I was going to question whether you thought these relatively loose bonds would account for such tight binding.

Dr. Fiscner: The fact is that theoretically you can desorb every substance completely from wool. The question is only one of the appropriate experimental conditions, i.e. how long a time, what $\mathrm{pH}$ and what temperature, etc. you use. Our affinity values, I believe, represent equilibrium values under the stated experimental conditions.

Dr. Amadeo S. Marrazzi (Army Chemical Center): I suppose if it is justifiable to have a model psychosis it is justifiable to have a model receptor. I would like to shift the discussion from a purely chemical to a functional one as well and I would like to point out two facts that seem to me to need explanation. On the basis of affinity of chlorpromazine for the wool you predict an antagonism to the psychosis; whereas, in fact, the only antagonism is to one aspect of it. Hallucinations still go on so there is not a true pharmacological antagonism. In other instances with serotonin where you would have very low affinity, Dr. Hart and I have been using synaptic inhibition as an index and that, of course, requires affinity. In our experiments serotonin is more active despite its low affinity on your model.

Dr. Fiscier: Thank you, Dr. Marrazzi. I think chlorpromazine is very likely to act on quite a few different receptors; if there is an antagonism between chlorpromazine and certain symptoms of an LSD caused model psychosis, then this antagonism is likely to be due to the interaction of only one or a few receptors with the drug. I am told by the psychiatrists and have seen myself that a patient who sees the devil and is frightened by him prior to chlorpromazine, sees the devil even after chlorpromazine, although he is not frightened anymore. 


\title{
Glucose and Oxygen Utilization in Sympa- thetic Ganglia. I. Effects of Anesthetics. II. Substrates for Oxidation at Rest and in Activity
}

\author{
Martin G. Larrabee and Paul Horowicz'
}

The Thomas C. Jenkins Department of Biophysics, Johns Hopkins University, Baltimore, $M d$.

\section{Effects of Anesthetics}

$\mathrm{T}$ HE PURPOSE OF THE FIRST PART of this paper is to discuss some previously published observations on the effects of anesthetics on mammalian sympathetic ganglia, in relation to the hypothesis that the functional changes in neurons caused by anesthetics are secondary to metabolic disturbances. These investigations of effects of anesthetics on the synaptic system in sympathetic ganglia were undertaken because it did not seem possible to deduce with assurance the causal relationships between metabolic and functional effects observed in the brain (Butler, 1950; Larrabee and Bronk, 1952). It was thought that observations on a simpler neuronal system might yield helpful clues.

Sympathetic ganglia of mammals are suitable for such studies, since (a) some anesthetics have a selective action on synaptic transmission within these ganglia, in the sense that transmission is blocked by considerably lower concentrations than those required for block of conduction along the axons leading to and from these synapses (Larrabee and Posternak, 1952), and (b) some anesthetics interfere with ganglionic transmission in concentrations as low as those employed to depress the central nervous system during general anesthesia (Larrabee and Holaday, 1952). Thus a relatively simple preparation is available which is adequately sensitive to anesthetics, contains nerve cells that remain at rest until deliberately activated, and survives for many hours after excision when kept at body temperature in appropriate bathing solutions. Accordingly some of the difficulties encountered in interpreting experiments

Previously published investigations cited in Section I of this paper, and some observations preliminary to experiments reported in Section II, were aided by a grant from the Supreme Council, Scottish Rite Masons, for research under the direction of D. W. Bronk. New investigations presented in Section II were supported in part by a research grant (No. B-702) from the National Institute of Neurological Diseases and Blindness of the National Institutes of Health, U. S. Public Health Service.

${ }^{1}$ Fellow of the National Science Foundation, 1952-1955. 
TABLE I

Metabolic effects of anesthetics in concentrations causing $50 \%$ reduction in postganglionic response

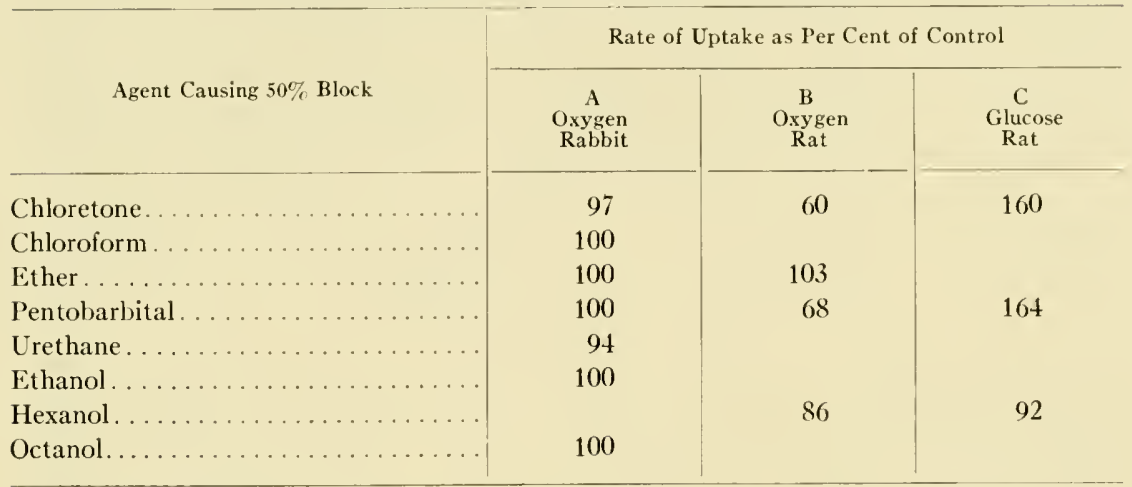

A. From Larrabee, Ramos and Bülbring (1952).

B. and C. From Edwards and Larrabee (1955).

on brain tissue can be avoided. Advantages are that observations can be made in various controlled states of activity, the response to stimulation can be measured accurately by recording from the preganglionic and postganglionic nerves, and the effects of anesthetics on metabolic exchange and nervous function can be observed simultaneously or at least under similar conditions.

\section{Anesthetics on Metabolism of Ganglia at Rest}

Our first metabolic experiments in connection with anesthetics were concerned with the resting rate of oxygen uptake by superior cervical ganglia excised from rabbits. Most, if not all, of the anesthetics employed depressed the resting rate of oxygen consumption when present in sufficient concentrations. This is in agreement with the results of many investigators on brain (Quastel, 1952). More significant, however, was the finding that resting oxygen uptake was not significantly disturbed at the minimal concentrations required for impairment of ganglionic transmission (Table I, Column A).

Results different from those on rabbits were obtained on ganglia excised from rats (Edwards and Larrabee, 1955). Here it was found that oxygen uptake was definitely slowed by at least three agents in concentrations causing $50 \%$ block of transmission (Table I, Column B). Moreover there was additional evidence of a metabolic disturbance, since the rate of glucose uptake was found to be considerably increased by certain agents, as shown in Column C of Table I. This is in agreement with results of several studies on brain (Greig, 1947; McIlwain, 1953; Rosenberg, Buchel, Etting and Levy, 1950; Webb and Elliott, 1951). 
Although a species was thus found in which resting metabolism of ganglia was disturbed by significantly low concentrations of some agents, a number of reasons suggested caution in concluiding that the metabolic changes were necessarily the cause of the loss of function. One reason was that still greater metabolic changes than these could be caused by a substance such as azide, at least in the rate of oxygen consumption, without blocking transmission. Secondly, even in the rat, one circumstance was found where transmission was slightly but significantly impaired without change in uptake either of oxygen or of glucose; this was during the application of $0.5 \mathrm{mM}$ chloretone. In another case, that of ether, oxygen uptake was not significantly changed even with $50 \%$ block of transmission; possible effects of this agent on glucose uptake have not yet been studied. Finally, the marked contrast between ganglia from rats and rabbits suggests that the coincidence of functional and metabolic changes, found only in rats, may not signify a causal relation, but may merely indicate that in this species two independent effects happen to be produced in the same range of concentrations. These results thus yield no compelling evidence that the basis of anesthetic action lies in effects on the resting component of metabolism.

\section{Anesthetics on Metabolism in Actize Ganglia}

Several lines of evidence indicate that conduction and discharge of nerve impulses are associated with some chemical reactions which occur at a much slower rate or not at all in resting neurons. We have recently found, for example, that significant amounts of endogenous substrates are oxidized during activity in excised sympathetic ganglia, whereas the oxygen consumed at rest can be almost entirely accounted for by oxidation of exogenous glucose. (See Part II of this paper.) These findings are in agreement with observations of others on peripheral nerve trunks (Holmes et al., 1930; Mullins, 1953). Moreover it has been reported that the activity increment in oxygen consumption by nerves and muscles of frogs is depressed by lower concentrations of azide than is the resting rate of oxygen uptake; this may also be interpreted by assuming that different reactions are involved in the activity and resting components of metabolism (Brink et al., 1952; Doty and Gerard, 1950; Stannard, 1939).

It is therefore possible that an anesthetic, in appropriate concentrations, might interfere with chemical reactions which are of importance only during activity. This might explain, for example, why oxygen consumption is more readily depressed by anesthetics when tissues are submitted to appropriate electrical stimulation than when no such stimulation is applied. This has been observed in frog nerve (Brink et al., 1952), in mammalian sympathetic ganglia (Larrabee, Ramos and Bülbring, 1952), and in slices of cerebral cortex (McIlwain, 1953). It might also explain why electrical responses to repetitive excitation are more affected by a given concentration of anesthetic than is the response to a single stimulus in ganglia (Larrabee and Posternak, 1952), and brain (Forbes 


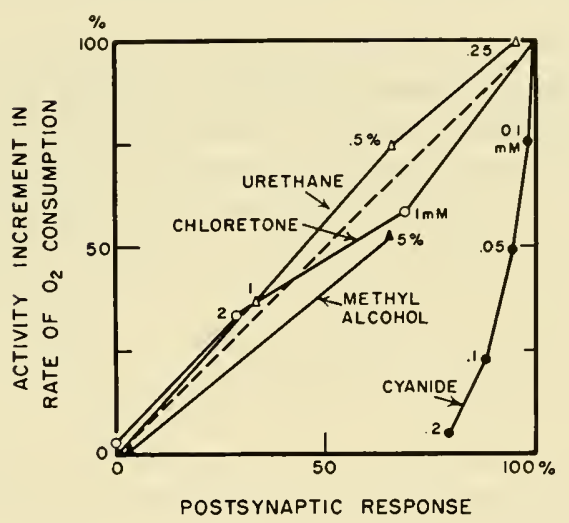

F1G. 1. Effects of anesthetics and of cyanide on the activity increment in rate of oxygen consumption (ordinates) and on the height of the postganglionic action potential (abscissae) in excised superior cervical ganglia (rabbit). The preganglionic nerve was stimulated supramaximally about 10 times per second. Both variables are expressed in percent of their values in the absence of experimental substances. (Larrabee, Ramos, and Bülbring, 1952).

et al., 1949; Jarcho, 1949; Marshall, 1941; Marshall, Woolsey and Bard, 1941). Such selective effects on the activity component of metabolism, if indeed they do occur, might be especially significant in the action of anesthetics on the functional capacity of the brain, where normal physiological properties are associated with a background of continual spontaneous neuronal activity.

A set of experiments, designed to compare the effects of anesthetics on the activity increment in oxygen uptake with the effects on the neuronal activity itself, are summarized in Fig. 1. Here the number of impulses discharged by a ganglion during repetitive activity is measured by the height of the postganglionic action potential. This is depressed in almost exactly the same proportion as the increment in oxygen consumption, by each concentration of the anesthetics tested. This raises the question whether the first step in action of the anesthetics is interference with oxidations, or whether the activity is depressed by some other mechanism, the decline in rate of oxygen uptake then resulting as cellular activity subsides towards the resting level. This experiment is probably best interpreted on the second basis (i.e., as evidence against a primary disturbance in oxygen metabolism), since there generally seems to be a factor of safety in the chemical reaction systems; moderate oxidative disturbances do not in general lead to loss of function, at least not with the rapidity revealed in effects of anesthetics. The existence of a factor of safety is confirmed in Figure 1 by the application of cyanide to rabbit ganglia. In this case the activity increment in oxygen consumption was almost completely abolished with relatively little effect on the activity itself.

The experiments on ganglia just described illustrate a problem in interpreta- 
tion which also pertains to experiments on brain in situ, where the spontaneous activity of the neurons presumably provides a background of activity metabolism. Therefore depression of oxygen consumption by anesthetics is perhaps a secondary effect of depression of the spontaneous activity. A more definite conclusion might be reached if enough data were available to permit application of a criterion employed by Lewis and McIlwain (1954) in interpreting effects of other agents on electrically stimulated cerebral cortex. According to this criterion a blocking agent which acts non-metabolically would be expected to alter the various aspects of metabolism in the same way as withdrawal of excitation. A metabolic poison, on the other hand, should cause a disproportionately large change in that portion of the metabolism most closely related to its site of action. Unfortunately definitive experiments along these lines have not yet been performed on ganglia.

\section{Selective Action of Anesthetics in Relation to Metabolic Hypotheses}

An example of selective block of synaptic transmission through an excised rat ganglion is shown in Figure 2. The middle and lower records on the left show how pentobarbital first reduced the height of the postganglionic response (middle record) and then at high concentrations abolished it (lower record), with little or no change in impulse conduction along the preganglionic nerve. In other words, this agent selectively blocked synaptic transmission. On the
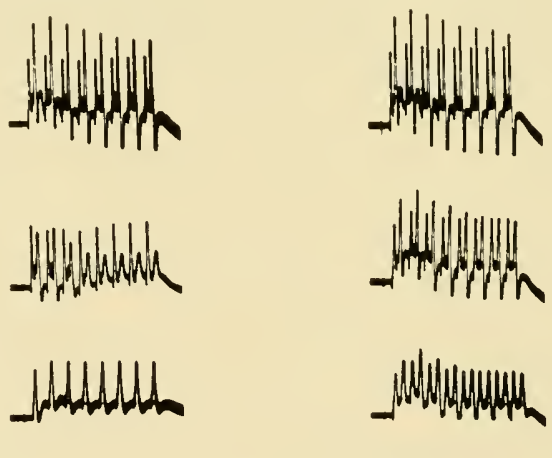

FIG. 2. Left: Effects of pentobarbital on transmission through a superior cervical ganglion excised from a rat. Right: Effects of urethane. In each record eight supramaximal stimuli were applied in succession to the preganglionic nerve at a frequency of 30 per second. Each stimulus evoked two upward deflections. Recording conditions were such that in the control (upper) records the first (smaller) deflection of each pair signalled the conduction of impulses towards the ganglion along the preganglionic nerve and the second (larger) deflection signalled the discharge of impulses away from the ganglion over the postganglionic nerve. Middle and lower records show effects of 0.3 and $1.0 \mathrm{mM}$ pentobarbital (on left) and 56 and $112 \mathrm{mM}$ urethane (on right). (Modified from Larrabee and Posternak, 1952). 
TABLE II

Possible bases of selective action

1. Differences in factors of safety in nerve conduction and synaptic transmission.

2. Non-uniform distribution of anesthetic due to differences in:

Solubility

Permeability

Intracellular destruction

3. Metabolic differences between nerve fibers and synaptic structures.

other hand, urethane, whose effects are shown in the right-hand column of Fig. 2, depressed pre- and postganglionic responses approximately equally, thus lacking selective effect on the synapses in this preparation. Some other anesthetics were found to be intermediate to the two used for illustrations, the degree of selective action tending to increase with molecular weight among the several agents tested (Larrabee and Posternak, 1952).

In attempting to explain selective action, it is apparent that the well known large factor of safety in nerve conduction as compared to synaptic transmission may be of major importance (Table II). It is difficult, however, although perhaps not impossible, to account on this basis for variations in intensity of selective action exhibited by different substances at the same synapses (Larrabee and Posternak, 1952). A second way of explaining selective action is to assume that the agents appear at higher concentrations in critical synaptic structures, such as the presynaptic endings or the postsynaptic cell body, than they do in axons. This could result from localized differences in solubility, in permeability, or possibly in rates of intracellular destruction of the anesthetic agent. A third proposal for explaining selective action is based on a hypothetical difference in the biochemistry of synaptic and axonal structures. For example, we might suppose that particular enzymes affected by anesthetics are either less abundant or more essential in certain synaptic parts of cells than they are in axons, so that a given concentration of inhibitor could cause the greatest derangements of cellular metabolism in synaptic regions.

Metabolic theories of anesthetic mechanisms can explain selective action on any of these several bases. In accordance with any such assumptions it seems reasonable to suppose that selective effects might also be produced by metabolic inhibitors other than anesthetics. Accordingly, we have tested the various substances listed in Table III, chosen to represent a variety of metabolic sites of action, as indicated in the last column (Larrabee and Bronk, 1952). For each agent the concentration was determined at which the height of the preganglionic response to supramaximal preganglionic stimulation was reduced to $80 \%$ of its control value. At this concentration there always remained a sizable postganglionic response, as indicated in the table, with the possible exception of a 
TABLE III

Effects of metabolic inhibitors on nerve conduction and on synaptic transmission in superior cervical ganglia excised from rats

(Observations by N. A. Coulter and J. Dempsher)

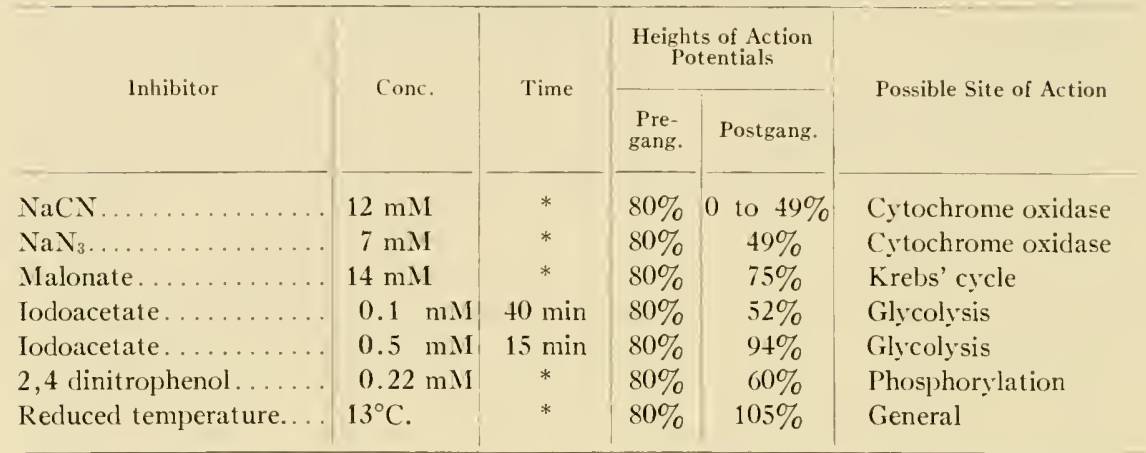

* Heights of action potentials became constant at indicated levels during application of inhibitor.

This table reproduced from Larrabee and Bronk (1952).

few experiments with cyanide. Thus none of these metabolic inhibitors produced a selective action comparable to that of pentobarbital, for example, which completely blocked transmission through a ganglion in concentrations causing no depression at all in the response of the preganglionic nerve.

\section{Conclusion}

In the foregoing discussion, we have touched on only a few of the types of metabolic studies that have been made by other investigators in relation to anesthetics. Innumerable such investigations, some on excised tissues and others on tissues in situ, have revealed many significant effects and have led to many interesting theories of anesthetic mechanisms. But as the story now stands, real difficulties in deciphering causal relations in anesthetic action are found in the experimental approaches which have been applied to the metabolic aspects of the problem.

In our own experiments, measurements of metabolism and tests with inhibitors have produced no compelling support for the metabolic hypothesis of anesthetic action. Neither has this interesting and important theory been controverted. In this connection it may be noted that a substance becomes classified as an anesthetic when it has been found on an entirely empirical basis to produce a certain general category of effects in the intact organism. Therefore it is possible that chemical agents, thus selected with attributes desirable for inducing anesthesia, may all be ones which cause metabolic disturbances not revealed in changes of oxygen uptake and not matched by any of the inhibitors listed in Table III. 
Moreover, the very fact of selective action warns against discarding metabolic theories merely because indisputably direct metabolic effects of anesthetics in appropriate concentrations have not yet been detected. Selective action means effects limited to particular regions. If important metabolic disturbances were localized in restricted critical structures, the changes might well be undetectable amidst the total metabolism of a whole neuronal aggregate.

\section{Substrates for Oxidation at Rest and in Activity}

The preceding descriptions illustrate some types of experiments that can be performed on sympathetic ganglia, taking advantage of survival of function after excision and the possibility of regulating and measuring the amount of neuronal activity. It is obvious that more could be learned if the number of ways of measuring metabolism were increased. Not only would investigations concerning anesthetics be aided, but so would knowledge of the normal metabolism of nerve cells, including the changes in chemical reactions accompanying the shift from rest to activity. Moreover, we have found that these ganglia and their related nerves are dependent on an external supply of glucose, in contrast to previous experience with excised peripheral nerve trunks. Thus here may be a particularly favorable opportunity for studying the use of this important substrate.

Accordingly, we have recently devoted considerable effort to developing and improving methods for measuring metabolism in small ganglia. The rate of oxygen uptake can now be measured in absolute terms, instead of only relative to control states as formerly, while stimulating electrically and recording action potentials. This is done under sterile conditions. Measurements of glucose uptake and lactate production have also been obtained, both at rest and during activity. In addition we are now measuring the rate of ammonia production, as a clue to amino acid metabolism.

\section{Methods}

Superior cervical ganglia, together with portions of the preganglionic and postganglionic nerves, were excised from anesthetized rats. Wet weights of these preparations were about $1 \mathrm{mg}$. The preparations were bathed at $35^{\circ}$ to $37^{\circ} \mathrm{C}$ in a bicarbonate-buffered solution previously described (Larrabee and Bronk, 1952, Table I, solution D). Under these conditions capacity for axonal conduction and synaptic transmission survived for 12 to 24 hours.

\section{Oxygen Consumplion}

The respirometer used in measuring rate of oxygen consumption was based on the continuous flow principle described by Carlson, Brink and Bronk (1950). In this method a suitably polarized platinum electrode (Davies and Brink, 


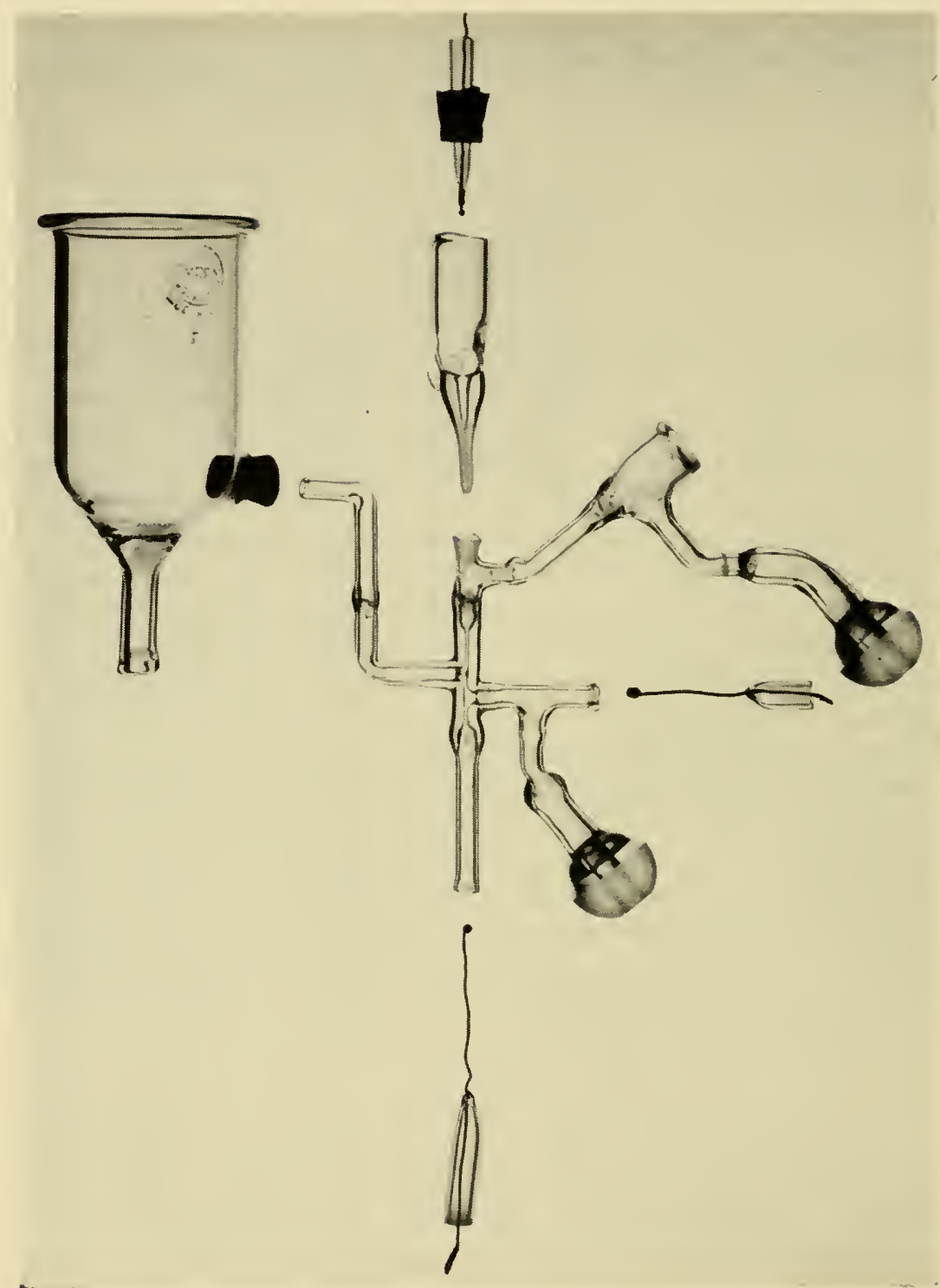

FIG. 3. Respirometer for measuring oxygen consumption of resting and active rat ganglia. Scale of photograph is indicated by the reservoir on the left, which is one inch in diameter. All glass parts are of Pyrex, except for the plugs in which platinum wires are sealed. Further description in text. 
1942 ) is used to measure the amount of oxygen remaining in a solution which has been pulled past the experimental tissue at a known rate. The essential glassware of such a respirometer, made to fit the superior cervical ganglion of an adult rat, is shown in Fig. 3.

During an experiment, the reservoir on the left was closed at the top by a stopper and was filled with a bathing solution continually equilibrated with $95 \% \mathrm{O}_{2}, 5 \% \mathrm{CO}_{2}$. The fluid was pulled through the respirometer by two motor-driven syringes connected to the ball and socket joints on the right. The ganglion was placed in the expanded upper portion of the vertical tube, with the postganglionic nerve pulled into the small channel in the ground glass plug at the top and the preganglionic nerve pulled down through the narrow channel below, so that its end lay in the lower expansion. Stimuli were applied between two platinum wires with tiny ball tips sealed through the glass plugs at the bottom and at the right. The preganglionic action potential could be recorded between a platinum electrode (not shown) which dipped into the reservoir on the left and a calomel cell (also not shown) which was connected to the bathing fluid beyond the upper ball joint. The postganglionic action potential was recorded between the same calomel cell and the platinum wire at the top. The electrode used for measuring oxygen concentration passed through a stopper inserted in the receptacle at the upper right, with the tip of the electrode lying in the small tube leading fluid away from the ganglion. Solution which passed the stimulating electrodes was carried away by the lower syringe, so that the oxygen measurements, which were made in the upper outflow, were not affected by events at the stimulating electrodes.

All the parts shown in Fig. 3, and also an assembly of stopcocks and flasks for changing solutions, were sterilized in an autoclave. In addition the bathing solutions were sterile and the ganglion was excised and inserted in the respirometer aseptically. These precautions were necessary because the ganglion was so small that under non-sterile conditions the empty chamber and the solutions flowing through it were sometimes found to consume about as much oxygen as the tissue itself. The whole apparatus was placed in a temperature-controlled box. An experiment lasting many hours, including changing of solutions, was performed without opening the box.

Fig. 4 shows a record of oxygen concentration in the solution leaving the ganglion. Oxygen concentration increases downwards in this record; the level for zero oxygen is considerably above the top of the figure. The oxygen scale has been thus inverted in order to make an upward deflection represent an increase in rate of consumption: obviously the faster the consumption, the less oxygen remained in the solution. The sudden descents shown in the record occurred when fluid was pulled past the tissue at an accelerated rate which was so fast that only a negligible amount of oxygen was removed from the solution. This was done for purposes of calibration and indicated the line of zero oxygen consumption. The rest of the scale, shown at the left of the illustration, could then be calculated, knowing the flow rate, the solubility of oxygen, and the weight of the tissue. At the arrow in this illustration the bathing fluid was exchanged for one containing sodium azide. After some brief and largely artifactual disturbances the rate of oxygen consumption fell, a well-known effect 


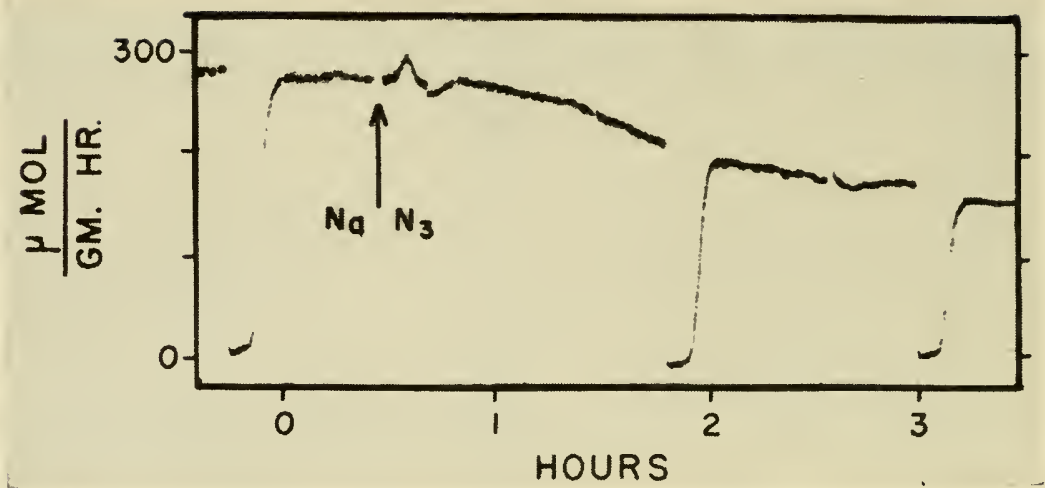

Fig. 4. Record of rate of oxygen consumption by a rat ganglion, obtained with the respirometer shown in Figure 3 . Sodium azide $(0.5 \mathrm{mM})$ was added at the arrow. Explanation in text.

of sufficient concentrations of azide. Two later calibrations show that the sensitivity of the system had not changed.

\section{Glucose and Lactate}

To measure exchanges of glucose and lactate, a ganglion was supported in the gas phase above a tiny glass vessel, so that it was exposed to moist $95 \%$ $\mathrm{O}_{2}, 5 \% \mathrm{CO}_{2}$ (Fig. 5). By raising the vessel, the ganglion was dipped into the solution for a fraction of each minute, in order to supply glucose and re-

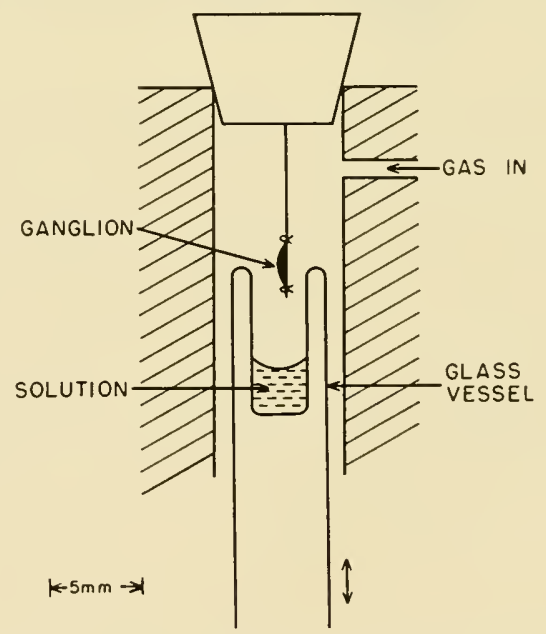

FiG. 5. Rat ganglion mounted for measurement of glucose uptake and lactate production. The glass vessel was raised to immerse the ganglion for about 15 seconds every minute. (Edwards and Larrabee, 1955). 
move lactate. The volume of fluid was kept small, 20 to 30 microliters, so that about $10 \%$ of the glucose was removed in one hour. Glucose was initially present in a concentration of $1 \mathrm{gm}$. per liter. At the end of about an hour the vessel was exchanged for one containing fresh solution. The accumulated samples were analyzed for glucose by the method of Park and Johnson (1949) and for lactate by the method of Barker and Summerson (1941). In these analyses the usual steps involving precipitation of proteins were omitted because there was no reason to expect much protein to appear in fluid bathing a ganglion with the connective tissue sheath still intact. The results on resting uptake of glucose did not differ significantly from those found earlier using a method based on glucose oxidase, which was more specific for glucose than that used here (Edwards and Larrabee, 1955).

\section{IV eights}

At the end of each experiment the tissue was dried at $90^{\circ}$ to $100^{\circ} \mathrm{C}$. for at least $1 / 2$ hour, which was sufficient to reach constant weight. All results are expressed in terms of dry weight of tissue, which was usually between 200 and 400 micrograms. The only exception is in Fig. 10, where rates are based on wet weights.

\section{Results}

\section{Resting Metabolism}

The average rate of resting oxygen uptake by 18 excised rat ganglia was $298 \pm 8$ micromoles per dry gm. hr. (mean \pm s.e.m.). This is not particularly interesting in itself. More information was gained by comparing this result with rates of glucose uptake and lactate production.

During the first hour or two after excision a typical ganglion produced a significant amount of lactate, about $1 / 3$ as many micrograms as glucose was consumed. Rates for both glucose and lactate rose slowly for several hours, but they kept in step, so that the differences between them stayed constant. The lactate presumably came from the glucose, since its production stopped when glucose was withdrawn. For further analysis we must consider possible destinies of glucose other than (a) lactate. Presumably some glucose was (b) oxidized to $\mathrm{CO}_{2}$ and water. Some may have been (c) polymerized to glycogen and some may have gone to (d) other miscellaneous fates, including loss as pyruvate, acetate, or other intermediates. Lacking definite information we will assume for the present that amounts going to glycogen and the "other" destinies were negligible. Our chief justification for this assumption is that, when oxidation was stopped by removal of oxygen, then all the glucose taken up (22.9 mg./dry gm. hr.) was recovered as lactate $(22.2 \mathrm{mg} . /$ dry gm. hr.), within the limits of accuracy of our methods. Thus the amount of glucose going to fates (c) and (d) must have been trivial, at least under conditions of anoxia. 
Proceeding on the assumption that all the glucose not converted to lactate was completely oxidized, some calculations can be made. Per gram of dry tissue per hour the average rates during the first two hours were $11.7 \mathrm{mg}$. of glucose consumed and $3.5 \mathrm{mg}$. of lactate produced. The difference, $8.2 \mathrm{mg}$. of glucose, was thus left over for oxidation. Assuming that this residue was all completely oxidized, stoichiometric relations permit the calculation that 273 micromoles of oxygen would have been required per dry gm. hour. Actually the observed oxygen uptake was 298 micromoles per dry gm. hour, as stated above.

Thus it is highly probable that exogenous glucose is the main substrate for resting oxidations in a ganglion, in agreement with conclusions for other nervous tissues. It is possible that under the conditions of our experiments there was, in addition, some oxidation of endogenous substrates, since the observed oxygen uptake exceeded the calculated rate by 25 micromoles per dry gm. hr. However, this difference is not statistically significant, since it is less than 2 times the standard error, as calculated from the standard errors of the glucose, lactate, and oxygen data. Moreover, there were some systematic differences between the conditions for the various measurements. We have thus been unable either to prove or disprove a slow oxidation of endogenous substrates under the conditions of our experiments. The important conclusion is that glucose is the major substrate for oxidation in a ganglion at rest.

Our results are in agreement with the conclusion reached by others that only a small fraction of the useful energy generated intracellularly is derived non-oxidatively from glycolysis. This is perhaps indicated most clearly by attempting to calculate the yield of so-called high energy phosphate bonds $(\sim P)$ from our metabolic measurements. Provisionally we have assumed that the consumption of each atom of oxygen was accompanied by the formation of three molecules of $\sim \mathrm{P}$, in agreement with average $\mathrm{P} / \mathrm{O}$ ratios observed under optimal conditions in various tissue preparations (Lehninger, 1953-54). The validity of this assumption will be considered below. We may also assume that in addition to the oxidative generation of $\sim \mathrm{P}$, two molecules of this material were formed for each molecule of glucose consumed, in accordance with the Embden-Meyerhof schema of glycolysis. Results of the calculations suggest that about $95 \%$ of the so-called high energy phosphate bonds were generated by oxidations (Table IV). This percentage must be regarded as only a rough approximation to the true value since it is by no means certain that the $\mathrm{P} / \mathrm{O}$ ratio is as high as 3 in intact cells (Lehninger, 1953-54). However, it is not unreasonable to assume that the normal $\mathrm{P} / \mathrm{O}$ ratio is at least high enough so that most of the useful energy obtained from glucose metabolism through mediation of phosphate bonds does indeed come from oxidations.

With the realization that the results can only be construed as approximations, we have also calculated the $\sim \mathrm{P}$ yields in the absence of glucose and in the ab- 
TABLE IV

Calculated formation of $\sim \mathrm{P}(\mathrm{mM} /$ dry g. hr.) in rat ganglia at rest, assuming $\mathrm{P} / \mathrm{O}=3$ and $\mathrm{P} / \mathrm{G}=2$

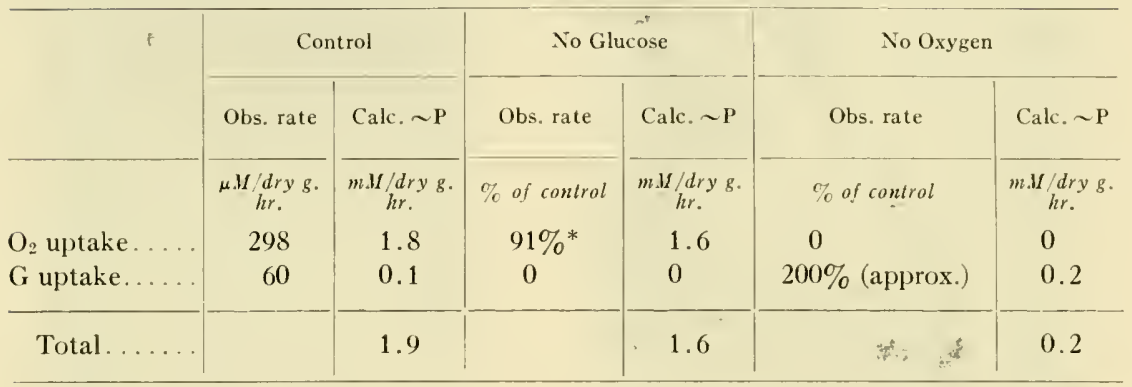

* Median of 8 experiments, 2 hours after withdrawal of glucose.

sence of oxygen (Table IV). These figures further suggest that the amount of non-oxidative energy yielded from glucose metabolism is normally a relatively small portion of the total energy supply.

\section{Metabolism During Activity}

Recently we have succeeded in measuring glucose and lactate exchanges during various states of activity in rat ganglia. This was made possible by the electrode assembly shown in Fig. 6. It was constructed of fine platinum wires imbedded in glass with projecting loops through which the nerves could be drawn. The ganglion and electrodes were dipped in the bathing solution once a minute. By coating the lower end of this structure with Desicote, good drainage was obtained when the solution was lowered. Thus stimulation was not short-circuited by fluid and action potentials could be recorded, usually free of stimulus artifact. There was relatively little decline in height of response during prolonged stimulation at frequencies up to 10 per second.

Using these methods, consistent and sizable increases in rate of glucose uptake were found during repetitive stimulation. The examples in Fig. 7 were caused by stimulation at 15 per second.

Large increments in rate of glucose uptake during stimulation were reported for excised spinal cords of frogs by Hirschberg and Winterstein (1917) and later for frog peripheral nerves by the same authors (1919). By contrast Holmes, Gerard, and Solomon (1930) were unable to find acceleration of carbohydrate disappearance during activity in nerve trunks from frogs or rabbits. In these latter experiments no exogenous glucose was supplied. Further results on frog nerve which might at first glance also seem to disagree with ours have been reported recently by Mullins (1953). Using tracer techniques, Mullins found no increase in yield of radioactive $\mathrm{CO}_{2}$ from tagged glucose during stimulation. But it turns out that our observations are reconcilable with those of Mullins, 

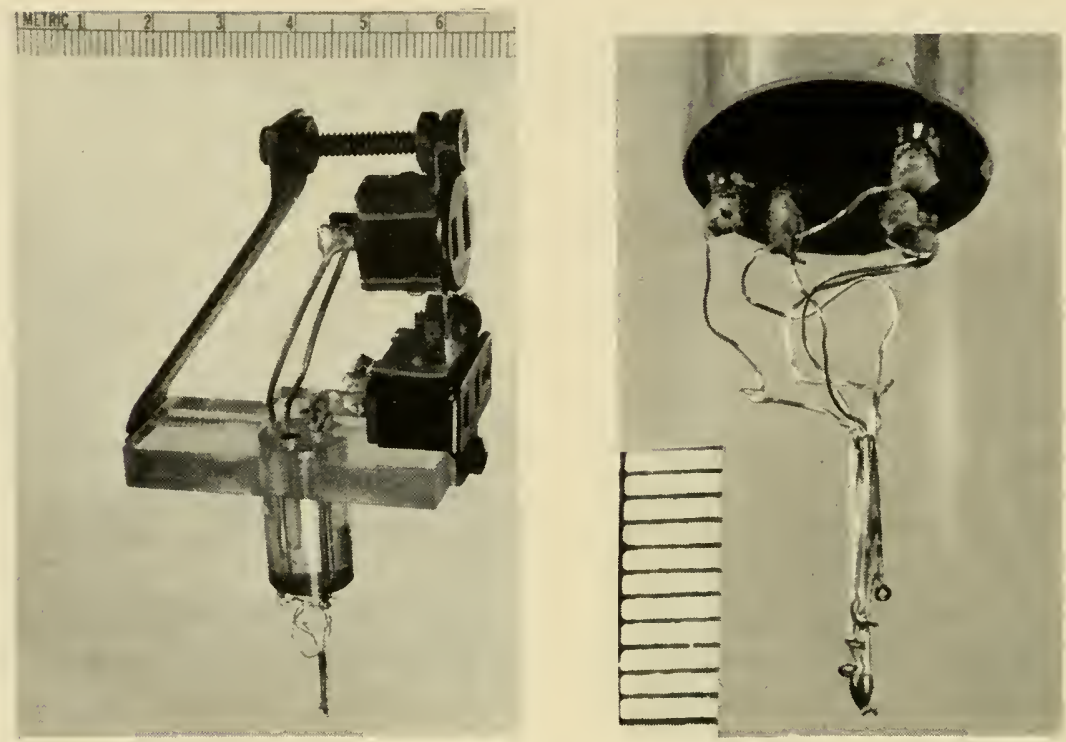

Fig. 6. Electrode assembly for stimulating and recording from excised rat ganglia while measuring glucose and lactate metabolism. This assembly fitted into the apparatus shown in Figure 5, in place of the stopper and wire. Left: The entire assembly. Scale at top is numbered in centimeters. Right: Enlarged view of the electrodes, imbedded in glass insulating sleeve. The two lower electrodes were for stimulation, the middle for ground connection, and the upper two for recording. Scale on the left in millimeters.
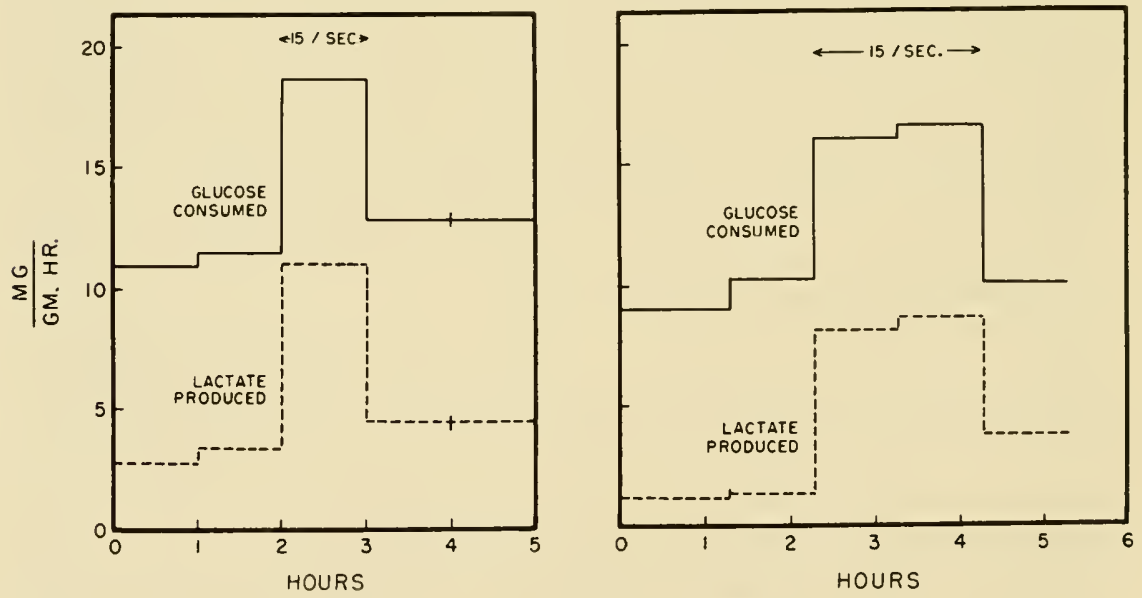

FIG. 7. Effects of activity on rates of glucose consumption and lactate production in two rat ganglia. Supramaximal stimulation was applied to the preganglionic nerve at a frequency of $15 / \mathrm{sec}$. luring periods indicaterl at the top. 
since in ganglia the extra glucose was apparently not oxidized, but was simply converted to lactate. In Fig. $\bar{\tau}$, for example, the increase in lactate production during activity was almost exactly equal to the increase in glucose uptake.

Certain control experiments may be briefly mentioned. The increments were shown not to be artifacts of the stimulating current, since their magnitudes were much smaller when the ganglion was cut off from the preganglionic nerve and the nerve was placed on the electrodes by itself. Therefore, the metabolic changes must have been associated with conduction and discharge of impulses. We have also shown that the acceleration of glycolysis is not due to a functional anoxia brought on by the increased rate of oxygen utilization. This was concluded from the fact that lowering the oxygen concentration in the equilibrating gas from $95 \%$ to $80 \%$ caused little or no alteration in the rates of glucose, lactate, or oxygen exchanges when the neurons were at rest. Even in the presence of $60 \%$ oxygen there were no consistent changes. It is possible that limitations of oxygen diffusion came into play at the highest rates of activity, and may then have influenced the metabolic responses. However, the phenomena with which we are concerned developed progressively, starting with the lowest frequencies of stimulation, where the oxygen supply was evidently adequate.

Glucose and lactate exchanges as a function of frequency of activity are shown in Fig. 8. Both rates increased with frequency. However, the difference between them remained approximately constant. This difference is a measure of the amount of glucose available for oxidation. Assuming complete oxidation of this amount, we can calculate the amount of oxygen required, with results shown as a function of frequency by the lower line in Fig. 9. The approximate constancy of the glucose available for oxidation is emphasized by the absence of upward or downward trend in this line. By contrast, the upper line in this figure shows the rate at which oxygen was actually consumed. This increased considerably with the frequency of activity. The difference be-

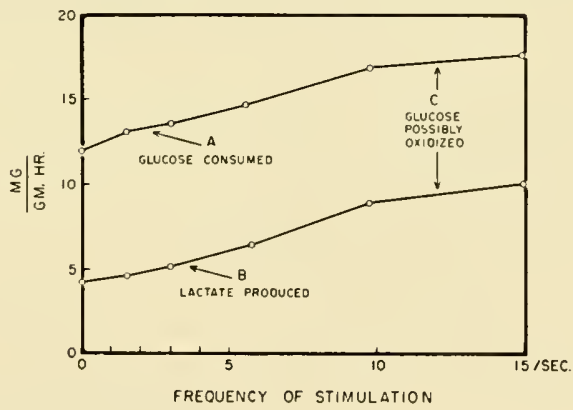

Fig. 8. Rates of glucose consumption and lactate production by rat ganglia as functions of frequency of supramaximal stimulation of the preganglionic nerve. Each point is the average of results on 2 to 5 ganglia. 


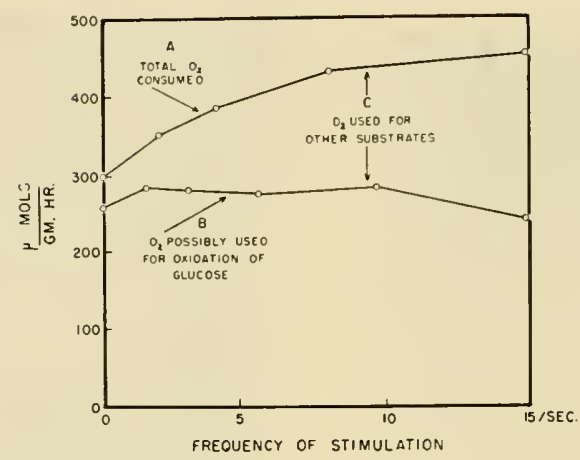

Fig. 9. Comparison of $(A)$ the rate at which oxygen was consumed by rat ganglia during supramaximal stimulation of the preganglionic nerve with (B), the rate at which oxygen would be required to oxidize the glucose which was consumed but was not converted to lactate.

tween these two curves must represent oxidation of substrates other than glucose - in other words endogenous substrates under the conditions of these experiments.

The conclusion of Holmes, Gerard and Solomon (1930) and of Mullins (1953), that the substrate for activity metabolism is not glucose, is thus supported and extended to an example of nervous tissue in another species; moreover, to a tissue which contains cell bodies and synapses as well as axons. In contrast with the older work we now find that glucose utilization is accelerated during activity. However, the final conclusion concerning the substrate for activity is the same, since this does not appear to be glucose. We may also recall recent evidence of Geiger, Magnes, and Dobkin (195t) that the extra glucose consumed in perfused cat brain during convulsions is all converted to lactate, just as in ganglia during activity.

Other examples of parallel changes in glucose uptake and lactate output. Conversion of the extra glucose consumed during activity, into lactate instead of into oxidation products, could conceivably be due to a rate-limiting step, operating at a maximal rate even under normal resting conditions, somewhere between pyruvate and the Krebs cycle. In this connection it is relevant to mention two other circumstances in which acceleration of glucose uptake was accompanied by an equal acceleration of lactate production.

One example was the spontaneous increase in rates during the course of an experiment, mentioned earlier in this paper. Thus, in the average of a number of experiments, glucose uptake increased about $30 \%$ in the first 5 hours after excision, while lactate output nearly doubled. However, the difference between the absolute rates, which represented the amount of glucose available for oxidation, showed no significant alteration.

The other circumstance in which acceleration of glucose uptake was matched 


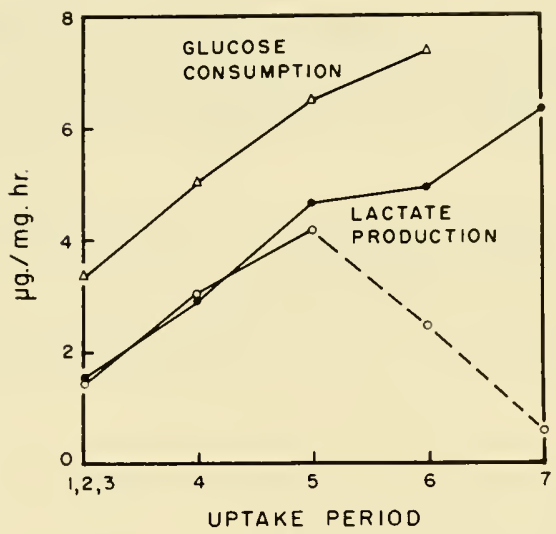

Fig. 10. Effect of pentobarbital $(0.5 \mathrm{mMI})$ on resting rates of glucose consumption and lactate production by rat ganglia. Glucose data are averages from five ganglia. Lactate production was measured on two other ganglia, each represented by a different symbol. Pentobarbital was added at the start of the fourth uptake period. The broken line indicates the effect of removing pentobarbital from one ganglion at the start of the sixth period. Each uptake period was about 50 minutes long. Rates in this figure are based on wet weight of tissue. (Edwards and Larrabee, 1955).

by acceleration of lactate production was caused by the application of pentobarbital in two exploratory experiments (Fig. 10). In each case, the output of lactate rose along a time course that closely paralleled that of the average glucose uptake of several other ganglia exposed to the same concentration (Edwards and Larrabee, 1955).

We thus conclude that there is some limitation in rate of conversion of glucose into materials other than lactate. It should be emphasized that under the conditions of our experiments this limitation was not due to limitation of oxygen diffusion, since the resting metabolic exchanges were not significantly affected by moderate reduction in external oxygen tension, as mentioned earlier.

\section{Significance of Acceleration of Glucose Metabolism During Actirity}

These observations raise a question as to the significance of the observed acceleration in rate of glucose uptake during activity. The extra glucose does not seem to supply additional substrate to the oxidative mill, since no more glucose appears to be made available for this use than during rest. Moreover, we can attach no special importance to the increase in the small amounts of energy yielded from reactions which convert glucose into pyruvate. This is because repetitive activity can still be elicited when lactate, which skips these steps, is substituted for glucose.

We are left with the possibility that the acceleration of glucose uptake may serve no useful purpose at all. Can it be just an irrelevant by-product of ac- 
tivity? Perhaps a useless consequence of increased intracellular inorganicphosphate or phosphate acceptors? Or an inadvertent result of increased intermingling of the ingredients of the hexokinase reaction, caused by transitory changes in the structure of the cell membrane where this reaction is supposed to take place?

Such a waste of material, although not categorically refutable, is at least teleologically unsatisfying. Moreover, there is evidence that the need for glucose actually does increase during activity, since failure of function in the absence of this material occurred sooner the more rapidly repeated was the nervous action (see below). This acceleration of failure was much greater than can be accounted for by accelerated depletion of endogenous substrate, at least on the basis of the few relevant data presently available. Consequently glucose seems to serve some need during activity, the nature of which is by no means obvious at the present time.

\section{Effects of Glucose Lack}

Failure of function. It has previously been reported that conduction of impulses along sympathetic nerve fibers and transmission through sympathetic ganglia is lost in the absence of glucose (Kahlson and MacIntosh, 1939; Larrabee and Bronk, 1952; Larrabee, Edwards and Ramos, 1951). For example, each time glucose was withdrawn in the experiment illustrated in Fig. 11, the response declined considerably after $10 \mathrm{~min}$. and was almost gone after 20 minutes. Each restoration of glucose was followed by recovery, but this was incomplete. Indeed, incomplete recovery, or almost none at all, was characteristic of the effects of temporary removal of glucose.

Rate of failure in the absence of glucose increased with the frequency of stimulation. This is in agreement with the fact that activity causes an increase in the normal rate of glucose uptake. With the ganglion kept at rest, or more precisely with stimulation once every few minutes to test capacity for response, failure was much slower than that illustrated: the postganglioic action potential of a rat ganglion then usually took $1 \frac{1}{2}$ to 2 hours to fall to half its initial height (Fig. 12, upper graph).

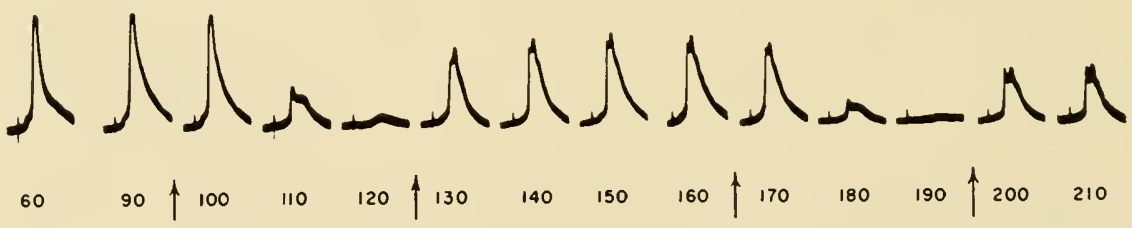

Fig. 11. Effects of glucose lack on the postganglionic response to stimulation of the preganglionic nerve of a superior cervical ganglion from a rat. Records were taken at 10 minute intervals during continual supramaximal stimulation at a frequency of 6 per second. Glucose was alternately withdrawn and restored at times indicated by the arrows. (Larrabee and Bronk, 1952). 


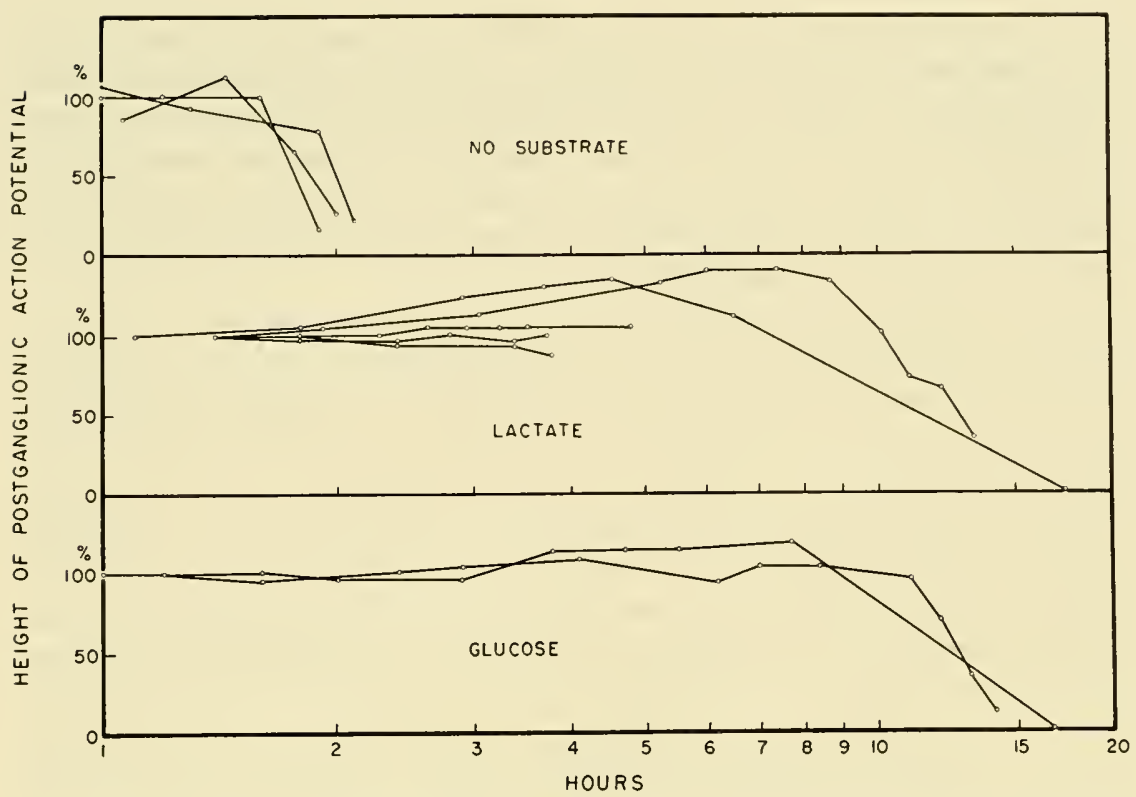

FIG. 12. Survival of postganglionic response in superior cervical ganglia excised from rats. Ganglia were placed in stirred solutions containing various substrates. The preganglionic nerve was stimulated supramaximally once every few minutes to test the capacity to respond. Time in hours after withdrawing glucose (upper graphs) or hours after excision (middle and lower graphs). Concentration of lactate, approximately $5.6 \mathrm{mM}$; of glucose, $5.5 \mathrm{mM}$.

Maintenance of oxygen consumption. Considering the evidence that glucose is the main substrate for resting metabolism, and the fact that capacity for function is lost when glucose is absent, it would be reasonable to expect that the rate of oxygen consumption should decline considerably following withdrawal of glucose. This is not at all what happens, at least for the first two hours after removal of glucose. During this period, in which the functional abilities of the neurons are drastically and largely irreversibly reduced or abolished, oxygen utilization is relatively unaltered. This observation, previously reported (Larrabee and Bronk, 1952), has recently been confirmed with improved techniques, including sterile conditions. Never did the rate of oxygen consumption fall more than $20 \%$ during the first two hours after withdrawal of glucose, and the change was usually much less than this. On the average oxygen uptake dropped only about $10 \%$. Oxygen consumption at a rate independent of glucose supply has been observed in the cerebral cortex of cats under conditions of perfusion by Grenell and Davies (1950).

\footnotetext{
${ }^{2}$ For additional details from more recent experiments concerning oxygen consumption, see Larrabee, Horowicz, Stekiel, and Dolivo, 1956.
} 
One possible interpretation of the smallness of the change in oxygen uptake after withdrawal of glucose, would be that glucose is not oxidized to a significant degree, even when available, but is all lost as lactate and other unoxidized materials. In this case the approximate agreement which we have found between the observed oxygen uptake and that calculated for glucose oxidation would be fortuitous, and the substrate for oxidation would be all endogenous, even when external glucose is supplied.

At the present time, however, we prefer to continue with the assumption, adopted elsewhere in this paper, that all the glucose which is not converted to lactate is normally oxidized. If this be true, our oxygen measurements show that the cells of a ganglion shift from glucose to the oxidation of endogenous substrates when the external sources of carbohydrate are withdrawn. It does not seem likely that glycogen serves as the substitute for glucose: if glycogen were oxidized at a rate sufficient to support the normal rate of oxygen uptake there is no obvious reason why function should not also be maintained. Possibly the endogenous substrates utilized during the glucoselack include proteins. In agreement with this suggestion are observations of others on brain tissue (Kimura and Ito, 1952) and measurements on ganglia in our own laboratory (Stekiel and Larrabee, 1955), which indicate that ammonia production increases when glucose is withdrawn.

Speculation on causes of failure of function. (a) Loss of energy production. Since oxygen consumption does not drop significantly when glucose is withdrawn, it is doubtful whether the decline in response should be ascribed to loss of energy production. In this connection it is relevant that oxygen uptake can be considerably depressed by other procedures without causing failure of function (Table $\mathrm{V}$ ).

The disappearance of the response during withdrawal of glucose could seemingly be explained on energetic grounds only by making the gratuitous (although possibly correct) assumption that the energy derived from oxidation of endogenous substrates is less efficiently coupled to the mechanisms for energy utilization than that derived from exogenous glucose.

TABLE V

Effects on oxygen consumption and postsynaptic response in rat ganglia

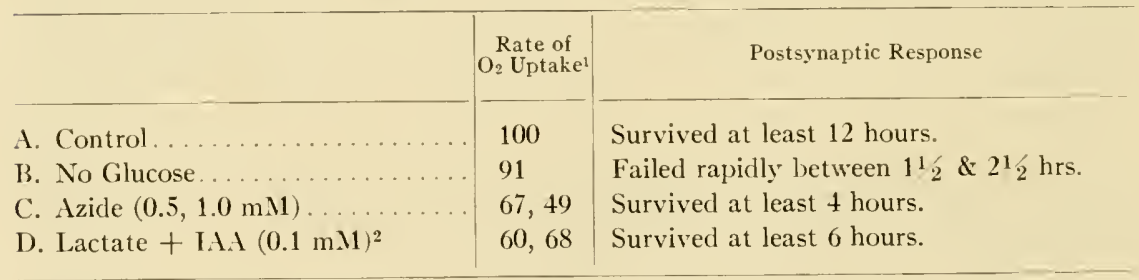

${ }_{1}^{1}$ Two hours after changing bathing solution, expressed as percent of rate at start.

${ }^{2}$ In the absence of glucose. 
In seeking other explanations for failure during glucose lack, we had at one time thought that there might be something especially important about the small amount of ATP formed non-oxidatively in glycolysis, perhaps because it was formed at some critical location in the cell. As it turns out, however, loss of this moiety cannot account for loss of function, since lactate, which does not generate the moiety, nevertheless supports function (Figure 12).

(b) Loss of acetylcholine generation. Another important role of glucose metabolism may be the generation of acetylcholine, as one of the products of acetyl Co-A. Therefore it is possible that loss of acetylcholine production is the critical defect in glucose-lack. The ability of exogenous lactate to support function can be explained on this basis, since lactate should be able to substitute for glucose in the manufacture of acetylcholine via familiar pathways leading through pyruvate and acetyl Co-A. Moreover Kahlson and MacIntosh (1939) found that liberation of acetylcholine during activity in a perfused sympathetic ganglion declined in the absence of glucose and could be restored, at least temporarily, by supplying glucose, lactate, or certain other substances. However, it is not established whether the acetylcholine deficit was actually the cause of failure, or merely one of several changes, some other of which may have been more intimately related to decline of neuronal function.

(c) Damage by oxidation of intracellular materials. We have also considered the possibility that the intracellular materials oxidized in the absence of glucose might include cellular constituents - possibly structural components - which are essential to conduction and transmission of nerve impulses and which are not readily replaced under our experimental conditions. This would explain the irreversibility of the failure of function. This irreversibility has puzzled us, for we have found no metabolic basis for it. Oxygen uptake, if it had been depressed at all by withdrawal of glucose, returned approximately to the control rate when glucose was restored, and so did the rates of glucose uptake and lactate production. Nevertheless, the restoration of these several rates to their normal level failed to bring back the capacity for either nerve conduction or symaptic transmission, even after several hours. From this point of view the essential role of glucose in the economy of the cell may be to supply a substrate which satisfies energetic requirements without oxidation of intracellular materials. Perhaps oxidation of the latter provides considerable energy, and may be useful during hypoglycemic emergencies of brief duration, but ultimately results in irreversible damage if glucose is long withheld. ${ }^{3}$

(d) Damage by products of endogenous substrates. In concluding these specu-

${ }^{3}$ In oral presentation at the Symposium it was suggested that certain enzymes, normally occupied with glucose metabolism, might turn to other substrates in the absence of glucose. These enzymes were likened to mischievous children-when not given something suitable to do, they proceed to take the house to pieces. This frivolous analogy is mentioned here to explain any references to the "mischievous enzyme theory" which may survive revision of comments by others in the ensuing discussion. 
lations, it may be suggested that metabolism of endogenous substrates during lack of glucose might produce irreversible changes, not only directly by removing essential materials as proposed above, but also secondarily by the formation of toxic products. Such products might include ammonia, for example, or the increased intracellular $\mathrm{pH}$ resulting both from production of ammonia and from decreased formation of lactic acid.

\section{References}

Barker, S. B. and W. H. Summerson. 1941. The colorimetric determination of lactic acid in biological material. J. Biol. Chem. 138: 535-554.

Brink, F., Jr., D. WV. Bronk, F. D. Carlson, and C. M. Connelly. 1952. The oxygen uptake of active axons. Cold Spring Harbor Symp. Quant. Biol. 17: 53-67.

Butler, T. C. 1950. Theories of general anesthesia. Pharmacol. Rev. 2: 121-160.

Carlson, F. D., F. Brink Jr., and D. W. Bronk. 1950. A continuous flow respirometer utilizing the oxygen cathode. Rev. Sci. Inst. 21: 923-932.

Davies, P. WT. and F. Brink Jr. 1942. Microelectrodes for measuring local oxygen tension in animal tissues. Rev. Sci. Inst. 13: 524-533.

Doty, R. W. and R. W. Gerard. 1950. Nerve conduction without increased oxygen consumption: Action of azide and fluoroacetate. Am. J. Physiol. 162: 458-468.

Edwards, C. and M. G. Larrabee. 1955. Effects of anaesthetics on metabolism and on transmission in sympathetic ganglia of rats: measurement of glucose in microgram quantities using glucose oxidase. J. Physiol. 130: 456-466.

Forbes, A., A. F. Battista, P. O. Chatfield, and J. P. Garcia. 1949. Refractory phase in cerebral mechanisms. Electroencephalography and Clin. Neurophysiol. 1: $141-175$.

Geiger, A., J. Magnes, and J. Dobkin. 1954. Non-carbohydrate sources of excess energy utilized by the brain during convulsions. Fed. Proc. 13: 52-53 (Abs.).

Greig, Margaret E. 1947. The effect of ascorbic acid in reducing the inhibition of brain metabolism produced by pentobarbital in vitro. J. Pharmacol. Exptl. Therap. 91: $317-323$.

Grenell, R. G. and P. W. Davies. 1950. Respiration of cerebral cortex in vivo, in the absence of glucose. Fed. Proc. 9: 52 (Abs.).

Hirschberg, E. and H. Winterstein. 1917. Metabolism of sugar in the central nervous system. Z. f. Physiol. Chem. 100: 185-202.

Hirschberg, E. and H. Winterstein. 1919. Metabolism of the peripheral nervous system. Z. f. Physiol. Chem. 108: 27-37.

Holmes, E. G., R. W. Gerard, and E. I. Solomon. 1930. Studies on nerve metabolism. VI. The carbohydrate metabolism of active nerve. Am. J. Physiol. 93: 342-352.

Jarcho, L. W. 1949. Excitability of cortical afferent systems during barbiturate anesthesia. J. Neurophysiol. 12: 447-457.

Kahlson, G. and F. C. MacIntosh. 1939. Acetylcholine synthesis in sympathetic ganglion. J. Physiol. 96: 277-292.

Kimura, Y. and K. Ito. 1952. Correlation between metabolisms of carbohydrate and nitrogen in brain tissue. Sci. Papers of the College of Gen. Ed., Univ. of Tokyo, Biol. Part 2: 157-173.

Larrabee, M. G. and D. IV. Bronk. 1952. Metabolic requirements of sympathetic neurons. Cold Spring Harbor Symp. Quant. Biol. 17: 245-266.

Larrabee, M. G., C. Edwards, and J. G. Ramos. 1951. Glucose requirements of sympathetic ganglia. Fed Proc. 10: 79 (Abs.). 
Larrabee, M. G. and D. A. Holaday. 1952. Depression of transmission through sympathetic ganglia during general anesthesia. J. Pharmacol. Exptl. Therap. 105: $400-408$.

Larrabee, M. G., P. Horowicz, W. Stekiel, and M. Dolivo. Metabolism in relation to function in mammalian sympathetic ganglia. In: The Metabolism of the Nervous System. D. Richter, ed. Proceedings of the Second International Neurochemical Symposium. London. Pergamon Press. 1956, in press.

Larrabee, M. G. and J. M. Posternak. 1952. Selective action of anesthetics on synapses and axons in mammalian sympathetic ganglia. J. Neurophysiol. 15: 91-11t.

Larrabee, M. G., J. G. Ramos, and E. Bülbring. 1952. Effects of anesthetics on oxygen consumption and on synaptic transmission in sympathetic ganglia. J. Cell. \& Comp. Physiol. to: 461-494.

Lehninger, A. L. 1954. Oxidative Phosphorylation. The Harvey Lectures, Series 49: 176-215. (1953-5t).

Lewis, J. L. and H. McIlwain. 1954. Action of some ergot derivatives, mescaline, and and Dibenamine on the metabolism of separated mammalian cerebral tissues. Biochem. J. 57: 680-684.

McIlwain, H. 1953. The effect of depressants on the metabolism of stimulated cerebral tissues. Biochem. J. 53: 403-412.

Marshall, W. H. 1941. Observations on subcortical somatic sensory mechanisms of cats under Nembutal anesthesia. J. Neurophysiol. t: 25-43.

Marshall, W. H., C. N. Woolsey, and P. Bard. 1941. Observations on cortical somatic sensory mechanisms of cat and monkey. J. Neurophysiol. t: 1-24.

Mullins, L. J. 1953. Substrate utilization by stimulated nerve. Am. J. Physiol. 175: $358-362$.

Park, J. T. and M. J. Johnson. 1949. A submicrodetermination of glucose. J. Biol. Chem. 181: 149-151.

Quastel, J. H. 1952. Effects of drugs on metabolism and physiological activity of brain. In: The Biology of Mental Health and Disease. A Symposium. The 27 th Annual Conference of the Milbank Memorial Fund. New York. Paul B. Hoeber.

Rosenberg, A., L. Buchel, N. Etting and J. Levy. 1950. Action des narcotiques sur le metabolisme du cerveau. Compte Rendus des Seances de l'Academie des Sciences 230: $480-482$.

Stannard, J. N. 1939. Separation of resting and activity oxygen consumptions of frog muscle by means of sodium azide. Am. J. Physiol. 126: 196-213.

Stekiel, W. and M. G. Larrabee. 1955. Unpublished.

Webb, J. L. and K. A. C. Elliott. 1951. Effects of narcotics and convulsants on tissue glycolysis and respiration. J. Pharmacol. Exptl. Therap. 103: 24-34.

Chatruan Gerard: Thank you very much. I cannot resist pointing out that maybe the thing that worries you most of all in this is itself going to go up the chimney somehow because, as you know, it is possible for brain to continue functioning for several hours with no glucose at all. I can also not resist pointing out that the mischievous enzyme theory comes back to the same problem that was raised with oxygen lack in nervous system and the fact that there is a survival time, a revival time and a recovery time which are closely related. And 
the recovery time varies with the metabolic rate and with the survival time, all of which would fit in with that same general notion.

\section{Discussion}

Led by R. G. GRENELL

One of the things that I am rather impressed with about these papers is that they have strengthened a belief of my own-and one held by many others - that the more experiments we do the worse our confusion. Usually most of what we have believed turns out not to be so. This has been a problem of mine for some time. I think it started with the experiments that Dr. Davies and I did some 5-6 years ago (reported in Federation Proceedings), in which we discovered that our faith in glucose had been misplaced, and that the brain, at least for certain phases of its work, could get along without any exogenous glucose for several hours. This disturbing observation was followed shortly by another; namely, that narcotics in just enough concentration to anesthetize (i.e., low concentration) do not significantly decrease the rate of oxygen consumption. At least this particular part of the oxidative machine was not what it had been thought to be. Before long it got so that we weren't sure we could find anything that was what it used to be.

In more recent days we have come to believe that uncoupling, too, may not be all we may have been led to believe.

These observations leave us in a state of extreme anxiety, and we have begun to look for some sort of correlation we could make that might explain a number of the factors being talked about this afternoon. One of the first questions of interest here is that of the differential biochemistry of cells of the central nervous system. Can we relate chemical structures to integrated activity? Further, can we localize the structures which are integral to these processes? When we talk about molecular structure, is there any identification of the structure within the cell, in the membranes, or outside the cell? Such questions lead to attempts to separate chemical effects in these areas and their relationship to activity. Part of the attack in this vein is demonstrated by the difference in approach of Dr. Fischer's work and that of Dr. Larrabee. Another type of approach is one in which we have used studies of narcotic action to give us a certain kind of information concerning the common mechanisms by which neurons react.

The question of common reactive or adaptive mechanisms is one of great interest. Some years ago, Dr. Ebbe Hoff and I spent some time reviewing the literature relative to structural alterations in the central nervous system in a series of conditions including anoxia, anemia, carbon monoxide poisoning, anesthesia poisoning, hypoglycemia, vitamin deficiency, etc. We were able to observe that, in general, such stresses evoked similar lesions in similar areas of the brain. There appear to be certain inherent properties of re- 
sistance or susceptibility in various cells or cell groups-perhaps one should say metabolic differences from group to group-but the overall picture is fairly constant. The structural similarity can also be shown to be associated with functional likeness. No one, for example, would deny certain similarities in behavior of a person suffering from oxygen want to one under the influence of alcohol or narcotics (at certain stages). In a narrower sense, we appear to be dealing with various phases of a cell problem which has become the more or less specialized domain of the neuron-i.e., excitation and depression, and the ancillary metabolic factors. Concerning the latter, let us look for a moment at the problem of the physico-chemical factors responsible for drug "addiction", as well as for the production of tolerance in habituated individuals and withdrawal symptoms when access to the drug is terminated. It is not impossible to theorize that all of these situations are directly related to initial, reversible effects of the drug on the molecular orientation of specific cell membranes, associated with secondary effects on ion shifts and other intracellular equilibria. Setting aside, for the moment, the "psychological" factors involved in "addiction", and considering this problem from a purely mechanical point of view-it could be suggested that following the chronic intrusion of narcotic molecules into the neuronal membranes, certain intracellular chemical changes occur which relate to "adaptation" or "tolerance." At the same time the structure of the cell (as well, perhaps, as its biosynthetic mechanisms) and the membranes is altered. If, subsequent to these changes in structure the drug is withdrawn, it would not be at all surprising to expect a violent reaction leading to destruction of new equilibria and instability of excitable structures. Addiction could be looked upon as a need for alcohol or other narcotics to maintain the new steady state level of the cell. Withdrawal symptoms might then be the acute result of loss of the new steady state. Experiments are in progress to check some of the steps in this theory.

It has been apparent that neither decreased absorption nor increased oxidation of alcohol seem to be the answer to the production of tolerance to it. The altered response of the individual can be explained at this time only by the suggestion of physico-chemical changes in certain neurons, associated with their excitability cycle. This alcohol problem is closely related to that of narcosis; indeed, the analogy between the effects of alcohol and of certain narcotics is remarkable. Several fundamental considerations arise from such relationships:

(1) Such similarities of structure and function must reflect the involvement of common biochemical and/or physico-chemical elements; and,

(2) These common mechanisms may be divided, for purposes of investigation, into two sets, partially related to each other and termed intraneuronal and membranal (it must be understood that this term refers not only to the 
'membrane' around the cell, but to other membranes as well, e.g., mitochondrial membrane and nuclear membrane).

The relationship between these two sets of mechanisms is brought out clearly, if, for example, one considers that the initiation and conduction of nerve impulses are manifestations of electrochemical processes localized in the surface structure of nerve cells and their axons. Action currents develop subsequent to changes in structural orientation at the surface. Brink has pointed out that such processes are "self-limiting and cyclic, being completed by cellular processes that restore the original excitable state." The molecular arrangements in the resting cell that are "capable of such a transition from one state to another" have been referred to as a metastable structure (Brink) in contrast to the excitable structure. The metastable structure responds to certain stimuli and controls ionic fluxes which give rise to potential changes. The stability of the membrane would depend on the molecular organization of this structure, and consequently, one would suppose that the initial, fundamental effects of alcohols could be at this locus.

Alcohols in the proper concentrations can be considered as narcotics or anesthetics. Evidence exists (Arvanitake; Graham; Hensel and Zotterman) that narcotics in concentrations lower than that necessary to induce narcosis act to produce stimulation of neural structures. Mullins has suggested that if a chemical agent lowers the threshold to the point of self excitation before causing narcosis, similar self excitation of olfactory receptors can occur when a certain mole fraction of chemical agent has been reached in their membranes. On the basis of a recent series of experiments we wish to suggest that ethyl alcohol acts in this way to produce stimulation in low concentration and depression in high concentration.

Using the local perfusion technique, ethyl alcohol in concentrations from 5 $\mathrm{mM}$. up to $800 \mathrm{mM}$. was perfused through a pial vessel directly into a localized area of the cerebral cortex of the cat. Evoked responses were obtained from the dendrites and cell bodies in the perfused area by direct electrical stimulation (bipolar stimulating electrodes connected to a Grass stimulator). Experiments thus far indicate that stimulation is obtained with concentrations between 5 and $25 \mathrm{mM}$. Concentrations of $50 \mathrm{mM}$. and above induce depression. During the perfusion of $5 \mathrm{mM}$. ethanol stimulation-i.e., increase in amplitude, for example, of the dendritic response has been seen, of as much as $65 \%$. Thus far some variation in the degree of stimulation or depression by a particular concentration has been observed from animal to animal. It is quite probable that this is real-and not error-and related to other factors which have affected the state of the membranes and thus of the excitability of these cells at the particular time during which the perfusion took place.

It must be noted that depression of the response can be induced by concentrations far below those which significantly inhibit the rate of oxygen consump- 
tion in vitro. Determinations have been carried out of the rates of oxygen consumption of homogenates, exposed in the Warburg apparatus, to increasing concentrations of methyl, ethyl, n-propyl and n-butyl alcohols. There is no question but that high enough concentrations can severely depress oxygen consumption. It is also apparent that these concentrations are much higher than those necessary to alter excitability, as well as those in the blood of individuals who are unquestionably "under the influence" of alcohol. Again the findings would indicate an initial membrane effect (reflected, presumably, as changes in polarization) not involved with oxidative mechanisms.

These effects of alcohol on activity and oxidative mechanisms appear to be very similar to those of a large group of sedative and hypnotic drugs (narcotics). Narcotics too, have been shown to stimulate before they depress. Moreover, common physico-chemical mechanisms must be responsible for the induction of narcosis (as well as for recovery from it), inasmuch as so many structurally different compounds are effective (alcohols, barbiturates, urethanes, etc.).

Many factors possibly associated with narcosis have been suggested as being the foci of these common mechanisms, among them the relative solubilities of various compounds in the lipoid and protein phases of the cell membrane, interference with respiratory enzymes, and blocking of oxidative breakdown and high energy phosphate bond production in the cell. Much work has been concerned primarily with the biochemical reactions in the cell which revolve around the utilization and synthesis of adenosinetriphosphate (ATP). It has been suggested that narcotics interfere with the direct acetylation process. Recent studies show that this is not the case (alcohols included). Some investigations appeared to indicate that the answer lay in direct suppression of the oxidative synthesis of ATP or in uncoupling of phosphorylation from oxidation. That synthesis is not interfered with is shown by recent results in our laboratories; that uncoupling is not the answer is indicated by the fact that dinitrophenol, gramicidin and aureomycin, which are among the most effective uncoupling agents, are not known to have any significant narcotic or anesthetic properties.

Many investigators have suggested effects on brain respiration. However, generalized inhibition of respiration is not what one should look for. Narcotics and anesthetics-and alcohols - in sufficient concentration significantly inhibit oxygen consumption of cerebral tissue. However, several points are of importance here. The first is the problem of concentration of the narcotic. Many of these substances in physiological concentration do not inhibit respiration-in fact, low enough concentrations may even stimulate. Concentration is not the basic factor, but it becomes necessary to refer to the thermodynamic activities.

A second point involved here is that the rate of oxygen consumption is not the primary factor to be dealt with. The oxidative processes may be involved without being reflected by a change in the rate of oxygen consumption. There 
may, on the contrary, be shifts in high energy phosphate synthesis in anaerobiosis, group transfer and uncoupling. It is extremely unlikely from both the biological and chemical points of view that all narcotic substances, e.g., act on one specific enzyme path-even if their action is primarily on intracellular enzyme systems. It is well to remember that there are several enzymatic processes involved in the action of hypnotics, sedatives and narcotics:

(1) Glucose to pyruvic acid (glycolytic process).

(2) Pyruvic acid oxidized to carbon dioxide and water in the cyclophorasemitochondrial system.

(3) Process by which acetylcholine is generated and triggers the impulse (including the process of conversion of bound to free acetylcholine) The utilization and/or generation of energy by the system is of the first importance. Our results with narcotics have shown that in no case does the net synthesis of ATP appear to be significantly depressed by the narcotic substances in reasonable concentration. The question still remains, in riro, of possible effects on ATP utilization. Our recent results have led us to believe that there is a direct relationship between ATP utilization and the activity level of the nerve cells. In both rat and cat, ATP in the cortex increased markedly with anesthesia. There is an increase following administration of chlorpromazine $(10 \mathrm{mg}$. $/ \mathrm{Kg})$ the amount of this increase varies in different areas and is most marked in the thalamohypothalamic area. Stimulation, on the other hand, (either in the form of direct electrical cortical stimulation or consequent to injection of metrazol) produces a very large drop in the ATP level.

This correlation of activity level and ATP concentration (which appears to be related to the effects, for example, of certain so-called tranquilizing drugs) suggests a partial explanation of some of the problems discussed this afternoon. Perhaps there is a clue here to mechanisms of transport across the cell membrane and their relation to molecular factors which enter into basic cell activity.

Dr. McElroy: Dr. Larrabee raised several questions which were very interesting; one, I think needs a little more clarification. That is the question on what one means by different metabolic pathways, whether one means a source of substrate, different types of substrate, or whether one means an entirely independent metabolic mechanism other than those we already know.

Another point is on the calculation of the ATP. I do not see how one can make an argument that the energy level is not important on the basis of those calculations because until you know the mechanism and extent of the electron transfer through the oxidative pathway one cannot decide whether you should use the $\mathrm{P} / \mathrm{O}$ ratio of three for these calculations. In other words, it could be, if you want to, an alternate pathway, and this could be a non-phosphorylating pathway for electron transport. One can make a calculation and say that the ATP should be high, but it does not necessarily mean that it is high.

The other thing is on the withdrawal of glucose. If you remove glucose, this 
is going to free your pathway for the breakdown of amino acids and fats and possibly other reserves. I think that one needs to consider these several possibilities in trying to eliminate metabolic activity as a supporting mechanism for electrical impulses. And in the case of the nerves, it seems to me perfectly possible, if you want to consider ATP as the energy reservoir, that one could make a point of having a high reservoir in the nerve which can be pulled slowly for activity. One may then get a stimulation of oxygen uptake because of the removal of this ATP. One still would have enough reserve to keep activity going for a long time in the absence of glucose. So I do not see that there is any inconsistency in the idea that the ATP level might be important in supporting nerve functions.

Dr. LARRABEE: I do not believe there is any special answer. I think Dr. McElroy has upheld me in emphasizing the speculative and hypothetical nature of the way we are thinking.

Dr. MCELroy: The direct question is a case of whether you are inhibiting metabolic activity when you inhibit nerve impulses. I did not see how you could come to that conclusion.

Dr. LARRABEE: All I can say is that one cannot tell whether the effect is primarily on an enzyme or not.

Chatruan Gerard: It seems to me the issue is very clear, as long as metabolism goes down directly proportional to the physiological response presumably it is because units are falling out.

Dr. LARRABEE: I think that McIlwain's proposal on this is probably in point. What he said is essentially that as soon as we learn how various aspects of metabolism change with activity then we can examine how these various aspects change when we apply inhibitors. If the inhibitor is acting non-metabolically then we can expect all aspects of metabolism to change in the same way as if excitation were withdrawn. A metabolic inhibitor, on the other hand, should cause a predominant effect on some particular portion of the metabolic system.

DR. ABOoD: We have been working with Dr. Geiger on his perfused cat brain preparation and during glucose perfusion the cat is able to go on functioning, that is, with normal reflexes. We have been able to show that, during this time, some things are disappearing in the brain. For example, we can show that the microsomes in the region of the cortex vanish almost completely in some areas. As you know microsomes are rich in lipids and proteins and on the basis of what we find in the plug we cannot account for all that disappears from the brain. So, apparently, some non-carbohydrate substance is being utilized.

Dr. Larrabee mentioned that during glucose-free perfusion of these preparations, activity gradually disappeared. We have noticed the same thing. We have also shown that when the microsome fraction in certain areas of the cortex goes down to $50 \%$ of the normal, electrical activity in the 
brain begins to disappear. So apparently there is something that is different about this glucose perfusion. Incidentally, this same type of thing seems to happen during stimulation of the brain-the metabolism shifts over to a noncarbohydrate type. Geiger, I think, has found this quite definitely and here, again, the effect is quite apparently an irreversible one. There is something being irreversibly destroyed in the brain which is part of the structural integrity of the brain.

In connection with this problem of the function of ATP, there are certainly many difficulties. First, I am curious to know what happens if ATP is going uP during anesthesia, or after chlorpromazine? What happens to the phosphocreatine? After all, there is some kind of equilibrium between the phosphocreatine and the ATP. If the phosphocreatine were to go up and the ATP to go down it would be another one of these anomalies. Also, it has been suggested that during increased electrical activity, in the brain, there is a decrease of phosphorylization. Now, it is very possible, as Dr. McElroy pointed out, that there may be some sort of non-phosphorylitic conveyance of electrons, but so far there is no evidence that such a thing happens in the brain.

Dr. Grenell: I certainly agree with what Dr. Abood says about most of this, but infortunately, I do not know what happens to the phosphocreatine. We are hoping to find out.

But there is an interesting point that I would like to add. We recently have started to look into what happens with insulin. This is a very confused business, of course, but we did notice that when a cat was injected with enough insulin to put a human being into deep insulin shock, the response to stimulation (direct electrical stimulation on the cortex) increased. Now, perhaps the old story of the EEG flattening out may be true, but the cortical response to an adequate stimulus is another matter. I think if we take our results and compare them with the results that Dr. Magoun obtained not long ago with auditory click responses that they are quite similar. His cortical auditory click response went up in the presence of insulin. Perhaps what one has to think about in the absence of glucose, no matter how you produce it, is that the adequate stimulus for the new threshold is different. The ordinary mechanisms that are producing the spontaneous activity are now shifted over to something else. If we do look to other substrates in the way of fat or protein or what you will, we may find some other story going on here. It is an entirely different story. Actually, if you do shift to an adequate stimulus for the new substrate then your activity picture becomes something else. I am not sure how the ATP values are going to turn out.

Dr. Jolin Nurnberger (Institute of Living, Hartford, Conn.): I wanted to add just a little to what Dr. Abood has said and to what Dr. Larrabee mentioned, that is, the possibility that the brain can use a variety of metabolizing agents in addition to glucose. Some of our studies indicate that within $15 \mathrm{~min}$ - 
utes after exposure in the rat there is a $12-15 \%$ decrease in the total nitrogen. This is not a concentration change, but an actual change of amount and very rapid in the presence of adequate nutrition. Also, I was a little taken aback when Dr. Larrabee mentioned the fact that unruly enzyme systems might have to deal with other than glucose metabolizers such as glutamine, since decarboxylase, deaminase and transaminase have been demonstrated, sometimes in unusually high concentration, in the neural tissue, to channel substances other than glucose into the tricarboxylic acid cycle to support oxidation, for example.

I noticed that Dr. Grenell and others referred to grams wet weight or dry weight in terms of their enzyme and other studies. There is a possibility that since we are talking about cellular metabolism perhaps we should refer to the actual cell density of the tissues we are studying. With Dr. Gordon, I have developed a very simple little technique for actually enumerating the number of neuron and non-neuron nuclei in various parts of the brain and I would like to give you some comparable data which we have on a striate cortex. There are a variety of forms. In the macaque and in two humans (a $16 \mathrm{yr}$. old and a $77 \mathrm{yr}$. old), if we take the total nuclei per gram of wet tissue, which is the usual reference (total nuclei, by actual count, is the neuronal nuclei plus the glia nuclei) we find 10.3 for the macaque, 9.45 for the $16 \mathrm{yr}$. old woman and 9.10 for the $77 \mathrm{yr}$. old man. The neuronal nuclei per gram of wet striate cortex were found to be 1.40 for the rat, 1.42 for the macaque, 1.75 for the young woman and 1.47 for the older man. I think that possibly the cellular references which can be obtained with extraordinary ease might be able to reveal some significant variations in metabolic activity whereas a nitrogen reference might not. For glia and endothelial nuclei, it is 88.7, 77.7 and 5.63. These are all nuclei times $10^{7}$ per gram wet striate cortex. One of the very surprising things is the relative uniformity of the density of cells, neurons and glia per gram wet striate cortex in, I would say, widely variant forms.

DR. LARRABEE. 'These mishievous chaps have certainly grown since I tossed them out! I do think that one thing to be remembered is that, whatever happens in the cell in the absence of glucose, it camnot be ascribed to destruction of one of the enzymes involved in glucose metabolism, for example, because glucose uptake, lactate production, and oxygen consumption all return to the original rates when glucose is restored. The capacity for impulse conduction and synaptic transmission, nevertheless, is not restored.

Dr. Folch-Pr: I don't think we have mentioned that glucose is not what it used to be and certainly biologists are not what they used to be. Because here we are forgetting one important fact and that is the oxygen. McIlwain cuts a slice of cortex, puts it in the icebox, comes the next day and he still gets good oxygen potential, which does not mean he gets activity for hours. He doesn't claim that. I would like somebody to comment on when we stop dealing with a live brain (in the same sense as a live muscle) and when do we just have the 
potential of the biochemical components of a dead tissue, a tissue that cannot come back?

Dr. Louis Sokoloff (National Institutes of Health): I wondered whether the EEG picture or the behavior pattern of a comatose human being, reflects the normal brain? Does the presence of electrical activity mean anything?

DR. LARRABEE: I would like to ask Dr. Abood how long these brains were perfused without glucose? Also, whether this is an ordinary perfusion or a recirculation system?

DR. ABOOD: The brain will survive about two hours and there is a gradual diminution of the electrical activity. It is not perfectly normal during that time and it is a recirculated perfusion.

Chatruan Gerard: Let me add a word here for some clarification. There have been biopsies made on the brain throughout and there is no change in the carbohydrate reserve in the brain. There is no glucose in the fluid going to the brain and there is no loss in the carbohydrate in the brain and it functions not only as tested by the electrical manifestations but as tested also by the reflex activity, which partly meets the point there. The question that Folch-Pi throws out, I think, is a searching and important one. What some of these lines of discussion come down to, it seems to me, is best exemplified in these experiments on the phosphorylating capacities of the isolated brain mitochondria that we did a couple of years ago. I will merely remind you this was precipitated by McIlwain's visit to our laboratory when he tried and failed to get any electrical evidence of impedance change in brain slices even though they were giving the biochemical responses to shocks applied to them.

In the absence of that, our conclusion was that these were not physiological responses and we went off in another direction. We found that isolated brain mitochondria, which we never for a moment thought were giving physiological responses, give exactly the same biochemical changes on being exposed to shocks in the suspension medium. The significant thing about the changes, as finally worked out with the aid of tracer phosphate, was that the decrease in phosphate associated with the electrical pulses, what you might call excitation, was primarily a decreased formation rather than an increased breakdown, which upsets the whole energy aspect and throws it into some relationship to excitability.

But, if there is a basic tie-up between excitation and metabolic change that is essential to or meaningful for the functioning of cells-one would hate to not think so-then certainly, important fragments of that remain intact when you have gotten inside the cell and you have no cell membrane and you are just down to the particulars.

Is that at all responsive to your question?

1)R. Folch-P1: Yes, I think that is the way to look at it.

DR. MARSHALL: I would like to ask for a little more rigorous clarification of the definition or specification of what people mean by electrical stimulation 
and electrical activity. I think that is where a lot of these misunderstandings arise and in these days the literature is always overloaded and nobody can write a paper that is long enough to describe this work adequately.

If I understood Dr. Grenell's remarks correctly, when he was speaking of the insulin, he meant direct shock supplied directly to the cortex. Someone else might compare that with some other system as regards the business of stimulating tissues, slices and so on. Lilly and Hughes, who have been working the past year pretty avidly, find that any pulse longer than 20 or 30 microseconds is damaging. Part of the theory is that it produces a movement or other dislocation of proteins. But all of these experiments that I know of are open to that criticism. Now, it is equally hard to accept Lilly and Hughes' conclusions on the basis of time intensity curves with the marked differences between C-fiber parameters and A-fiber parameters. But the experimental results seem to be quite clear at this time that they cannot electrolyze tissuesthat has been confirmed in other laboratories now-stimulate with short pulses, and keep the threshold down for weeks and apparently get all of the effects that Don Phillips and everyone else who has worked on the cortex have described.

They can do it more faithfully and more consistently and more accurately and without injury.

Dr. Streicher (National Institute of Mental Health): Dr. Grenell observed that the concentration of ATP was elevated during anesthesia, confirming the earlier findings of Lepage. Also an increase in ATP was observed after electroshock, suggesting that both experimental procedures result in a decreased utilization of available energy. Similiarly, in tracer studies with $\mathrm{P}^{32}$, Dawson and Richter reported that both pentobarbital anesthesia and electroshock decreased phosphate exchange in the phospholipid and nucleoprotein fractions of mouse brain. Low levels of creatine phosphate and ATP are observed after drug-induced convulsions but there is little unequivocal evidence that seizures were the direct result of decreased energy production by the brain.

Recent work by Bloom et al. with glucose labeled in the 1 or 6 position indicates that brain slices do not derive energy by the direct oxidation of glucose through the Warburg-Christian-Dickens pathway. However, evidence for the existence of an alternate pathway was presented some time ago by Barker, Shorr, and Malam in brain slices and by Himwich et al. in rivo.

Finally, it may be noted that there are two conflicting bodies of evidence concerning the effects of stimulation on phosphorylation in brain, peripheral nerve, and brain mitochondria. Dr. Abood reported that metrazol convulsions, electrical stimulation of nerve, and the passage of electrical pulses through mitochondrial suspensions all produce a decrease in phosphorylation. Opposite results have been observed in both brain and nerve, and McIlwain has not been able to reproduce the effects on mitochondria.

DR. LiNG: On the assumption that the nerve and muscle have something in 
common-that both of these tissues exhibit electrical activities-some experiments we have done might throw a little bit of light on this problem. What would happen if you inhibit the glycolysis and then inhibit the respiration completely with almost $100 \%$ nitrogen? Under these conditions, after several hours of treatment, the muscle obviously has lost its respiratory activity as well as its mechanical activity. The question arises; where are the possible sources the cells are still endowed with, since under such conditions if you keep the cells at zero degrees they maintain not only their normal ionic composition, but a full capacity to do electrical as well as mechanical work. Now, under such conditions, we know there are three possible sources of energy; that is, with the respiration and glycolysis both blocked. These sources are, of course, ATP and creatine phosphate as well as unknown sources. There is a possibility of checking and finding out what these unknown sources are. Some years ago Dr. Hill and his colleagues did make measurements of the total energy that could be liberated from such a system from the time the muscles were fully poisoned to the time the muscles completely died, and these measurements showed the total energy measured, since heat was very constant. This was later confirmed, and was of the magnitude of about 0.42 calories per gram. I may have to change the exact figure later, but it is constant per gram of wet weight. At that time the free energy data were not complete nor was the exothermic reaction involving the phosphorylation completely understood. So at that time the calculation of Hill and his co-workers did not lead to the final conclusion that the energy as measured by heat was completely liberated from A'TP. They, therefore, were suspicious of other pathways. Now, however, with our better knowledge of the various free energy methods and so on, you can recalculate the results and still come out with a value that is comparable; that is, a value measured as heat was completely explainable in terms of the energy-I mean, of the heat that could be generated from the total amount of ATP present. In other words, we concluded, from such a rough calculation, that there was no third pathway; there was no third source of energy. If you take this conclusion as a starting point, since the muscle was capable of maintaining its normal electrical and other properties in doing mechanical work, among the two sources of energy, ATP and creatine phosphate, there must be one that is completely responsible for this performance. Of course, it goes back to the ATP problem that Dr. Grenell has just brought up.

Dr. McElroy: Dr. Grenell and I agree perfectly that the narcotics are probably depressing the phosphate turnover. As a matter of fact we have done quite a bit of work on the mitochondria in the brain to show that we can get uncoupling with the concentration of compounds that he has used to show an increase in the ATP concentration in the intact animal. So one is led to the conclusion that the narcotic must be depressing utilization of the ATP through some unknown reaction and at the same time depressing the rate of its forma- 
tion. But the overall, steady state effect gives a net increase in the ATP concentration. One can do a pilot experiment on oxidative phosphorylation which shows the type of trouble one can get into when you do $\mathrm{P} / \mathrm{O}$ ratios. In oxidative phosphorylation one stimulates the system by adding glucose and hexokinase. This means that you can then pick up very sensitively any decrease in net production of ATP by measuring phosphate uptake. If, however, you leave out hexokinase and glucose and detect ATP formation directly, than it takes much larger concentrations of narcotics to give you a reduction in the ATP concentration in your preparations. This merely reflects again the concentration of the receptor systems.

There is one other thing, in this work we are talking about molecular structure and there is, as you know, a whole host of data from various sources indicating certain physical-chemical relationships between structure and biological activity. If oxidative phosphorylation is important as at least one site of action of the narcotics, one might expect to find the same physico-chemical relationships with mitochondria as with the whole organism. What we have been trying to do is to take some of these unique narcotics, namely, those whose partition coefficients decrease with a rise in temperature and those whose physiological effects decrease with a rise in temperature and determine their effect on oxidative phosphorylation at different temperatures. If one starts out with a concentration of salicylamide which gives $50 \%$ uncoupling at $15^{\circ}$, one finds that there is no uncoupling at $35^{\circ} \mathrm{C}$, whereas with chloretone the inhibition of the $\mathrm{P} / \mathrm{O}$ ratio increases with a rise of temperature. There is one other property which Johnson emphasized and that is the reversing of narcotic effect in tadpoles by high pressure. One can also show a reversal of inhibition of phosphate uptake by barbiturates by high pressure. Therefore, in terms of molecular structure and activity one can find in the mitochondria the same physico-chemical relationships. But $\mathrm{I}$ think it is quite clear from the analysis in the intact animal that this is not the only explanation for the mechanism of action of the narcotic. There is, certainly, something else going on on the other side and what this is, I think, is anybody's guess.

Chairman Gerard: Dr. Fischer, would you like to reopen your paper?

DR. Fischer: I would like to answer some of the still-open questions.

I would like equally to emphasize the importance of the forces of attraction and those of the binding of substances to wool. Remember, as an example, that you cannot kiss effectively if you do not embrace a person well. What is not generally recognized is that the attraction of a basic dye is stronger the more basic the dye (electrostatic effect) but the binding which takes place once the dye is attracted is generally believed to be due mainly to hydrogen bonding.

I am not so much concerned about the fact that according to some authors 
neurokeratin has not got the constitution one thought it had, just as I am not disturbed about the fact that the chemical configuration of some compounds does not explain how the specific pharmacological activity of those compounds is brought about. The main concern of my model experiments is that in many instances there is a relation between the affinity of a substance to wool and its biological activity. Of course, a model is always only a model and is able to give answers only to a restricted amount of questions.

Dr. Morales asked me which type of bonds are involved in the binding to wool of some compounds. I would like to say that the bond responsible for the affinity is likely to be mainly the hydrogen bond.

Let me illustrate another point. According to the Figure:

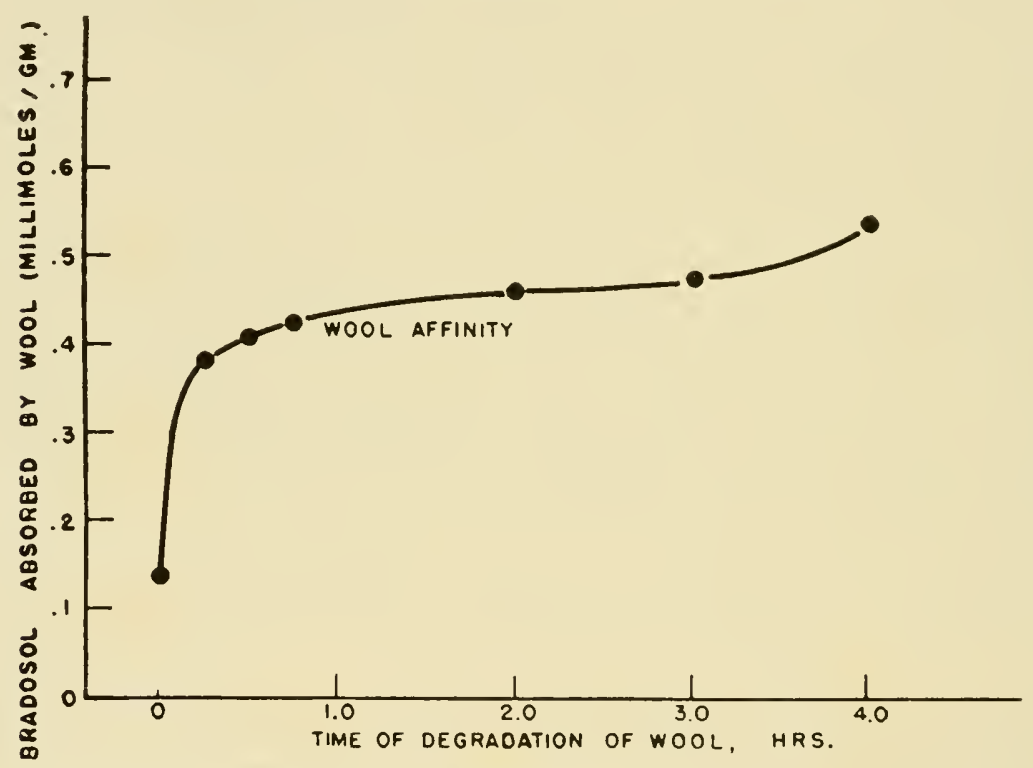

prolonged alkaline degradation of wool increases the affinity of a cationic compound, e.g. that of the quaternary ammonium disinfectant Bradosol for wool.

\begin{tabular}{l|l|l|l|l|l|l|l|l|l|l|}
\hline & \multicolumn{1}{c}{ Affinity of Bradosol for Wool Which Has Been Degraded with $0.15 \mathrm{~N}$} \\
$\mathrm{Na} \mathrm{CO}_{3}$ for
\end{tabular}

However, alkali clegraded wools, when treated, e.g., with diluted acetic acid, can be converted again into wools behaving practically like intact wool, i.e., 
displaying a low affinity for Bradosol. This example emphasizes the importance of the pretreatment of proteins prior to an affinity experiment.

CiIAIRIAN GERARD: Thank you. I am sure that does answer some of the questions that were going around, if not all of them. Dr. Fischer mentioned that he had tried a number of other materials than wool. I didn't remember whether he mentioned silk.

Dr. Fischer: I did.

Dr. GERARD: Does that not act the same?

DR. FISciIER: One of the differences between wool and silk is that silk is a crystalline protein, whereas wool is a fibrous protein. Intact, degummed silk stains a deep violet when stained with crystal violet, whereas intact wool does not take the stain, i.e. it is gram-negative.

CHAIRMAN GERARD: Have you by any chance had a chance to study the wools from sheep that have been raised on a copper deficient diet or manganese diet in which you get changes in the wiggliness and in the color and so on, or abnormal protein molecules?

Dr. Fischer: No.

Cilairman Gerard: It might be a possible lead for you.

DR. MOR ALEs: With respect to the other proteins I think the main difference between silk and keratin would be the number of polar groups and silk is notoriously low, so I think if you wanted to try a different protein you ought to try one that is not in the same series.

The reason $I$ had asked you about the type of binding is that, if my arithmetic is correct, you have something like one molecule bound per ten. That means that you must have pulled an awful lot of this stuff into it.

Now, if you say this is due to hydrogen bond or due to some electrostatic attraction, you class this as a rather loose binding and this seems a little contradictory to me.

Dr. Fischer: You have $0.2 \mathrm{gm}$. of substance dissolved in $50 \mathrm{cc}$. of distilled water and one gm. of wool; this means that $20 \%$ of substance was taken, calculated on the basis of the weight of the wool. The substance therefore is present as a surplus. After 10 minutes at $90^{\circ} \mathrm{C}$. and $\mathrm{pH} 5.2$ equilibrium has been reached and the affinity experiment is completed by rinsing the wool three times with distilled water at room temperature. Then we believe that the substance is bound to the wool mainly by hydrogen bonds.

Dr. Morales: How do you know this?

Dr. Fischer: I do not know, I only assume, but I am in good company with my assumption as H. R. Chipalkatti, C. H. Giles, and D. G. M. Vallance (Journal Soc. Dyers a. Color. $70:(1954), 4375)$ believe the same after determining the adsorption of organic compounds by wool, silk and nylon from aqueous and non-aqueous solutions. 
Dr. Morales: If it is merely hydrogen bond you ought to be able to take this out.

DR. Fischer: I would be able if I would desorb the wool for perhaps three or four days. You can take off, i.e. desorb every substance; it is only a question of time, temperature, $\mathrm{pH}$. etc. There are certain substances which are so firmly bound to wool that to desorb them would considerably alter the chemical as well as physico-chemical structure of the wool protein.

Dr. Morales: We are talking about hydrogen bonding, though; this is something that you could easily reverse.

Dr. Fischer: I say, mainly of hydrogen bonds but I do not say that these are the only bonds involved.

Dr. Blum: It seems to me that you do this at $90^{\circ} \mathrm{C}$. first and then you wash it at room temperature. I think Dr. Morales is correct in saying that he would expect a pretty rapid charige if it was just hydrogen binding. But, on the other hand, in the original experiment you bind this enormous quantity at $90^{\circ}$, in which situation, the bond structure of the keratin is quite stabilized and then you reduce it to $25^{\circ}$. You may bind up your substances by much more semi-covalence as you may have multiple entrapped, enclosed molecules. You might easily account for this large quantity and yet you would also be able to explain why it didn't come off.

Dr. Fischer: Let me give you one experimental detail which refers to both of your remarks.

If you determine the absolute affinity of a compound for wool, there is no rinsing involved at the end of the procedure; these absolute values are 5-6 times higher than those obtained by the weight increase method where rinsing is applied at the end of the procedure. 


\title{
III
}

\section{MEMBRANES OF NEURONS}

\section{The Structure of Nerve Cell Membranes}

\author{
L. J. Mullins
}

Biophysical Laboratory, Purdue University, Lafayelle, Indianal

I NVESTIGATIONS OF MEMBRANE STRUCTURE must contend with the fact that it is not technically possible to demonstrate a structure by optical methods, and that the elements of the structure cannot be isolated in a pure form for chemical analysis. An indirect approach, involving an examination of those molecules that produce physiological effects by their interaction with the membrane, appears to offer certain advantages in membrane structure analysis, and forms the subject of this discussion. Interaction between the membrane and certain molecules is presumably the result of a structural complementarity, hence if enough different molecules acting on the same membrane are examined, structural information can be obtained. The methods used in this study involve first, the setting up of a model of the membrane; the presumed physical behavior of the model may be compared with the results of experiment in order to decide upon further improvements in the model. An early model of the membrane (Davson and Danielli, 1943) suggested that the site of diffusion resistance was a bimolecular lipid layer. Aside from the difficulty in seeing how such a structure, between two aqueous phases, would remain stable, experiments of several sorts have suggested a revision of the model. When measurements of the rate of penetration of water, using a $\mathrm{D}_{2} \mathrm{O}$ gradient, were compared with penetration rates under an osmotic gradient, the latter were considerably higher (Hevesy et al, 1935; Prescott and. Zeuthen, 1953). This behavior is characteristic of pores rather than a solution process in a non-aqueous medium, as first shown by Kofoed-Johnsen and Ussing (1953). A second difficulty arises when one attempts to alter the ion permeability of a lipid layer to conform with recent studies on the action potential. The gross structural rearrangement (Danielli, 19+1) required, appears incompatible with the constant membrane capacity

${ }^{1}$ Aided by a grant (B-139) from the National Institute for Neurological Diseases and Blindness, Bethesda, Md. 


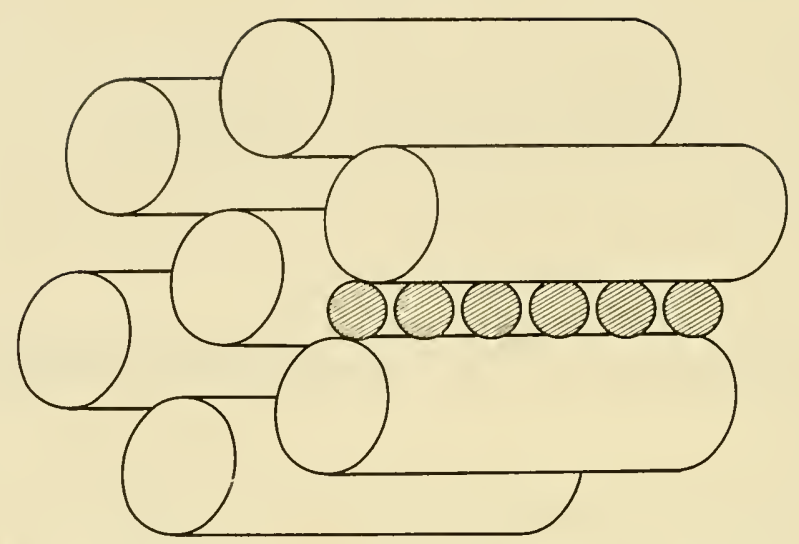

FIg. 1. Macromolecular cylinders, representing structurai elements of the cell membrane, are shown in hexagonal packing. The spacing between the molecules has been greatly expanded to show the membrane pore that is formed between any three cylinders, and molecules are shown passing through one pore. The length of the cylinders is the membrane thickness, and the diameter a function that sets the pore size.

(Curtis and Cole, 1938) observed during activity. Finally, a lipid film membrane cannot show the specificity of interaction with certain molecules that is observed, while a pore membrane, as will be shown later, is able to discriminate between molecules of identical lipid solubility.

If one assumes that the evidence favors a membrane with pores, two general types may be considered: (a) the random polymer network, a structure of fibrous molecules that are cross-linked so as to yield pores of widely varying size and shape, and (b) the oriented macromolecular liquid membrane, a structure in which the pores are formed by the inability of macromolecules, in their most efficient packing, to fill all the space in the liquid. The simplest example of (b) is that of macromolecular cylinders in regular hexagonal packing; the pores exist at the junction between any three cylinders and are characterized by a regularity of size but, as will be shown later, can show a considerable degree of specificity in their interaction with molecules of various sorts. Fig. 1 shows the suggested arrangement for a membrane structure.

\section{Ion Transfers Across the Membrane}

Because much of our present understanding of the action of the membrane has come from studies of the transfer of ions across it, certain structural information can presumably be derived from such work. In order to apply the results of ion transfer studies it is necessary to know something about the properties, and more especially the sizes of the ions concerned in nervous excitation. The suggestions that the permeability difference of the resting membrane to $\mathrm{Na}^{+}$ 
TABLE I

\begin{tabular}{|c|c|c|c|c|}
\hline \multirow{2}{*}{ No. of Layers of Hydration } & \multicolumn{2}{|c|}{$\mathrm{Na}^{+}$} & \multicolumn{2}{|c|}{$\mathrm{K}^{+}$} \\
\hline & Radius of Ion, Å. & $\begin{array}{l}\text { Hydration Energy } \\
\text { in kcal / mole }\end{array}$ & Radius of Ion, $\AA$. & $\begin{array}{l}\text { Hydration Energy } \\
\text { in kcal/mole }\end{array}$ \\
\hline $\begin{array}{l}0 \\
1 \\
2 \\
3 \\
+ \\
5-x\end{array}$ & $\begin{array}{r}0.95 \\
3.67 \\
6.39 \\
9.11 \\
11.83\end{array}$ & $\begin{array}{r}49.8 \\
19.0 \\
7.7 \\
4.1 \\
13.9 \\
94.5\end{array}$ & $\begin{array}{r}1.33 \\
4.05 \\
6.77 \\
9.49 \\
12.21\end{array}$ & $\begin{array}{r}34.5 \\
16.3 \\
6.9 \\
3.9 \\
13.4 \\
75.0\end{array}$ \\
\hline
\end{tabular}

Based on values for crystal radii of Pauling (1940) and heats of hydration of Verwey (1942). I am indebted to Dr. R. L. Platzman for this calculation.

and $\mathrm{K}^{+}$results from a larger "effective" size of the $\mathrm{Na}^{+}$in aquecus solution, and that special chemical agents or "carriers" are necessary for the selective increases in $\mathrm{Na}^{+}$or $\mathrm{K}^{+}$permeability that occur in the active membrane are to be contrasted with very different proposals, to be developed below, that for pores of a size $+-5 \AA$. in radius, $\mathrm{Na}^{+}$and $\mathrm{K}^{+}$have precisely specified sizes and will only penetrate through pores that closely fit the ion. These notions are arrived at by considering the forces acting on the water structure in the vicinity of an ion.

In Table I is shown the extent to which various layers of hydration are bound to $\mathrm{Na}^{+}$and $\mathrm{K}^{+}$together with values for the sizes of the resulting hydrated ions. The importance of these data lies in two points: first, neither of the ions can have a size that does not represent some integral number of water-molecule layers, and second, the energy with which water molecules are bound in the first hydration shell of six molecules is so great that the water is in essentially a non-deformable solid state, while the energy with which the second water shell is held is still much greater than thermal energy. The sizes of ions with 3 or more shells of hydration are so very large compared with probable membrane pore sizes that they need not be considered further.

The potentialities for ion penetration through a membrane pore would appear to be such that if an ion at a specified level of hydration closely approximates the size and shape of a pore, it may exchange all its hydration, beyond the specified level, for the solvation afforded by the walls of the membrane pore. If the pore is circular in cross-section, then only ions of 1 or 2 layers of hydration need be considered because these are the only hydration arrangements that yield spherical ions. If pores have a somewhat different shape, it is possible to consider ions that have one complete hydration layer and a partial second layer. 
There are still, however, only a limited number of such arrangements because of the small number of water molecules comprising the second layer of hydration. If $\mathrm{K}^{+}$approaches a pore that is precisely the same size as this ion with its first hydration shell (denoted $\left(\mathrm{K}^{+}\right)_{1}$ ), it may, as indicated previously, exchange hydration, for water shells from 2 to infinity, for a similar attraction with the structure lining the pore. If the pore is somewhat smaller than $\left(\mathrm{K}^{+}\right)_{1}$, penetration cannot occur for steric reasons, while if the pore is somewhat too large, penetration likewise cannot occur because the attraction of the ion for water shells of 2 and greater is not compensated by a solvation of similar magnitude in the pore. Such solvation demands a close fit between the ion and the pore wall.

Since a collection of pores formed by cylindrical macromolecules undergoing thermal motion may be expected to show a dispersion in size, we have in this model a mechanism for discriminating between $\mathrm{Na}^{+}$and $\mathrm{K}^{+}$that resides solely in the size of the ion. Sodium ion is usually considered to be larger than $\mathrm{K}^{+}$, because its larger energy of hydration results in a lower mobility in aqueous solutions; in the present discussion it suffices to note that while the difference in hydration energy for shells greater than $\left(\mathrm{Na}^{+}\right)_{2}$ or $\left(\mathrm{K}^{+}\right)_{2}$ is $1 \mathrm{kcal} / \mathrm{mole}$, and the difference between $\left(\mathrm{Na}^{+}\right)_{1}$ and $\left(\mathrm{K}^{+}\right)_{1}$ is $5 \mathrm{kcal} /$ mole, a similar enhancement in pore solvation energy may be expected for $\mathrm{Na}^{+}$. Because the crystal radius of $\mathrm{Ca}^{++}$is the same as that for $\mathrm{Na}^{+}$, these ions will have very close to the same size at all levels of hydration, hence $\mathrm{Ca}^{++}$can be considered as a competitor for $\mathrm{Na}^{+}$pores. Values for other ions are: $\left(\mathrm{Mg}^{++}\right)_{1} 3.37,\left(\mathrm{Mg}^{++}\right)_{2} 6.0,\left(\mathrm{Cl}^{-}\right)_{1} 4.56 \AA$. radius.

In order to account for the resting potential of axons under a variety of conditions, Hodgkin and Katz (1949) have proposed that the permeabilities of ions concerned with the potential stand in the following ratios: $\mathrm{P}_{\mathrm{K}}=$ $1.0, \mathrm{P}_{\mathrm{Na}}=0.04$, and $\mathrm{P}_{\mathrm{Cl}}=0.45$. Since the permeability of the membrane to an ion is a function of the product, partition coefficient $\times$ mobility, we might, for the moment, make the simplifying assumption that all three ions are of equal mobility in the membrane, and that the number of pores of an appropriate size to fit a particular ion is equivalent to the partition coefficient of this ion. In Fig. 2 we have plotted the normal distribution curre that is specified by the permeabilities and radii of $\left(\mathrm{Na}^{+}\right)_{1},\left(\mathrm{~K}^{+}\right)_{1}$, and $\left(\mathrm{Cl}^{-}\right)_{1}$. The assumption has been made that a 'good fit' for ion solvation purposes is $0.05 \AA . \pm$, although some permeability data on the red cell where $\mathrm{Rb}^{+}$competes with $\mathrm{K}^{+}$suggest that $\pm 0.1 \AA$ might be a better value. The question of the extent to which the membrane potential is dependent upon a particular value of mean interspace size, can be answered by shifting the distribution curve to larger and smaller mean values and computing the partition coefficient for each of the ions as well as the expected membrane potential, using the figures for squid axon (Hodgkin and Katz, 1949). The results of such calculations are shown in Fig. 3 and indi- 


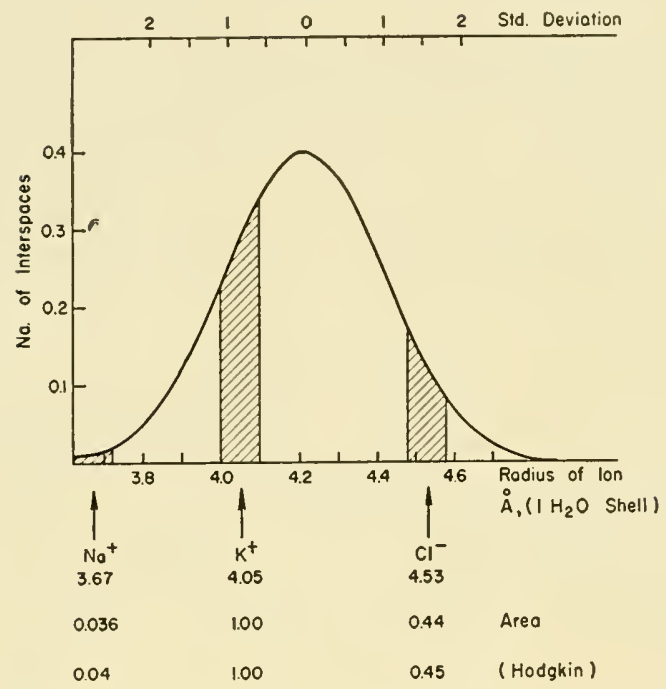

FIG. 2. The number of membrane interspaces is plotted against their size as measured by the radius of a circle that can be inscribed in the interspace. Values for ion sizes are from Table $I$, and the mean interspace size is set by assuming that the permeability of the various ions is proportional to the number of interspaces of a size of proper fit.

cate that, as might be expected, the ratios of ion partition coefficients vary widely when the interspace size clistribution is shifted, but the calculated potential is constant as long as the values for $\mathrm{Na}^{+}$are low.

Some revision of the model thus far developed is required in order to accommodate the following. If $\mathrm{Ca}^{++}$is in competition with $\mathrm{Na}^{+}$for interspaces, we may expect that in spite of its low concentration in Ringer solution or sea water, it actually occupies a significant fraction of $\mathrm{Na}^{+}$interspaces and that further, the mobility of $\mathrm{Ca}^{++}$in a membrane pore is much lower than that of $\mathrm{Na}^{+}$. In addition, the assumption of equal mobility for all singly charged ions must be examined, as well as the objections raised by Grundfest (1955) to the notion that the resting potential is specified by the ratio of $\mathrm{K}^{+}$or $\mathrm{Cl}^{-}$across the membrane. The difficulties stem from a demonstration that the alteration of internal $\mathrm{K}^{+}$or $\mathrm{Cl}^{-}$concentrations does not alter the potential, that injected cations block conduction in the order $\mathrm{Ca}^{++}>>\mathrm{Na}^{+}>\mathrm{K}^{+}$, and that injected $\mathrm{Ca}^{++}$depolarizes. Some of these objections can be met by assuming that the rate at which $\mathrm{K}^{+}$can be introduced into the membrane is subject to saturation at certain levels of concentration, that $\mathrm{Ca}^{++}$blocks $\mathrm{Na}^{+}$channels, and that increases in internal $\mathrm{Na}^{+}$concentration are likely to result in much increased $\mathrm{Na}^{+}$effluxes during activity.

In a recent paper, Hodgkin and Keynes (1955) have shown that some of the 


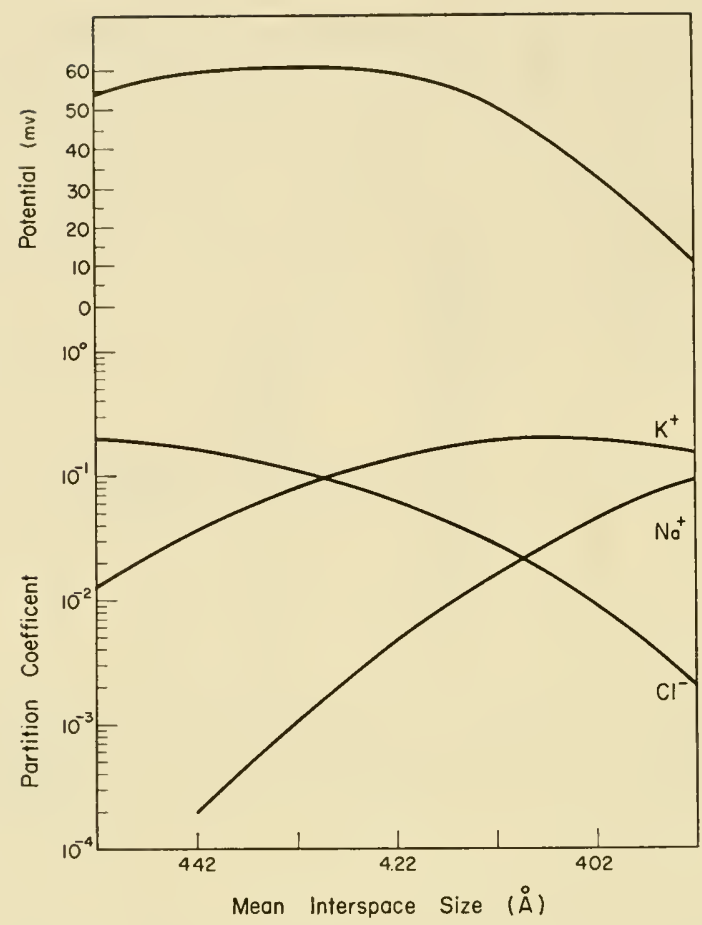

Fig. 3. The lower curves show the changes in the partition coefficient (number of interspaces that fit) of various ions as the mean interspace size of the membrane is varied. The curve at the top is the membrane potential computed on the basis that ion permeability and partition coefficient will vary proportionally.

anomalies of $\mathrm{K}^{+}$movement in axons can be explained if the ions move in single file through channels in the membrane, and that movement in one direction tends to inhibit movement of the same ion in the opposite direction. That this effect does not appear important for other ions might seem to suggest that the number of $\mathrm{K}^{+}$channels is small when compared with those for other ions, while permeability data indicate the reverse. An explanation of this coupling as well as that between membrane potential and electrical resistance, as noted by Jenerick (1953) for muscle can be obtained by considering the mechanism involved in the diffusion of $\mathrm{K}^{+}$through membrane channels. Assuming that an ion has been partitioned to a channel of proper size, its progress along the channel will be influenced by the radius at each point along the channel. Because the macromolecules that form the channel must be undergoing thermal motion, the channel must vary in size from one point to another and an ion at one end will only progress when it obtains energy from a collision at the same time that the channel size immediately ahead is within permitted limits. If, on the 
other hand, there are a number of $\mathrm{K}^{+}$in the channel and these are equally spaced across the membrane, the strong interaction between the ions and their solvation (the channel walls) prevents any fluctuation in channel size along its length. Through such a pore, ions can move without the barrier occasioned by random changes in channel size. The information presently available is inadequate for determining whether the membrane channel is comparable to a homogeneous dielectric, or whether there are ionized sites that can be occupied by an ion. In this latter case, the sites need not be permanently ionized but only ionizable upon the close approach of an ion. The actual situation is not important for the present discussion except to note that for a homogeneous dielectric, ions of like charge could not approach each other more closely than about $25 \mathrm{~A}$., so that for a $100 \AA$. membrane, the maximum number of ions per channel would be four. Potassium ion distributions resulting in low and high mobility are shown below. The lower line represents a "through channel" or one of high ion mobility and is only compatible with a diminished membrane

Inside

$$
\mathrm{K}^{+}+\ldots \ldots+\ldots
$$

Outside

$$
\mathrm{K}^{+}-\mathrm{K}^{+}--\mathrm{K}^{+}--\mathrm{K}^{+}
$$

potential, while the upper line represents a channel with low ion mobility and is the probable arrangement at high membrane potentials.

Since membrane potential and number of through channels are apparently inversely related, the passage of an electric current outward through the membrane may transform a number of $\mathrm{K}^{+}$channels into through channels; a similar effect is to be expected for $\mathrm{Cl}^{-}$moving in the opposite direction. The change to a through channel is equivalent to the imposition of a new interspace distribution pattern upon the membrane; the local and transient changes that are involved are of considerable interest. Since the through channel is a rigid structure, its creation may perturb the interspace size distribution for those channels immediately surrounding it. Some 12 interspaces have in common at least one of the macromolecules immediately surrounding a given interspace, but the disturbance of creating the through channel is likely to extend to at least another layer of membrane molecules. The general reaction to the rapid filling of a $\mathrm{K}^{+}$channel may be expected to be a momentary compression of these surrounding interspaces. Some will be transformed to $\left(\mathrm{Na}^{+}\right)_{1}$ size, thus permitting an influx of these ions; a considerable multiplication is to be expected if the creation of a $\mathrm{K}^{+}$through channel extends in effect over only two layers of membrane molecules as 36 new $\mathrm{Na}^{+}$channels may be created. As the permeability of the resting membrane $10 \mathrm{~K}^{+}$is considered as 20 times that of $\mathrm{Na}^{+}$. activity can result in a $720 \times$ increase in $\mathrm{Na}^{+}$if the mechanism for interspace size change is efficient. The influx of $\mathrm{Na}^{+}$will be terminated by the 'flow' or diffusion of membrane molecules forming the $\mathrm{Na}^{+}$chamnels to more probable 
values of size and, possibly, by the tendency of $\mathrm{Ca}^{++}$to plug the channels. The application of slowly increasing outward electric currents allows the membrane macromolecules to assume new positions with respect to the through channels being formed, without large increases in $\mathrm{Na}^{+}$flux because the rate of through channel creation and the rate of 'flow' of macromolecules may be assumed to be comparable. It might be expected that membranes would differ in what might be called their 'miscroscopic viscosity' or their resistance to flow or redistribution of their structural elements, and that this would be apparent as a difference in accommodation.

A variety of other arrangements for the transformation of the membrane from a relatively $\mathrm{Na}^{+}$impermeable-permeable structure suggest themselves but, in some way or another, appear less attractive than that described above. One might set the mean interspace distribution at the size of $\left(\mathrm{Na}^{+}\right)_{1}$, but consider that practically all these sites are occupied by $\mathrm{Ca}^{++}$at Ringer concentration. With $P_{\mathrm{Na}}$ properly adjusted, $\mathrm{P}_{\mathrm{K}}$ and $\mathrm{P}_{\mathrm{Cl}}$ could be given proper values. Outward current could then be thought of as removing the $\mathrm{Ca}^{++}$blocking the $\mathrm{Na}^{+}$channels and permitting the required inward $\mathrm{Na}^{+}$flux, but it is hard to see why this should not continue as long as the membrane is depolarized.

Further improvements in the model should consider that ions on both sides of the membrane will tend to distort the interspace size distribution from its mean value for an ion-free structure, to one with a mean close to the size of a particular ion. At the inner surface of the membrane the mean interspace size is probably close to that of $\left(\mathrm{K}^{+}\right)_{1}$, other cations or anions being relatively ineffective competitors because of their size or low concentration, while at the outer surface of the membrane $\mathrm{Na}^{+}$and $\mathrm{Cl}^{-}$compete but the actual mean may be expected to be close to $\mathrm{Cl}^{-}$in size. If, in the resting membrane, the partition coefficients are maximal for $\mathrm{Cl}^{-}$on the outside and $\mathrm{K}^{+}$on the inside, coefficients for the other ions at each surface can be obtained from a distribution curve and relative figures for the mobilities of ions moving in either direction can be given as shown in Table II.

In a non-through channel the possibility exists that ions of different size are alternately loaded owing to the continuous variation in channel size that is presumed to be taking place. This effect may be expected to result in very

TABLE II

\begin{tabular}{|c|c|c|c|c|c|}
\hline \multirow[b]{2}{*}{ Ion } & \multirow{2}{*}{ Permeability } & \multicolumn{2}{|c|}{ Outside Surface } & \multicolumn{2}{|c|}{ Inside Surface } \\
\hline & & Partition coefficient & Nobility & $\begin{array}{l}\text { Partition } \\
\text { coefficient }\end{array}$ & Mobility \\
\hline $\mathrm{K}^{+}$ & 1. & 0.01 & 100 & 0.2 & 5 \\
\hline $\mathrm{Cl}^{-}$ & 0.4 & 0.2 & 2 & 0.02 & 20 \\
\hline $\mathrm{Na}^{+}$ & 0.05 & 0.00005 & 1000 & 0.02 & 3 \\
\hline
\end{tabular}


low mobilities for the ions concerned. If two ions of the same size and opposite charge are alternately loaded into a channel, it would appear that the ions in the channel would escape the influence of the electric field imposed by the membrane potential and move outwards by diffusion forces alone.

\section{The Action of Narcotic Agents}

In a previous study of narcosis (Mullins, 195t) it was suggested that the experimental data could be explained equally well by representing the membrane as a homogeneous lipid film or as an aggregate of pores formed by macromolecules since, in either case, a constant thermodynamic activity for narcosis implied that the narcotic molecules fitted into a structure at their site of action as they did into their own pure liquids (Brink, 1951). Some of the reasons for believing that the membrane has a pore structure have already been presented, and others will be given later in the discussion. The size of such membrane pores or interspaces can be estimated in the following way. Data for the narcosis of organisms by homologous series of paraffins, alcohols or ethers quite frequently show that the threshold for narcosis expressed as thermodynamic activity is constant from member to member up to some point, and then rises such that $\log$ activity vs. number of carbon atoms are linearly related. The point where a discontinuity in activity vs. size of narcotizing molecule takes place may be interpreted as indicating some limitation imposed by the membrane on the uptake of molecules of larger and larger size. If all membrane interspaces were the same size, the discontinuity ought to be abrupt, while it is usual to find increasing activities required for molecules with 5 to 10 carbon atoms. Since we are dealing with molecules with an alkyl chain of increasing length, it is possible to adopt the view that the size of the molecule in a particular orientation (with the chain perpendicular to the surface of the membrane) is not changing, but only the concentration of molecules in a particular orientation. Somewhat more certainty with respect to size attends the use of rigid molecules as narcotics. Thus benzene has a threshold for narcosis for the grain weevil of 0.02 , while methyl benzene has a threshold of 0.08 (Ferguson, 1951). We can specify a size for both molecules and consider that the difference in concentration is because the methyl group on toluene imposes on this molecule a limitation on penetration not found in benzene. The rigidity of both molecules makes it likely that the nature of the limitation is an orientational one. Thus, toluene can pentrate in an orientation where its methyl group does not interact with the walls of the interspace but as the number of such orientations is only a fraction of total number of collisions with the surface, the concentration of toluene must be increased for narcosis. We may expect, therefore, that for paraffins there are relatively few interspaces larger than benzene. When molecules that are practically spherical are used as narcotics, it is somewhat easier to analyze the situation; they have the same profile in all orienta- 
tions. Such substances ought, in theory, to be ideal for determining interspace size but certain practical considerations make this not so. The first difficulty is the quantized values of the diameter; in the series $\mathrm{CH}_{4}, \mathrm{CCl}_{4}, \mathrm{CBr}_{4}$, and $\mathrm{CI}_{4}$ the diameters increase in large steps and so does the intermolecular attractive force of the compounds. This is equivalent to saying that the affinity of the halogens of high electron density may compensate for the diminution in the number of interspaces into which the substances may fit.

The general features of the model for the membrane permit an understanding of the mechanism of narcosis by molecules that are small in comparison with the mean interspace size. The increase in $\mathrm{Na}^{+}$permeability taking place during excitation requires that a large number of channels be unoccupied while the narcotic will tend to occupy channels at random and thus to diminish ion fluxes taking place during activity. The resting ion fluxes need not be proportionately affected; indeed, as will be shown subsequently, narcosis by molecules that are greater in size than the mean of the interspaces is capable of taking place with various effects on ion transfers.

\section{Specific and Non-Specific Receptors}

While it is generally agreed that a variety of molecules of different sorts can cause narcosis, that the concentrations necessary are predictable from the physical parameters of the molecule, and that narcosis appears to be an equilibrium phenomenon, no such agreement is to be found when the capacity of a molecule to initiate nervous excitation is to be considered. Rather, it is conventional to postulate the existence of receptors on the cell surface that are responsible for the observed excitatory (and sometimes inhibitory) specificity of particular chemical compounds. Because receptors are situated very close to the membrane, it is reasonable to inquire how one distinguishes between these two structures; the difference lies mainly in the fact that the membrane is not supposed to detect chemical specificity. If, therefore, it can be shown that the membrane model can detect small differences in molecular structure, we can dispense with the concept of recepters and incorporate their features into the membrane model.

One of the most versatile sets of receptors is the olfactory epithelium of man and a study of the threshold for the detection of various substances may serve as an introduction to what might be called non-specific receptors. Results of threshold studies for homologous series of paraffins, chloroparaffins and alcohols show that (a) the threshold is about the same for any of these three compounds at the same number of carbon atoms, and (b) stimulation by these substances is strongly dependent upon the size of the molecule ( $\mathrm{C}_{4}$ being about optimal and 50 times better than $C_{1}$ or $C_{8}$ (Mullins, 1955a)). As, in the paraffins, there is no reactive chemical grouping, it appears necessary to postulate that it is the size of the molecule that limits its stimulating effectiveness. It is also an 
experimental fact that compounds from $\mathrm{C}_{1}$ to $\mathrm{C}_{5}$ are all equally good as general anesthetics when concentrations for anesthesia are expressed as thermodynamic activity. The observations mentioned about are best explained if (a) excitation is a time-dependent process and one that requires a close fit between stimulating molecule and membrane interspace, (b) there is some dispersion in the size of interspaces in the membrane. Both these suggestions, in slightly different form, have been used to explain changes in ion permeability of the membrane and it therefore seems likely that the filling of a membrane chamel with organic molecules that fit closely, initiates the same transitory increase in $\mathrm{Na}^{+}$influx as that produced by $\mathrm{K}^{+}$. At the top of Fig. 4 is shown the situation with respect to fit for molecules of various sizes. Very small molecules not only may expect to find few sites that fit, but will be distributed almost uniformly throughout the membrane sites that are shaded in the diagram and indicate sites where occupation results in narcosis. With very large molecules the situation is quite different, the fact that most channels in the membrane are too small restricts the distribution of such molecules to a few sites, and to even fewer that are larger still. At the bottom of the diagram is shown the total number of sites existing at various levels of interspace size, and the ratio of number of sites that fit a molecule (within 0.5 standard deviation) to the total number of sites. Clearly as molecular size increases so does the probability that the molecule will occupy a channel that it fits. Stated another way, as molecular size increases so does the specificity of the membrane with respect to the shape of the molecules that it will admit.

If, instead of considering paraffins, and spherical molecules, we have irregularly shaped molecules with strong polar groupings, a very large number of further complications are introduced. The nature of these difficulties can only be hinted at; they involve dealing with the problem of what fraction of the molecules arriving at the membrane are properly oriented for penetration, of the penetrating molecules how many are properly oriented for binding to specific points within the interspace, whether reorientation can take place within the interspace, and the estimation of the binding strength and chemical stability of molecules in the membrane. The halogenated insecticides provide a convenient transition from ideal molecules to the intensely complicated situation with highly polar molecules.

A remarkable series of compounds exist in the isomers of hexachlorcyclohexane (sometimes called benzene hexachloride or BHC). They result from interchanges between chlorine and hydrogen on a cyclohexane ring, and are, in effect, changes in the shape of the molecule without changes in the total number of atoms. Only one isomer, the $\gamma$, is a strong convulsant both in insects and mammals, and one isomer, the $\delta$, has appreciable narcotic potency. It has been possible to explain this peculiar behavior on the basis of the model of the membrane, with the additional assumption that convulsant activity is caused 

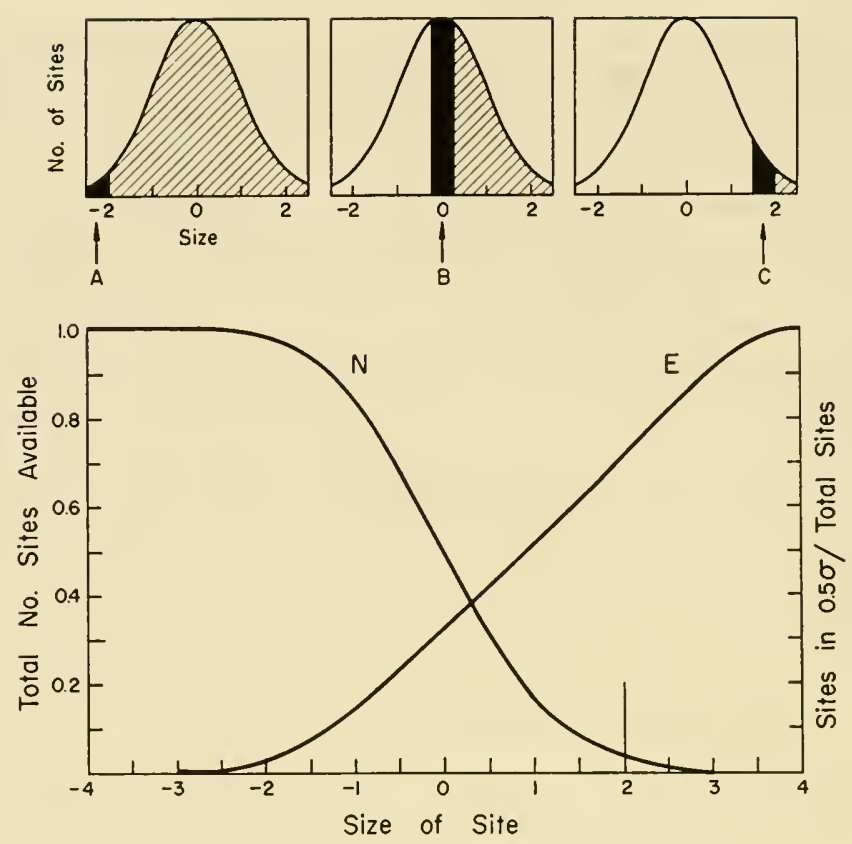

Fig. 4. The three curves at the top show the number of molecules that fit an interspace within \pm 0.25 standard deviation (black bar) and the number of molecules that are too small for fit (shading) for a small molecule (A), a medium sized molecule (B), and a large molecule (C). Below, the total number of sites available to molecules of various sizes is shown by curve $\mathrm{N}$; if narcosis requires $4 \%$ of all sites to be occupied, the line at 2 standard deviations represents the upper limit in size for narcotics. The curve $\mathrm{E}$ represents the ratio of the number of sites that fit a molecule corresponding to a particular site size, to the total number of sites that can be occupied. Curve $\mathrm{E}$ is a measure of the excitatory potential of a molecule.

by a local crystallization or phase transformation taking place in the membrane as a result of the strong interaction between the $\mathrm{Cl}$ atoms of the $\gamma$ isomer and the surrounding membrane molecules (Mullins, 1955b). Such a local transformation of a small part of the membrane cannot readily be compensated for because rearrangement of nonaffected membrane molecules cannot restore the original interspace distribution without creating further disturbances. The change involved appears to be of a totally different order of magnitude from that produced by filling interspaces with $\mathrm{K}^{+}$, and is in the nature of a marked enlargement of the surrounding interspaces. It appears consistent for the change to result in the leakage of $\left(\mathrm{Na}^{+}\right)_{2}$ of radius $6 .+\AA$. The general instability at the site of insertion of $\gamma$ - $\mathrm{BHC}$ appears such that the membrane will be undergoing random fluctuations in permeability to all ions, e.g. an electrical short circuit will develop from time to time. From steric considerations it can be shown that 
no other isomer can be oriented in the membrane interspace in such a way as to provide the $\mathrm{Cl}$-membrane interaction present in the case of the gamma isomer. The narcotic effects of the $\delta$ and other isomers can be explained on the basis of solubility and non-specific interaction. It would appear that the orientation of a molecule in a membrane interspace may be of decisive importance for chemical excitation and that an interspace without any assumed chemical specificity is able to discriminate between closely related compounds and is able, thereby, to be variously affected.

Another molecule, DDT (2,2 bis (p-chlorphenyl) 1,1,1, trichloroethane) can be shown not to penetrate except in one particular orientation. The five chlorine atoms in the molecule are not considered to bind at specific points, but as in BHC they serve to intensify the London-van der Waals forces between the molecule and the groupings in the interspace. Since the interspace is presumed to be of the order of $100 \AA$. long (the membrane thickness) somewhat more than 20 DDT molecules are required to fill it. There are a rather large number of homologs of DDT only a very few of which have any action at all comparable as convulsants; these compounds, as well as the $\mathrm{BHC}$ isomers, provide a rather critical test of the lattice defect mechanism of action already suggested because the membrane model must clearly reject inactive compounds and accept the active one. In Fig. 5 is shown DDT and some homologs oriented in the membrane interspace. This is the contracted size of the membrane interspace and the actual size of the unoccupied interspace would be larger. The membrane phase change suggested as an explanation for the mechanism of action of DDT also affords an understanding of the anomalous negative temperature coefficient of DDT action (Lindquist et al, 1945; Potter and Gillham, 1946; Hafliger, 1948). With macromolecules of the sort suggested as components of the membrane, a sharp phase transition with temperature can hardly be expected for the membrane-DDT complex; melting will, however, become more and more probable as the temperature is raised. Increased excretion or detoxification appears ruled out as an explanation for the effect (Vinson and Kearns, 1952) as does the suggestion of Munson (1954) that lipid depots in insects compete more effectively with receptors for DDT at elevated temperatures. Such an explanation is hardly compatible with the observations (Welsh and Gordon, 1947) on the essentially irreversible binding of DDT that is to be expected if it forms a membrane-DD'T complex.

The excitatory action of DDT can be antagonized by certain anesthetic agents but an hypothesis that the anesthetic blocks those enlarged interspaces around a DDT site cannot be entertained because there is no way to compel the anesthetic molecules to select such sites from all others offered by the membrane. Two types of anesthetic action are distinguishable from the model; in the first, small molecules occupy interspaces of all sizes equally well so that 

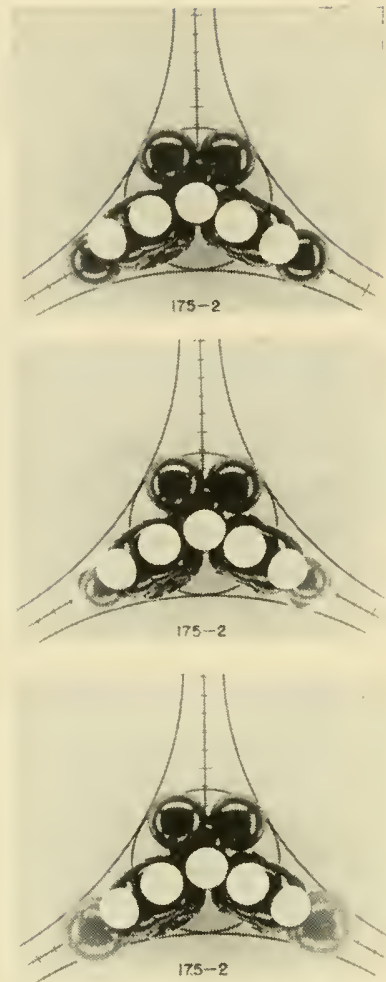
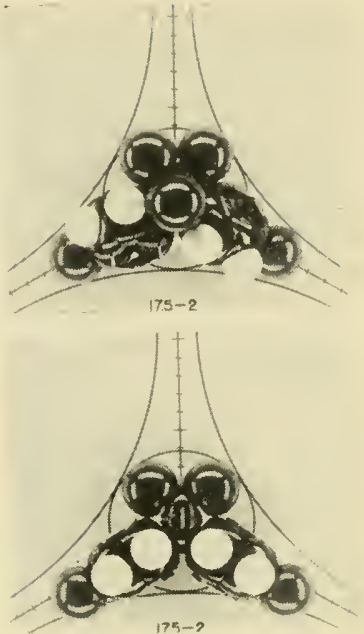

$175-2$

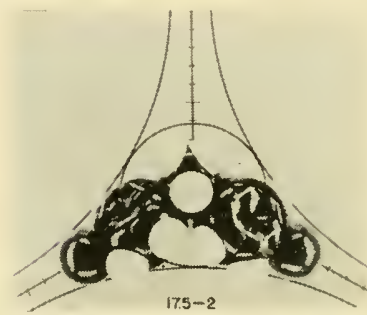

FIG. 5. The fit of DDT and several homologs into an interspace formed by 3 membrane molecules of radius $17.5 \AA$. and a separation of $2 \AA$ is shown. The grid in the interspace is ruled in units of $1 \AA$, and a circle is inscribed to denote the limit of size of molecule that can penetrate in an orientation independent manner. In the left column, from top to bottom, DDT, Methoxychlor (p-methoxy DDT), and 2,2 bis (p-iodophenyl) 1,1,1 trichloroethane; right column, top to bottom, 2,2 bis ( $\mathrm{p}$ chlorphenyl) 2 chloro 1,1,1 trichloroethane, DDE (2,2 bis (p-chlorphenyl) 1,1 dichloroethylene), and 2,2 bis (p-chlorphenyl) ethane. Note that iodo-DDT does not fit because the p-substituent is too large, while in DDE it is the change from tetrahedral bond angle that causes non-fit.

the number of vacant interspaces declines proportionally for all sizes as shown on the left in Fig. 6. Large anesthetic molecules, because they can enter only large sized interspaces, may be expected not only to decrease the number of vacant interspaces but to shift the distribution maximum to lower absolute values as shown on the right in Fig. 6.

\section{The Enzyme Dehydrohalogenating DDT}

A recent report on the presence of an enzyme in flies that are resistant to DDT (Sternburg et al., 1954) together with an examination of the substrate 

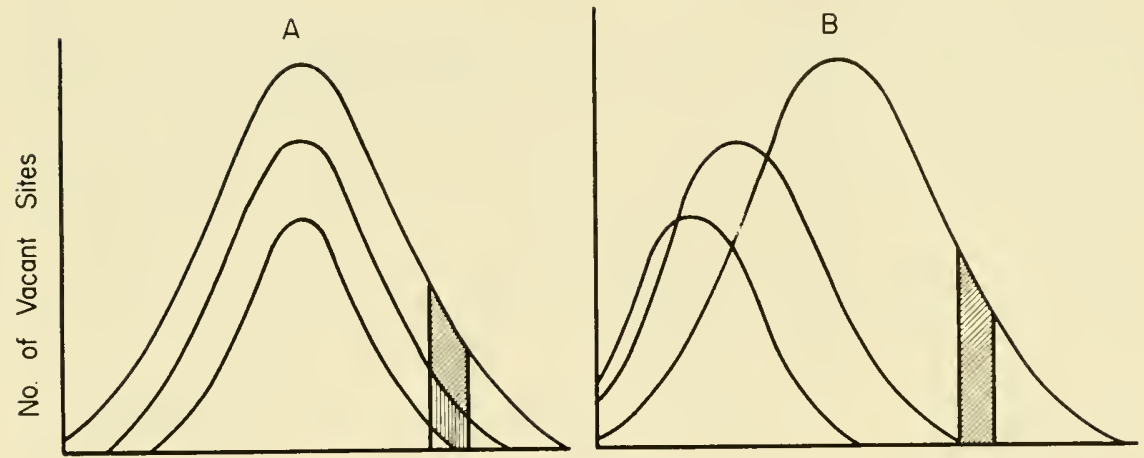

\section{Diameter of Site}

FIG. 6. The number of sites in a distribution is plotted against the size of a site in (A) top curve all sites vacant; middle curve, $25 \%$ of all sites occupied; lowest curve, $50 \%$ of all sites occupied. The vertical bar indicates DD'T sites. In (B), curves are as above, but the molecule filling the membrane occupies large sites only and hence shifts the mean interspace size as well as filling sites; DDT sites indicated as above.

specificity of this enzyme, makes it possible to compare substrate with receptor specificity. The reaction of DDT with alkali represents a dehydrohalogenation that may be written as below.
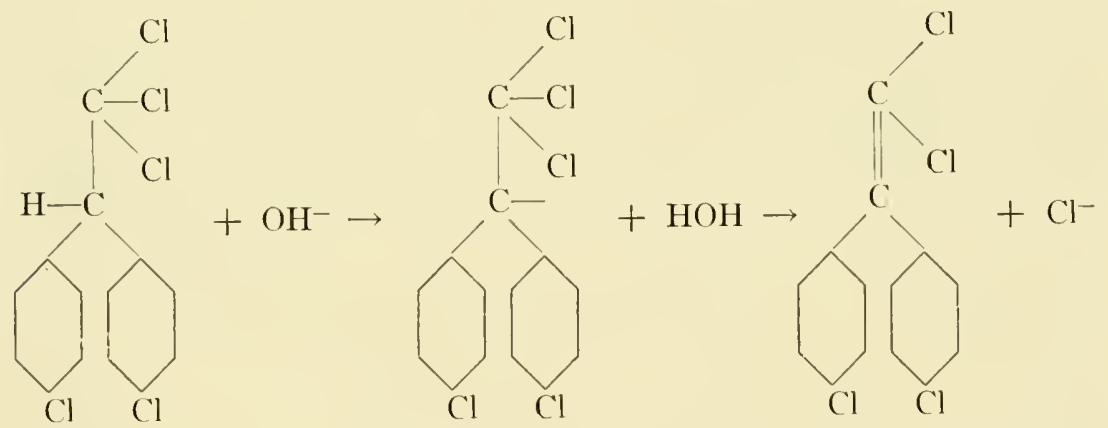

The hydrogen on the 2 carbon of ethane is very dependent for its reactivity on the p-substituent of the benzene rings; $\mathrm{CH}_{3} \mathrm{O}$ - makes the hydrogen least, and iodo- the most reactive. Since DDT, in resistant flies, is transformed by enzymatic action into the non-toxic dichlorethylene derivative (DDE), it is reasonable to expect that a dehydrohalogenation, similar to that taking place with alkali, has occurred. The relationship between p- substituents and enzymatic reactivity is completely at variance with this reaction mechanism as the data in Fig. 7 show. Aside from DDT itself, the most reactive compound is p- $\mathrm{CH}_{3} \mathrm{O}$ - which ought to be the least, and one of the least reactive is iodowhich should be one of the best. It is also clear that the enzyme shows precisely 


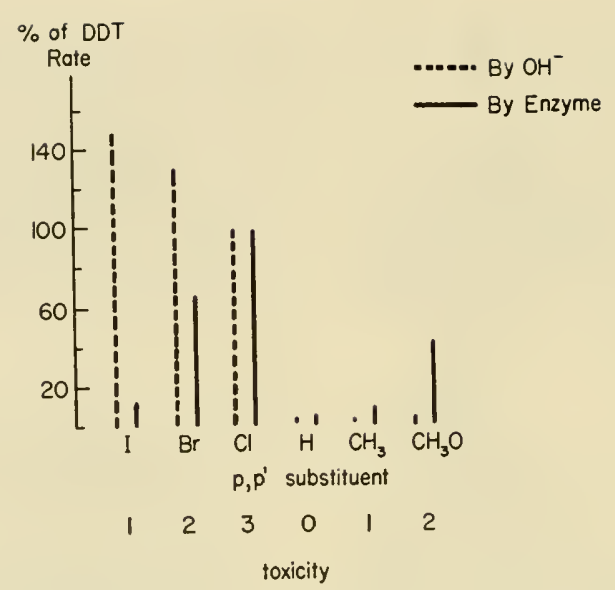

FIG. 7. The rate of transformation of DDT to DDE by alkali and by enzyme is compared with rates for homologs of DDT differing only in their p-substituents. An indication of the approximate toxicity of the substances to insects is given at the bottom of the diagram (Sternberg et al. 1954).

the type of substrate specificity as that of the receptor, e.g. the most toxic compounds are the best substrates. While DDT shows an action superficially similar to the application of acetylcholine to sensitive structures in mammals, it is not an anticholinesterase (Hartley and Brown, 1955).

One difficulty in this problem is the lack of any general understanding of the mechanism of enzyme action; it is not intended here to do more than suggest a mechanism applicable to this particular reaction. Attack on the 2-hydrogen of DDT seems unlikely for the reasons mentioned previously. 'The same end product, DDE, could be obtained by a removal of one of the $\mathrm{Cl}$ atoms on the 1position of DD'T; the hydrogen would then undergo a spontaneous release and DDE would result. This reaction sequence would permit enzyme activity to be independent of the p- substituent which is not precisely the case. It may be argued that the nature of the p- substituent determines the extent of binding of substrate to enzyme; this would explain the low reactivity when $\mathrm{H}$ is the $\mathrm{p}$ substituent but would fail to explain the poor reactivity of the p-iodo compound. A comparison between Figs. 5 and 7 shows that enzyme action can be correlated with "goodness of fit" or with the same parameters that were developed to explain the convulsant activity of DDT.

In estimating the size of an interspace into which DD'T would fit, the radius of curvature of the cylindrical macromolecules was set by the points of tangency of the two $\mathrm{p}-\mathrm{Cl}$ atoms to the surface of the macromolecule. An identical radius of curvature is defined by the $\mathrm{p}-\mathrm{Cl}$ atom of one ring and an ethane- $\mathrm{Cl}$ on the same side of the molecule. The cylinders so specified have a radius of $17.5 \AA$ 
and are separated from each other by $2.0 \AA$., a figure that might be considered set by the roughness of the surface of the macromolecule, or its deviation from a circular shape. A circle of $3.8 \AA$. radius can be inscribed in the interspace specified above and it seems likely that it was derived from an interspace considerably larger, perhaps $4.8 \AA$. This is so because of the great precision with which DDT must be oriented with respect to the interspace, a fact that makes larger interspaces much more probable sites for DD'T. Very large interspaces will, however, allow the penetration of DDT in other orientations and thus result in the filling of the membrane with molecules that do not give a good fit with the interspace.

If peripheral nervous structures upon which DD' $\mathrm{T}$ acts have the same relative permeabilities to $\mathrm{Cl}^{-}, \mathrm{K}^{+}$, and $\mathrm{Na}^{+}$as squid axons, then the mean interspace size shown previously ( $4.2 \AA$.) may be expected to represent the mean size for a DDT-sensitive membrane. It has been suggested that the DDT molecule, to produce its effect, must occupy interspaces that are large compared with the mean size (perhaps $4.8 \AA$.). Since the effects produced by DDT depend upon there being relatively few DDT-occupied channels and since all interspaces are capable of oscillating through the entire range of possible sizes, selectivity with respect to occupying only a small number of channels is obtained if the molecular size is large compared with the mean. Selectivity is demanded by the model because if, per unit of time, $20 \%$ of the interspaces can be occupied by DD'T in one case, and $0.2 \%$ in the other, a large fraction of the membrane interspaces will be occupied with a small number of DDT molecules per chammel. In the second case, the cliange in interspace size distribution occasioned by the introduction of DDT into a few large interspaces suffices to insure the filling of already occupied channels. If DDT encounters a membrane of a mean size somewhat larger than itself, a somewhat different reaction may take place. From the discussion of narcosis it would appear that substances that are smaller than the mean interspace size are good blocking agents. DDT is, however, so very insoluble that its appearance in the membrane in concentrations necessary for narcosis might be expected to occur only in times far longer than those of the experiment; as a first approximation the substance would appear inert. Those DDT molecules reaching the membrane may be expected to bind well to interspaces of appropriate size but a single molecule is doubtless insufficient to prevent membrane molecules from undergoing thermal motion such that the DDT becomes repeatedly bound and unbound. The $\mathrm{C}-\mathrm{Cl}$ bond of the ethane part of the molecule is considerably weater than that of the $\mathrm{p}$-Cl binding so that if the repeated straining of the molecule results in some chemical reaction this may be expected to be the removal of an ethane $\mathrm{Cl}$ atom. The subsequent decomposition of the free radical would yield DDE, and the substrate specificity of this reaction may be expected to conform with the results shown earlier. 


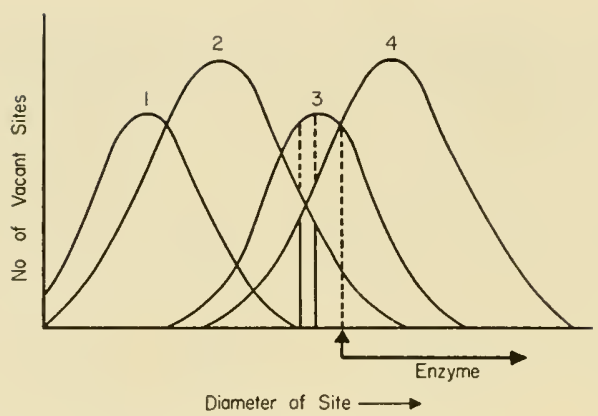

FIG. 8. The suggested interspace site distribution for DI)T-sensitive organisms is shown as curve 2, while curve 4 represents the distribution for resistant organisms. The addition of a synergist changes curve 2 to 1 , and curve $t$ to 3 . DDT sites are shown by the vertical bar, and enzyme sites by the area to the right of the dotted vertical line.

The action of certain non-insecticidal homologs of DDT is helpful in analyzing the relationship between enzyme and receptor. Thus, 2,2 bis (p-chlorphenyl) 2 hydroxy ethane synergizes the action of DDT on resistant flies only, and is an inhibitor of the enzyme (Moorefield and Kearns, 1955). Such an action is predictable if the substance is an anesthetic that, because it occupies more than just the free volume in an interspace, shifts the interspace mean size to smaller values, as shown in Fig. 8, as well as reducing the number of sites that are too large for DD'T (enzyme sites). Substances that have no substituent of high electron density and are small enough to penetrate in many. different orientations like diphenylhydroxymethane neither synergize the effects of DDT nor inhibit the enzyme at the concentrations studied, while substances like bis (p-chlorphenyl) hydroxymethane do apparently have enough binding strength to inhibit the enzyme but do not occupy much more than the free volume of an interspace and therefore do not change the interspace distribution. Because all the inhibitors are about $10^{3}$ more water soluble than DDT it is impossible to estimate relative membrane concentrations but even in their highest concentrations, inhibitors inactivate only $70 \%$ of the DDT-ase.

The preceding discussion has suggested that the process of obtaining organisms resistant to DDT selects from a population those individuals with a different membrane structure. The conventional notion that the presence of an enzyme detoxifying DDT is responsible for DDT resistance is to be contrasted with the view that the enzyme is a consequence of a membrane structure that is not sensitive to DDT. It is extremely difficult to distinguish, experimentally, between these possibilities because they amount to saying that the receptor is somewhat more selective in its stereochemical requirements 
than is the enzyme. It is also apparent that resistance to DDT can be obtained in other ways: the interspaces may be too small for a fit, or local alterations in the surfaces of membrane molecules may result in changes in the surface roughness that prevent penetration.

\section{The Enzyme Hydrolyzing Acetylcholine}

Certain of the features developed in analyzing the relationship between DDT and DDT-ase suggest that a comparison with acetylcholine (ACh) and its enzyme ACh-ase might contribute further information of value in the analysis of membrane structure. At the outset it must be emphasized that owing to flexibility of the ACh molecule, and to certain peculiar relationships between inhibitors of either the receptor or the enzyme, information on the nature of the $\mathrm{ACh}$ system must of neressity be much more tentative than that for DDT. Because of the high order of complexity that is involved, experimental data are almost invariably subject to more than one interpretation; this fact puts a premium on explanations that can interrelate observations involving a wide variety of chemical or physical treatments.

The principal means of distinguishing receptor from enzyme in the $\mathrm{ACh}$ system is the differential action of certain drugs and substrates. Thus there are drugs that block the activity of the esterase and enhance the activity of the receptor, presumably by allowing $\mathrm{ACh}$ to remain in contact with the receptor for longer periods of time, while on other tissues the same inhibitor blocks both receptor and enzyme action. Again, there are drugs that block receptor activity without depressing the activity of the enzyme. Substrates for the esterase also have a differential action on the receptor; thus dimethylaminoethyl acetate is a good substrate and a poor stimulator of the receptor, while the reverse is true for butyrylcholine. Perhaps the first question to be asked is whether there is a role of physiological significance that can be assigned to ACh-ase at, for example, the neuromuscular junction (n-m-j) of twitch-producing muscles. While localization of the enzyme close to the cell membrane appears likely (Hellmann, 1952), we are left in doubt as to which of three general arrangements actually exists: (a) the enzyme is just outside the cell membrane, (b) it is just inside the cell, or (c) the membrane is a mosaic of areas of enzyme and receptor. Arrangement (a) would serve mainly to attenuate the material reaching the receptor, and would require larger signals from the pre-junctional fibers; it might be thought of as sharpening the chemical signal by reducing low concentrations of $\mathrm{ACh}$ to subthreshold values and being overwnelmed by larger concentrations. This arrangement requires ACh-ase to be present in low concentrations and seems therefore unlikely. Arrangement (b) would be presumed to prevent the back diffusion of ACh once it has passed through the membrane and presumably exerted its physiological effect; there 


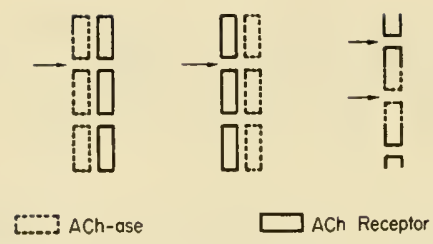

FIG. 9. Three arrangements of receptor relative to enzyme for the acetylcholine system are shown: on the left, the enzyme is just outside the receptor; center, enzyme is just inside; and right, areas of receptor and enzyme alternate over the membrane surface. Arrows indicate direction of diffusion of $\mathrm{ACh}$.

would hardly seem to be much functional significance in this arrangement because the concentrations of $\mathrm{ACh}$ are so small and the material is diffusing into an almost infinite volume of cytoplasm. In (c) the effect would be that of saying that a greater or lesser area of the membrane is inactive in the sense of being a receptor, since it is given over to enzyme action. Fig. 9 shows the three arrangements discussed.

A decision as to the extent of the coupling between enzyme and receptor can be based on studies of specific inhibitors of the enzyme. If the inhibitor is a substance that reacts solely with the enzyme, its introduction should result in the persistence of $\mathrm{ACh}$ for a longer period of time at the receptor, or the escape of ACh should now be diffusion limited. If receptor and enzyme are both consequences of membrane structure, a block of the enzyme may result in the production of more receptors as well as a persistence of $\mathrm{ACh}$ at the membrane; the experimental problem is then, to distinguish between persistence of $\mathrm{ACh}$ and an actual increase in the number of receptors. According to conventional explanations of the action of ACh-ase inhibitors several points are not clear. On peripheral nerve, inhibitors (such as DFP) depress conduction to the point of failure (Crescitelli et al, 1946) while at the $n-m-j$ the first action of inhibitors is to facilitate excitation. In the arthropods where ACh cannot be demonstrated to exert a physiological action (Prosser, 1952), inhibitors excite. All of these excitable systems have ACh-ase activity and the apparent differences between them can be explained by assuming that nerve does not have $\mathrm{ACh}$ receptors (as it is excited by a $\mathrm{K}^{+}-\mathrm{Na}^{+}$mechanism), while arthropods have a transmitter that differs in some respects from ACh. The suggestion has been made several times, most recently by Zupancic (1953), that receptor and enzyme for the ACh system are in fact identical, but the information used to support such claims has not generally been able to dispose of the objection that certain substances are rather selectively reactive with either enzyme or receptor. The evidence to be presented here is not that receptor and enzyme are identical but that they are both aspects of the activity to be expected from a membrane based on previously discussed structural considerations. 
Since we are going to examine the consequences of having ACh-ase activity localized in some of the interspaces of the membrane, it is worthwhile to state just what these are. With respect to the inhibition of the enzyme, it must be possible to accomplish this without filling more than a fraction of the interspaces with inhibitor. Because the membrane is concerned with the transfer of materials associated with the operation of cellular metabolic processes, the total inhibition of such transport may be expected to be incompatible with function even over short intervals of time. The inhibitors that are spoken of as 'competitive' are, according to this interpretation, incorrectly so classified because their principal contribution to $\mathrm{ACh}$-ase inhibition is the decrease in mean interspace size that they bring about. This action effectively shuts off access of substrate to enzyme without the requirement that an inhibitor molecule must occupy every active site. Similarly, the phenomenon of substrate inhibition shown so strongly by ACh (Augustinsson and Nachmansohn, 1949) may be brought about in part by a mechanism similar to that suggested for inhibitors, rather than by the formation of a 'super complex'. Interestingly, ethyl chloroacetate which lacks the two points of attachment generally required for complex formation, shows substrate inhibition while the tertiary analog of $\mathrm{ACh}$, dimethylaminoethyl acetate which binds almost as well as $\mathrm{ACh}$ and has two points of attachment, does not (Berman et al, 1953), suggesting that both the quaternary structure and a $\mathrm{Cl}$ atom of chloroacetate distort the interspace distribution because of their non-conforming shapes, while the tertiary structure is a better fit.

The apparent concentration of enzyme in vitro and in vivo is of importance for the present discussion because it cannot be claimed that the greatest amount of ACh-ase can be extracted from tissues containing the largest amounts. Quite aside from the large losses caused by adsorption to cellular components, if, as has been suggested, enzyme activity depends upon some supramolecular organization, a premium is placed upon those methods of extraction that favor molecular aggregation of various specified sorts. In the membrane, enzyme activity may be related to the number of interspaces greater in size than a limit fixed by the size of $\mathrm{ACh}$ in a required orientation, but the binding of substrate will be greatest for those interspaces that correspond most closely to the size of $\mathrm{ACh}$ and so will the barrier to penetration of $\mathrm{ACh}$. Maximum rate of reaction will presumably be obtained with interspaces somewhat larger than those for optimal binding because of the diminished penetration barrier for such interspaces. For extracted enzyme, similar considerations may be expected to apply, if activity is taking place by virtue of some organization of macromolecules. For a cylindrical molecule of radius $20 \AA$. and length $100 \AA$. a molecular weight of about 100,000 can be calculated (assuming a density of 1.2). Since even purified ACh-ase is poorly characterized by present-day standards for proteins, it is possible that the reported molecular weight of $3,000,000$ 
represents an aggregate of 30 molecules or a dispersion of aggregates of different size. There is no certainty that the interspace distribution in micelles of membrane molecules in solution (ACh-ase) corresponds to interspace distributions in the cell, hence attempts have been made to assay ACh-ase in intact tissue (Schleyer, 1955). Penetration barriers to ACh have necessitated the use of other substrates, the latest of which is ethyl chloroacetate. The results of these studies are not entirely satisfactory as only $60 \%$ of the extractable ACh-ase is reached by the substrate - suggesting that perhaps the extracted micelles are a more open structure than the membrane. In addition the presence of another enzyme in large amounts was noted. This enzyme was not inhibited by ACh-ase inhibitors and the results might be explained if the substrate were decomposed by some $\mathrm{Cl}$-membrane- $\mathrm{C}=\mathrm{O}$ interaction in addition to the cationmembrane $\mathrm{C}=\mathrm{O}$ interaction.

The possibility exists that the actions of a variety of drugs that apparently selectively affect either receptor or enzyme, can be explained as a consequence of the change in mean interspace size that they induce. It has been noted by Friess and McCarville (1954) and by Bernhard (1955) that in eserine the center of positive charge and the carbonyl group are 1.1 to $1.4 \AA$ (depending upon model parameters) further apart than in $\mathrm{ACh}$ at full extension. This is a quite remarkable situation since the presumed effectiveness of eserine as an inhibitor of the enzyme might be expected to depend upon a precise correspondence between reactive groupings. The observation is understandable if it is assumed that sites fitting $\mathrm{ACh}$ are small when compared with the mean interspace size, while eserine interacts with sites of a larger size. Fig. 10 has been constructed to represent the relationship of $\mathrm{ACh}$ receptor to enzyme sites assuming that the action of an inhibitor is to shift the distribution of site sizes to lower mean values. The proper amount of enzyme inhibition may be expected to increase the number of receptor sites, while larger inhibitions, or larger doses of inhibitor will eliminate receptor sites. The change in the profile size of the molecule as $\mathrm{CH}_{2}$ groups are added to $\mathrm{ACh}$ is shown on the diagram; it is not clear whether or not the receptor is able to operate by a cation- $\mathrm{CH}_{2}$ group interaction, but the fact that enzyme action fails (hecause of the screening of $\mathrm{CH}_{2}$ groups) at butyryl choline, while the receptor is active, is understandable from the diagram. A functional role for ACh-ase is also apparent; it is concerned with the removal of $\mathrm{ACh}$ from those interspaces that are not of receptor size, and thus of preventing narcosis. Butyryl choline has less need of such an enzyme because of the smaller number of sites larger than this substance.

Evidence has been presented that ACh-ase activity depends upon the existence of anionic and esteratic sites (Wilson, 1951a), hence within the interspace we must assign such specific binding points. Unfortunately there are several possibilities in this regard and any decision between them must rest on the ability of such a model to correlate a variety of observations on both receptors 


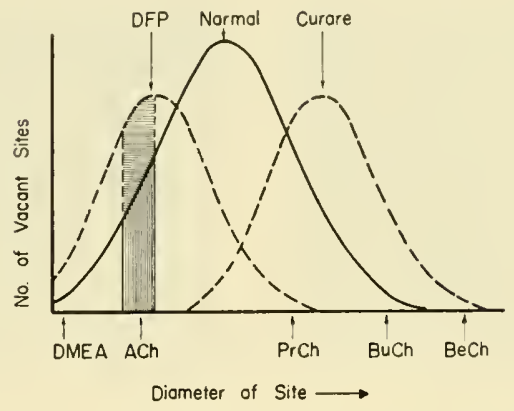

Fig. 10. The number of vacant interspaces is plotted against their size for a normal membrane (solid line), for a membrane where the mean interspace size has been decreased (DFP), and for a membrane where the mean interspace size has been increased (Curare). Receptor-sized interspaces for $\mathrm{ACh}$ are indicated by the vertical bar, enzyme sites by the area to the right of the bar. Along the abscissa is shown the relative profile size of analogs of $\mathrm{ACh}$, (dimethylaminoethyl acetate, and propionyl, butyryl, and benzoyl choline) in an orientation suitable for enzyme action. It is not suggested that receptor action for these analogs can be predicted from the curve, but that one must expect marked penetration barriers for higher homologs.

and enzyme. If, at the entrance to an interspace, a membrane molecule has esteratic (E) and anionic (A) sites in alternation every $60^{\circ}$, two possibilities are distinguishable: (a) the membrane molecules rotate freely about their long axis, and (b) the molecules are non-rotating. The first arrangement gives rise to sites: AAA, AAE, AEE, and EEE, while the latter gives only AAA and EEE sites as shown in Fig. 11. It is also possible that an unknown or inactive site $\mathrm{X}$ is present so that for non-rotating molecules the pattern is AEX. A variety of experimental observations suggest, however, that at $30^{\circ}$ intervals around a membrane molecule a pattern $\mathrm{AEA}$ is repeated, giving rise to the rather complex distribution shown in Fig. 11. This arrangement has the advantage that the distance between $\mathrm{A}$ and $\mathrm{E}$ centers is about that in $\mathrm{AC}$, when the macromolecule is $20 \AA$ in radius. The validity of this model may be tested by comparing the fit of models of various substances with the results from enzyme inhibition. With the simple quaternary ammonium ions, tetraethylammonium ion is bound somewhat more firmly than tetramethylammonium ion (TMA) although not as strongly as one might expect from the increase in dispersion forces (Bernhard, 1955). Such binding studies do not take into account any dispersion in site size, a factor that may be of considerable importance. Some information on how TMA ought to be oriented is obtainable from a study (Wilson, 1951b) where it was shown that ACh-ase blocked with TMA still permitted the rapid reaction of thiolacetic acid with water. A further suggestion regarding TMA orientation is the fact that this substance is stimulating in its action on nervous structures, an effect not to be expected if the ion interacted 


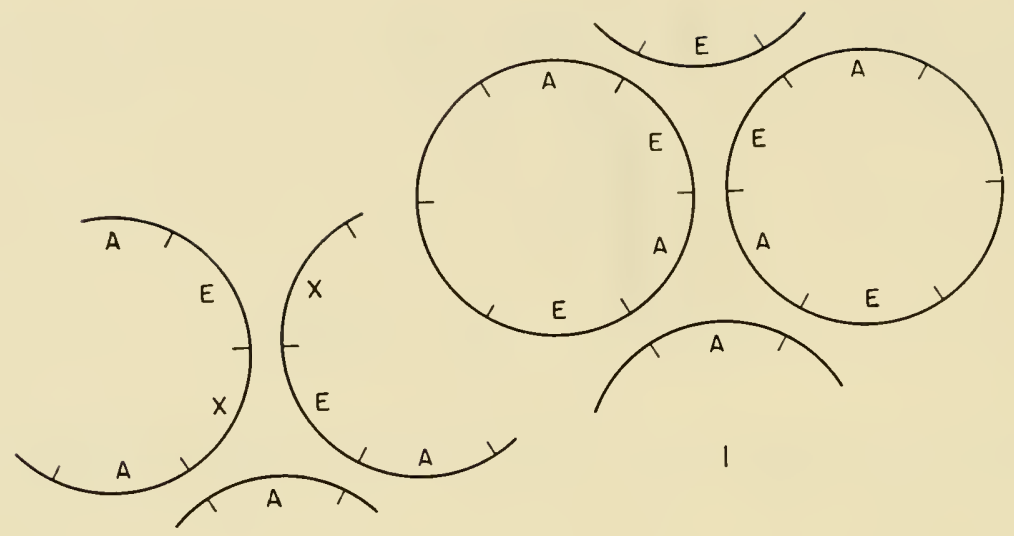

2

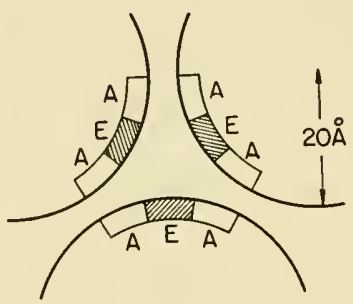

3

Fig. 11. Possible arrangements of anionic (A), and esteratic (E) sites as described in the text.

with only one of the membrane molecules. Thus, TMA should interact with two membrane molecules and enough space should be left in the interspace to accommodate thiolacetic acid; this is possible in the arrangement shown in Fig. 12. The greater asymmetry of TEA suggests that it will not approach any of the A sites as closely but that its binding by dispersion forces will be of importance. In the interspace $\mathrm{ACh}$ is shown in two orientations, one for enzyme and one for receptor action. Just which of these two arrangements will te favored depends upon the interspace size; in the diagram a small interspace ( $2 \AA$ separation) is shown, and hence the 'enzyme' orientation is not a good fit.

Certain features of the action of specific inhibitors of ACh-ase are helpful in testing the general validity of the arrangement of specific sites in the interspace. With TEPP, the anionic site is functional, while it is not with DFP (Wilson et al, 1955). Again, the action of eserine and neostigmine is contrasting in that the latter substance depolarizes. The fluorophosphate DFP also differs from eserine in that it can fit into an interspace of a size similar to those previously considered ( $2 \AA$. separation) while eserine and prostigmine require larger sized 

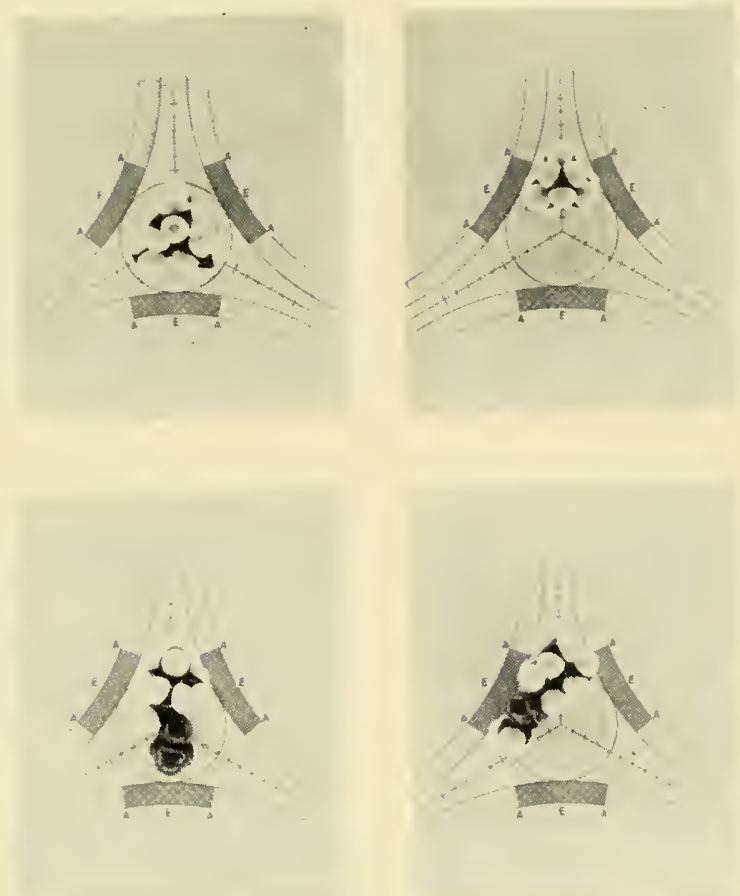

Fig. 12. The fit of various substances into interspaces formed by membrane molecules of radius $20 \AA$. and $2 \AA$. separation. Upper left, tetraethylammonium ion; upper right tetramethylammonium ion (free space around the lower A site is more than adequate to accommodate thiolacetic acid); lower left, ACh in a 'receptor' orientation; lower right, $\mathrm{ACh}$ in an 'enzyme' orientation.

interspaces. Fig. 13 shows these molecules fitted into interspaces of the appropriate size and indicates that the diethylphosphoryl enzyme derived from TEPP has indeed a free anionic site on each side of the interspace while the DFP enzyme has not. In addition, eserine and neostigmine bind differently in the interspace.

\section{The Receptor for Acetylcholine}

The discussion of the ACh receptor will be confined to that at the n-m-j of twitch-producing muscles because the nature and the speed of the processes presumed to be taking place are more easily related to events accompanying excitations in axons. The excitatory action of $A(\mathrm{C} h$ at the junction is presumed to produce a generalized permeability increase to all ions (Fatt and Katz, 1951) in contrast with the data on axons that demands an increased $\mathrm{Na}^{+}$permeability. Because ACh appears to be small compared with an interspace size that will 

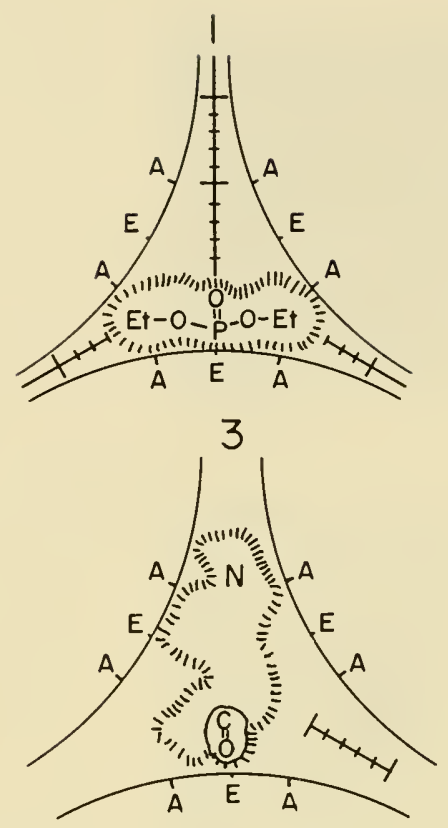

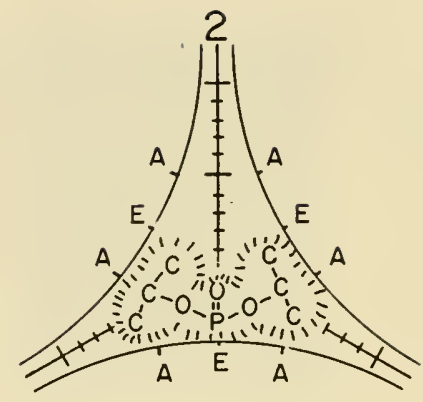

4

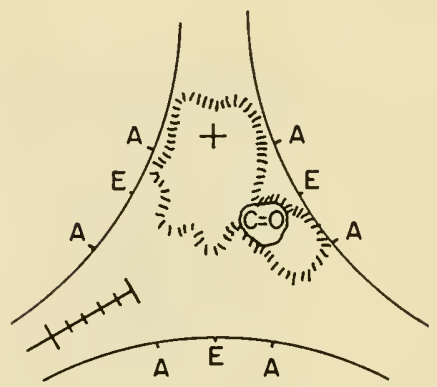

Fig. 13. (1) A 20-2 (radius $4 \AA$.) interspace occupied by diethylphosphoryl-; (2) the same interspace occupied by di-isopropylphosphoryl- (from DFP), the E sites are occluded; (3) an interspace of radius $5 \AA$ occupied by eserine; (4) the same interspace occupied by neostigmine.

accommodate ACh-ase inhibitors, and because inhibitors of the receptor require interspaces of even larger size, it is possible that the membrane at the $n-m-j$ has a much larger mean size than that of axons, or that the dispersion of interspace sizes is very much greater for this membrane. This latter situation appears to offer better possibilities of explaining the action of various substances, and can be examined in some detail by studies on the inhibition of the ACh receptor.

When a single molecule has two quaternary ammonium ions separated from each other by a $\mathrm{CH}_{2}$ chain of $n$ carbon atoms, the possible modes for binding, with respect to the model are: (a) across the interspace between any two pairs of A sites, (b) along the interspace channel, one pair of A sites near the surface and another at some definite distance down the channel, (c) between the anionic sites of two adjacent interspaces, and (d) one cationic nitrogen is not bound and the molecule occupies the interspace in a variety of orientations. Undoubtedly many of these possibilities are realized to some extent, and the task of specifying a particular orientation is made correspondingly difficult. To recognize the principal mode of binding of a particular substance, certain tests can 
be made; the blocking action of hexamethonium $\left(\mathrm{C}_{6}\right)$ should be enhanced if the molecule binds by orientation (a) and one Me - group of $\mathrm{N}$ is replaced by Et - since this change improves the fit at the anionic site; further Et - substitution will spoil the fit. For orientation (b), triethyl substitution would be expected to yield an improved fit, because TEA just fits the interspace; the chain length is too short for (c) to be considered, and as the presence of two quaternary groups is essential for activity, (d) appears unimportant. The results of experiment (Wien and Mason, 1953) are in agreement with orientation (a). The far more powerful receptor blocking action of $\mathrm{C}_{10}$ cautions against the conclusion that it orients in the post-junctional membrane in a manner similar to $\mathrm{C}_{6}$ in the ganglion. While it is true that $\mathrm{C}_{10}$ has 4 more $\mathrm{CH}_{2}$ groups to enhance its binding, a conclusive indication of a basic difference in action is the diminution in activity upon Et - substitution in the quaternary nitrogens, and the marked species variability in sensitivity to $C_{10}$, suggesting a higher order of specificity than that of many other blocking compounds. Hence (a) is ruled out; orientation (b) seems unlikely because activity should be increased by ethylation of the nitrogens; while (c) is indicated both because the chain length gives a good fit, and because the result of binding two membrane molecules together, as shown in Fig. 14 might be expected to be a depolarization resulting from a disturbance of the normal packing. The selective action of $\mathrm{C}_{10}$ is understandable because its insertion depends upon very large distances between membrane molecules, and while chains that are too short cannot bind, chains that are too long will tend to restore some of the disorder that is created.

Curare and $\mathrm{ACh}$ are stated to compete for the same receptor; while this is a summary of the results of experiment, it seems scarcely credible that such a large and bulky molecule as curare $(+)$ tubocurarine, has a molecular topography even remotely similar to that of $\mathrm{ACh}$. True, curare has 2 quaternary $\mathrm{N}$ atoms at a distance very roughly two times the extended length of ACh but they are on opposite sides of the molecule, a feature that makes any dual interaction of this molecule with a surface rather unlikely. Nor, it is clear, are two quaternary nitrogens necessary for selective receptor blocking; $\beta$-erythroidine with but a single tertiary nitrogen is an effective agent. Loewe and Harvey (1952) have carefully examined the interquaternary distance concept and found little in the way of support for it. From our model it is clear that curare cannot orient in the interspace in any way other than with its long axis parallel to that of the membrane molecules. While the distance between charges in + -tubocurarine is $12.5 \AA$., their separation from each other as measured along the axis of the molecule is $10 . \AA$, precisely the separation in $\mathrm{C}_{6}$. The profile and the orientation of these molecules is shown in Fig. 14, together with that of $\beta$-erythroidine. This latter substance is helpful in this analysis because it illustrates that the peculiar shape of the molecule, one lighly complementary to that of the membrane molecules, can be a factor of importance in bringing 


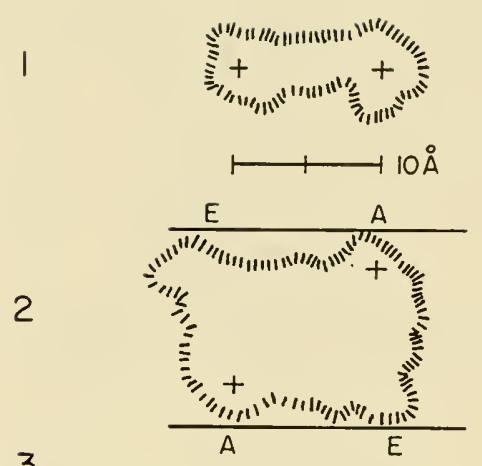

3

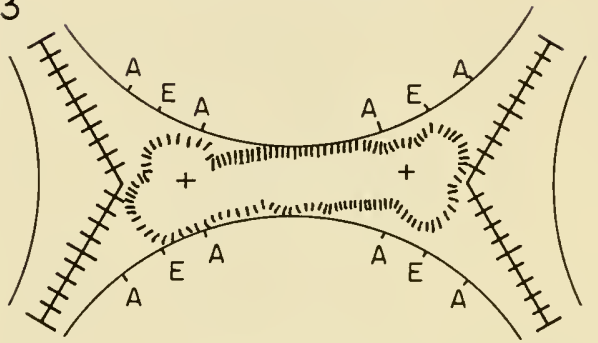

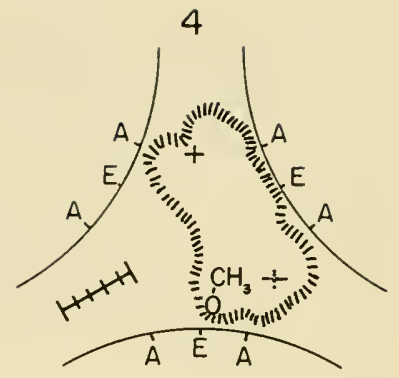

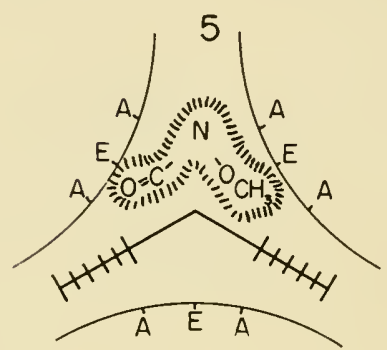

FIG. 14. (1) Hexamethonium at full extension (the trimethylammonium groupings are viewed from slightly different angles to show the variation possible); $(2)(+)$ tubocurarine viewed so as to show its largest profile, the parallel lines above and below the molecule represent the walls of an interspace, with $A$ and E sites shown; (4) shows tubocurarine in a view at right angles to that shown in (2), the dotted plus charge shown in the lower right is $10 \AA$ further lown the interspace than that charge shown at the top. The methoxy-grouping shown is in the same plane as the upper positive charge. The interspace radius is $6 \AA$.; (5) $\beta$-erythroidine in the same interspace as (4); (3) the extension of decamethonium between two membrane interspaces.

about receptor block, although there may be some contribution of the ether oxygens and the carbonyl group to binding at the E sites. The quaternization of this compound, by changing the $\mathrm{N}$-bond angles, destroys the special shape of the molecule and results in poor blocking agents. All of the powerful blocking agents require large sized interspaces for fit (a size of about $\left(\mathrm{Mg}^{++}\right)_{2}$ or $6 \AA$.) and have structural features that will cause a condensation in size of such interspaces, or a shift of the interspace distribution to larger mean values. This, as has been shown in Fig. 10, leads to a reduction in the number of interspaces into which $\mathrm{ACh}$ will fit, with little or no change in the number of 'enzyme' interspaces.

Two ions, $\mathrm{Mg}^{++}$and $\mathrm{Ca}^{++}$, exhibit a special case of antagonism at the $\mathrm{n}-\mathrm{m}-\mathrm{j}$ and it seems that while many types of reactions may be involved, the principal steady state situation is conditioned by the tendency of $\mathrm{Ca}^{++}$to occupy inter- 


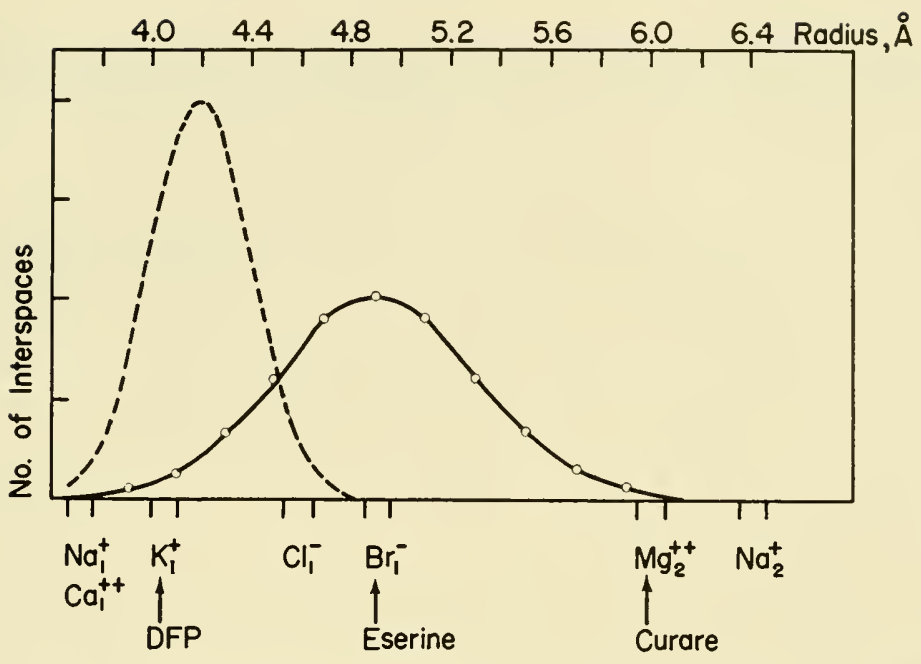

Fig. 15. The interspace size distribution for the complex $n-m-j$ membrane (solid line) is compared with that for nerve (dotted line). The distribution is based on a similarity of action of $\mathrm{Mg}^{++}$and curare (and their similar size), $\mathrm{Mg}^{++}{ }_{-} \mathrm{Ca}^{++}$antagonism, and on the need for assuming that the membrane becomes permeable to all ions (doubly hydrated) during activity.

spaces at the low end of the size distribution, and to produce, thereby, more ACh sites, while $\mathrm{Mg}^{++}$at the high end of the distribution does precisely the opposite, as shown in Fig. 15.

There is difficulty in specifying the sequence of events concerned in junctional transmission; this stems mainly from an uncertainty as to whether a particular experimental treatment affects events leading to the release of $\mathrm{ACh}$ from the pre-junctional terminals, or affects the reactivity of the post-junctional membrane. The usual means of distinguishing between these processes, a comparison between the response to nerve stimulation and topically applied $\mathrm{ACh}$, appears in several respects to be unsatisfactory. Fig. 15 has been drawn without any attempt to separate actions on the pre- or post-junctional membranes. A recent study (del Castillo and Katz, 1955) in which the rate of delivery of ACh to a single junction has been controlled with great precision, illustrates part of the difficulty. When ACh was applied by electrophoresis to junctions of the frog sartorius, the maximal rate of rise of a transient depolarization was only a small fraction of the rate of rise of a normal e.p.p. We lack the means for duplicating the rapid changes in concentration of $\mathrm{ACh}$ with respect to time that are of physiological interest. The slow depolarizations obtained with externally applied ACh mean, according to the model, that the membrane is redistributing its structure at a rate that is not greatly inferior to the rate at which $\mathrm{ACh}$ is 
being introduced into the membrane. How difficult it is, therefore, to conclude that because a drug modifies junctional transmission and yet the response to topical ACh is unaltered, the drug is acting on the release of ACh. The great dispersion in membrane interspace size that appears necessary to account for the action of drugs and ions should also result in a greater instability than that found in the axon; it would not be unreasonable to find small leaks developing spontaneously. Whether these are pre-junctional and involve quantal $\mathrm{ACh}$ release, as suggested by Fatt and $\mathrm{Katz}$, or whether they are post-junctional and limited by membrane 'viscosity' effects seems not at all clear. As suggested previously, curare may be supposed to stabilize the post-junctional membrane by reducing the number of $\mathrm{ACh}$ receptors; it may also stabilize against thermally induced ion leaks. Charge is transferred across the post-junctional membrane considerably more rapidly than is the case for the axon; this may reflect a thinner membrane but also suggests that ions such as sodium with two shells of hydration may be of importance in effecting this rapid charge transfer. Thus, it is suggested, the release of $\mathrm{ACh}$ results in a small number of molecules filling 'receptor' sized interspaces, the distortion surrounding such an interspace results in a large number of $\left(\mathrm{Na}^{+}\right)_{2}$ size and larger interspaces, and the $\mathrm{ACh}$ is removed by hydrolysis or diffusion.

Variations in the physiological response to $\mathrm{ACh}$ are understandable from the model if the mean interspace size and the membrane viscosity are considered as variables from tissue to tissue. It is then possible for $\mathrm{ACh}$ to inhibit function by hyperpolarization of the membrane, and for it to have its excitatory action integrated over periods of many minutes. Certain generalizations with respect to inhibition and synergism are worth commenting upon. If an excitatory substance requires sites that are small as compared with the mean of a distribution, the inhibition of such excitatory action directly (e.g. by occupying the sites that are to be acted on by the excitant with another molecule) is impossible unless substantially all of the sites in the membrane are occupied with inhibitor. Indirect inhibition, (e.g. by shifting the distribution of interspace sizes so as to diminish the number with small size) by condensing the size of a few, large sized interspaces, can be brought about without cccupying very many interspaces. Specific inhibition, (e.g. the inhibition of one junctional transmission system without influencing others) can be effected if the dispersion in interspace sizes is different for two systems, because a molecule that is very large, compared with the dispersion on which it acts as an inhibitor, may be equal to the mean size for the second dispersion, where it will be relatively inactive. Molecules of a size such that they may be taken up by half of all the interspaces in the membrane may be expected to decrease the mean interspace size. Such a change may be expected to give variations in the number of interspaces that can act as receptors for chemical transmitters or for $\mathrm{K}^{+}$. Large decreases in 
mean interspace size must be expected to make impossible the transformation of the membrane into an ion permeable structure.

\section{References}

Augustinsson, K. B. and 1). Nachmansohn. 1949. Distinction between acetylcholineesterase and other choline ester-splitting enzymes. Science 110: 98.

Berman, R., I. B. Wilson and D. Nachmansohn. 1953. Choline acetylase specificity in relation to biological function. Biochim. et Biophys. Acta 12: 315-324.

Bernhard, S. A. 1955. J. Am. Chem. Soc. 77: 1966-1971.

Brink, F. 1951. in Nerve Impulse, Macy Foundation, New York.

Crescitelli, F., G. B. Koelle and A. Gilman. 1946. Transmission of impulses in peripheral nerves treated with di-isopropyl fluorphosphate (DFP). J. Neurophysiol. 9: $2+1-252$.

Curtis, H. J. and K. S. Cole. 1938. Transverse electric impedance of the squid giant axon. J. Gen. Physiol. 21: 757-765.

Danielli, J. F. 1941. On the permeability change of stimulated nerve. J. Physiol. 100: $117-124$.

Davson, H. and J. F. Danielli. 1943. The Permeability of Natural Membranes. Macmillan Co. New York. 361 p.

Del Castillo, J. and B. Katz. 1955. Localization of acetylcholine receptors. J. Physiol. 128: $157-181$.

Fatt, P. and B. Katz. 1951. An analysis of the end-plate potential recorded with an intracellular electrode. J. Physiol. 115: 320-370.

Fatt, P. and B. Katz. 1952. Some problems of neuro-muscular transmission. Cold Spring Harbor Symp. Quant. Biol. 17: 275-280.

Ferguson, J. 1951. Relations between thermodynamic indices of narcotic potency and the molecular structure of narcotics. In "Mecanisme de la Narcose". Coll. Internat. Centre Nat. Rech. Sci. 26: 25-39.

Friess, S. L. and W. J. McCarville. 1954. Nature of the acetylcholinesterase surface. I. Some potent competitive inhibitors of the enzyme. J. Am. Chen. Soc. 76 : $1363-1367$.

Grundfest, H. 1955. The nature of the electrochemical potentials of bioelectric tissues. In "Electrochemistry in Biology and Medicine" ed. T. Shedlorsky. pp. 141-166. New York. John Wiley and Sons. 369 p.

Häfliger, E. 1948. Der Einfluss der Temperatur auf die Giftwirkung des DDT bei Honigbienen (Apis mellifica L.). Exper. 4: 223-225.

Hartley, J. B. and A. W. A. Brown. 1955. The effects of certain insecticides on the cholinesterase of the American cockroach. J. Econ. Entomol. 48: 265-269.

Hellmann, K. 1952. Cholinesterase of motor end-plate region. J. Cell. \& Comp. Physiol. 40: 421-426.

von Hevesy, G., E. Hofer and A. Krogh. 1935. The permeability of the skin of frogs to water as determined by $\mathrm{D}_{2} \mathrm{O}$ and $\mathrm{H}_{2} \mathrm{O}$. Skand. Arch. Physiol. 72 : 199-214.

Hodgkin, A. L. and B. Katz. 1949. The effect of sodium ions on the electrical activity of the Giant Axon of the squid. J. Physiol. 108: 37-77.

Hodgkin, A. L. and R. D. Keynes. 1955. The potassium permeability of a giant nerve fibre. J. Physiol. 128: 61-88.

Jenerick, II. 1953. Muscle membrane potential, resistance, and external potassium chloride. J. Cell. \& Comp. Physiol. 42: 427-448. 
Kofoed-Johnsen, V. and H. Ussing. 1953. The contributions of diffusion and flow to the passage of $\mathrm{D}_{2} \mathrm{O}$ through living membranes. Acta Physiol. Scand. 20: 60-76.

Lindquist, A. W., A. H. Madden and H. O. Schroeder. 1945. Effect of temperatures on knockdown and kill of houseflies exposed to DDT. J. Econ. Entomol. 38: 261-26t.

Loewe, S. and S. C. Harvey. 1952. Equidistance concept and structure-activity relation of curarizing drugs. Arch. exp. Path. Pharmakol. 214: 214-226.

Moorefield, H. H. and C. W. Kearns. 1955. Mechanism of action of certain synergists for DD'T against resistant house flies. J. Econ. Entomol. 48: 403-406.

Mullins, L. J. 1954. Some physical mechanisms in narcosis. Chem. Revs. 54: 289-323.

Mullins, L. J. 1955a. Olfaction. Ann. N. Y. Acad. Sci. 62: 247-276.

Mullins, L. J. 1955b. Structure-toxicity in hexachlorocyclohexane isomers. Science 122: 118-119.

Munson, S. C., G. M. Padilla and M. L. Weissmann. 1954. Insect lipids and insecticidal action. J. Econ. Entomol. 47: 578-587.

Pauling, L. 1940. The Nature of the Chemical Bond and the Structure of Molecules and Crystals. 2nd Ed. Ithaca. Cornell Univ. Press. 450 p.

Potter, C. and E. M. Gillham. 1946. Effects of atmospheric environment, before and after treatment, on the toxicity to insects of contact poisons. I. Ann. Appl. Biol. 33: 142-159.

Prescott, D. M. and E. Zeuthen. 1953. Comparison of water diffusion and water filtration across cell surfaces. Acta Physiol. Scand. 28: 77-94.

Prosser, C. L. 1952. Comparative Physiology. Saunders, Philadelphia.

Schleyer, IV. L. 1955. Electrical activity in electric tissue II. Evaluation of esterase activity in intact electroplax. Biochim. et Biophys. Acta 16: 396-103.

Sternburg, J., C. WI. Kearns and H. Moorefield. 1954. J. Agr. Food Chem. 2: $1125-$ 1128.

Verwey, E. J. IV. 1942. The reciprocal effect of ions and solvent in aqueous electrolyte solutions. Rec. trav chim. 61: 127-142.

Vinson, E. B. and C. IV. Kearns. 1952. Temperature and the action of D1OT on the American roach. J. Econ. Entomol. 45: 484-496.

Welsh, J. H. and H. T. Gordon. 1947. The mode of action of certain insecticides on the arthropod nerve axon. J. Cell. \& Comp. Physiol. 30: 147-171.

Wien, R. and D. F. J. Mason. 1951. Some actions of hexamethonium and certain analogs. Brit. J. Pharmacol. 6: 611-629.

Wilson, I. B. 1951 a. Mechanism of enzymic hydrolysis. I. Role of the acidic group in the esteratic site of acetylcholinesterase. Biochim. et Biophys. Acta 7: $466-470$.

Wilson, 1. B. 1951b. Mechanism of hydrolysis. II. New evidence for an acylated enzyme as intermediate. Biochim. et Biophys. Acta 7:520-525.

Wilson, I. B., S. Ginsberg and E. K. Meislich. 1955. The reactivation of acetylcholinesterase inhibited by tetraethyl pyrophosphate and diisopropyl fluorophosphate. J. Am. Chem. Soc. 77 : $4286-4291$.

Zupancic, A. O. 1953. Mode of action of acetyl choline. Theory extended to hypothesis on mode of action of other biologically active substances. Acta Physiol. Scand. 29: $63-71$.

Dr. Grenell: There are a few questions I would like to ask about this. I think it is a very stimulating set of ideas. One question is whether or not one 
can always consider excitation and block equivalent to raising and lowering the threshold. This is something that puzzles me somewhat. I am not sure these things are always the same. I think one has to take into account threshold to what, and what threshold one is talking about, because I think one can get shifts in this level within a range that might be said not to actually change the threshold.

Another question has to do with the problem of blocking at the surface, which I think, you felt was always the case. I wonder whether that is really so because certainly one can get particular preparations where you have destroyed this situation to some degree and still get evidence of a similar type of effect on very specific cell particulates. I am not quite sure that one always has to or can stick at the surface even though it is very tempting to do this under these circumstances.

One other question was the statement you made, I think at the beginning, about a change occurring with the alcohols at about C-6. If I remember correctly, on peripheral nerve, wasn't there a change from hypo to hyper polarization below that, quite a distinct and sharp change, I think? I wonder how this would fit.

DR. Mullins: I do not think I can say very much about the thresholds going up or down. I have given you a false impression if I gave you the impression that this sort of treatment leads to anything but a further appreciation of the difficulty in deciding just exactly what is going on in such a complicated structure. I am looking at one little hole among the millions that are around; these are filled with all conceivable kinds of substances that are pushing and pulling and tugging and twisting all different ways and the idea that, except in the abstract, we can see what the whole picture is is certainly not a valid one. When you talk about, for example, the relationship between metabolism and excitability or function, you have to consider whether metabolism is supplying entities which are influencing the membrane directly by being retained in these pore structures. Substances that are retained will in general be only those that find a happy home in this type of structure.

About the difference between agents that block at the surface versus blocking processes inside the cell, I think that there are an almost endless number of susceptible internal structures in the nerve cell and other cells in general; I think that to some extent this is what Gilbert Ling has in mind in proposing that there is an internal structural network which binds ions.

As to alcohols and the question of hyperpolarization or depolarization, this is a very complicated story because most narcotics are hyperpolarizing agents and it is very easy to show that you get a certain amount of increase in the membrane potential when such substances are applied, but lower alcohols (especially methanol) are strong depolarizing agents simply because they take enormously high concentrations of this substance to get the right activity to fill up the membrane spaces so that in the process of this you get quite a 
good bit of soap effect, as I would call it, which just disintegrates or disturbs the membrane in some way. I would prefer not to start with these lower alcohols which have confusing kinds of effects.

Dr. Grenell: If you apply such a polarization to repolarize an already depolarized structure, would this bring something out into the membrane from inside and consequently this is why the function has been restored to the situation? If you do not other than just repolarize it?

DR. MULLINS: I would think you would take something away rather than bring something out, but that is only a guess, I have no thought about it.

Dr. Robert Burton (National Institutes of Health): I have a couple of questions, one for Dr. Mullins and one for Dr. Folch-Pi.

The one for Dr. Mullins is whether or not any of the inositols produce a similar effect to the hexachlorocyclohexane derivatives, either stimulatory or narcotic, and whether or not they, perhaps, could inhibit the effect of these chlorine derivatives?

The question to Dr. Folch-Pi is whether or not the hexachlorocyclohexane derivatives inhibit the metabolism of inositol at all?

Dr. Mulbins: The suggestion was first made when the structure of the gamma isomer of hexachlorocychohexane was imperfectly understood. It was the same as, I think, what is called "meso" inositol and that therefore the possibility of competitive inhibition might exist. Indeed, it seems that you can demonstrate such an effect in bacteria between the S-isomer and meso-inositol; these are structurally equivalent (Barnes and Sogar, J. Gen. Microbiol. 10: $475,1954)$. But I do not believe that anyone seriously considers inositol to act in this manner for the simple reason that the substances are so enormously different in their general chenical constitution; one is a totally hydroxylated compound and the other is a totally chlorinated compound, and one would be much happier in the membrane than another, I am quite sure. Furthermore, it has never been possible to show that there is any even slight metabolic requirement for inositol, a nutritional requirement for inositol, or, therefore, any method whereby if you deprive insects, or even frogs, of inositol that they will become unhappy. So that this sort of an assumption seems to me illfounded.

Dr. Folch-Pr: I understand that cyclohexane has been used as an inositol anti-metaboloid. I think it was one of those wild ideas. I don't know what evidence there was for it and I think it was abandoned. On the other hand, I do think that there is evidence purporting to show that inositol is a vitaminlike substance of mammals. I think Wolley showed that it was necessary as a dietary factor in mice or rats. It is a good thing to think about.

On the other hand, I think I agree with Dr. Mullins; I wouldn't think that that would have much bearing on this particular example, i.e., on hexachlorocyclohexane. 


\section{Discussion}

Led by P. IT. Davies

Dr. Mullins' idea of a protein-column cell membrane is a most intriguing one, and it fits in particularly well with the helical models of protein molecules that have recently gained prominence. It is also encouraging that there are some who are taking the stereochemical or lock-and-key ideas of molecular interaction seriously enough to work out actual and very subtle correlations, which will be essential in the further development of membrane theory. However, before we abandon the lipid-film theories of membrane structure that have been with us for so long, starting back in the days of the Overton hypothesis and continued by such people as Danielli, it might be well to recall some of the evidence that remains in favor of a laminar structure for the cell membrane.

I might start off by recalling the evidence from physical chemistry about soap molecules, and I wish to say here merely that it has been shown by $x$-ray studies (McBain, 1950) that in concentrated soap solutions-sodium oleate, for example - the molecules tend to form bilaminar aggregates with the polar heads facing the aqueous medium and the non-polar tails inside. Thus they aggregate in such a way that their nonpolar tails are held together and are separated from the medium by their polar heads. The tails may intertwine in irregular fashion, but are held together strongly enough by van der Waals forces to overcome the attraction of the medium for the polar heads.

Secondly, there is the x-ray evidence of Schmitt, Bear and Palmer (1941) on myelin which indicates strongly the existence of a certain spacing. The myelin substance, which has water, neurokeratinogens and lipids in it, apparently goes into a laminar structure in which the polar groups of the lipid layers are in aqueous interfaces and attracted weakly to thin protein layers which lie between the lipid laminae.

A third, more recent, line of evidence in favor of the laminar structure comes from the work of Sjöstrand (1953) and others with the electron microscope. By his technique for working with ultra-thin sections, Sjöstrand has found that in nuclear membranes and mitochondria there is a tendency to find a three layered structure. In one case he found, in frozen-dried ultra-thin sections, two outer layers $160 \AA$ thick and an inner layer between them $210 \AA$ thick. Sjöstrand further finds that if he fixes his materials with osmium, which is a good electron stain and also a good protein stain, that the two outer layers take the stain nicely, whereas the inner layer does not. The presumption, according to Sjöstrand, is that this central layer contains some lipidlike material. This is direct evidence for a laminar structure of certain kinds of living membrane. A similar structure for what may be the functional axon membrane has been found by Geren (1954) and by Gasser (1955). The axon membranes shown in 
the electron micrographs of Geren and of Gasser are of the order of $300 \AA$ thick or less.

A fourth point should be made regarding the high permeability of cell membranes to water (Lucké, Hartline and Ricca, 1939). It might be objected that a cell membrane containing lipid, in which water is only slightly soluble, could not have a high enough water permeability to account for the experimental results. The work of Rideal (1925) has shown, however, that condensed monolayers of long-chain fatty acids lower the rate of evaporation of water (into a moderately evacuated space) to a value not less than about half that for a clean surface. A continuous lipid film in the cell membrane would therefore present no great barrier to the passage of water.

The fifth point has to do with the permeability of ions at oil-water interfaces, for if the cell membrane has a continuous lipid phase, any ions traversing the membrane will have to cross into a somewhat hydrophobic medium and out again. J. T. Davies (1950) has studied the diffusion of salts across a water-nitrobenzene interface, obtaining experimental values of the rate at which various alkali halides cross the interface from water to oil. He found permeability constants for the whole salts which were in the range between $1.4 \times 10^{-10}$ and $6.2 \times$ $10^{-8} \mathrm{~cm} / \mathrm{sec}$. This indicates that the free-energy barrier between an aqueous phase and an oil phase is itself sufficient to account for permeabilities which would be low enough, in fact too low in this case, to agree with the permeability values for the passage of ions across a cell surface, such as the membrane of the giant axon of the squid. The values for squid (Hodgkins and Katz, 1949) range from $7.2 \times 10^{-8}$ to $1.8 \times 10^{-6} \mathrm{~cm} / \mathrm{sec}$. Thus it is conceivable that the resistance of the squid axon to the passage of ions is due merely to the presence of a freeenergy barrier which must be surmounted as they pass into a lipid phase. The existence of membrane potentials can also be accounted for on this basis, as due to differences in the height of the barrier (before any potential difference arose) for the various anions and cations involved. The cell membrane will have two such interfaces, of course, one on each side of the lipid layer, and the net membrane potential will be the difference between the two interfacial potentials, which will in general be unequal. Furthermore, if the height of the barrier for a given ion, such as sodium, is different at the two interfaces the system exhibits the phenomenon of active ion transport. Such a difference could be due to the presence of an interfacial chemical reaction involving the ion at one of the interfaces. The picture of cell membrane potentials as compounded of two interfacial potentials might also account for the existence of the two separable components of membrane potential observed in frog muscle. I am referring to Dr. Ling's A and B potentials (1952). Two distinguishable fractions in the resting potential of frog nerve have also been observed by Shanes and Brown (1942) and Lorente de Nó (1947). This would be possible if the free-energy barriers at one of the interfaces could be changed without affecting those at the other inter- 
face. The barrier concept of permeability, which is required for a membrane with phase boundaries, differs distinctly from that of diffusion through a continuous phase. It has been expounded by Danielli (1952) and more recently in much greater detail by Zwolinski, Eyring and Reese (1949) and Polissar (1954).

Thus it seems that the laminar cell membrane with a lipid phase is capable of exhibiting quite a few of the known properties of excitable membranes, and it merits serious consideration until positive evidence against it is found.

Lastly, I would like to ask Dr. Mullins whether he considers it possible that the cell membranes could consist of two sheets of his protein columns attached to a central lipid layer?

\section{References}

Davies, J. T. 1950. The mechanism of diffusion of ions across a phase boundary and through cell walls. J. Phys. Coll. Chem. 54, 185.

Davson, H. and J. F. Danielli. 1952. The Permeability of Natural Membranes. Cambridge University Press.

Gasser, H. S. 1955. J. Gen. Physiol. 38: 709.

Geren, B. B. 195t. The formation from the Schwann cell surface of myelin in the peripheral nerves of chick embryos. Exp. Cell Res. 7, 558.

Hodgkin, A. L. and B. Katz. 1949. The effect of sodium ions on the electrical activity of the giant axon of the squid. J. Physiol. 108, 37 .

Johnson, F. H., H. Eyring and M. J. Polissar. 195t. The Kinetic Basis of Molecular Biology. Chapt. 11. John Wiley \& Sons.

Ling, G. N. 1952. The Role of Phosphate in the Maintenance of the Resting Potential and Selective Ionic Accumulation in Frog Muscle Cells. Phosphorus Metabolism. Vol. II. Johns Hopkins Press.

Lorente de Nó, R. 1947. Studies from the Rockefeller Inst. for Mled. Res. 131, 11.

Lucké, B., H. K. Hartline and R. A. Ricca. 1939. Comparative permeability to water and to certain solutes of the egg cells of three marine invertebrates. J. Cell. Comp. Physiol. 17, 237.

McBain, J. W. 1950. Colloicl Science. Chapt. 17. D. C. Heath and Co.

Rideal, E. K. 1925. On the influence of thin surface films on the evaporation of water. J. Phys. Chem. 29, 1585.

Schmitt, F. O., R. S. Bear and K. J. Palmer. 1941. X-ray diffraction studies on the structure of the nerve myelin sheath. J. Cell. Comp. Physiol. 18, 31.

Shanes, A. M. and D. E. S. Brown. 1942. The effect of metabolic inhibitors on the resting potential of frog nerve. J. Cell. Comp. Physiol. 19, 1.

Sjöstrand, F. S. 1953. Electron microscopy of mitochondria and cytoplasmic double membranes. Nature 171,30 .

Zwolinski, B. J., H. Eyring and C. E. Reese. 1949. Diffusion and membrane permeability. J. Phys. Coll. Chem. 53, 1426.

Dr. Morales: I am inclined to agree with Dr. Mullins that his view is not seriously embarrassed by demonstrating that there is a similar qualitative effect of narcotic agents on the interior bodies of the cell. 
Also, I am not sure that it is seriously embarrassed by Dr. Davies' idea that the membrane may be more complicated than Dr. Mullins' sketch, because I think the gist of Dr. Mullins' model, really, is that there is a two-dimensional lattice that can be affected by inclusions, and I don't imagine he would cling too tenaciously to the fact that these are protein cylinders.

I do wonder if there is a discriminating experiment between whether the narcotic agents have their effect on the membrane or on metabolic entities inside. If one accepts his model in the simplest terms it seems to me one should get the same curve for threshold activity versus number of carbon atoms, whether the agent is delivered on one side of the membrane or the other. I would like to ask him if he feels this is technically a sensible experiment, if he feels it is technically feasible, say, by micro-injection?

Dr. Mullins: I would like to say something to Dr. Davies first. I don't disagree particularly with what he has said. The only objection I would have is that it does not get down to this level of explanation that is satisfying to me, namely, the type of molecular alteration which is to bring about this electrical disturbance that we regard as a physiological function of the cell. As I gathered it, he would have some kind of an interfacial change at one of the surfaces take place which would alter the structure in some unspecified way so that it would become permeable to ions. I proposed the type of structure that I did because it seemed to me a way of having this alteration occur from application of physical principles and with a minimum of accessory hypotheses or special mechanisms. I tried to point out as I went along that it did involve assumptions, you have to size the thing somehow or other, and you have to agree that if things fit closely and exert a strong van der Waals type of attraction that you have--this could give rise to phenomena analogous to crystallization or to a lattice distortion or crystal defect, and that in the process of creating this local order you pay for it by an increase in the disorder surrounding this region for many tens of molcules away and therefore many tens of interspaces away. This gives at least a plausible mechanism for how excitation can be both initiated and amplified because I think we all agree that we need an amplifier action.

Now, the cylinders are very long. The ones I proposed were $100 \AA$ simply because people have settled on this kind of figure. I believe there is also a $50 \AA$ membrane thickness school which I certainly do not disagree with. But along this length it would be inconceivable to me that it is a solid lipid all the way through, the surface of these cylinders may be primarily $\mathrm{CH}_{2}$ groups, with gaps in which the polar groupings of the underlying structure, protein namely, would project through and these araas would suffice to explain many of the kinds of properties that we presently assign to purely a protein part of the membrane.

Also, there are the ends of the cylinders which I expect are protein, the largest 
part of the surface of the membrane is macromolecular and molecules collide with them much more frequently than they enter the holes.

I don't know whether that answers the question you made, Dr. Morales, to Dr. Davies or not. But on the question of the critical experiment that will tell you whether a narcotic will affect function by affecting some intercellular enzymatic mechanism or something of that sort versus its affecting the membrane directly, I wish I could think of something that would be decisive. I don't think that injecting things into the interior is a very good way, because it is extraordinarily difficult to keep the things in there if they can go into the membrane. Since you want this condition to persist for a little while, it is that sort of story where you inject cyanide into the cells and find that it doesn't have any effect, but when you bathe them in it from the outside they all die. The cells are in water and the cyanide just injected inside comes right out.

Dr. Morales: May I ask you something about just that? If you inject molecules inside cells they would be trapper inside. Now, if they effect, say, mitochondria or something of this sort, then they ought to have this effect anyway, but if your view is correct, they shouldn't have any effect if you put them inside. This is what I was driving at. Technically it may be nonsense and you might not be able to do this, but I wanted to know if you thought it was, in principle, discriminating.

Dr. Mullins: I see; I misunderstood the point. I think it is something worth while. I think you would have to agree these agents probably do affect the underlying biochemical systems. It is more a question of which they affect first. That was the point I was making.

Dr. David E. Goldman (Naval Medical Research Institute): I think this question of evaporation is pertinent both to what Dr. Davies has been speaking about and what Dr. Mullms has said. I would like to discuss this question of evaporation in respect to what Dr. Mullins had to say about his model. I am in agreement with Dr. Mullins that this is a reasonable sort of model to make. However, I feel that before one goes about predicting how well any particular molecule will bind to such a model one must consider how much energy it takes to pull an aggregate of such molecules apart. Unless one does this I do not think one can say which will fit better; all one can say is that certain molecules might be expected not to bind because they are too big. If, however, they fit, the question of how well they fit must always be weighed against the question of how well they fit to themslves, that is, something which makes a good liquid structure will be unlikely to bind simply because it doesn't gain much by binding on to a new type site.

DR. Folch-PI: Ever since the time of Overton and Meyer, people have been talking about oil, and the point is that there is no oil in tissues. There is no phase in tissue except in the adipose tissues where the oil forms a big blob that you 
can see in the microscope, that gives you an oil phase. There is no such thing as an absolutely hydrophobic lipid. In fact, they are amazingly hydrophilic. If you take, for instance, brain lipids, you can make a suspension in water just by making enough water. Furthermore, just try to get water out of brain lipids, for there is nothing in brain lipids except the oils.

Another thing is the fact that the more we know about tissue chemistry, the more we come to realize that most proteins and most lipids-structural ones, I am talking about - are tied into lipo-proteins of different size, different properties and different constitution. What this means in terms of the intimates is that wherever you have a lipid, presumably hydrophobic area, you get very close by, a hydrophilic, presumably protein layer. Therefore, I don't see how you can escape the fact that you have to deal with a mosaic. Of course, you would have the orientation that Dr. Schmitt and his workers have shown in which you would have a lipo-protein which would present only its paraffinic chains or its protein framework as the case might be. I think that when you are dealing with a membrane it would be another proposition altogether.

Then, one thing I must protest vehemently against is the idea of considering the membrane a mechanical thing, as a thing that does not change. It seems to me that most permeability phenomena are products of many events which are going on inside and outside and those sequence of events meet at the area of an interface. I think it is perfectly normal to assume that the structure of the membrane is very likely to change from one physiological condition to the other. I wonder if anybody has any comment on that.

Dr. DAvies: When I used the word "oil" I meant lipid, an oriented lipid, which would, I think, meet your requirements. I have no objections to all kinds of things happening at a lipid-protein interface, which would be in line with your statement that there have to be changes going on in order to allow permeability changes to take place.

Dr. Burton: I wanted to ask for a little more speculation from Dr. Mullins on the property of his holes. He has been speaking so far about these agents sitting on top of the membrane and blocking the hole. I wonder if he would care to speculate a little more on the property of the holes themselves, that is, insofar as there are varying sized holes for varying sized molecules. Are the polar groups sticking into the holes from the protein different in different holes to account for a certain amount of specificity? In this respect I presume we are speaking of simple diffusion, and I wonder if he would care to speculate on the property of these holes and the function of active transport perhaps an enzyme on the inside surface of these holes transporting the material inside.

Cilairmax (iERari): It seems to me that temperature would be an enormously valuable tool in getting at an analysis of this since the effective size of the holes is, according to this theory, directly a function of temperature. Also the rate at 
which a molecule gets through this long column would be influenced by the thermal oscillations of the protein columns which every so often ought to clamp down on it (the frequency for the pulsing of the hole versus the linear movement of the molecule). It might lead to some interesting temperature predictions.

Dr. Mullins: In the case of Dr. Gerard's question about temperature effects, I agree that this is really a most useful method for further investigation. One would predict on this basis that if you raised the temperature too high and expanded this lattice structure, you weaken the inter-molecular attractive forces of the membrane and some point will be reached where at one locus or another this force will be so attenuated between the molecules that you get effectively a breakdown or depolarization at that point which will, of course, cause a propagated disturbance and so high temperatures would be excitatory. If you lower the temperature you must expect an inverse effect, a decrease in the mean hole size, but also the possibility exists that the same kind of a crystallization that was produced by chemical agents is now produced by the lowered temperature, so that at one site or another you get again a disturbance which will result in depolarization and, therefore, in a propagated conduction. This would require rather special conditions, but it does explain the fact that there are both cold and hot receptors, both of which give stimulation in response to temperature changes up or down from some fixed point.

I must say that we were somewhat guided in these thoughts by the notion that the very precise temperature control which the mammalian organism is subject to could very conveniently be arranged by having an equilibrium existing in a membrane between melted and frozen parts such that any change in the distribution of such sites would cause stimulation, in other words, a thermostat, if you like. It would be easy to think of other mechanisms for a thermostat if the temperature regulation were not absolute. That is to say, the temperature regulation is absolute sometimes to as good as a tenth of a degree centigrade, a very small change on an absolute scale, and yet it always is at this particular value. Mechanisms involving viscosity changes and so on in the cells which might be expected to be as sensitive, but not in an absolute way, would fit in with this notion that Dr. Gerard suggests about temperature effect.

Cilatrman Gerard: Before you leave that, you have not picked up the particularly critical part of this, as I see it; namely, at a lower temperature the average size of the hole should be decreased somewhat and you should get a markedly differential action on your larger versus your smaller molecules in changing the excitatory and the narcotizing effects. Is that correct? And, if so, is there any evidence to support it?

Dr. MUllins: I cannot say for the lower temperatures. It is a fact, however, 
that DDT largely loses its effectiveness as the temperature is raised which suggests that the expansion in the site size suffices to inhibit the phase change suggested as the mechanism of action of the DDT at $20^{\circ}$.

In answer to the question about the substances and whether they prefer to be in their own pure liquid or in the membrane, these solids that we have been talking about, such as the insecticides, all have to be melted in order to be used, and, of course, the parameter is the latent heat of vaporization per unit volume of the molecule that determines whether it would like to go into its own liquid or whether it would like to go into the membrane. This is a physical-chemical kind of a concept which is not too applicable to this situation, because if the membrane were a homogeneous molecular lipid layer such as Dr. Davies was suggesting then you have to do work to separate the molecules of the lipid so as to insert this particular molecule we are talking about. Whether it would get inserted or not would depend upon the amount of work that you would have to do to separate the molecules at this new phase that it is going into versus the amount of work that would have to be done to get it back into its own liquid assuming that it is outside. But a membrane pore already exists by virtue of the structure of the membrane and no work has to be done to introduce a molecule of the appropriate size, so that the situation is a little bit different.

Chatrman Gerard: I think we might move off in a somewhat different direction.

Dr. OrR Reynolds (Chief, Biological Sciences Division, ONR): I would like to ask two questions - one is whether Dr. Mullins has considered the rare gases, which seem to be narcotic directly, as related to molecular weight and pressure, and how they would fit into this in view of the fact that the molecular size presumably is smaller than the molecules presented here?

The other question is whether or not this experiment has been done? If sites of crystallization are formed and therefore the spaces around them become larger, would you expect the larger molecules to become anesthetic before crystallization?

DR. LING: The point I want to raise is this: some time ago we proposed a hypothesis which has been called the fixed charge hypothesis and one aspect of that concerns permeability.

The fixed charge hypothesis deals mostly with charged particles or ions, and I think since it is probably generally agreed that ions play an important part in nerve excitation and electrical potentials, this might be an appropriate occasion to discuss it a little, and in relation to Dr. Mullins' presentation.

I think in Dr. Mullins' picture one striking feature was the presence of holes. If one goes back to the time when earlier theories were proposed for the mechanism of differential permeability and, for that matter, ionic accumulation and generation of electrical potential, one always could not escape the repeated observation and postulation that there were such holes. For instance, in one 
particular instance, that of the theory proposed by Boyles and Conway, the actual size of the hole was supposed to be such that it determined which ion was able to go through. Now, does this sieve idea apply here to the model that Dr. Mullins has presented? I think this is where I would like to bring in our fixed charge hypothesis.

If the different-sized, hydrated ions go into the cells through these channels or pores and the permeability depends upon how large they are and how large the sizes of these holes are, then a large size, charged particle like $\mathrm{Na}-\mathrm{I}$ mean hydrated charge particle-would certainly have a tendency to block the holes more than a smaller one. So if you study the rate of entry of one charged particle, let's say, $\mathrm{K}^{42}$, then the rate of entry of $\mathrm{K}^{42}$ should be blocked more by the larger sodium than, say, by a nonhydrated potassium. This is on the supposition that the number of holes are limited and that they are the rate limiting factor in the rate of entry of ions.

On the other hand, if you draw out the hypothetical charged size on one side of these triangular holes, then this applies almost equally well as a model for our thesis. The idea is to say that these proteins are highty charged and you can get some average value of the charge density by taking into account the total quantity of charged particles in the total amount of protein in the cell. I think Dr. Morales and Dr. Botts have calculated such an average distance between each of the charged particles if they are uniformly distributed and we had also made similar calculations. I think probably they would agree that something like 20 angstrom units would be the proper calculation. If you take that into account, then you arrive at a very interesting conclusion, because, if the surface is like the interior, filled with charged particles, then an externally placed ion will be subjected not only to a trans-membrane electrical field strength but it would also be subjected to the electrical field strength due to these ionized and charged particles. Now, their close proximity to each other makes it so that the electrical field strength, due to these charged particles, is extremely strong, so that an ion that would otherwise have entered the inside phase cannot do so directly but has to be attracted to these charges and then go in by disassociation.

What I am saying now is that the charged particles cannot just go in blindly depending on their size, but their entrance has to be preceded by a phase of association with the fixed charges on the surface followed by disassociation. This is, of course, based on the simple model which is in agreement with Dr. Mullins' model except that I added these charges.

A simple conclusion can be drawn from this because, if the fixed charges are fixed in number, then the number of ions going in will be determined by the number of fixed charges present. In this case you can write down an equation which formally is analogous to the Michaelis-Menton equation for enzyme action. More importantly, if you study two species of ions going in simultaneously, then you will find that the one will affect the other in the rate of going in and 
that if you plot the rate of entry of one ion at different concentrations in the presence of another, also at varying concentration, then they should exhibit the characteristics of the Michaelis-Menton equation for competitive inhibition. The results with many isotopes have borne out this postulate. In other words, ions do not simply go in by going through a uniform surface but seem to associate and disassociate with these charges.

With this in mind, I think I will just go back to the original argument that I would like to raise as to whether to call a phase like that liquid or not. If you have these highly charged particles situated on it, both positive and negative, there will be considerable tendency for these charges to repel and attract each other, and the situation, of course, is very similar to our macromolecules. It is rather difficult to think that their forces of cohesion are entirely dependent upon the van der Waals forces at the same time when they are acted on by much stronger forces between the molecules. In other words, I am not fundamentally differing with it, but I would say there might be possibilities that these cylindrical molecules as Dr. Mullins presented them in his picture might be joined by stronger bonds than the comparatively weaker ones.

Dr. Mullins: To answer Dr. Reynolds' question about the rare gases, especially xenon, since it is a good anesthetic at a partial pressure of about 500 millimeters of mercury, it is of course very small and it is subject only to dispersion forces for its binding, but that simply means that it will occupy a little niche or cranny somewhere around the surface and you may require two of them or three of them at a given site in order to produce block.

In the second case, I think that there is very good evidence that when you have distorted a site by some of these excitatory substances that I mentioned then large molecules which would be excitatory will now develop an inhibitory action. 


\title{
IV
}

\section{SUMMARY}

\author{
Robert G. Grenell
}

Psychiatric Institute, Medical School, University of Maryland, Baltimore, Md.

W

E HAVE ATTEMPTED in the short course of this symposium to cover several complex and fundamental areas of consideration at various levels of function and integration in the nervous system. To summarize all of this material, its implications and the questions to which it gives rise, would be impossible at this time. However, I am somewhat startled to realize how much information and suggestion for future experimentation are revealed by the material presented and discussed in this short day and a half.

In general, the problems being examined were those at the molecular and grosser biochemical levels which must be considered in an effort to understand the mechanisms - membranal, intraneuronal and interneuronal-whereby stimuli are received, interpreted and transformed into meaningful informationcarrying nervous messages which lead to the behavioral responses of the organism.

The first two papers dealt respectively with "chemical" and "physical" receptors. Dr. Dethier described experiments dealing with the concept of the general action of chemical stimuli. This type of investigation is at one end of the continuum from chemical source to electrical output. Dr. MacNichol, in discussing the electrophysiology of photoreceptors, showed how another type of approach can begin at the electrical output end and work back in the direction of the chemical. These approaches are brought closer together by consideration of, "how in a given receptor, the energy released by the initial mechanical, chemical or thermal event is amplified and converted into the kind of information carried by all nerve fibers." This is the "transduction" problem about which we are trying to assess what we know and what leads exist to what we do not know. Several points have been brought out here, however, which would appear to lead to a general hypothesis. It has been demonstrated that very small changes in molecular structure are reflected in pronounced physiological changes. Stereoisomers have, as Dr. Dethier pointed out, different tastes. It might be added that more recently it has become apparent that steric properties play a fundamental role in olfaction, as well as in the responses of cerebral motor neurons 
(e.g. production of convulsions) and above all in the total behavioral response of the organism. One example of this last effect is the difference in action of two of the new "psychiatric" drugs-Frenquel and Meratran. These two substances are isomers. Meratran, however, is a stimulant, while Frenquel is a tranquilizer and blocker of such drugs as LSD-25 (which produces hallucinations). It cannot be overlooked, therefore, that molecular arrangement is fundamentally related to nervous activity.

In addition, a second molecular problem has been brought up in these presensations of "reception." This is the problem of receptor sites-loci in the cell surface, the properties of which relate to the effectiveness of molecules to which they are exposed. The question, broadly, is concerned with the importance of molecular size, shape and other properties in relation to the fit of the molecule into a particular site (whether this be enzymatic or not). These questions are raised again later in the symposium particularly by Drs. Fischer and Mullins. It is clear that such properties as stimulus reception and response (including certain characteristics inherently part of what we call threshold) a re directly and fundamentally related to certain molecular characteristics and bonding. It has been proposed that the most crucial of these are molecular shape and cohesive energy density. The importance of and interrelationships between the problem of molecular shape and size and the concepts of receptor sites has been brought out in more detail by some of Dr. Mullins' considerations. He has attempted to set up a hypothesis (on the basis of both logic and experiment) which could serve to explain why certain substances stimulate and why others block, as well as to deal with the even greater complexity of the bimodal response of neural structures as the concentration of a particular substance is increased. Dr. Davies discussed some more classical concepts of membrane structure. Although these two presentations are quite different, it is of great interest and importance to future work that they can be fitted together in certain respects. Although both concepts ultimately become involved with "leakiness" of the membrane, and thus with changes in ion permeability, it is hard to see the mechanism in the more classical approach, which would deal with the question, for example, of the initiation and amplification of excitation. On the other hand, Dr. Mullins' views do offer something quite specific in this regard. It is of further importance that both these discussions and that of Dr. Fischer, which points out certain other considerations of "membrane" binding, have given us a much more advanced picture of a membrane which changes structure as physiological conditions change. As Dr. Ling pointed out, it is not impossible to fit his fixed-charge hypothesis into a membrane theory such as that suggested by Dr. Mullins. All of these views suggest further problems and experiments dealing with separation of membrane effects from intracellular non-membrane ones; the relationship of membrane effects to intracellular substrate production; the 
question of the membrane as an enzyme; the relationship of bonding, membrane effects and activity, etc., etc.

In general, it appears at this time (early in the development of such approaches to these problems) that reception-at least for the processes of olfaction and taste-is "more nearly biophysical than biochemical." The problem is not yet as clear in the area of photoreception. Here there is the initial photochemical process beyond which little is known from this point of view. It is important, however, as Dr. MacNichol stated, that "dark adaptation takes place in the electrochemical amplifying mechanisms activated by the splitting of the photo-pigment and resulting in the production of the graded slow potential." Basically, we are dealing here with a charged or trigger system which presumably involves biochemical concepts similar to those with which Dr. McElroy has been dealing in the problem of conversion of chemical energy into light energy and vice versa.

Such considerations lead us to chemical mechanisms of a grosser nature occurring within specialized cells. In certain respects there are both decided agreements and decided differences of opinion in this area. It appears that we are all agreed that the classical thoughts with regard to the roles of glucose and rates of oxygen consumption must be considerably changed. I might add, as an aside, that this is particularly gratifying to Dr. Davies and myself, when we remember the consternation with which we were met in 1950 when we first reported that glucose withdrawal did not affect the $Q \mathrm{O}_{2}$ of cerebral cortex-at least over a period of several hours. It is true, however, that lack of effect on rate of oxygen consumption cannot by itself be taken to be equivalent to absence of effects on metabolism. As Dr. McElroy has pointed out, and as our experimental results have shown, many other metabolic factors must be considered - substrate sources and types, electron transfer mechanisms and others. Dr. Larrabee's calculations can be questioned on the basis of the assumption of a $\mathrm{P}: \mathrm{O}$ ratio of 3 . I should hesitate to conclude from this information that either the energy level is not important or that intracellular chemical reaction systems have not been altered. With the state of our knowledge as it is at the present time there is no reason to believe that functional activity of neural units cannot derive from initial membrane changes associated with subsequent intracellular biochemical reactions which, perhaps through effects on high energy phosphate bond production, lead to reversible (or irreversible, in special cases) changes in cell structure and biosynthetic mechanisms. As Dr. Ling put it, ATP would somehow be important for the maintenance of electrical anatomy (transport mechanisms, for example).

In general, then, it remains for the future to close still further the physical and chemical gaps relating membrane structure, breakdown and resynthesis (this last being largely an intracellular biochemical job) with the various phases and properties of neuronal responses. 




